\title{
The development and function of illness scripts : studies on the structure of medical diagnostic knowledge
}

Citation for published version (APA):

Custers, E. J. F. M. (1995). The development and function of illness scripts : studies on the structure of medical diagnostic knowledge. [Doctoral Thesis, Maastricht University]. Datawyse / Universitaire Pers Maastricht. https://doi.org/10.26481/dis.19950601ec

Document status and date:

Published: 01/01/1995

DOI:

10.26481/dis.19950601ec

Document Version:

Publisher's PDF, also known as Version of record

\section{Please check the document version of this publication:}

- A submitted manuscript is the version of the article upon submission and before peer-review. There can be important differences between the submitted version and the official published version of record. People interested in the research are advised to contact the author for the final version of the publication, or visit the DOI to the publisher's website.

- The final author version and the galley proof are versions of the publication after peer review.

- The final published version features the final layout of the paper including the volume, issue and page numbers.

Link to publication

\footnotetext{
General rights rights.

- You may freely distribute the URL identifying the publication in the public portal. please follow below link for the End User Agreement:

www.umlib.nl/taverne-license

Take down policy

If you believe that this document breaches copyright please contact us at:

repository@maastrichtuniversity.nl

providing details and we will investigate your claim.
}

Copyright and moral rights for the publications made accessible in the public portal are retained by the authors and/or other copyright owners and it is a condition of accessing publications that users recognise and abide by the legal requirements associated with these

- Users may download and print one copy of any publication from the public portal for the purpose of private study or research.

- You may not further distribute the material or use it for any profit-making activity or commercial gain

If the publication is distributed under the terms of Article $25 \mathrm{fa}$ of the Dutch Copyright Act, indicated by the "Taverne" license above, 


\section{THE DEVELOPMENT AND FUNCTION OF ILINESS}

SCRIPTS

Studies on the structure of medical diagnostic knowledge 



\title{
THE DEVELOPMENT AND FUNCTION OF ILLNESS SCRIPTS
}

Studies on the structure of medical diagnostic knowledge

\author{
PROEFSCHRIFT
}

ter verkrijging van de graad van doctor aan de Rijksuniversiteit Limburg te Maastricht, op gezag van de Rector Magnificus, Prof. Mr. M. J. Cohen, volgens het besluit van het College van Dekanen, in het openbaar te verdedigen op donderdag 1 juni 1995 om 16.00 uur

door

Eugène Jean François Marie Custers

geboren te Swalmen

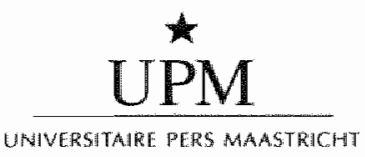




\section{PROMOTOR}

Prof. dr. H. G. Schmidt

\section{CO-PROMOTOR}

Dr. H. P. A. Boshuizen

\section{BEOORDELINGSCOMMISSIE}

Prof. dr. H. F. M. Crombag (voorzitter)

Dr. P. J. Braspenning

Prof. $d r . J . J$. Elshout (Universiteit van Amsterdam)

Prof. dr. J. Jolles

Prof. dr. V. L. Patel (McGill University, Montreal, Canada)

Produktie: Datawyse | Universitaire Pers Maastricht

CIP-DATA KONINKLIJKE BIBLIOTHEEK, DEN HAAG

Custer, Eugène Jean François Marie

The development and function of illness scripts : studies on the structure of medical diagnostic knowledge /

Eugène Jean François Marie Custers. - Maastricht :

Universitaire Pers Maastricht. - III.

Thesis Rijksuniversiteit Limburg Maastricht. - With ref. With summary in Dutch

ISBN 90-5278-187-7

Subject headings: medical diagnostics / medical expertise / cognitive psychology. 


\section{CONTENTS}

Chapter 1. Historical and current perspectives on medical expertise 1

Introduction 1

Problem solving: General overview 3

Knowledge representation: General overview

Production systems and connectionist architectures 8

The representation of concepts and categories 8

Schemas 11

Scripts 13

Expertise research: General overview 16

Studies in medical expertise $\quad 21$

General framework of the present studies: The illness script concept $\quad 28$

Evidence for the cognitive reality of illness scripts 31

Overview of the present studies 33

Chapter 2. The development of illness scripts: Results of a "scrambled case" recognition study

Introduction $\quad 35$

Method 37

Results $\quad 39$

Discussion $\quad 42$

Chapter 3. The Relationship between medical expertise and the development of illness scripts: Evidence from an interview study

Introduction 45

Method 49

Results $\quad 52$

Number of statements in different illness script categories $\quad 52$

Proportion of statements in different illness script categories $\quad 56$

Relationship between actual clinical experience with the diseases and quality of the descriptions $\quad 59$

Discussion

Chapter 4. The influence of medical expertise and case typicality on case processing

Introduction

Method 72

Results $\quad 76$

Case reading times $\quad 76$

Case evaluation latencies (decision times) $\quad 80$

Discussion 
Chapter 5. A recognition study testing the psychological validity of illness scripts

Introduction $\quad 89$

Method $\quad 97$

$\begin{array}{lr}\text { Results } & 102\end{array}$

Recognition memory scores $\quad 102$

Recognition reaction times 108

Reaction times for different types of recognition responses: Comparison of the present results with the Yekovich \& Walker (1986) data

Discussion

$\begin{array}{ll}\text { Chapter 6. Summary of the results and general discussion } & 121\end{array}$

Introduction $\quad 121$

Summary of the present results 121

Implications for the representation of medical knowledge and medical problem solving

The development of medical knowledge $\quad 129$

Implications for medical education 131

Suggestions for further research 133

Samenvatting (Summary in Dutch)

137

References

143

Appendices

155

Acknowledgements 


\section{CHAPTER 1}

\section{HISTORICAL AND CURRENT PERSPECTIVES ON MEDICAL. EXPERTISE}

\section{INTRODUCTION}

It is amazing how long psychology has resisted the temptation to study the nature and development of expertise, particularly because the reluctance to engage in other cognitively complex domains has been much smaller. For example, many important studies in fields like cognitive development (e.g., Piaget, 1929), consciousness (e.g., Prince, 1921), problem solving (e.g., Maier, 1930, 1931; Selz; 1922), and memory (cf. Bartlett, 1932/1954; Semon, in Schacter, 1982), figured already prominently in standard introductory works before psychologists even started to formulate opinions about the nature of expertise. In addition, the lack of an appropriate theoretical framework can not count as an explanation for the virtual absence of expertise studies until late in the 1960s, for de Groot's (1946/1978) study on thinking and expertise in chess remained for decades almost whout succession. The current cascade of expertise studies largely springs from the field of artificial intelligence (e.g., Feigenbaum \& Feldman, 1963) and from efforts by people who work in a particular area to model expertise in their own subject-matter domain (e.g., Wortman, 1966), rather than from a genuine interest from the part of cognitive psychology.

Actually, amateur "expertise research" has a much longer history than its scientific counterpart. Socrates (469-399 B.C.), one of the founding fathers of western philosophy and science, was already bothered with the issue of what makes an expert an expert (Dreyfus \& Dreyfus, 1984). Eutyphro, prophet and contemporary of Socrates, was a self-acclaimed expert in the domain of piety. Applying his own experimental method, the "Socratic dialogue," to this subject, Socrates tried to find out by what rules and standards Eutyphro judged a person to be pious. However, the prophet was unable to provide these rules and standards; instead, he presented examples of people and gods who had, in his opinion, display. ed pious behavior in certain circumstances. Socrates, being familiar with these examples, was not impressed; hence, he did not further pursue these first attempts to study the nature of expertise.

Was Socrates the first one to conduct an empirical study on the nature of expertise, Socrates" spokesman Plato (428/427-348/347 B.C.) receives credit for being the first to develop a theory about expertise: he conjectured that experts originally have known the rules and standards by which they judge and act, but that they have forgotten them in the course of time. According to Plato, philosophers should be assigned the task of rediscovering these rules. Surprisingly, this is precisely what much of the current expertise research, though no longer the exclusive domain of philosophy, is still about. Laypeople tend to describe expertise in terms of talent, knowledge, experience, and skills (cf. Boshuizen, 1989). The question whether experts use rules, and if they do, what the nature of these rules may be, is still not settled some 24 centuries after Socrates' and Plato's first explorations (e.g., Dreyfus \& Dreyfus, 1986b). In fact, for most of the intervening years, research on the nature of expertise did not feature on the agenda at all. 
Some reasons why questions about the nature of expertise regained scientific attention a few decades ago were already mentioned: the development of expert systems and increasing interest from within the different fields. On the other hand, it is also safe to notice that, to a large extent, psychology was "ready" to study the nature of expertise: Progression on a number of research topics in psychology and cognitive science had paved the way for the investigation of complex human behavior. Research in several fields has contributed to this achievement, including studies in knowledge representation, human thinking, problem solving, reasoning, decision theory, categorization and classification, and text processing. Soon after scientists began to understand these complex human behaviors, the topic of human expertise in several domains emerged as a particularly interesting domain to apply these newly acquired insights to. From a scientific point of view, experts are valuable subjects, if only because they constitute the ultimate test for psychological theories of learning and expertise development. From a practical point of view, it seemed likely that much was to be gained: to become an expert in a domain generally takes a person several years and a society corresponding investments; even relatively minor accelerations and improvements in the training of experts could produce large savings.

Two subdomains of cognitive psychology have been particularly influential in the development of theories and the design of experiments concerning the nature of human expertise: problem solving and knowledge representation. Though studies on problem solving are relatively process oriented and tend to emphasize observable behavior, while studies on knowledge representation are more focused on formal descriptions of complex knowledge structures, especially in recent years a considerable number of studies have been performed that can be subsumed under both headings. For example, in almost all research on problem solving by analogy (e.g., Anderson \& Thompson, 1989; Gick \& Holyoak, 1983), issues of problem solving and knowledge representation are jointly addressed. In a similar vein, theories on expertise are faced with intricate relationships between problem solving and knowledge representation.

In the following sections of this chapter, the implications of research on problem solving and knowledge representation for current conceptualizations of expertise in general, and expertise in the medical professions in particular, will be discussed. Expertise studies that are not rooted in either of these research strands will only be discussed indirectly ${ }^{1}$. We will start with a concise overwiew of theories and research on problem solving and knowledge representation. Next, a section will be dedicated to expertise research in a variety of domains, followed by a more elaborate section on the relevant studies in the medical domain. Finally, we will explain our conceptualization of what we see as the core of medical diagnostic expertise, the illness script theory, and subsequently sketch the experiments performed to investigate whether this concept is in fact viable.

1 For example, studies conceming the physical or psycho-motor skills involved in expert behavior, will barely be touched upon. Similarly, we will not delve deeply into studies that anm mainly at investigating whether expert behavior is in line with certain prescribed professional or ethical standards. Though it surely can not be denied that these aspects of expertise are interesting and important, we think that they fall largely outside the scope of cognitive psychology. 


\section{PROBLEM SOLVING: GENERAL OVERVIEW}

Ever since the beginning of modern psychology in the second half of the 19 th century, psychologists are troubling their subjects with problems and tasks. As such, problem solving research probably is as old as psychology itself. Early relevant work was performed in William Wundt's psychological laboratory in Leipzig, with its official start in 1879. Wundt and his co-workers tried to trace the process of problem solving by requiring subjects to perform introspection: They had to report on their experiences during problem solving, either on-line or post-hoc. However, growing scepticism about the feasibility of studying the contents of human consciousness ultimately culminated in the behavioristic approach dominating psychology from about 1910 until 1955, particularly in America and the United Kingdom. Behaviorism did not encourage psychologist to engage in research on problem solving, except for its simplest forms under direct control of environmental reinforcements. Meanwhile, in Europe, Gestalt psychologists like Wertheimer, Köhler, and Maier, remained interested in the topic, especially in insight problem solving. As this type of problem solving generally involves mainly perceptual problems, the product, rather than the process of problem solving was emphasized: Insight problems do not lend themselves particularlly well to the study of problem solving processes. A notable exception in this respect was Otto Selz (1920), at that time the only scientist who developed a theory of problem solving with an emphasis on thinking and problem solving operations (cf. de Groot, 1946/1978).

By the mid-1950s it became clear that behaviorism was unable to deal with the intricacies of human behavior, and that mental operations had to be postulated in order to be eventually able to account for human problem solving. In the next two decades, approximately from 1955 to 1975, problem-solving literature focused mainly on these mental operations that were assumed to operate between stimulus and response (cf. VanLehn, 1989). The main objective of most researchers during these years was to find and describe a small set of generally applicable mental stages that could mediate between potentially any stimulus and response (cf: Miller, Galanter, \& Pribram, 1960). Stimulus and response itself were kept relatively simple and well-defined; emphasis was laid on the mental processes applied to transform the stimulus into the response. This type of research culminated in the monumental. Newell and Simon (1972) volume on human problem solving.

Especially salient in this view was the distinction between ill-defined problems and well-defined problems. Initially, problems were considered welldefined if a test existed, easily performable by the problem-solver or problemsolving system, on basis of which it could be determined whether an object proposed as a solution, indeed was a solution (Newell \& Simon, 1972, p. 73). I1ldefined problems lack this feature. However, it is probably more profitable to conceive the distinction well-defined versus ill-defined as a continuum. On the one end of this continuum, there are well-defined problems, characterized by a problem description that contains, implicitly or explicitly, all the information necessary to solve the problem, and an unequivocally testable solution. A very limited number of operators is available that can be used to successively transform the initial problem situation into a solution. These operators can best be described as the application of elementary rules in a certain domain, like modus tollens and modus ponens in logic, the use of distributive and associative properties in arithmetic, or addition and multiplication in calculation. In addition, more domainindependent operations like insertion, assignation (of a value to a variable) and 
substitution (of one symbol for another) can also function as operators. In games like chess, checkers, and tic-tac-toe, the operators are the legal moves defined by the rules of the game.

At the opposite end of the continuum, ill-defined problems, like "how to become a successful artist in one year, ${ }^{\text {, }}$ or "how to decrease the national budget deficit without rendering too many people unemployed" can be found. For these problems, neither the problem space, nor the set of available operators can be well-defined; in fact, both can only be approximately described and are often apparently dependent on the vantage point from which the problem (space) is viewed. Furthermore, no solution can be unequivocally tested (e.g.s. When should an artist be considered "successful"?). However, many problems are situated somewhere half-way on the continuum of well-defined versus ill-defined. For example, though there is a generally agreed-upon correct solution to insight problems, like the frequently used Duncker's radiation problem ${ }^{2}$, it is difficult to exactly define operators that are applicable in the problem space. Similarly, in medicine, the domain to be discussed in this thesis, problems can also be classified on this well-defined versus ill-defined continuum. For example, it seems reasonable to assume that diagnosis of an infectious disease is a relatively welldefined problem, whereas the question how to improve the quality of life of seriously ill people often will be relatively ill-defined.

Researchers tend to prefer well-defined problems over ill-defined problems as experimental tools, because solving a well-defined problem can be described as applying a sequence of operators to transform the initial problem state through a number of intermediate problem states into the goal state or solution. Thus, the problem solving process of well-defined problems can be completely traced, step by step, while this is often impossible for ill-defined problems. For example, insight problems are often solved by a sudden transition ("flash of insight") from the initial problem state to the, often remote, goal state; it is difficult to isolate the application of individual operators from this insight process.

Well-defined problems are usually solved by applying problem solving methods. A method can be conceived of as a pattern or chain of successive stages of operation selection, state evaluation, and goal creation. In fact, the elaborate description, classification and analysis of problem solving methods provided by Newell and Simon (1972) is probably the primary reason why this work has been so influential. Problem solving methods that guarantee an appropriate solution are called algorithms, problem solving methods that do not infallibly yield such a solution are called heuristics. Newell and Simon (1972) define a number of heuristics that they consider generally applicable to a wide range of to problems. Examples of these heuristics are means-ends-analysis, forward search, backward search, and breadth-first search.

Nevertheless, in contrast to their claim of generality, the range of problems Newell and Simon base their theory on is actually limited to a very particular type of tasks: cryptarithmetic problems, theorem proving in logic, chess, and some specific puzzles like tic-tac-toe. The most salient aspect of these problems is that they are not only well-defined, but also that they originate from closed, highly

2 In short this problem boills down to the following situation: A doctor is faced with a patient who has a malignant tumor in his stomach. This tumor can only be destroyed by radiotherapy. However, if rays are used with an intensity sufficient to destroy the tumor, the healthy tissue that the rays have to pass on their way to the tumor will also be destroyed. Is there a way to destroy the tumor with rays of sufficient intensity, while at the same time avoiding destruction of the healthy tissue? For the details about this problem and its solution, see Gick \& Holyoak (1983). 
formal domains, with all the information necessary to solve the problem in principle avalable from the axioms and rules of the domain. Problem solvers do not need to bring to bear information from outside the formal domain to solve these problems. However, consider the following problem:

Which inventor sold only 200 copies of his philosophical work Solidarismus ${ }^{3}$

Example 1.1. Instance of a knowledge-rich problem

This problem, though being well-defined according to the Newell and Simon (1972) definition, is difficult to solve if one does not know the answer immediately. Similarly, Duncker's radiation problem can hardly be solved by subjects who are ignorant of the properties of lenses. It is not possible to derive, in a formal way, the properties of lenses from knowledge about tumors or radiation, let alone the personal particulars of a specific inventor from the sales figures of a philosophical work. Problems that can be solved by making use of the formal rules provided by the underlying domain (e.g., logic, chess, mathematics), and that do not require external semantic or "real world" knowledge, are called knowledge-lean problems; problems that do require this latter type of knowledge in order to be solved, are called knowledge-rich problems. Knowledge-lean problems have been (and still are) particularly popular among researchers trying to discover and model the more formal properties of human problem solving, for example, in artificial intelligence. Research employing knowledge-rich problems, on the other hand, often is motivated by an intrinsic interest in the content of the knowledge necessary to solve these problems.

As long as knowledge-rich problems can be solved by simply retrieving an information item from memory, like the "inventor problem" in Example 11.1 , their usefulness for scientific study is likely to be limited. The situation becomes more interesting when larger amounts of knowledge of a particular field are involved in problem solving. Real-world problems in, for instance, economics, geography, physics, history, medicine, law, or politics usually require extensive amounts of relevant domain knowledge to be solved. In fact, researchers like Newell and Simon, who tend to focus on general, domain independent problem solving methods, do not deny that domain knowledge is important, even for the relatively formal and knowledge-lean problems they use as experimental materials (cf. Newell \& Simon, 1972, p 82, 790). However, what they do is drawing a sharp line between problem solving methods on the one hand, and knowledge of the problem domain on the other hand. The way they describe the use of knowledge in problem solving highly suggests that, in their view, knowledge should be conceived of as a data base filled with individual facts, upon which operations, like storage and retrieval, can be performed. In order to account for the possible influence of the quality of this knowledge, they invoke the concept of intelligence. They discuss an example of a less intelligent information processing system crossing a chasm by going over a bridge, while a more intelligent information processing system would invent the airplane to overcome such a gap in the landscape (Newell \& Simon, 1972, p. 82). Evidently, the "intelligence" of such a system able to invent an airplane implies the presence of large amounts of knowledge in a number of domains. The idea that knowledge, intelligence, and problem

3 For the solution of this problem, the reader is referted to the end of this chapter 
solving abilities are unrelated entities has been gradually abandoned in the last two decades. For example, the hierarchical, network-like description of the declarative memory component of $\mathrm{ACT}^{*}$ (Anderson, 1983) can be seen as an attempt to impose structure upon the knowledge base, while still preserving the underlying general processing mechanisms, i.c., the production system architecture. In recent psychological views, domain knowledge, and especially the way it is structured, is seen as intricately related to problem solving capacities in that particular domain. Domain-specific problem solving heuristics are powerful and require an extensive amount of knowledge of the domain they are applicable to. On the other hand, the generall, i,e, domain independent, problem solving heuristics, elaborated upon by Newell and Simon (1972), are usually avoided by human problem solvers, at least if they are able to substitute domain knowledge and domain specific heuristics for these. Indeed, it was in the very domain of chess, a game with well-defined and highly formal rules, and hence, especially amenable to the Newell and Simon (1972) approach, that one of the earliest studies appeared in which the structure of domain knowledge was emphasized (De Groot, 1946/1978). De Groot's view that the structure of chess knowledge determines, to a large extent, the quality of chess thinking and performance, ultimately radiated to almost all problem solving domains; in fact, problem solwing nowadays can be considered almost equivalent to knowledge application (cf. Boshuizen, 1989). The rather disappointing results from general problem solving systems in artificial intelligence, at least as far as the application of such systems to semantically rich domains is concerned, have also contributed to this new approach. Presently, it remains an open question whether a production system architecture, like the one proposed by Anderson (1983) can completely account for the effects of the structure of knowledge on problem solving.

The role of learning by solving problems is also an issue that is relatively underrepresented in the work of Newell and Simon (1972). As might be expected, learning can take place by adding elements to the problem space, the current knowledge state, or a specific problem solving program or algorithm. According to Newell and Simon, these new elements are cast in the form of productions; it may be assumed that, if these are subsequently stored in LTM, the system has learned something. However, Newell and Simon are not explicit about the underlying mechanisms of learning. Anzai and Simon (1979) emplay the notion of an adaptive production system, able to learn from both its successes and failures, to incorporate learning mechanisms. According to Newell (1990), all forms of learning are mediated by chunking of productions. The problem with these approaches is that problem solving is still conceptualized as something that can be learned independently from problem content. For example, Anzai and Simon (1979) base their general theory on the thinking-aloud protocol of one subject solving one type of knowledge-lean tasks, i.c., the Tower of Hanoi puzzle. However, as already mentioned, in more recent research, problem solving is approached as a function of domain knowledge application; hence, learning to solve problems is viewed as development of knowledge, or, in a broader sense, as development of expertise in a particular domain. The idea that people, or, for that matter, systems, can be good problem solvers in general (one would almost be tempted to say experts in the domain of problem solving), has gradually lost much of its attractiveness, to be replaced by studies that focus on knowledge development, knowledge application, and problem specificity.

Probably, still the most outstanding study on problem solving in medicine is the work of Elstein, Shulman, and Sprafka (1978), though the influence of this work extends well beyond the confines of the field of medicine. In line with the 
spirit of the 1970s, the aim of the researchers was to discover formal problemsolving features to distinguish excellent from non-excellent physicians. It was investigated whether medical problem solving performance could be predicted by variables like amount of information sought, number of diagnostic hypotheses ventured, or time lapsed before the first hypothesis was formulated. However, most of the problem-solving characteristics investigated in this study failed to yield any significant differences between outstanding and average physicians. The fact that from the late 1970 s onwards, efforts to discriminate on basis of formal problem solving features between subjects differing in medical proficiency have almost completely vanished from the stage, underscores the soundness and the impact of the Elstein et al. (1978) work.

\section{KNOWLEDGE REPRESENTATION: GENERAL OVERVIEW}

From the discussion in the previous section it may be concluded that medical diagnostic problems, as a subclass of knowledge-rich problems, can only be solved if the relevant knowledge is available; general problem solving methods, like those described by Newell and Simon (1972), are of limited use. However, though in many situations this domain knowledge may be assumed for even demonstrated) to be present; in actual situations, it often can not be retrieved by a subject faced with a problem. In general, such retrieval failure is a consequence of the proper knowledge not being activated by reading or hearing the problem (cf. Anderson, 1983). Whether or not knowledge is activated and used in a particular situation is dependent on the features of both the knowledge itself and the problem solving situation; presently, we will be concerned mostly with features of the knowledge itself, hence, with the way the knowledge is represented in the subject's memory.

Unfortunately, cognitive psychology is faced with the situation that there are many, often functionally equivalent, ways to describe knowledge representation. Researchers may select the representational format or combination of formats that optimally suits the particular type of knowledge they are investigating; models with highly similar explanatory power may be spuriously contrasted. Some of the options are: rule-based representations (Bruner, Goodnow, Austin, 1956; Smith, Langston, \& Nisbett, 1992), representations based on similaritios, instances, or exemplars (Read, 1983; Ross, 1987; Ross, Perkins, \& Tenpenny, 1990), prototypes (Rosch, 1978), individual cases (Kolodner \& Riesbeck, 1986), (lay) theories (Medin \& Ortony, 1989; Murphy \& Medin, 1985; Wisniewsky Medin, 1994), mental models (Gentner \& Stevens, 1983; Johnson-Laird, 1983), frames (Minsky, 1975), schemas (Bartlett, 1932/1954), and scripts (Schank \& Abelson, 1977). These functional knowledge representation models are often embedded at a more structural level in a production system architecture (cf. Anderson, 1983), a connectionistic network (cf. McClelland \& Rumelhart, 1986), or a hybrid or mod ular structure (cf. Fodor, 1983; Holyoak, 1991; Schyns, 1991).

In the next sections, we will briefly review some of these theories of knowledge representation, as far as they are relevant for current approaches of expert knowledge representation in general and medical expertise in particular. 


\section{Production systems and connectionist architectures}

At the lowest level, knowledge is represented by neurons and neural activity in memory. Presently, the ability to account for cognitive processes by describing neural processes is limited; there is considerable debate about the possibility of reducing psychological activity to neural activity, or about the extent that the "software" is independent from the "hardware." Models like ACT* (Anderson, 1983) and SOAR (Newell, 1990) assume that human cognition can be described completely as symboll manipulation; consequently, hardware and software are independent, and expertise can be programmed independent from the underlying physical device (e.g., the brain, or a computer). In models based on neural networks, on the other hand, knowledge is represented as patterns of activation in a network of units (Bechtel \& Abrahamsen, 1991); the assumption here is that any form of cognition, including expertise, is an inherent consequence of such activation patterns, and hence can not be described apart from the functional properties of the underlying network. For example, according to these latter models, the rules an expert apparently adheres to are epiphenomena; knowledge representation actually amounts to patterns of activation in a network of nodes or units. Therefore, proponents of a connectionist view of cognition are often reluctant to invoke concepts at a psychological level of explanation, like rules, models, schemas or scripts, to account for human cognition. In their view, any kind of cognitive behavior can be sufficiently explained by the properties of the human brain.

It has been demonstrated that connectionist models are able to learn concepts and to categorize instances of these concepts as a result of repeated presentation of exemplars of different concepts in combination with simple feedback (e.g., Schyns, 1991). However, the possibility of such systems learning the complex concepts employed by human experts, without the insertion of an intermediate psychological level, like the simple operations in SOAR, is still remote.

Most current expert systems are based on a production system architecture, with the expert knowledge represented by a large number of condition-action rules. These systems often fare relatively well, as long as their domain of application is limited. An important drawback of these systems is that if the system fails to find a match between the current input and any of the conditions, performance often drops to zero, while human experts are often able to come up with acceptable sub-optimal solutions. Presently, the notion that all human knowledge can be represented by a production system architecture, is still contested. In recent years, connectionist and production system architectures are developing towards each other (Bechtel \& Abrahamsen, 1991), and "hybrid" models are being constructed (cf. Holyoak, 1991).

\section{The representation of concepts and categories}

According to Lakoff (1987), concept learning and categorization are the most basic aspects of human thinking, perceiving, acting, and speaking; as such, they also play a crucial role in human expertise. For example, medical diagnostic expertise, or at least routine diagnostic problem solving, can be viewed to a large extent as categorization behavior, with the diagnoses playing the role of categories (Norman, Rosenthal, Brooks, \& Muzzin, 1989; Weber, Böckenholt, Hilton, \& Wallace, 1993; Wortman, 1970). Categorization is especially important for prediction; for example, if someone is categorized as "having the flu," certain predic- 
tions about the symptoms he shows, his behavior in the near future, and the course of action to be taken by the physician can be made. Indeed, the fact that people consulting a physician often insist that a diagnosis should be voiced (and, consequently, are disappointed if the doctor is unable to do so), underscores the importance of the categorization aspect of medical diagnosis.

Early studies in categorization, employing simple geometric forms like circles, triangles and squares, and mostly designed to measure the learning of simple, but artificial constructs like logical rules, date back to the $1950 \mathrm{~s}$ (Bruner, Goodnow, \& Austin, 1956).

In the classical view of concepts and categories, features or properties that define a concept are individually necessary and jointly sufficient to determine category membership (Medin, 1989). The logical concepts used by Bruner et al. (1956) conform to this view. Category membership of individual instances can be unambiguously determined. A corresponding approach in medicine would be to describe diseases and categorize patients on basis of defining or pathognomonic symptoms. Some authors indeed describe the clinical features of diseases in a way that implicitly is in keeping with such a view (e.g., Wortman, 1966, 1970). However, the situation in medical diagnosis is seldom as clear-cut as in Bruner' $\mathrm{g}$ task, and unequivocal classification of a case on basis of defining signs and symptoms is an exception rather than a rule.

Most real-life concepts, including conceptualizations of almost all diseases, are impossible to describe by defining features; category representativeness is often a continuum, and some category members are better examples of a cacegory than others. The classical example is that of the robin being a better example of the category "bird" than the penguin, though both are, by definition, birds. For many categories, defining features are even completely lacking; for example, in the case of membership of the category "means of conveyance." Little imagination is required to observe that a Zeppelin, a car, and a camel are difficult to describe by common defining features; nevertheless, all three are means of conveyance. To deal with this type of categories, a number of alternatives have been devised for the classical view; these alternatives can be subsumed in three groups:

1) Probabilistic theories, in which category membership is not an all-or-none affair, but probabilistically determined by the sum of weighted features or the computed distance, in a hypothesized space, to a prototypical category mem. ber. Probabilistic theories include family resemblance models, prototype mod. els, and additive (or independent) cue models (Smith Medin, 1981).

2) Exemplar theories, i.e., theories that state that concepts are represented by previously seen instances, and that the representations of these individual instances are used to categorize new instances (Norman, Brooks, Allen, \& Rosenthal, 1988).

3) Psychological essentialism (Medin \& Ortony, 1989), which views concepts and categories as context dependent, organized around intuitive theories (Murphy \& Medin, 1985), or goal derived (Lakoff, 1987; Medin, 1989). Psychological essentialism aims at explaining, for example, why people are able to construct categories like "means of conveyance," "diet-food," and "things to save when your house is on fire."

Most relevant for routine medical diagnosis is the work in which prototype representations and instance-based representations are contrasted. Both types of theo ries assume that categorization is similarity based; the main difference between the two views is that prototype theories assume that an abstraction process takes place during category construction, whereas exemplar theories state that cat- 
egory membership of a new exemplar is determined by a "chorus" of individually represented instances.

In medicine, some researchers propose that disease concepts are represented around a prototypicality structure (Bordage, 1984; Bordage \& Zacks, 1984). For example, Bordage and Zacks (1984) found that physicians, novices and experts alike, in a free-recall paradigm, generate typical diseases for a certain category much easier and faster than atypical diseases. In addition, typical diseases were categorized much faster and more accurate than less typical diseases. However, Genero and Cantor (1987) found that the structure of psychiatric disease categories probably reflects the wy the categories are learned: medical experts who learned a disease on an exemplar base showed categorization behavior consistent with and exemplar model, while experts who learned a disease on basis of a summary prototype, showed categorization behavior consistent with a prototype model.

Proponents of instance based categorization models can also be found in medicine. For example, Norman et al. (1988) found that expert dermatologists made considerably fewer errors in diagnosing pictures of dermatological abnormalities than interns, but the proportion of misdiagnoses committed on prototypical instances of these abnormalities, compared to that for atypical instances, did not substantially differ between experienced and less experienced subjects: Experts still failed occasionally on highly typical cases. This finding is difficult to explain if diseases are represented as prototypes, because it may be assumed that experts have learned these prototypes thoroughly enough to be able to recognize them immediately.

Both prototype models and instance based or exemplar models assume that the structure of concepts and categories is similarity based, with a specific emphasis on perceptual aspects of similarity. Neither of these categorization models makes any assumptions as to why particular features co-occur; thus, they both suffer from a lack of constraints: anything can count as a feature (Smith \& Medin, 1981). This is a reason why it is often difficult to integrate category representation theories with other theories about knowledge representation, like semantic networks. If applied to diseases and medical diagnoses, both theories would hold that categorization depends on either learning the prototypes or on a number of experiences with actual patients. However, in diseases, clinical features are not arbitrarily associated with a disease; an underlying medical pathophysiological model exists that explains, in many cases, why certain features generally co-occur. In general, the issue of the theoretical foundation of medical diagnostic categories is closely related to the role of biomedical knowledge in diagnosis; we will re-address this topic in the forthcoming sections.

Most categorization research does not explicitly address the issue of category development or changes in category representation with increasing experience. There is evidence that experts in some domains have quantitatively more and qualitatively more refined categories than novices (Tanaka \& Taylor, 1991), though Murphy and Wright (1984) found that for three common pediatric disorders, experts have less distinctive representations than relative novices. This discrepancy might be related to the nature of the categories used in both experiments (birds and dogs in the Tanaka \& Taylor study, pediatric disorders in the Murphy \&. Wright study). However, the Murphy and Wright (1984) findings are difficult to reconcile with the Murphy and Brownell (1985) findings that more differentiated categories are easier to access, and that experts have very differentiated, easily accessible categories. The fact that category representations often change with increasing expertise is sometimes addressed by categorization theo- 
ries in terms of learning (e.g., Genero \& Cantor, 1987): Implicitly, many investigators seem to adhere to what Carey (1985) calls the "weak restructuring of knowledge" view: categories grow and become more refined, but do not fundamentally change.

In summary, though diagnosing routine cases might show a close resemblance to categorization behavior (Weber et al., 1993), diseases are more complex entities than the relatively simple utensils, like cups and bowls (e.g., Labov, 1973) often used in categorization research. In diseases, the features that determine prototypicality or atypicality are in a complex way interrelated. Furthermore, course is an important underlying variable in diseases, but not in categories. Consequently, in order to describe disease knowledge, we opt for a form of representation that is more able to handle these complex knowledge structures than categorization models.

\section{Schemas}

According to Bartlett (1932/1954) the first one to use the word schema in its present meaning, or something closely akin, was the neurologist. Henry Head (1920). Head used the word "schema" to refer to the hypothesized entities that "modify the impressions produced by incoming sensory impulses in such a way that the final sensations of position or of locality rise into consciousness charged with a relation to something that has gone before." Thus, Head's schemas play an important role in integrating incoming information and prior knowledge or experience. The words position and locality indicate that the schema has a "spatial" flavor.

About the same time, Otto Selz (1922), a German psychologist of the Würzburger Schule, introduced the idea of schematic anticipation (cf. de Groot, 1978). This notion is very close to what we now refer to as the problem representation: a schema is a sketchy anticipation of the attained goal (or problem solution), including the relevant information available in the data. The representation is necessarily sketchy, i.e., includes a gap, because the solution itself is not yet included in the schema; otherwise, there would not be a problem. The schematic anticipation is the starting point for the further course of the thought process. According to Selz, this thought process is controlled by general linkings between mental states, or between a problem situation and a mental state. Such linkings can be learned and subsequently evolve into "fixed thought habits." In this manner, Selz elaborated upon the classical concept of association. Selz's schematic anticipations are more reminiscent of physical schemas or even mental models than later conceptualizations of the schema idea, which seem to bear a closer resemblance to the fixed thought habits.

Bartlett (1932/1954, p. 45) introduces his concept of schema as follows: "It is now more obvious than ever that processes which are psychologically of very great complexity may appear, when they occur, to be of the utmost simplicity. This is because they are based upon the functioning of patterns which, once they are formed, operate as units; but their information itself may be a highly complicated matter." Bartlett's schema is to a large extent reminiscent of what would now be called a "motoric response pattern"; it refers to "an active organization of past reactions, or of past experiences, which must always be supposed to be operating in any well-adapted organic response" (Bartlett, 1932/1954, p. 201). However, and more importantly, the schema also has a semantic aspect: "A schema gives meaning to immediate perceptual data" (ibid., p. 45). The irony of psycho- 
logical history makes that Bartlett probably will live forever as the "inventor" of the schema, whereas he himself particularly disliked the word, finding it "at once too definite and too sketchy" (Bartlett, 1932/1954, p. 200-201).

A psychologicall theory like behaviorism, in which human behavior is viewed as complexes of simple stimulus-response associations, can completely dispense with the construct of a schema; therefore, the concept was in disuse for some decades, and Bartlett remained largely without successors. With the ascent of cognitive psychology from about 1956 onward, the concept of a schema regained in popularity. Generally, a schema in its present sense can be defined as a generic, higher-level mental structure that underlies the molar aspects of human knowledge and skill (cf. Brewer, 1987; Evans, 1989; Glaser, 1986; Rumelhart \& Norman, 1981, 1985; Turner, 1988; VanLehn, 1989; Wolters, 1983). Some authors emphasize the use of schemas in solving complex problems (Gick \& Holyoak, 1983; Holyoak \& Thagard, 1989; Koedinger \& Anderson, 1990; Sweller, Chandler, Tierney, \& Cooper, 1992). The idea of a schema in its present conceptualization is rooted in representational models in which the human mind is basically viewed as a semantic network consisting of nodes and relations between these nodes; in this approach, schemas are postulated higher-order representational units, providing additional structure in a semantic network (cf. Rumelhart \& Norman, 1985).

For several reasons, schemas are better tailored to represent medical diagnostic knowledge than the category representations described in the preceding section. First, schemas may be supported by model knowledge, and thus provide explanations why particular features co-occur, or why certain variables have correlated values in actual instances of the schema. Second, schemas are more complex structures, which contain not only information about typicality of separate features, but also information about covariation between features and about criticality of features for the schema (cf. Lesgold, 1984; Nisbett \& Ross, 1980); these latter aspects are not included in prototype based category representations. Instance based models cannot explain why two patients who resemble each other quite closely, but differ in some critical features, will be easily recognized as belonging to different disease classes. In addition, to develop accurate representations, instance based models would require many more instances actually presented than schema based models; schemas might be learned on basis of wery few instances. Third, schemas are better able to handle non-visual features than either instance-based or prototype based representations.

On the other hand, the notion of a schema has often been criticized as being too general (cf. Rumellhart \& Norman, 1985), and unable to do full justice to the particularities of many domains of real-world knowledge, including disease knowledge. What is especially lacking is a time course variable. Diseases, like many real-world events and activities, have an underlying time-based structure; also, in the diagnostic process, much of the information becomes sequentially available. Most conceptualizations of schemas do not include this aspect. Furthermore, knowledge of base rates (frequency of occurrence) and of seriousness of a disease are important variables that should influence the accessibility and activation of a particular schema (cf. Weber et al., 1993). Every physician is familiar with the concept of "diagnoses you'd preferably not miss," and this knowledge should also be incorporated into the schematic structure. Moreover, a diagnosis also includes implications for subsequent actions to be undertaken by the physician (e.g., prescribe medicine, refer to specialist), while a schema is more selfcontained. Hence, clinical disease knowledge might be better described by a specific type of schema, in case a script. 


\section{Scripts}

Though he did not use the word, a script-like idea was already expressed by Prince (1914/1921). In one of his lectures on memory, he states that "in a general way, events as they are successively experienced become associated together, and with other elements of personality, so that the latter recollection of one event in the chain of an epoch recalls successively the other." This notion of a sequence of interconnected events forms the core of the script concept. Particularly in two domains, the need for representations even more complex than schemas was urgently felt: in artificial intelligence, and in text processing. And though the idea of the script as representational structure originates from the former field, script related research has reached its full bloom in the latter domain.

In 1977, Schank and Abelson published their influential work "Scripts, plans, goals, and understanding," in which they unfolded a theory about the understanding of common human activities. An important starting-point was that form and content of knowledge should not be separated too far. Thus, in contrast with, for example, Newell and Simon (1972), Schank and Abelson did not focus on syntactic or formal aspects of information processing As one of the most important components of memory they hypothesized a procedure for recognizing repeated or similar event sequences. As a result of the operation of such a procedure, scripts, or generalized event sequences, are formed. Thus, a script is a predetermined, stereotyped sequence of actions that defines a well-known situation. Scripts allow for new references to objects within them just as if these objects had been previously mentioned. A script is made up of slots and requirements about what can fill those slots. Scripts can be activated (e.g., by mentioning or reading the script header) and instantiated (i.e., "... a copy of some of its details is made, with slots filled in by the known properties of the story at hand," Schank \& Abelson, 1977, p. 47). Human understanding is heavily script-based; every human is equipped with thousands of scripts. Scripts provide connectivity; specific individual events can almost effortlessly be related by applying scripted knowledge.

In the field of artificial intelligence, researchers trying to implement complex, real-life knowledge and everyday reasoning into computer systems see themselves faced with the "commonsense knowledge problem" (Dreyfus \& Dreyfus, 1986b). How should complex social knowledge be programmed into a computer in order to enable it to understand simple stories, like why the assertion "John loves Mary" could naturally result in a marriage proposal from the part of John (Rose, 1984)? Systems like PAMELA and PANDORA had a hard time in learning to explain and predict these types of events, even if supplied with large amounts of knowledge. In the years following its publication, the work of Schank and Abelson (1977) was heavily exploited by workers in the field of artificial intelligence trying to build systems that were able to understand everyday social situations (cf. Rose, 1984).

Nevertheless, the impact of the script approach has been larger for psychology than for artificial intelligence. A number of important studies and theoretical elaborations have been presented by, among others, Abbott, Black, and Smith (1985), Abelson (1981), Brewer (1986, 1987), Maki (1990), Mannes and Doane (1991), Nakamura, Graesser, Zimmerman, and Riha (1985), and Reiser, Black, and Abelson (1985). To a certain extent, this popularity of the script idea can be attributed to the fact that scripts are relatively high-level structures: the script 
concept is compatible with a number of underlying representational mechanisms, like production systems (Klahr, 1984) and connectionist networks (Feldman, 1989; Mannes \& Doane, 1991).

Generally, schemas and scripts are highly similar in nature; in fact, scripts are a specific kind of schemas, in that they (1) have a goal structure, (2) have an actor whom enacts the script, (3) consist of scenes sequenced in time. Most scriptrelated research has been performed in the domain of human text processing. From the early 1970s onwards, a number of researchers became interested in the way people understand complex verbal materials (e.g., Bransford \& Franks, 1972; Bransford \& Johnson, 1972; Garrod \& Sanford, 1983). Bransford and his colleagues (Bransford \& Franks, 1972; Bransford \& Johnson, 1972) found that subjects were often completely unable to comprehend ambiguously phrased stories about everyday activities, until the title (e.g." "washing clothes") was presented: From that moment onwards, problems were over. Furthermore, making inferences about subsequent or previous events in stories based on familiar situation-action routines did not require effortful elaboration at all, even if parts of the information were omitted (Bower, Black, \& Turner, 1979; Smith \& Graesser, 1981). Therefore, schemas and scripts were advanced as knowledge structures enabling these kinds of automatic expectations and inferences, which are computationally difficult to model, but generally very easy for human subjects. For example, Garrod and Sanford (1983) found that sentences that fit within a global topic - a notion closely resembling a script - are always processed quickly, whereas sentences that do not fit into such a topic are only processed quickly if the antecedent has already been mentioned in the previous sentence.

The script theory can easily explain why, with increasing time lapses, subjects tend to forget specific details of a particular scripted event, while other aspects are retained (Graesser, Woll, Kowalski, \& Smith, 1980), and why even some additional, unstated information might seep into the memory; this latter aspect was already reported by Bartlett (1932/1954), and subsequently by many other authors. As the script theory also makes a number of more detailed predictions concerning memory variables, a separate chapter (Chapter 5) will be dedicated to script based memory phenomena.

The actual existence of scripts is difficult either to prove or to falsify; howm ever, it is probably of significance to contrast seript theory with some other mechanisms for representing knowledge and understanding real world events, particularly approaches based on inductive reasoning (Holland, Holyoak, Nisbett, \& Thagard, 1986), mental models (Gentner \& Stevens, 1983; Johnson-Laird, 1983), situation models (van Dijk \& Kintsch, 1983), explanation based reasoning (Chi, Bassok, Lewis, Reimann \& Glaser, 1989; Mitchell, Keller, \& Kedar-Kabelli, 1986), case-based reasoning (Lancaster \& Kolodner, 1988; Kolodner \& Riesbeck, 1986), and analogy (Gick \& Holyoak, 1983; Spiro, Feltovich, Coulson, and Anderson, 1989; Vosniadou \& Brewer, 1987). The most important feature that distinguishes scripts from these other approaches is the absence of actual reasoning in script application. Activation and instantiation of an appropriate script is sufficient to understand complex real world events "immediately." Conversely, the other approaches precisely tend to stress the importance of reasoning. Therefore, scripts are not so much the theoretical opponents of mental models and casebased reasoning, as mainly applicable in other situations. Behavior in routine situations is often script-based, while behavior in non-routine situations often requires induction, case-based reasoning, or mental models. Consequently, scripts can be learned and applied without knowledge of the underlying mechanism, or even in the absence of any underlying mechanism. For example, con- 
cerning the restaurant script, children soon learn that "paying" is an "important scene, even if they do not know why, i.e., have no knowledge of the underlying economic mechanism. Similarly, people can learn ceremonies and rituals for which no underlying explanatory mechanism is available (cf. Ahn, Brewer, \& Mooney, 1992), and hence, reasoning is to no avail. Finally, scripts are highly domain specific structures, while approaches based on reasoning often focus on between-domain similarities to explain why something is happening. In fact, Newell and Simon (1972, p. 72) define problem as: "(a situation in which) someone wants something and does not know immediately what series of actions he can perform to get it." Effortless script application, in this view, can not even be considered a form of problem solving. However, programmers in the late $1970 \mathrm{~s}$ who were trying to program computers to solve simple everyday problems, like avoiding to get wet when it rains - i.e., situations in which humans probably would apply scripts - soon had to acknowledge that to solwe such deceptively simple problems, in fact enormous amounts of knowledge were necessary (cf. Rose, 1984). Indeed, the very idea of scripts was coined to explain why subjects were able to solve such problems effortlessly. As a consequence, the scientific notion of what constitutes a problem is currently broader than some 20 years ago: Nowadays, any non-trivial task, irrespective of the question whether the solution is immediately available to the subject or effortful problem solwing is required, may be considered a problem (cf. Boshuizen, 1989).

Thus, if scripts are domain-specific structures, that can be applied effortlessly in routine situations, and do not require extensive knowledge of the underlying explanatory mechanism, why not assume that in medical diagnosis a similar type of knowledge structure is employed? It seems not too far-fetched to assume that a physician who sees a patient with certain characteristics, symptoms, and complaints, and who knows immediately (or, at least, has a good hunch) what kind of disease this patient has, is applying a script. Therefore, we think, purely on theoretical grounds, that the notion of an illness script has. already a certain plausibility: the physician in question is evidently applying domain specific knowledge, this application is relatively effortless, and no reasoning is required (Schmidt \& Boshuizen, 1993). We will further elaborate on the issue of the illness script in a later section; at this point, an important difference between the actual function of a script in the sense of Schank and Abolson (1977; for reasons of convenience, we will call this a "classical" script), and the presumed illness scripts, should be discussed.

The question concerns the activation of scripts. In most script-based studies, this activation is taken for granted, or at least not considered a major problem. In real-life, people usually know what kind of activities they are engaged in, and what purposes these activities serve. Similarly, in most studies, script activation. generally is accomplished by mentioning the script title, e.g." "going to a restaurant," or "visiting the dentist", thus, the activation of an inappropriate script is almost inconceivable. Indeed, it is difficult to write a story that can be interpreted according to two different scripts. Anderson, Reynolds, Schallert, and Goetz (1977) constructed two such stories, one that could be interpreted as either a prisoner in his cell planning to escape, or a wrestler trying to break the hold of his opponent, and another one that could be interpreted as either a social evening of cards, or a woodwind ensemble's rehearsal. However, both stories have a rather artificial flavor. In a medical diagnostic situation, on the other hand, activating the appropriate illness script (i.e., finding the correct diagnosis) is usually the core of the problem. Thus, though classical scripts and illness scripts may be represented in a similar way, i.e., are structurally alike, issues of access 
and retrieval are extremely important for illness scripts, while they are almost trivial for classical scripts. As such, research on analogical problem solving (e.g., Gick \& Holyoak, 1983; Holyoak \& Thagard, 1989; Spiro, Feltovich, Coulson, \& Anderson, 1989), in which also finding a proper analog is an important topic, may be relevant to illness scripts. "The same applies to studies on the role of situational and contextual factors in knowledge activation, such as the study of Godden and Baddeley (1975) on learning and recall of lists of paired associates in two environments: on land and under water. Reinstating the usual context in which a particular script is activated may facilitate current script activation, just like paired associates learned under water may be recalled better under water. In fact, this usual context may even become part of the script knowledge; in illness scripts, this means that situational aspects and patient characteristics gradually become part of the illness script (cf. Hobus, Boshuizen, \& Schmidt, 1989; Hobus, Schmidt, Boshuizen, \& Patel, 1987) and may eventually play an important role in script activation. This contextualization of illness scripts may also be responsible for the case-specificity effects, i.e., the low correllations between diagnostic performances on two different cases of the same disease, that are often reported in medical expertise research (e.g., Elstein, Shulman, \& Sprafka, 1990).

Apart from the importance of factors that contribute to script activation, another issue that remains unaddressed by the classical approach of scripts is even more relevant to illness scripts: their development. Classical scripts are concerned mainly with well-learned, reall-life activities, of which even young children may be highly knowledgeable: for example, many a parent will admit that children may possess a highly adequate script for visiting a dentist. However, adequate disease knowledge, whether or not it is represented as illness scripts, requires many years of formal training and practice to develop: Only experts possess full-fledged medical diagnostic knowledge. To trace the development of this knowledge - and, of course, the nature and development of expert knowledge in general - a large number of studies have been performed, in a number of domains, though with an emphasis on medicine. Thus, before discussing in more detail the structure and function of illness scripts, two sections will be dedicated to expertise research, one to expertise in general, and one to medical expertise. This expertise research has contributed substantially to our current conceptualization of the illness script.

\section{EXPERTISE RESEARCH: GENERAL OVERVIEW}

If a price were to be awarded for offering the most concise and adequate definition of what an expert is, Norman (1988) would be a prominent candidate, as he states, (wording a little bit adapted): "An expert is someone who has seen it all before." This definition invokes the image of an expert as not only someone who has a lot of experience in a particular field, but also someone whom can be trusted, who won't easily be disturbed or surprised, and who has a solution or answer available to any conceivable problem in the domain before lesser gods even have begun to understand it. In economic terms, expertise is a valuable and rare good, as manifested by experts receiving high wages. For some decades now, psychologists are trying to analyze experts" behavior and knowledge, sometimes motivated by a desire to study the basic properties of human information processing and problem solving, sometimes motivated by the hope to find means to accelerate expertise development. Presently, the results might be summarized 
by saying that experts have much experience and show superior performances in their domain, but do not truly differ from "ordinary" people as far as basic abilithes or capacities outside their domain are concerned. In addition, there is little evidence that the development of expertise can really be speeded up, at least not for subjects who are already engaged several hours a day in activities aimed at expertise acquisition, as part of an educational program. In the present section, we will try to give a cognitive-psychological account of expertise in general, with an emphasis on the features that experts in different domains have in common, rather than on inter-domain difierences.

As far as content is concerned, expertise studies are, generally, highly similar to related work on knowledge representation and problem solving: A salient feature of expertise research is, however, that subjects at two or more levels of experience are compared while performing a particular task. In its simplest form, the performance of a group of experts in a particular domain is compared to that of a group of novices. In more elaborate designs, laypeople, intermediates, and outstanding specialists may also be included, and the nature of the study may be (partly) longitudinal, rather than cross-sectional (cf. Wohlwill, 1973).

Several domains have been subjected to expertise research: chess (Chase \& Simon, 1973; Ericsson \& Smith, 1991; de Groot; 1946/1978), algebra (Novick, 1988), geometry (Koedinger \& Anderson, 1990), elementary physics (Chi, Feltovich, \& Glaser, 1981; Clement, 1991; Elio \& Scharf, 1990), bridge (Charness, 1979, 1989; Engle \& Buckstel, 1978), computer programming (Adelson, 1981; Ehrlich \& Soloway, 1984), industrial troubleshooting (Schaafstal, 1991), military strategic thinking (Forsythe \& Barber, 1992), psychiatry (Cantor, Smith, French, \& Mezzich, 1980; Nurcombe \& Fitzhenry-Coor, 1982), cardiology (Feltovich, Johnson, Moller, \& Swanson, 1984; Johnson, Durán, Hassebrock, Moller, Prietula, Feltovich, \& Swanson, 1981), neurology (Gale \& Marsden, 1983; Snoek, 1989; Wortman, 1966), radiology (Carmody, Kundel, \& Toto, 1984; Garland, 1959; Lesgold, 1984), writing (Scardamalia \& Bereiter, 1991), auditing (Tubbs, 1992; Vaatstra, Boshuizen, \& Schmidt, 1993), music (Ericsson, Krampe, \& Tesch-Römer, 1993; Sloboda, 1991), and history (Wineburg, 1991), and this list is by no means exhaustive. Especially medical expertise in has been an extremely productive field of study. The next section will be completely dedicated to medical expertise; in the current section, relevant research from the other domains will be reviewed.

Since the pioneering work of de Groot (1946/1978) on expertise in chess, this game has gained large popularity among researchers, almost to the point of becoming a "Drosophila for cognitive psychology" (Charness, 1989). It was the research into chess that first pointed towards the importance of memory, rather than search, for expert performance (Chase \& Simon, 1973; De Groot, 1946/1978). Human capabilities to perform the in-depth search required for chess are very limited, and therefore can not be the source of human expertise in chess. Master chess players have a tremendous capacity for recognizing relevant chess patterns, and it is this capacity that is responsible for their achievements. Of course, almost always some amount of search is involved, but the moves to which this search process is applied are selected by a recognition process. In contrast to present expertise research, which is often performed in an educational context,

4 Actually, the French psychologist. Alfred Binet was the first to conduct a survey into (especially blindfold) chess expertise, within the framework of wisual memory. However, being only superficially in touch with the game, and with a psychological theory to incorporate chess playing behavior still completely lacking. Binet either missed relevant obserwations or was unable to interpret them (cf, de Groot, 1978). 
de Groot (1946/1978) was more interested in superior chess thinking and playing than in the actual development of chess expertise. Hence, he did not include novices or even "true" intermediates in his studies (except for the recall studies), but compared grandmasters (including the 1935 World Champion Dr. Max Euwe) with, at lowest level of comparison, skilled club players (including De Groot hirnself). At the time de Groot performed his research, ELO-ratings were not yet invented; therefore, quantitative data about the chess strength of de Groot's subjects are not available. However, without doubt these chess players were by any standards at least highly skilled; consequently, the de Groot study provides no information about the initial stages af chess expertise development.

Though expertise research is basically developmental research, and thus longitudinal study designs would be most appropriate (cf. Wohlwill, 1973), actually almost exclusively cross-sectional designs are used (for a notable, albeit $\mathrm{N}=1$ exception, see Charness (1989)). Obviously , the underlying reason is not difficult to guess: acquiring expertise is an extremely long-winded affair. For example, Boshuizen (1989) estimates that a total of 21,600 hours are spent on study and clinical practice before one may call oneself an experienced family physician. Charness (1989), who reports having tested a particular Canadian chess player twice, in 1978 and 1987 (the above-mentioned longitudinal $\mathrm{N}=1$ study), calculated that this subject had in the meantime invested about 4,500 hours in playing chess. In those nine years, he advanced from a moderately skilled chess player to the seventh strongest player in Canada: His ELO-rating increased from 1,570 to 2,423 , being a shift of four standard deviations. Furthermore, Ericsson, Krampe, and Tesch-Römer (1993) estimated total hours of practice in playing the violin of future top-level violinists, good violinists, and music teachers: At age 18, these values amounted to 7,410 hours, 5,301 hours, and 3,420 hours, respectively. It is probably redundant to remark that most research grants cover only a tiny fraction of the time scale involved in becoming an expert in most domains: As a practical rule of thumb, it is sometimes stated that one is an expert after ten years of intense practice in a field (cf. Simon \& Chase, 1973).

Generally, it is not sufficient to compare experts and novices only on task outcomes. That experts perform better than novices is not a telling conclusion: Usually, a more fine-grained level of analysis is required to reveal the underlying differences that can account for expert-novice differences in task outcomes. Three types of experimental paradigms are employed in expertise research (cf. Ericsson \& Smith, 1991; Olson \& Biolsi, 1991; Gilhooly, 1990); (a) verbal protocols of thinking aloud during problem solving, probing of the problem representation, retrospective reports, or explanations of certain problem aspects; (b) knowledge structure probing tasks, like concept sorting, similarity rating, intermittent probing of the problem representation, free associations, and interview studies; (c) recall or recognition studies, with the recall or recognition task either being announced before the study task is administered (intentional memory) on not (incidental memory). Within each of these paradigms, a large number of studies has been performed. Though the results of these studies by no means unequivocally point into the same direction, a number of general conclusions can presently be drawn, particularly if an effort is made to overcome differences in the terminology employed by different authors to phrase conclusions with largely the same flavor. Briefly, expertise research results can be summarized as follows:

1) Experts' knowledge is better structured and organized than non-experts' knowledge. This superior structuring and organization of experts' knowledge is conceptualized as and accounted for by a large repertory of schemas (Gil- 
hooly, 1990; Glaser, 1986; Rumelhart \& Norman, 1978; VanLehn, 1989), more efficient chunks (Engle \& Buckstel, 1978), or by compilation of production rules in a production system (Anderson, 1983, 1987). As a consequence, experts are able to show pattern recognition behavior or "immediate comprehension" (Ericsson \& Smith, 1991 ), to remember new information in their domain better (Chase \& Ericsson, 1981), to display inference patterns that can be characterized as forward reasoning (Patel, Evans, \& Groen, 1989; Patel \& Groen, 1986a), to have solution procedures available as one functional unit, to have better problem representations (Chi, Feltovich, \& Glaser, 1981; Novick, 1988), to make more inferences from the data (Patel \& Coughlin, 1985; Patel \& Frederiksen, 1984), to remember more critical information (Coughlin \& Patel, 1987; Norman, Brooks, \& Allen, 1989), or to substitute knowledge for reasoning (Waldrop, 1984). The availability of well-tuned, flexible schemas makes it possible to reserve working-memory capacity for additional processing; as a consequence, experts can handle noisy data (Glaser, 1986). Experts performance breaks down, however, if meaningless patterns or random data are presented (Engle \& Buckstel, 1978; de Groot, 1946/ 1978; Norman, Brooks, \& Allen, 1989). Finally, experts are reported to have superior incidental, but not intentional, memory for information from their domain (Norman, Brooks, \& Allen, 1989).

2) Apart from being better organized and structured, experts' knowledge is also conditionalized (Bransford et al, 1989; Boshuizen, 1989; Turner, 1988), i.e., tuned to the situations in which it is of use (Rumelhart \& Norman, 1978).

3) Though experts also have quantitatively more knowledge than novices, there is no evidence that there are differences in basic capacities between subjects at different levels of experience (cf. Ericsson \& Smith, 1991). This led Salthouse (1991) to conjecture that expertise is the process or processes involved in circumventing the common constraints of human information processing. Indeed, experts are, at least on routine tasks, but probably on all tasks, faster and more accurate than novices (Charness, 1979; Norman et al., 1989), and less susceptible to time constraints on processing (Schmidt \& Boshuizen, 1993). One study (Norman, 1992) even reports a gradual decline in the gize of physicians' knowledge base after graduation; though this may be an artefact of a cross-sectional research design, it supports the supposition that expertise can not be solely explained by incremental storage of knowledge ellements in an ever-growing knowledge base.

4) In many cases, expert knowledge is at least partially of a procedural, implicit, or tacit nature (Ehrlich \& Soloway, 1984; Gick \& Holyoak, 1983; Sternberg \& Caruso, 1985). Though Elstein et al. (1978) report that physicians do not use patient cues that are difficult to verbalize, this is by definition an issue that can not be settled by studies using subjects' verbal reports as data. The possible role of tacit knowledge, initially broached by Polanyi (1958), is currently attracting increased interest (e.g., Boshuizen and Schmidt, 1991; Reber, 1989; Schmidt \& Boshuizen, 1993b; Sternberg, 1994; VanLehn, 1991). The finding of Hobus, Hofstra, Boshuizen, \& Schmidt (1988) that expert physicians use contextual factors in activating diagnostic hypotheses could also be interpreted as an indication that they are using tacit or implicit knowledge. 
5) Presently, evidence is accumulating that rule-based problem solving is a feature of novice, rather than expert, behavior (e.g., Allen \& Brooks, 1991; Dreyfus \& Dreyfus, 1986b). This is at variance with practically all attempts to implement experts" knowledge in expert systems, and it is also implicitly or explicitly inconsistent with many psychological approaches to expertise (e.g., Holyoak \& Thagard, 1989). However, encouraging or inducing novices to learn and use expert rules, an approach that is advocated by so-called overlay models, and that seems also to be a logical consequence of the Newell and Simon (1972) view on problem solving, does not lead to increased performance (cf. Holyoak, 1991). Similarly, Elshout (1983) expresses caution against students showing pseudo-expert behavior, i.e., mimicking expert behavior without disposing of sufficient knowledge, while D. Evans (1989) also seems to be aware of this problem when he addresses the issue of "form learned before content." Learning large numbers of rules does not make one an expert. The notion that expertise is principally not rule-based has stimulated the development of hybrid models and expert systems based on connectionist networks (e.g., Holyoak, 1991; Schyns, 1991).

6) Especially in the medical domain, but also in other fields, an intermediate effect is often reported: an inverted- $U$ relationship between expertise level and a number of dependent measures, including recall verbosity (Boshuizen, 1989), size of the problem representation (Schmidt, Boshuizen, \& Hobus, 1988), amount of reasoning during problem solving (Kaufman \& Patel, 1988), extensiveness of search (Saariluoma, in: Charness, 1991), number of categories employed in a categorization task (Murphy \& Wright, 1984; Norman, Rosenthal, Brooks, and Muzzin, 1989), and even performance (in radiology, of. Lesgold, 1984). Intermediates seem to be especially susceptible to constraints on information processing time, while neither novices' nor experts' performances are affected by it (Schmidt \& Boshuizen, 1993); if no time constraints are imposed, intermediates tend to be slower than both experts and novices (Norman, Brooks, \& Allen, 1989).

Some caveats have to be made, however. For example, that experts display forward reasoning has not always been confirmed (Patel \& Groen, 1986a): If information becomes only sequentially available, like at interactive tasks, working forward may be impeded. Moreover, in some studies, forward reasoning is explicitly associated with novice, rather than expert, behavior (cf. Carlson, Khoo, Yaure, \& Schneider, 1990; Lancaster \& Kolodner, 1988). According to Koedinger and Anderson (1990), experts show forward behavior only on relatively simple tasks. It seems safe to conclude that working forward is a consequence of the development of expertise, and as such is displayed by experienced subjects on particular tasks (cf. Gilhooly \& Green, 1988). Furthermore, in some domains, like historical problem solving, schemas may play a less important role (cf. Wineburg, 1991), and may expert behaviour indeed be characterized by deeper, more thorough, search. The point is that expertise researchers have generally used problems that are difficult for novices, but routine for experts (e.g., Anzai, 1991; Chi, Feltovich, \& Glaser, 1981), while few studies have investigated the behavior of experts on - even for them - difficult problems. Thus, as Wineburg (1991) argues, "expertise may not only consist of what experts know, but also of what they are able to do when they do not know." Problems may be difficult because they exceed available computational resources; by using a sophisticated strategy of incremental planning, experts may be able to overcome these limita- 
tions (Kuipers, Moskowitz, \& Kassirer, 1988). This also raises the issue whether adaptive expertise, or domain-free facets of expertise, exist, i.e., whether the capacity to transform non-routine, ill-structured problems into familiar ones, constitutes a separate ability (Glaser, 1990; Schraagen, 1993).

An assumption implicit in most expertise research is that expert behavior is the norm. Little attention has been paid to the origin of experts' errors, or to inter-expert differences. This point is nicely illustrated by Johnson's (1988) recommendation "... to hire the cheapest, not the best expert you can find": Apparently, experts are all alike. Unlike most expertise studies in cognitive psychology, behavioral decision theory tends to focus on experts' failures, particularly if expert performances are inferior to those of statistical models based on these experts" own behavior (Camerer \& Johnson, 1991; Elstein et al., 1990). However, the behavioral decision theory provides a normative model for making decisions in difficult diagnostic or treatment situations; it does not tell anything about the actual process of diagnostic hypothesis generation. Thus, though the behavioral decision theory may be able to distinguish between good and poor expert decisions and solutions, it can not explain why an expert - or, for that matter, a novice succeeds or fails in activating the correct diagnostic hypothesis for a particular case. As we are presently mainly interested in the development of expertise and expert knowledge structures, we will not elaborate on inter-expert differences, but concentrate on expert-movice differences.

\section{STUDIES IN MEDICAL EXPERTISE}

For a number of reasons, expertise research has to a large extent been dominated by studies in the medical profession. One of these reasons may be the important role of formal education and training in this domain. Though it is probably possible to be a chess expert at age four, a violinist expert at age eight, a mathematics expert at age 12 , and a soccer expert at age 16 , it is hardly conceivable that one could be a medical expert at any of these ages, or even at the age of 20 or 24. In addition, unlike chess, music, or mathematics, it is not possible to become an expert physician merely by self-study and home practice. Furthermore, as becoming a medical expert is an extremely costly and long-winded affair, it is obvious that successful attempts to accelerate or enhance the development of this expertise in students, might easily lead to a quick recovery of the invested costs. Thus, expertise research in medicine often serves a clear educational purpose. Besides, since life and death are often involved, medical expertise has a luigh societal impact: The consequences of a physician making a mistake may be much more serious than, for example, a chess player, mathematician, or sociologist, making a mistake - at least as far as the immediate consequences are concerned. For these reasons, medical expertise has received more attention than expertise in other domains.

Furthermore, expertise in the medical domain has also been an attractive subject for cognitive psychologists (D. Evans, 1989). First, medicine is characterized by a relatively well developed language for concepts, even for mainly perceptual concepts, like those employed in dermatology or otolaringology. This is in contrast with domains, such as music or most physical sports, in which knowledge implicit in motor schemas plays a more important role. Second, unlike in mathematics or logic, there is no formal problem solving theory in the medical domain, and certainly not in clinical medicine. Thus, the content and the process of problem solving in medicine can not be separated: For example, if an incorrect 
diagnosis is proposed as the solution of a diagnostic problem, it will often be impossible to analyze why the correct solution was missed. In this respect, medicine is described as similar to both chess (Anzai, 1991) and bridge (Charness, 1989), in which it is also difficult to tell why a superior move or play was not found. Third, the medical domain is sufficiently well defined and constrained to enable, in most cases, objective judgment of the quality of the outcome of the processes. In this respect, medicine differs from broader fields like economics, history, or sociology, in which problem solving aften requires application of a considerable amount of real-world, general knowledge, and solutions are difficult to compare on a single poor-good dimension.

Though expertise research in medicine has a history of only a few decades, ever since the beginning of modern medicine, physicians have formulated notions about how to arrive at sound diagnoses. Da Costa (1864, in: Schwartz \& Griffin, 1986), for example, already stated that: "... the detection of disease is the product of the close observation of symptoms, and of correct deduction from these symptoms... When ... the symptoms of the malady have been discovered, the next step toward a diagnosis is a proper appreciation of their significance and of their relation toward each other. Knowledge, and abowe all, the exercise of the reasoning faculties are now indispensable..." This quotation is probably one of the first efforts to formulate a theory of medical diagnostic expertise. In fact, the concept of "medical diagnosis" in its present sense, i.e., as an explanation of a pattern of symptoms on basis of an underlying biomedical model, has a relatively short history; up until the end of the eighteenth century, physicians were essentially ignorant of the true causes of most diseases. Disease theories and disease therapies often outrivaled each other in absurdity (cf. Schwartz \& Griffin, 1986). However, by the end of the nineteenth century, accurate diagnosis was already the most important goal of medicine. Medical diagnosis was by then seen as very similar to scientific classification, especially to that in botany and biology. And, as Da Costa's citation attests, even primitive theories about psychological processes behind medical diagnosis began to emerge.

However, a century was to pass before problem solving in medicine became a subject of scientific study. Given the success of the Newell, Shaw, and Simon (1958) and Newell and Simon (1972) endeavours, it could be expected that it would not take long before the findings of these investigators would be applied to problems in knowledge-rich domains, such as medicine. Hence, psychologists as well as physicians, have been engaged in investigating the role of general problem solving methods in physicians' professional behavior, and in disentangling the possible relationships between expertise level and formal aspects of a consultation. This approach is referred to by Elstein et al. (1978) as the "primacy of process over content" view. In line with the spirit of the times (e.g., Miller et al., 1960), it was emphasized that physicians were primarily expected to be good problem solvers. The perception that medical science was developing at a vertiginous speed, which would render much of the current knowledge obsolete within five or ten years, contributed to this conviction. Though the impact of this view has considerably declined since the 1970 s, even in relatively recent work the distinction between thinking or problem solving processes on the one hand and medical content knowledge on the other hand is maintained (e.g., Cutler, 1979; Gale \& Marsden, 1983; Snoek, 1989). For example, Gale and Marsden (1983) distinguish between cognitive processes and skill profiles; in addition, they identify and define 14 separate "thinking processes," though they fail to discriminate between medical students and physicians on basis of these processes. Even more recently, 
Snoek (1989) assigns a major role to better problem solving, i.e., a repertory of valid heuristics and thinking methods, in neurological expertise.

Cutler's (1979) work is particularly illustrative in this respect. He presents a rather curious mix of general problem solving methods and specific knowledge in clinical problem solving. "All the physician did was take a history, do a physical examination, and order a few basic tests. During this fluid data-gathering process, he kept filtering clues through his information base. He formed, then rejected or accepted hypotheses based mainly on his knowledge, logic, judgment and experience" (p. 3). A bit further, when explaining why experienced physicians take less time than inexperienced physicians: "... rather, he has been mas" tering the skill of problem solving by quickly grouping clues, intelligently selecting key questions, examining where indicated, going off on appropriate tangents, and taking the correct turms at branching points. Thus, he reaches conclusions more rapidly. He may begin this process on meeting the patient and shaking his hand, even before starting the history, and be well along toward solving the problem on first hearing the chief complaint." Cutler presents 19 what he calls "problem solving methods"; actually, however, these constitute a remarkable mixture of tools and aids (e.g., problem lists, key clues), general strategies (e.g., early hypothesis, branching technique, suppression of information, anatomical approach), data summarization methods (e.g., clusters, syndromes, pattern building) and representational formats (e.g., dendrograms, Venn diagrams). In many cases, these problem solving methods are in fact descriptions of physicians" behavior (e.g., data gathering, pattern recognition).

At the time Newell and Simon (1972) published their influential work on human problem solving, Arthur Elstein and his colleagues (Elstein et al, 1978) had already started an extensive study that aimed at disclosing the relevant charac teristics of outstanding physicians' medical problem solving. Their key-hypothesis was that excellent physicians could be distinguished from their less outstanding colleagues by their superior problem solving qualities, i.e., that formal process variables play a critical role in medical expertise. However, in several experiments, Elstein et al. (1978) failed to find evidence for this hypothesis. Succinctly, their conclusion boils down to: "... results were nonsignificant - sometimes dramatically so" (p. 160). However, nonsignificant does not necessarily mean insignificant, and Elstein et al. were able to report a number of interesting results which, if they sound common now, may very well owe their current familiarity to Elstein et al.'s work. For example, clinical competence turned out to be highly case-dependent; the quality of a particular physician's problem solutions shows no consistent trend over different problems. Apparently, medical expertise is not a one-dimensional concept, but should always be assessed in relation to a particular, relatively confined domain of problems. Maybe the actual situation is even worse: When asked to judge biopt-slides of patients suffering from Hodgkin's disease, Einhorn (1974) reports an inter-judge reliability of 27 , and an intra-judge reliability of .63. These values may be considered discouragingly low.

Other remarkable findings of Elstein et al. (1978) were that, in contrast to medical teachers' general recommendations (e.g., Wilkins, 1970), expert physicians tend to generate diagnostic hypotheses in a very early stage of the clinical encounter, when only a limited amount of information is yet available; and that the total number of these early hypotheses generally ranges from 5 to 7 and remains constant throughout the remainder of the clinical encounter. That early hypothesis generation is an important feature of expert behavior was confirmed by Neufeld, Norman, Feightner, and Barrows (1981): If experts take into consideration the correct diagnosis during the first 5 minutes of a consultation, this hy- 
pothesis becomes definite in $95 \%$ of the cases; however, has the proper diagnosis not yet crossed the physician's mind at that point, there is a $95 \%$ probability that it will be missed. The finding of a quantitatively constant hypothesis set, independent of level of expertise, has been corroborated several times (Johnson et al., 1981; Neufeld et al., 1981, Weber et al., 1993). Weber et al. (1993) also found consistent interindividual variation in the size of this initial set. The role of this small set of hypotheses is to provide a functional problem space in which subsequent problem solving can take place, i.e., to reduce the original virtually unlimited problem space to a manageable size. However, the quality of the generated hypotheses, or, for that matter, of this reduced problem space, is higher for experts than for novices (Elstein et al., 1978; Johnson et al., 1981; Neufeld et al., 1981). For novices, it is not uncommon to exhibit extremely data-driven diagnostic behavior, which may result in "hypothesis hopping": with each additional finding, a new diagnostic hypothesis is generated (Johnson et al., 1981). A characteristic feature sometimes reported of experts' hypothesis generation is a gradient from general to specific in the sequence of proposed hypotheses (Weber et al., 1993), while other researchers were unable to discover this tendency (Barrows, Norman, Neufeld, \& Feightner, 1982; Elstein et al., 1978). Generally, the findings of Elstein et al. (1978) were also confirmed in the domain of nursing (Tanner, Padrick, Westfall, \& Putzier, 1987).

According to Elstein et al. (1978), a psychological model of the diagnostic process can be built around four important activities: cue acquisition, hypothesis generation, cue interpretation, and hypothesis evaluation. Of these, hypothesis generation is the critical factor. If the accurate diagnosis is taken into consideration, it is seldom rejected (cf. Norman, 1988); diagnostic errors are strongly associated to absence of the correct hypothesis. Though reasoning errors like confirmation bias (Boshuizen \& Schmidt, 1990; Gerritsma \& Smal, 1982; Norman, 1988) and premature closure (Patel, Evans, \& Kaufman, 1989; Wilkins, 1970) are reported for physicians, it seems unlikely that they constitute an important source of diagnostic errors or less than optimum solutions (Elstein et al., 1978). These apparent reasoning errors should be viewed as a consequence of the failure of subjects to introduce new hypotheses in later stages of the diagnostic process and their reluctance to reject unlikely but cherished hypotheses (Wagenaar, 1987; Weber et al., 1993), even up to the point of continuing an evidently incorrect treatment (Eistein et al., 1978).

The generally rather disappointing results of Elstein et al. (1978) painstakingly revealed how much medical problem solving, particularly clinical problem solving, differs from solving relatively simple logical puzzles or arithmetic problems. The view that knowledge and awareness of the applicability of that knowledge are the key ingredients for medical competence pervades the work of almost all authors in the field. Content-independent aspects of problem solving in medicine probably do not constitute an independent research topic at all, but should be considered an epiphenomenon of applying medical knowledge. For example, Elstein et al.'s (1978) finding that overinterpretation (i.c., the inclination to interpret new information as supportive of a current diagnostic hypothesis, and the reluctance to reject such an hypothesis in light of disconfirmative information) is the most often occurring mistake, should be viewed as a cognitive, knowledge-dependent error, and not as a consequence of a wrong motivation or a poor reasoning style: Subjects simply fail to think of a better alternative hypothesis (cf. J. St. B. Evans, 1989). Medical expertise research should be directed at disclosing the structure and application of that knowledge, and not at the problem solving process pure. 
That medical knowledge and its application play a key role in medical expertise does not imply that search for data, interview techniques, physical examination skills and capacity for communicative support are irrelevant tools in a physician's repertory; rather, on basis of these aspects, it is difficult or impossible to discriminate between experts and non-experts (Neufeld et al., 1981). Other general characteristics that do discriminate between medical experts and nonexperts can be interpreted as a consequence of differences in knowledge structures between experts and novices. For example, the ability of medical experts to use forward reasoning (Patel \& Groen, 1986a) and to apply strong problem solving methods (Groen \& Patel, 1985) points towards the use of qualitatively different knowledge structures.

The idea that knowledge, rather than problem solving methods, lies at the roots of medical expertise, raises questions about the nature of this knowledge. Medical knowledge is not a unitary construct; often, a distinction is made is between biomedical knowledge and clinical knowledge. Biomedical, or medical basic knowledge concerns mainly the anatomy and physiology of the luuman body, including its possible malfunctions. Thus, sciences like biology, biochemistry, chemistry, physiology, pathophysiology, histology, and epidemiology are all involved. Clinical knowledge is defined as knowledge of the attributes of ill people (Boshuizen \& Schmidt, 1990). Some authors argue that the most important - if not the only - component in medical diagnostic expertise is the correct application of biomedical knowledge (e.g., Feltovich \& Barrows, 1984; Feltovich, Coulson, Spiro, \& Dawson-Saunders, 1992; Hassebrock \& Prietula, 1992; Kuipers \& Kassirer, 1984; Swanson. Feltovich, \& Johnson, 1977). This view is shared by researchers from the medical domain (e.g., Paycha, 1955). For a number of years, a discussion is going on about the role of this knowledge in clinical expertise, i.e., in diagnosing and treating actuall patients. There is considerable support for the hypothesis that this knowledge is in fact scarcely used by experienced physicians in practical clinical situations (Boshuizen \& Schmidt, 1992; Boshuizen, Schmidt, \& Coughlin, 1988; Clancey, 1983; Patel \& Groen, 1986a; Patel, Evans, \& Groen, 1989; Schmidt \& Boshuizen, 1990; Schmidt \& Boshuizen, 1993). "The finding of Norman, Rosenthal, Brooks, and Muzzin (1989) of a positive relationship between speed and accuracy of experts' diagnoses, appears also inconsistent with the view that expert diagnosis is based on elaborate biomedical reasoning. On the other hand, biomedical knowledge may play a more prominent role in the interpretation of laboratory values (ci. Norman, Trot. \& Brooks, 1992). Elstein et al. (1978) reported that their subjects ere taking pathophysiological processes into consideration in about one out of three cases. According to Patel and Groen (1986b), biomedical knowledge and clinical knowledge are two worlds apart: biomedical knowledge is not used in generating causal explanations of a clinical case, while clinical medical education is not an effective means to increase "cognitive" knowledge (cf. Jones, Cason, \& Cason, 1986). Johnson et al. (1981) suggest that biomedical knowledge may play a more important role in medical fields in. which the major disease states can be described in terms of gross features of anatomy and physiology, like congenital heart diseases. Conversely, extensive biomedical knowledge may be of little help in diagnosing diseases for which only incomplete models of the underlying anatomical or pathophysiologicall processes exist; these diseases can only be diagnosed by clinical knowledge.

Findings that in many cases, biomedical knowledge is not actively applied by experienced physicians, do not imply that biomedical knowledge can be completely dispensed with in the process of acquiring medical expertise: clinical expertise might be a consequence of repeated application and subsequent compilation of 
biomedical knowledge (e.g., by a chunking or encapsulation process, cf. Schmidt and Boshuizen, 1993). An appealing consequence of this hypothesis is that it provides a nice opportunity to account for intermediate effects: During the earlier stages of expertise development, elaborate chains of reasoning are a necessary ingredient of medical problem solving, while in later stages, shortcuts and chunks are formed, and consequently, verbal protocols become less dense. However, actual evidence for the hypothesis that clinical expertise develops (exclusively) as a consequence of chunking or encapsulation of biomedical knowledge is still wanting, while some authors (e.g., Patel, Evans, \& Groen, 1989) seem to be convinced that it is not a fruitful approach. In the field of medicine itself, lore tends to assign a separate status to the "clinical look" (cf. Snoek, 1989) or the "bedside logic" (Cutler, 1979), as opposed to formal, biomedical reasoning and general problem solving methods. The problem with expressions like clinical look is that they do not clarify anything; furthermore, the existence of a clinical look or bedside logic does in itself not prove that they are not a consequence of compiled biomedical knowledge: it could be feasible that compiled biomedical knowledge manifests itself as the clinical look. However, as experienced physicians use elaborated clinical, rather than biomedical, knowledge to diagnose difficult, atypical cases (Boshuizen and Schmidt, 1990), the clinical look probably does not develop from biomedical knowledge, but from repeated application of clinical reasoning,

In summary, whether or not the origin of medical expertise can be found in application of medical basic knowledge, it seems highly unlikely that this knowledge plays a key role in diagnosis and treatment, the more so for cases the physician has experience with (i.e., "routine" cases). Physicians can diagnose and treat patients, even if a biomedical model of the underlying disease is completely or partly lacking. In addition, Spiro, et al. (1989) report that even expert physicians' biomedical models may sometimes include misconceptions, though this does not prevent them from making correct diagnoses. It is probably reasonable to assume (Feltovich, 1994, personal communication) that biomedical knowledge has a constraining function in medical diagnosis, providing an ultimate framework within which every explanation should be fitted.

An alternative approach that does not make assumptions about the use of biomedical knowledge is the view that clinical expertise, and especially diagnostic expertise, is essentially a form of categorization behavior. In this view, experts have more differentiated categories, or a larger pool of previous instances, against which a new case can be compared. Evidence in favor of a prototype representation of disease knowledge can be found in Bordage and Zacks (1984) en Genero and Cantor (1987). According to Bordage and Zacks (1984), physicians diagnose a case by performing a scan over all disease prototypes stored in their memory, with the objective of obtaining a fit. Genero and Cantor (1987) showed that exposing expert psychiatrists to a few category exemplars was sufficient to elicit a standard typicality effect in diagnostic judgments. Evidence that previous instances may be stored and retrieved was found by Elstein et al. (1978): The physicians in this study reported imagery of prior patients in $29 \%$ of cases. Explicit proponents of the view that diagnosis is instance based are Lee Brooks and his colleagues (Norman, Brooks, and Allen, 1989; Brooks, 1987; Norman, 1988). In this view, the use of previous instances is restricted to experts or experienced subjects (Dreyfus \& Dreyfus, 1986a, 1986b; Schmidt, Norman, \& Boshuizen, 1990); novices, on the other hand, do employ categorization rules. Norman, Brooks, and Allen (1989) found that the quality of current dermatological diagnoses can depend on the specific composition of a set of prior pictures of dermatological abnor- 
malities. The finding of Norman, Rosenthal, Brooks, and Muzzin (1989) that experts make proportionally about the same number of errors on typical cases, compared to atypical cases, than non-experts, is difficult to reconcile with an abstract prototype representation of diseases, but not with an instance-based view. A problem with many of these studies is that they originate from the field of dermatology, probably a medical subdomain that draws heavily on visual perception (e.g., Cutler (1979) refers to the situation in dermatology as: "What you diagnose is what you see"). Thus, it may be difficult to generalize these findings to medicine as a whole. There are also other considerations why pure similarity based learning, i.e., the use of instances without additional abstractional mechanisms or explanations, may not be an optimum strategy. For example, subjects are confronted with an induction problem: Which features of the presented cases are necessary constraints, which not (cf. Ahn et al., 1992). Retrieving previous cases from memory may be a function of the availability heuristics defined by Kahneman and Tversky (1974); these authors showed that the results of such operations are apt to be unreliable. There is also the associated risk of cluttering memory with irrelevant and possibly harmful information. Van Rossum, Bender, and Meinders (1991) and Van Rossum, Briêt, Bender and Meinders (1990) found that diagnoses of previous patients are often directly associated in memory to salient biographical features of these patients, like the fact that someone was employee on an offshore oil rig, regardless whether these features are related to the disease or not. If they are not, similarity based diagnosis of a subsequent case may not lead to optimum results. Though they did not address the issue in a diagnostic context, Hassebrock and Prietula (1990) also provided evidence that physicians often remember conspicuous, but irrelevant contextual details, while clinical findings are often either forgotten or reconstructed on basis of general memory for the disease in question. In summary, though physicians may use prior patients in the diagnostic process, it is likely that they do so only in a minority of cases, and, after being presented with two or more cases of the same disease, are highly inclined to form a general picture of a prototypical patient with that disease, with the non-communal features filtered out.

However, some researchers are of the opinion that categorization, whether on basis of prototypes or on basis of instances, should not receive too much em. phasis in medical diagnosis. For example, Feltovich, et al. (1992) contend that classification is difficult in ill-structured domains like medicine. The fact that clinical performance of the same physician may vary considerably from case to case, or even within essentially the same case at different times, is difficult to reconcile with a simple classification view. In addition, Murphy and Wright (1984) found that category distinctiveness for three pediatric categories decreased, rather than increased, with expertise level. The view that medical knowledge is highly contextualized is also at variance with categorization, or at least with categorization on basis of stable categories.

In conclusion, as diagnostic expertise can not adequately be described by models based on biomedical reasoning, prototypes, or instances, it seems not too far-fetched to assume that repeated experience with patients suffering from certain diseases results in higher level, schematic knowledge structures, that are particularly tuned towards the situations in which they are applicable, i.e., in diagnosing and treating patients. This view is endorsed by a number of researchers (Arkes \& Harkness, 1980; Gerritsma \& Smal, 1982; Johnson et al., 1981; Patel \& Frederiksen, 1984). For example, Arkes and Harkness (1980) define a cliagnosis as "a schema that summarizes the presentation of a group of symptoms." John- 
son et al. (1981) speak of disease knowledge as "... schemata or templates, which specify, for a particular disease, the set of clinical manifestations that a patient with that disease should present clinically" (p. 236). Turner (1988) emphasizes the procedural nature of disease schemas. By assuming variable slots, schema theories can relatively easily explain the incorporation of deviating information; thus, the finding that experts tend to ignore the weakest of two data-items that support two competing hypotheses (Feltovich et al., 1984), can also be interpreted as being in line with a schematic representation of disease knowledge (in an approach based on reasoning, an effort from the part of the subject would be expected to explain the presence of both items). An alternative to a schema in medicine, a causal-conditional network, is suggested by Patel, Evans, and Groen (1989). These networks, consisting of propositions connected by causal or conditionall rules, are equivalent to situation models (Van Dijk \& Kintsch, 1983). However, the Van Dijk \& Kintsch' (1983) model is especially tailored to the domain of text processing, and hence it is unclear whether it can be directly transferred to medicine. Furthermore, this model does not provide clues as to the use of nonverbal information. Hence, a schema may provide a better representation of expert medical knowledge than a causal network. In the next section, we will elaborate on a specific embodiment of the schema, especially tailored to medical diagnostic situations, and already briefly introduced in an earlier section of this chapter: The illness script.

\section{GENERAL FRAMEWORK OF THE PRESENT STUDIES: THE ILLNESS SCRIPT CONCEPT}

The concept of an illness script is an elaboration of the more general idea that medical diagnostic and clinical knowledge is organized in a kind of schemas. These schemas are disease oriented and contain information about patient background factors, complaints, signs, symptoms, diagnostic procedures, course, and treatment. They are especially tuned towards use in routine diagnostic situations, which means, according to Norman (1988), applicable in about 19 out of 20 cases. In addition, they are sensitive to variations in population frequencies and absence, presence or typical values of symptoms, and make it easily possible to predict the presence or absence of other findings, and the typical or expected course of a disease. The construct of illness scripts is especially suited to account for the phase of initial hypothesis generation in a diagnostic situation, of which the importance is, in our view not fully acknowledged by many authors, particularly those who emphasize the hypothetico-deductive nature of the diagnostic process (e.g., Barrows \& Feltovich, 1987; Elstein et al., 1978; Feltovich et al., 1984; Patel \& Frederiksen, 1984; Patel \& Groen, 1986a). Explaining hypothesis generation by "induction" (Patel \& Frederiksen, 1984) or "forward reasoning" (Patel \& Groen, 1986a) is, in our opinion, not sufficient.

However, many of these authors show awareness of the fact that knowledge organization plays a key role in hypothesis generation (cf. Elstein et al., 1978; Lesgold, 1984; Patel \& Frederiksen, 1984). "The notion of an illness script, as an effort to model such a knowledge organization, was first expanded upon by Feltovich and Barrows (1984); the expression "illness script" was coined by Clancey (1983), against the background of the MYCIN system. Early vestiges of what we now call an illness script can also be found in Johnson et al. (1981). Though "illness schema" would probably also be an appropriate term, illness script is preferred here 
because it concerns a specific elaboration of the more general notion of a schema, and also is closely related to the script idea of Schank and Abelson (1977).

An illness script is a narrative structure in which disease knowledge is represented in a way that is particularly tuned towards use in practical, climical situations. In line with the Schank and Abelson (1977) script approach, illness scripts also describe generalized events (i.c., diseases), are represented as interconnected wholes, and consist of slots that can assume certain values. Moreover, they can be activated by hearing or mentioning the script title, script actions, or even specific slots, and instantiated by providing specific values to be filled in the slots. According to Feltovich and Barrows (1984), an illness script consists of three components:

1) Enabling Conditions, i.c., the factors that influence the probability that someone gets a particular disease. Examples of Enabling Conditions are: Age, sex, physical appearance, hereditary dispositions, occupation, living environment, medical history, previous and current medication, and risk behavior. Enabling Conditions comprise a broader range of information than the contextual features as envisioned by Hassebrock and Prietula (1990) and Van Rossum et al. $(1990,1991)$, the predisposing factors described by Clancey (1983), and the non-medical and non-specific background information that Weber et al. (1993) deliberately included in or deleted from their cases.

2) The Fault, i.c., the pathophysiological disturbances in the body, couched in a biromedical model. Usually, this model may be subsumed under a diagnostic label (e.g., common cold, gout, stomach cancer, AIDS).

3) Consequences, i.c., the complaints, signs, and symptoms ${ }^{5}$ the Fault gives rise to. Consequences may be immediately visible, like jaundice or rubella spots, but may also require further investigation (e.g., assessment of serum sediments or renal casts in urine).

Though illness scripts are mainly useful in clinical situations, according to Feltovich and Barrows (1984) basic science knowledge plays an important role in the illness script concept: they state that knowledge of anatomy, biology, physiology, histology, biochemistry, and other basic sciences constrains and guides the manner in which script components can be structured together. Furthermore, Feltovich and Barrows speak of "...an integrated script that needs to be constructed for a patient..." (p. 140, italics added). "Thus, the way they conceptualize illness scripts seems to be more in line with the mental model approach (Gentner \& Stevens, 1983; Johnson-Laird, 1983), than with the script theory (Schank \& Abel. son, 1977). As already mentioned in the previous section, research performed in the past decade has shown that reasoning, and especially biomedical reasoning, is not a prominent feature of expert medical problem solving. Consequently, we opt for some modifications and elaborations of the original Feltovich and Barrows' (1984) illness scripts ${ }^{6}$.

First, at least in routine cases, an illness script will be directly retrieved from memory and immediately instantiated; there is no need to construct one for each individual patient, as Feltovich and Barrows (1984) contend. In our view, physicians" illness scripts are instantiated, not constructed, by seeing individual

\footnotetext{
5 "Signs" refers to the objectively measurable manifestations of a disease, "symptoms" to the more subjectively experienced phenomena.

6 Feltovich has also offered a new interpretation of the original illness script idea (Feltovich, Coulson, Spiro, \& Dawson-Saunders, 1992) that is called "illness script 2." Like in the intial illness script approach, biomedical knowledge remains important in clinical situations, a viaw not shared by the present author.
} 
patients wh a particular disease. As a consequence of diagnasing several patients with a specific disease, physicians will become susceptible to variability in complaints, signs and symptoms, and this variability will become part and parcel of their illness seript in the form of variable slots. Thus, an important aspect of the initial script idea is preserved in the present conceptualization of illness scripts.

Second, the role of Enabling Conditions is much more pronounced than in the Feltovich and Barrows (1984) approach. Obviously, diagnostic hypotheses that are generated in an early stage of the patient encounter can not be a consequence of peering into the patient's body, nor of ordering a decisive laboratory test. They can only be based on the information that is available in that early stage of the clinical process, $\dot{i} . c_{\text {., }}$ mainly Enabling Conditions. The finding that experienced physicians form qualitatively better initial diagnostic hypotheses (cf. Elstein et al., 1978; Hobus, 1994) underscores the role of Enabling Conditions in the diagnostic process, the more since Hobus, Boshuizen, and Schmidt (1989) showed that if only a complaint is provided, experts" diagnostic hypotheses are only marginally better than those of non-experts.

Third, the role of the Fault component is deemphasized. Clancey (1983) already recognized that a detalled account of how predisposing factors cause a disease often requires too much basic science knowledge, and is not directly relevant for diagnosis either. As already discussed, this latter finding is confirmed by subsequent research (e.g "Bashuizen \& Schmidt, 1992). In addition, for many diseases, the exact nature of the biomedical model underlying the Fault will either be only partly known (e.g., many neurological ailments, including Alzheimer's disease) or not worth knowing at all (e.g., a simple cold or minor infection). Furthermore, relative novices (second-year students) dispose of and are able to use primitive illness scripts, long before their basic knowledge concerning medical Faults has reached an acceptable level (Boshuizen et al., 1988).

Fourth, it should be emphasized that illness scripts are functional, psychological entities, rather than objective representations of a "medical reality." This is of particular importance for the Enabling Conditions: their utility derives from their ability to activate the associated illness script as a possible diagnostic hypothesis, regardless of the nature of the actual medical relationship between the Enabling Conditions and the proposed disease?. Thus, a more accurate definition of Enabling Conditions would be: Factors that are in principle avallable at the be ginining of a consultation, and that influence the probability that a specific script will be activated. Furthermore, some Enabling Conditions in illness scripts may actually be Consequences of other diseases: For example, if a patient has had previous episodes of angina pectoris, this may be an Enabling Condition for corow nary infarction, but both phenomena may of course also be viewed as Consequences of the same underlying Fault (i.c., atherosclerosis). Though we do not want to deny that "objective" descriptions of illness scripts, in line with medical standards, are possible, it is important to keep in mind that we are primarily talking about students' and physicians' mental constructs.

Fift, a fourth component, Course \& Treatment, should also be included in the illness script (cf. Hobus, 1994). Though its relevance for initial hypothesis generation may be limited, assessing the course of a disease by repeated inves-

7 As such, Enabling Conditions in the illness script approach can not be identified with enabling conditions in the sense of Cheng and Novick (1991): they use the expression "enabling conditions" to refer to circumstances that are necessary, but not sufficient for something to happen. For exmple, gravity is a necessary, but not sufficient, cause for a plane crash. 
tigation may play an important role in ultimate establishment of a diagnosis (Barrows \& Feltowich, 1987; Wilkins, 1970). Subsequent treatment selection will often be determined by an interaction between course evaluation and treatment knowledge. It may be argued that information about specific diagnostic procedures constitutes a separate component of a script, though it is also possible to view this as part of a script verification process.

Sixth, illness scripts do not appear out of the blue in physicians minds; they are acquired as a consequence of specific autobiographical memories of actual patients (cf. Anderson \& Conway, 1993) and gradual adaptation to constraints in daily practice (Schmidt, Norman, \& Boshuizen, 1990). At the roots of script formation lies the expectation that something once experienced will happen again in a similar way next time (Schank \& Abelson, 1977). Scripts are constantly elabow rated upon with each successive experience, probably, once they are formed, their characteristics may be influenced not only by additional experiences, but also by reading or hearing about them, or by experience with other, related scripts. Presently, the role of biomedical knowledge in illness script formation is still unclear, but its importance is likely to be limited (Boshuizen et al., 1988).

Routine diagnostic problem solving can be described as script activation, script selection, and script verification (Schmidt \& Norman, 1989). With increasing expertise, it will be easier to trigger an appropriate script (Lesgold, 1989); there is evidence that an increased capability to exploit Enabling Conditions in the early stages of a diagnostic situation can account for this expertise effect (Ho. bus, Schmidt, Boshuizen, \& Patel, 1987; Hofstra, Hobus, Boshuizen, \& Schmidt, 1988). Rapid script activation and instantiation may also be revealed by forward reasoning (Patel \& Groen, 1986a; Patel, Groen, \& Arocha, 1991). Apart from easy access by appropriate Enabling Conditions, scripts will also become more differentiated and enriched (Hobus et al., 1990), with an increasing sensitivity toward both highly typical instantiations and the practically relevant deviations from typical slot values. Frequently occurring instantiations of a script may be stored as prototypes or prototypical patients. In terms of medical decision theory, part of this increased script differentiation and sensitivity to actual manifestations of diseases can be described as the incorporation of base-rate probabilities into the scripted structure. Though there is ample evidence that physicians are no "real" Bayesians (Borak \& Veilleux, 1982; Elstein et al., 1978; Gerritsma \& Smal, 1982; Lusted \& Stahl, 1964), research has shown that they do incorporate base-rate probabilities into their diagnostic decisions (Christonsen-Sralanski \& Bushyhead, 1981; Garland, 1959; Kuipers, Moskowitz, \& Kassirer, 1988; Weber et al., 1993). For example, Weber et al. (1993) report that the set of diagnostic hypotheses constructed by physicians to account for a case generally includer at least one disease with a low probability, but a high degree of seriousness.

\section{EVIDENCE FOR THE COGNTTIV REALITY OF ILLNESS SCIRIPS}

Obviously, the existence of illness scripts can not be considered as given; empirical evidence should be collected to support the theory. First, it should be noted that illness scripts are not directly observable entities, but theoretical constructs, used to account for diagnostic behavior of physicians and medical students. Research is necessary to corroborate the idea that illness scripts are an appropriate way to describe expert medical (clinical) knowledge, and preferably that they can explain a number of findings better than alternative notions, like biomedical models or prototype representations. In addition, the proposed developmental 
course of illness scripts should also be buttressed by experimental findings. However, the last decade, some results have been obtained that bolster the idea that chinical medical expertise can be represented as illness scripts.

First, experts" recall suffers if a case is presented in a scrambled format i.e., random order, while novices' recall is not influenced by the order of presentation. Coughlin and Patel (1986) conclude that disturbing the standard patient presentation paradigm makes it difficult for expert physicians to use their illness frames or prototypes. The standard patient presentation paradigm, in which a case description starts with some contextual and background information about the patient, and proceeds via presentation setting, main complaint, additional complaints, signs and symptoms, findings on physical examination and laboratory data to (hypothesized) diagnosis, therapy, course, and prognosis, serves as a framework for the illness script. Furthermore, diseases have a more complex internal structure than the entities generally employed in category research: First, in illness scripts, the presence or absence of different features, and the values that can be assumed by variables, are not independent, and second, the time course inherent in a disease implies that some early available features, such as Enabling Conditions and the main complaint, are more useful in the diagnostic process than other features, such as laboratory values, even though these latter ones may actually be more diagnostic of the disease. To a certain extent, illness scripts can be viewed as high level theories about diseases, and recent conceptualizations of categories and conceptual coherence as theories (e.g., Medin, 1989) may be able to give a better account of disease knowledge than a classical categorization model, even though illness scripts can be in principle be formed and achieve coherence without an underlying explanatory theory.

Second, the finding that the proportion of concepts from medical basic sciences like anatomy, physiology, pathology, and histology in free recall protocols diminishes with increasing expertise (Schmidt, Boshuizen, \& Hobus, 1989; Boshuizen \& Schmidt, 1992) indirectly supports illness script theory. Experts use clinical knowledge, rather than medical basic knowledge, in constructing a representation of a patient problem. This finding, in cambination with the intermediate effect, argues against a mental model or "reasoning through" approach of medical diagnosis. Though medical basic knowledge might initially be the "glue" holding different parts of an illness script together, this knowledge is no longer used in routine cases if full-fledged illness scripts have developed. The existence of an intermediate effect is also an argument against an approach in which it is simply the extension of the knowledge base, or, conversely, more elaborate processing of knowledge, that is critical in accurate medical diagnosis. On the contrary, it is the structure, much more than the amount of knowledge or the extent of processing, that matters, at least at the advanced levels of expertise.

Third, medical experts are much more able to use Enabling Conditions to activate appropriate diagnostic hypotheses than less advanced subjects (Hobus, Boshuizen, \& Schmidt, 1989; Hobus et al., 1987; Hofstra et al., 1988). The ability to use sigms and symptoms, on the other hand, discriminates only marginally between subjects at different levels of expertise. Obviously, contextual cues have to be interpreted by knowledge structures to be applicable as Enabling Conditions. Experts" knowledge is better tuned (cf. Rumelhart \& Norman, 1978) - but what does "better tuned" mean in terms of the nature of knowledge structures? While Feltovich and Barrows (1984) opt for better development of basic science knowledge, Schmidt, Boshuizen and Hobus (1988) argue that experts' disease representations have particular script-like properties, whereas inexperienced subjects have symptom-oriented knowledge structures. This latter hypothesis was corrob- 
orated by the finding that expert, but not inexpert, physicians, described prototypical patients suffering from specific diseases, relatively more in terms of Enabling Conditions than in terms of Consequences. Furthermore, physicians sometimes spontaneously indicate that their diagnostic performance may suffer from a lack of contextual information (Gerritsma \& Smal, 1982). Finally, Boshuizen et al. (1988) conclude that subjects at all levels of medical expertise seem to apply "illness script like" structures, even at those levels at which medical basic knowledge is still lacking.

Consequently, in our view, some circumstantial evidence is already avail able to suggest that the illness script is a viable and fruitful way to conceptualize the representation of disease knowledge in physicians, experts as wel] as nonexperts. However, two important topics have to be dealt with to establish more firmly the illness script concept. The first one is the development of illness scripts, and particularly the role of Enabling Conditions in this development. Some relevant work on this topic has already been performed by Hobus et al. $(1987,1990)$. The results of these studies point towards a possible lag in the development of two main illness script components, the Enabling Conditions and the Consequences, with the Consequences presumably having an edge on the Enabling Conditions. Nevertheless, the role of Enabling Conditions in the knowledge structures of expert physicians and less experienced subjects is still relatively unknown. The second topic concerns the scripted aspect of illness scripts. As already mentioned, in the years following the publication of the Schank and Abelson (1977) work, a large number of script studies have been performed. These studies have revealed some salient memory aspects of scripts, particularly with respect to the difference in memory performance for typical and atypical script related information. If it can be demonstrated that similar memory phenomena hold for illness scripts, this would provide additional support for their psychological utility.

Therefore, four studies were performed to investigate these issues in order to buttress the illness script notion. Two of these studies focused mainly on the differential role of Enabling Conditions and Consequences in disease knowledge structures; in the other two studies, we tried to zero in on the scripted aspects of illness scripts. In all four studies, developmental aspects of illness scripts were investigated; thus, at least two different levels of expertise were included in each study.

\section{OVERVIEW OF THE PRESENT STUDIES}

In the study to be described in Chapter 2 , development and structure of illness scripts was studied by providing fourth-year students, sixthth-year students, and experienced family physicians with a so-called "scrambled case," a set of 48 case statements from which they had to reconstruct a plausible case description. With respect to the hidden disease, relevant as well as irrelevant and contradictory statements were included, for both the Enabling Conditions and Consequences parts of the case. The study aimed at investigating whether a relationship will be found between level of experience and the number or nature of the Enabling Conditions included in the reconstructed case.

In Chapter 3, a study will be described which focused on active generation of medical case information, instead of "simply" recognizing it, as was the case in the Chapter 2 study. Furthermore, one additional level of expertise was included: interns, i.e., graduated physicians who were in training as family physicians. In 
this study, subjects at different expertise levels were asked, for a number of different diseases, to describe either the prototypical patient or the clinical picture associated to those diseases. Purpose of this instruction was to investigate whether more experienced subjects would volunteer more Enabling Conditions when providing disease information, and whether the type of instruction (i.e., focused on Enabling Conditions, as in the instruction to deseribe a prototypical patient, or focused on Consequences, as in the instruction to describe the clinical picture) influenced the amount of Enabling Conditions produced. It was hypothesized that generally, more experienced subjects would volunteer more Enabling Conditions, but that they would be less influenced by the particular type of instruction employed, as Enabling Conditions will be more integrated in their disease knowledge.

The nature of the study that will be described in Chapter 4 was completely different. The basic assumption in this study was that if illness scripts are gradually formed and established, experienced subjects will process case information quicker than less experienced subjects, and information that is completely in line with what might be expected given a particular disease, will be processed quicker than information that is at variance with it. Thus, in this study, case descriptions differing in degree of typicality were presented to subjects at two different levels of expertise, i.c., sixth-year students and experienced family physicians, and the time needed to process the case was be recorded. In addition, subjective disease probability estimates were taken, and analyzed in order to find out whether cases differing in typicality also received different probability estimates. We also investigated the respective influence of typicality of Enabling Conditions and Consequences on both dependent variables.

Chapter 5 presents a study that aimed at investigating the similarities and differences between "reall world" scripts and illness scripts. On basis of a number of recent, script-related studies, the results of an illness script recognition study were predicted. It was hypothesized that experienced subjects will show high false-alarm rates and slow reaction times if they have to decide whether a prototypical information item had indeed been presented during case presentation, whereas atypical or case inconsistent items will be recognized with much more accuracy and larger speed. For less experienced subjects, with less established illness scripts, these results will be much less outspoken.

Finally, in Chapter 6 , an effort will be made to integrate recent research findings and theories about problem solving and expertise development with the present results. Implications for both theory and practice, in case medical education, will be provided.

The problem on page 5 is adopted from the Dutch version of the "King William's Test Paper," published by "de "Tijd", December 1987. The inventor in question is Rudolf Diesel (1858-1913). 


\section{CHAPTER 2}

\section{THE DEVELOPMENT OF ILUNESS SCRIPTS: RESULTS OF A "SCRAMBLED CASE" RECONSTRUCTION STUDY}

\section{INTRODUCTION}

As indicated in the introductory chapter, illness scripts are hypothesized mental structures that are used by physicians to deal with clinical information in a diagnostic setting; in fact, the clinical knowledge of physicians is supposed to be organized as a large set of illness scripts (Schmidt et al., 1992; Schmidt \& Norman, 1989). In experienced physicians, these illness scripts are full-fledged and contain a wealth of clinical information, including contextual information in the form of Enabling Conditions. In students and inexperienced physicians, on the other hand, their development has not yet been completed, and particularly knowledge of the Enabling Conditions component is still lacking. For example, Hobus et al. (1989) found that experienced physicians' descriptions of idiosyncratic prototypical patients contained proportionally more Enabling Conditions than inexperienced physicians' descriptions. In addition, Hobus et al. (1987) report higher recall of relevant contextual information of a case for experts than for novice physicians. Therefore, it was concluded that experienced physicians' illness scripts are enriched with knowledge of Enabling Conditions, while novices' illness scripts are predominantly "symptom oriented," i.e., contain relatively much knowledge about the Consequences component of illness scripts.

However, Hobus et al. (1989) used a free production task. Hence, it can not be excluded that differences in task interpretation are responsible, to a certain extent, for the observed expertise effects. Generally, no reliable conclusion can be drawn from the absence of information in free production protocols: Subjects may lack the unstated knowledge, but they may also be reluctant to mention it, for example, because they are unsure as to whether it is correct, or whether the information is worth mentioning. Thus, it could be the case that the less experienced subjects in the Hobus et al. (1989) task mentioned less Enabling Conditions because they considered this information not a part of their image of a prototypical patient, or were not sure whether it would be correct. Furthermore, Hobus et al. (1989) analyzed their data with respect to the proportion of Enabling Conditions mentioned by the two expertise groups. Though this procedure is entirely justified if there are between-group differences in general verbosity, it can not be denied that the differences between novice physicians and experienced general practitioners as far as the actual number of Enabling Conditions mentioned were less than impressive: novices produced on the average 2.61 Enabling Conditions per disease, experts 3.07. Finally, though the least experienced subjects were called "novices" in the Hobus et al. (1989) study, it should be emphasized that they were novice physicians, hence already one stage beyond advanced students, with about two years experience in clinical settings: They had finished or almost finished their clerkships. Consequently, no information was gathered concerning the initial stages of illness script development.

Therefore, the aim of the study to be described in this chapter was twofold. First, it might be hypothesized that less experienced subjects do have knowledge of the importance of contextual factors as Enabling Conditions, but, for whatever 
reasons, are not as apt as experienced subjects to verbalize it in a free production task. If this were the case, then another research paradigm, based on recognition rather than recall, would probably reveal that inexperienced subjects do not differ in their knowledge of Enabling Conditions. In other words, if inexperienced subjects are less able than experts to recognize the importance of Enabling Conditions, this is probably due to a lack of the pertinent knowledge. If, on the other hand, less experienced subjects are about equally well able to recognize this kind of information as experienced family physicians, but are less inclined to produce it under recall conditions, than the observed differences in the Hobus et al. (1989) study might be accounted for by different task interpretations, or by different accessibility of the knowledge, rather than by its mere absence or presence. Second, in order to study in more detail the development of these knowledge structures, a third level of experience, i.c., fourth-year students, were included in the present study. As these students have attended 3.5 to four years of formal education in medical basic science and medical skills, but have hardly any experience with actual patients, they may be properly labeled "advanced preclinical students."

For the present purposes, a specific type of task was selected as a vehicle for investigating developmental differences in illness script knowledge in general and knowledge of Enabling Conditions in particular: A scrambled case reconstruction task. The "scrambled case" approach is especially suited to investigate complex medical knowledge structures. The core idea behind the method is that complete case information is presented to the subject, but not organized according to the standard patient presentation order. Generally, a case description is not a random list of medical facts and findings, but proceeds in a specific, "story like" sequence. Such a description starts with the name, sex, age, occupation, and civil status of the patient. Next, the medical history and the current use of medicine is mentioned, followed by the situation (e.g., consultation hour or emergency), and the main complaint. Then, information about possible additional complaints, signs, symptoms, findings on inspection, and physical examination is given. Finally, the relevant laboratory values and results of additional diagnostic procedures are provided, supplemented by information about treatment and prognosis. Of course, information on all these topics will not be present in every case, but almost always the main slots (age and sex, situation, complaint, some symptoms and findings upon examination) will be filled. This general patient presentation order parallels the frame structure of the illness script (Schmidt ot al, 1990), and it may also function as a retrieval structure for case recall (cf. Ericsson \& Strazewski, 1989). The assumption that the standard patient presentation schema, as a narrative structure, is similar to the general frame of an illness script was supported by Coughlin and Patel (1987), who found that removing this structure, i.e. presenting patient information in a random sequence, impeded experts" performance (diagnostic accuracy as well as recall) to a much larger extent than novices' performance.

In a scrambled case task, the subject is confronted with pieces of information from a case, presented in a random order. He has to reconstruct the case from these shattered pieces of information. If an illness seript is used to accomplish such a task, it may be expected that the final solution reflects to a large extent this illness script. In the present study, the separate pieces of information were not presented sequentially, but all at once, printed on small cards. Subjects" task was to reconstruct a case from the presented information by selecting those items that together constituted the case. This procedure was chosen to minimize memory load, and to make it a real recognition task. In addition to the information items that logether formed a case, a relatively large amount of distracting 
information, which could be either irrelevant or inconsistent with the case, was also provided.

An advantage of this research paradigm is also that, unlike free production studies, no categorization or interpretation problems arise in analyzing the data: The experimental materials are constructed and can be categorized in advance. Furthermore, the task is relatively easy to administer.

\section{METHOD}

\section{Subjects}

Subjects were 21 fourth-year students, 20 sixth-year students and 21 experienced family physicians. The fourth-year students had virtually mo clinical experience, the sixth-year students had followed clerkships for at least 18 months, and the family physicians had on the average 15.2 years experience in a practical setting (range: 5.75 to 41 years).

\section{Material}

The material consisted of a set of 48 small cards, each containing a statement about a patient. Appendix 2.A shows the 48 statements. Statements concerned Enabling Conditions or Consequences; one connective statement, devoid of medical content, was included to enable subjects to construct a narrative case description ${ }^{8}$. Sixteen of the remaining 47 statements were Enabling Conditions.

A specific subset of three Enabling Conditions and five Consequences formed a reasonably good, but minimal description of a patient with glandular fever (Pfeiffer's disease). With the qualification that physicians might disagree about the actual status of some of the information items, of the remaining 39 statements, thirteen were peripheral but in line with the dissease, ten were irrelevant, and sixteen were inconsistent with it. Though a few alternative diseases could in principle be used to construct a case on basis of this material (e.g., a common cold, Hodgkin's disease, Hepatitis A), glandular fever was quite conspicuous, and none of the alternative diseases would match the information in the set of statements that well.

\section{Procedure}

The set of cards was presented to the subjects as a small pile, with the individual cards in reverse alphabetic order, according to the first character of the statement. Subjects were instructed to construct a plausible case description by selecting a subset of the set of cards. They were told that they had to discover the true nature of the hidden disease by themselves, and that, in case they were of the opinion that there was more than one possibility, they had to construct only one case. Subjects were allowed to include as many or as few cards as they wished in the case, as long as it remained, in their view, a plausible patient description.

Subjects were recommended to spread the cards in front of them on the table in order to get a proper impression of the content of the material. After they had made a selection, they were asked to show it to the experimenter. Next they were requested to select an additional four or five cards from the remaining unselected

8 The text of this statement was: "Physical examination revealled:" 
cards, in order to expand the case, without changing its medical content. Finally, they were asked to remove from the original selection four or five cards that could be dispensed with without changing the meaning of the case. Thus, in fact three case descriptions were collected for each subject: a core case (the original selection with four or five cards removed), a basic level case (the original selection) and a case extension (four or five additional cards). Subjects were not required to present the cards in the exact order, though most of them spontaneously did so; neither were they asked to explicitly provide a diagnosis. No time constraints were imposed; subjects were essentially free to spend as much time as they wanted.

\section{Analysis}

The total number of cards in the original selection and the number of Enabling Conditions and Consequences cards were computed. The card with the connecting statement was excluded from the analysis, as it belonged neither to the Enabling Conditions, nor to the Consequences. Thus, if proportions of total number of statements are used in the results, these are based on the remaining 47 statements.

As the Enabling Conditions statements in the present task were directly available to the subjects, and did not have to be retrieved from memory, it might be hypothesized that especially subjects at lower expertise levels were induced to select Enabling Conditions as a consequence of the task design, but that they did not assign that much importance to these statements. However, if this were the case, family physicians would be more inclined than sixth-year students to incorporate Enabling Conditions into the "core case." Or, conversely, fourth-year students might be more inclined to drop Enabling Conditions if their task was to reduce the number of case statements. In order to investigate this possibility, we computed a composite Enabling Condition score, in which Enabling Conditions in the core case, the basic level case, and the case extension were assigned weights of 3,2 , and 1, respectively. Thus, Enabling Conditions which remained in the selection even when statements had to be removed, received a higher weight than Enabling Conditions that were only included after the experimenter insisted on extending the case; the composite Enabling Conditions score for each subject was simply the sum of the weighted Enabling Conditions. Reluctance to remove Enabling Conditions would result in a relatively high composite score, whereas the inclusion of Enabling Conditions merely as a consequence of a request to extend the reconstructed case would lead to a relatively low composite score.

To account for between-subject differences in size of the selected subsets, subjects were also compared as to the relative proportion of Enabling Conditions and Consequences selected: Values were also expressed as a percentage of the complete set of 47 cards. Analyses of variance were performed to investigate differences between the three expertise levels.

Furthermore, some more fine-grained analyses on the present data were performed. First, for each statement, it was counted how many subjects had included it in their original case reconstruction, and this number was expressed as the percentage of the total number of times it was selected by subjects at that particular expertise level. Subsequently, Pearson correlation coefficients were computed between the number of subjects at the three expertise levels that selected a particular statement in their case reconstructions. Finally, $\chi^{2}(2, N=62)$ analyses 
were performed for each individual statement to investigate whether a differential selection preference existed at the three expertise levels included in the study.

\section{RESULTS}

The main results are depicted in Table 2.1. One-way analysis of variance rem vealed no significant effect from expertise level on total number of statements selected $(F(2,61)=2.111, p=.1301)$. Fourth-year students, sixth-year students and family physicians selected on the average $12.24,14.15$, and 12.19 cards, respectively. Thus, the present results showed neither an increase nor a decrease in general case extensiveness with expertise level.

Table 2.1.

Results of the scrambled case reconstruction task.

\begin{tabular}{cccc} 
Dependent measures & $\begin{array}{c}\text { Fourth-year } \\
\text { students }\end{array}$ & $\begin{array}{c}\text { Sixth-year } \\
\text { students }\end{array}$ & $\begin{array}{c}\text { Family } \\
\text { physicians }\end{array}$ \\
\hline
\end{tabular}

Average number of

statements selected

12.24

14.15

12.19

Average number of Enabling

Conditions selected

Average number of

Consequences selected

9.80

Enabling Conditions as percentage of number of statements selected

32.40

Average Composite Enabling Conditions score

Number of Enabling Conditions in core case

Table 2.1. shows that more experienced subjects were not inclined to select more Enabling Conditions than less experienced subjects; analysis of variance revealed no significant main effect of level of expertise. At all levels of experience, about four Enabling Conditions were included in the selected subset of cards. Thus, the hypothesis that experienced physicians would select more Enabling Conditions than students, could not be confirmed.

Similarly, no relationship was found between expertise level and number of Consequences statements selected. As the present case consisted of only Enabling Conditions and Consequences, analysis of number of Consequences does not add anything to the two previous analyses.

Converting the raw data on Enabling Conditions into percentages of the total number of statements selected also does not change this picture. About one out of 
every three statements selected was an Enabling Condition, for all three expertise levels alike. The apparent tendency of family physicians to select a little more Enabling Conditions fell far short of significance, $F(2,61)=1.409, p=.2526$.

Table 2.2 ,

Most and least often selected statements, and percentage of subjects that included these statements in their case reconstruction.

Statements most often included in the reconstructed case:

Type $^{\mathrm{a}} \% \mathrm{sel}^{\mathrm{b}}$ Statement Text

$\begin{array}{lll}\text { EC } & 67.74 & \text { Rutger, 17 years old, 5th grade HAVO } \\ \text { EC } & 61.29 & \text { Shows up during office hours with the complaint of: } \\ \text { Con } & 53.23 & \text { Neither liver nor spleen are enlarged } \\ \text { Con } & 50.00 & \text { "Lately, I feel so spiritless and languid" } \\ \text { EC } & 48.39 & \text { Oldest sister (21) has had glandular fever three months ago } \\ \text { Con } & 46.77 & \text { Bilateral enlargement of glands in neck and arm-pits } \\ \text { Con } & 45.16 & \text { A few days ago, he has had a terrible throat-ache, but it has } \\ & & \text { subsided } \\ \text { EC } & 43.55 & \text { At the age of eight, tonsillectomy was performed } \\ \text { EC } & 43.55 & \text { Middle child from a family of three children } \\ \text { Con } & 40.32 & \text { However, he did not vomit }\end{array}$

Statements least often included in the reconstructed case:

Type $^{\mathrm{a}} \%$ sel. $^{\mathrm{b}}$ Statement Text

EC 9.67 At his work, many employees are struck by the flu

Con 9.67 The patient is in bed, and perspires heavily

Con 9.67 Palpation reveals no enlarged liver

EC $\quad 8.07$ Family history of diabetes mellitus

Con $\quad 6.45$ "At work, I have difficulty to sustain my attention"

EC 4.84 A month ago, his youngest son (12) was diagnosed as having Hepatitis A

EC $\quad 3.23$ Man, 71 years, retired since 8 years

EC $\quad 3.23$ His wife calls: "Would you please come and have a look at my husband"

\footnotetext{
"Statement type, $\mathrm{EC}=$ Enabling Condition, $\mathrm{Con}=$ Consequence

b Percentage of subjects that selected the particular statement $(\mathrm{N}=62)$

- Type of secondary school in the Netherlands
} 
In addition, it was tested whether subjects from different expertise groups, though generally selecting an approximately equal number of Enabling Conditilons, might perhaps show a differential tendency to adhere to the Enabling Conditions they had initially selected. Thus, we also performed an analysis of variance on the composite Enabling Conditions score. Once again, no significant differences were found, indicating that subjects at all three expertise levels were about equally inclined to retain Enabling Conditions in the selected set, even if requested to constrain the size of the reconstructed case. Table 2.1. also shows that the core case, i.e., the set of statements preserved after the case had been "stripped," contained an almost equal number of Enabling Conditions for all expertise levels, a finding that is of course completely in line with the previous one.

It might be argued that it is the nature of the Enabling Conditions selected, rather than their actual number, that distinguishes experienced from non-experienced subjects. In order to test this possibility, we investigated whether there were between-group differences in the number of times certain statements were selected. First, it should be pointed out that a large majority of the subjects, at all expertise levels, constructed a "glandular fever like" case from the available statements. Though we did not explicitly ask subjects to prowide a diagnosis, we might conclude this from the number of times statements which were in line with this diagnosis were included in the reconstructed case, compared to statements that were contradictory to it. Table 2.2 shows the statements selected by either $40 \%$ or more of the subjects, and those selected by less than $10 \%$ of the subjects, all three expertise levels included. Appendix 2.A shows the relevant data for all statements. All statements selected by $45 \%$ or more of the subjects were in line with the diagnosis "Pfeiffer's disease," while all statements selected by $10 \%$ or less of the subjects were indicative of another type of illness (e.g.s items like older age, heavy perspiration, and colleagues having the flu, are more or less incompatible with glandular fever).

To test for differences in statements selected between expertise levels, Pearson correlation coefficients were computed between the number of times each statement was selected at the three expertise levels. Thus, in order to perform this analysis we substituted statements for subjects in the usual analysis and calculated three variables for each statement: For each expertise level, the number of times it was included in the original case reconstruction. To control for the small differences in number of subjects in the three groups, the percentage of subjects at each expertise level that selected a specific statement was computed for each individual item. Subsequently, Pearson correlations for this dependent variable were computed for each combination of two expertise levels. A high correlation indicates that if a particular statement was often selected by one of the two compared expertise groups, it was also often selected by the other group. Or, in other words, the higher the correlation coefficient, the more the two groups re sembled each other in their selections, and thus in their case reconstructions. The values of the correlations were .79 between fourth-year students and sixthyear students, 74 between fourth-year students and family physicians, and .65 between sixth-year students and familly physicians. Thus, a reasonable agreement between the three expertise levels was found as far as their choice of statements is concerned, but the correlations did not show a lagged pattern. Consequently, there is no evidence that adjacent levels of expertise resemble each other more than the two extreme levels.

Finally, $\chi^{2}(2, N=62)$ analyses for individual statements were performed to investigate whether some statements were perhaps consistently more often se- 
lected by subjects at higher or lower level of expertise. Only one of these $\chi^{2}$-analyses was significant at the .001 level ${ }^{9}$. Unfortunately, this statement showed an intermediate effect, and not a straightforward, easily interpretable relationship between expertise level and probability of being selected. The statement read: "The preceding days, he has scarcely eaten anything," and it was selected by $33.3 \%$ ( 7 out of 21 ) of the fourth-year students, by $70 \%$ (14 out of 20 ) of the sixth-year students, and by only $14.3 \%$ ( 3 out of 21 ) family physicians, $\chi^{2}(2, N=62)=13.789$, $\mathrm{p}=.001$. The only Enabling Condition statement that showed something remotely reminiscent of a linear relationship with expertise level read: "Eldest sister (21) has had glandular fever three months ago," which was selected by $33.3 \%$ of fourth-year students, $50 \%$ of sixth-year students, and $61.9 \%$ of family physicians, $\chi^{2}(2, N=62)=3.463, p=.177$.

\section{DISCUSSION}

From the results, it can be concluded that the scrambled case reconstruction task exploited in this study as a tool for revealing expert novice differences failed to show these purported differences, even between subjects at rather disparate levels of expertise. Several interpretations of these non-significant results are possible. First, an explanation that can not be definitely excluded is that the present findings are not representative of the actual situation, for example, because we happened to succeed in choosing just one of the few diseases that novices are equally experienced with as experts. Though we immediately agree that one case is not a sufficiently stable foundation to test a theory of expertise development in a complex domain, it seems likely that similar results would be obtained from other diseases. The illness of glandular fever was chosen because a number of rather aspecific complaints, signs and symptoms are associated to it; Consequences that might also be present in a host of other diseases. In addition, Enabling Conditions as well as Consequences of some of these alternative diseases were included in the material. On the other hand, as Table 2.2 implicitly reveals, reconstruction of a case of Pfeiffer's disease was, for a majority of subjects at all three expertise levels, rather clear-cut. But even if every subject should discover the nature of the hidden case, the material was constructed in a way to enable expert-novice differences to seep through; for example, it still would be possible that the importance of Enabling Conditions was acknowledged to a larger extent by subjects at more advanced expertise level, as the illness script theory would predict. The data, however, do not support this explanation: It appears that preclinical students" ability to select a case description from a pool of noisy disease information does not differ from experts' ability to perform such a task. Subjects at the three expertise levels included in the study constructed a highly similar case, and inter-individual differences completely overshadowed the differences between expertise groups, should these exist. In addition, less experienced subjects were not less inclined to include contextual information, in the form of Enabling Conditions, into the reconstructed case. Hence, it seems reasonable to conclude that the present scrambled case reconstruction task is not sensitive enough to reveal differences between novices and experts - unless one should want to conclude from the results that these differences in fact are non-existent. To a

9 As 48 of these $\chi^{2}$-analyses were performed, we had to adopt a much more rigid significance level than the ususal 05 . 
certain extent, the conclusion that the present task is not sufficiently sensitive is corroborated by the finding that an important Enabling Condition, i.e., the presence of Pfeiffer's disease in the sister of the patient described in the case, was selected by one out of every three fourth-year students, half of the sixth-year students, and almost two-third of the family physicians. This finding is completely in line with the expectations; unfortunately, it failed to reach significance.

Generally, as subjects do not need to generate information by themselves in this type of task, but only have to select it and put it in the proper order, it can be considered much less demanding than tasks in which subjects do have to recall or produce information, let alone tasks in which they have to diagnose or explain a case. On the other hand, it seems likely that a scrambled case reconstruction paradigm does not reveal differences between subjects at different levels of medical expertise, either because it is not sensitive enough, or because these differences should be located in other aspects of subjects' knowledge structures than in the ability to recognize and reconstruct a case of a relatively familiar disease. Though the present task shares this failure to yield significant differences between subjects at different levels of expertise with a number of other studies (e.g., Barrows, Feightner, Neufeld, \& Norman, 1978; Elstein et al., 1978; Gale \& Marsden, 1983; Wortman, 1966), an important difference between these studies and the present one is that the former studies focused mostly on diagnostic process variables, while the scrambled case approach was especially designed to tap medical knowledge more directly. Consequently, in addition to previous conclusions that experts and novices do not differ on several clinical process variables, the present study supports the hypothesis that it is not so much the mere presence or absence of certain content knowledge that accounts for expert-novice differences, but that it is particularly the way in which this knowledge is structured and used that forms the source of expert-novice differences. This conclusion is in line with the results of Hobus et al. (1989).

Maybe, a more open task like the one employed by Hobus et al. (1989) can be extended to obviate some of the methodological risks inherent in this approach. In the next chapter, a study will be described that partly replicates, and partily extends this Hobus et al. (1989) study. In this study, we shall return to a free production task under the assumption that it is a more sensitive tool to reveal expertnovice knowledge differences. 



\section{CHAPTER 3}

\section{THE RELATIONSHIP BETWEEN MEDICAL EXPERTISE AND THE DEVELOPMENT OF ILLNESS SCRIPTS: Evidence from an interview study}

\section{INTRODUCTION}

Recent research in the area of medical expertise has revealed some findings that can be considered the starting point of the study to be described in the present chapter. First, the source of the differences between medical experts and nonexperts should be located in the quality of the knowledge structures, and not, for example, in general problem solving abilities or better execution of prescribed diagnostic procedures (cf. Boshuizen \& Schmidt, 1992; Elstein et al., 1978; Feltovich et al., 1992; Patel \& Groen, 1986a). Second, in medical diagnostic situations, these differences in knowledge structure exert their influence from the very first moment of the consultation: Experts have what might be called a "head start" (Barrows et al., 1982; Elstein et al., 1978; Hobus et al., 1987) as far as diagnostic accuracy is concerned. For example, Elstein et al. (1978) report that in about $90 \%$ of diagnostic consultations, a first diagnostic hypothesis is activated as soon as the patient has phrased his main complaint, and subsequent research has revealed a positive relationship between the quality of these early hypotheses and the level of expertise of the subject (Elstein et al., 1978; Johnson et al., 1981; Neufeld et al., 1981). Third, however, as the research reported in the previous chapter attests, there are also aspects of medical knowledge structures that do not differentiate between experts and novices: performances of subjects at three levels of expertise (i.c., fourth-year students, sixth-year students, and experienced physicians) on a scrambled case reconstruction task were essentially the same. The ability to recognize and select disease relevant information from "noise" in a database appears to be equal for advanced preclinical students and family physicians. Especially the sensitivity of more experienced subjects for contextual information, a salient result in the Hobus et al. $(1987,1988)$ studies, could not be confirmed.

However, the fact that less experienced subjects were able to recognize the important features of a patient suffering from a particular disease, does of course not necessarily imply that they are able to use this information in a diagnostic setting. According to the illness script theory, this latter ability requires the integration of knowledge concerning the importance of contextual information about patients into the Enabling Conditions component of an illness script (cf. Schmidt \& Boshuizen, 1993a). Medical diagnosis presumably requires much more elaborate and finely tuned (cf. Rumelhart \& Norman, 1978) knowledge structures than those that can be considered responsible for the similar performance of experts and non-experts in a scrambled case reconstruction task. Experts are better diagnosticians than novices because their knowledge is organized and structured in full-fledged illness script with integrated Enabling Conditions; these structures make quick activation and easy access of this knowledge in actual diagnostic situations possible.

Illness script theory states that medical knowledge changes during the development of medical expertise. In the preclinical phase, detailed biomedical knowledge is gathered and an elaborate network of factual medical knowledge is 
constructed. In this phase, illness scripts are merely theoretically based mental constructs, in which contextual factors, signs, symptoms, and complaints are held together by a network of elaborate biomedical explanations. By experience in practical settings, for example during the clerkship phase, illness scripts gradually become more full-fledged and integrated, and knowledge about the importance of patient background factors becomes more prominent. Simultaneously, the role of overt biomedical knowledge in clinical reasoning decreases, because, at least for diseases with which the student or beginning physician has a certain amount of practical experience, extersive biomedical reasoning to interconnect the different features that characterize a specific disease, is no longer necessary. Furthermore, a salient feature of illness script development is that knowledge of the complaints, signs and symptoms associated with a disease (i.e., the Consequences component of the illness script) goes ahead of knowledge of the Enabling Conditions, an may even reach a certain maturity in a relatively early stage of the development of medical expertise. Probably, it takes more practical experience to integrate Enabling Conditions into an illness-script structure than to add Consequences to it. Thus, the acquisition of a knowledge base of sophisticated and full-fledged illness-scripts requires several years of medical study, supplemented by a large number of patient-encounters in practical settings.

Thus, illness script theory does not state that subjects who are less experienced in the medical domain lack all of the relevant knowledge, but rather that this knowledge, if present, is not integrated in fully developed scripted structures, but consists of collections of relatively scattered fragments, held together by embryonic illness scripts. Consequently, if such a structure is externally presented, like in the scrambled case reconstruction task described in the previous chapter, differences in performance between subjects at several expertise levels might decrease, or even vanish completely. However, if subjects have to provide a scripted structure by themselves, differences due to level of experience might become manifest.

Such was the case in an experiment conducted by Hobus et al. (1989), of which the present experiment is an elaboration and extension. Hobus et al. (1989) presented subjects at two different levels of expertise, i.c. sixth-year students (who were about to graduate or had recently graduated) and experienced family physicians, with a number of complaint-diagnosis pairs. The subjects' task was to describe a prototypical 10 patient with that diagnosis (disease), who would present him- or herself with the complaint in question. For example, one of the instructions was: "Can you describe a prototypical patient suffering from herpes zoster, who presents himself with the complaint of a terrible pain in a specific, wellcircumscribed region on the chest." "The hypothesis was that expertise based differences would become especially manifest on the Enabling Conditions component of the illness script. In other words, experienced family physicians would produce descriptions of prototypical patients with a large proportion of Enabling Conditions included, while the descriptions of sixth-year students were expected to be more centered around the Consequences component.

10 In the Hobus et al. (1989) experiment, like in the one to be presented in this chapter, subjects were asked to describe a prototypical patient; though intended was a "typical" patient, the expression "prototypical" was used for two reasons: first, because a strong association with the prototypicality theory of categorization was assumed to hold, and second, the word "typical" has a somewhat different flavour in Duteh, compared to English: in Dutch, a "typical patient" 
This hypothesis was corroborated: The descriptions of sixth-year students contained about 35\% Enabling Conditions and 59\% Consequences, while the experienced physicians provided $51 \%$ Enabling Conditions and $41 \%$ Consequences. As expected, the amount of biomedical information given was very limited for both levels of expertise (about $6 \%$ for the advanced students and $8 \%$ for the family physicians). However, if also the number of so-called "nonmodel information units," i.e., relevant statements that could not be categorized into one of the three illness script categories, like information about the expected course or the per. tinent therapy, was included in the analysis, the picture changed somewhat: Expert physicians turned out to mention proportionally twice as many of these non-model units compared to inexperienced physicians ( $28 \%$ vs. $14 \%$ ), while the latter subjects were more prolific as far as the Consequences were concerned (experts 30\%, 6th-year students 51\%). Differences between the two expertise levels on the relative contribution of Enabling Conditions were less impressive (experts $37 \%$, non-experts $30 \%$ ).

Therefore, it was decided to replicate the Hobus et al. (1989) experiment with some modifications and extensions, in order to investigate more thoroughly the presumed scripted knowledge structures of subjects at different levels of medical experience. The general hypothesis was that if the illness script theory is a viable approach, then the descriptions of diseases by subjects at different levels of exper"tise will reflect a development towards integrated knowledge structures that gradually become enriched with Enabling Conditions. Hence, if requested to tell something about a disease, expert physicians will tend to activate the illnessscript of that particular disease and will generate relatively many Enabling Conditions and little biomedical knowledge (i.c., Fault-elements). Preclinical students, on the other hand, will give descriptions more in accordance with medical textbook structures and thus will be more apt to produce biomedical knowledge, while they will provide little information about Enabling Conditions. An intermediate position will be taken by advanced students and relatively inexperienced physicians: As their illmess-scripts are relatively well-developed with respect to the Consequences component, symptoms, signs and complaints will play a prominent role in their descriptions of prototypical patients, while the contribution of both biomedical information and Enabling Conditions will be smaller.

Another question, also addressed in the present study, concerns the probable influence of the nature of the probe. In the Hobus et al. (1989) study, subjects were always asked to describe a prototypical patient with a particular disease. However, such a procedure may have biased the stories of the less experienced subjects towards those of more experienced: It might be the case that asking someone to describe a prototypical patient tunes the description towards the inclusion of Enabling Conditions, while a salient difference between experienced and less experienced subjects precisely might be that the former ones always involke the image of a patient when asked to tell something about a disease, while the latter ones are ordinarily not inchined to activate patient-related information, unless they are explicitly encouraged to do so. Thus, whereas half of the subjects indeed were asked to describe a prototypical patient, the other half was presented with another probe: they had to describe the clinical picture associated with each of the diseases. It was assumed that such a probe would activate the same signs and symptoms for a particular disease as the instruction to describe a prototypical patient, but would to a lesser extent focus subjects' attention on the contextual factors. For example, age, sex, and medical history would be part and parcel of the description of a prototypical patient, but might not be included in the descrip- 
tion of the clinical picture of a disease. The expectation was that experienced subjects will always include some Enabling Conditions into their descriptions, even if asked to describe the clinical picture of a disease: Their knowledge of contextual factors, signs, symptoms and complaints is integrated in illness script structures. Less experienced subjects, on the other hand, may possess the relevant knowledge of Enabling Conditions, but as they lack mature, integrated illness scripts, will be less inclined to activate, and consequently mention, this kind of information if probed to describe the clinical picture of a disease. However, if explicitly asked to describe a prototypical patient with a particular disease, they will be more inclined to activate patient-related information.

The Hobus et al. (1989) study was also extended by including subjects at four, instead of two, different levels of expertise: fourth-year students, who had virtually no practical clinical experience; sixth-year students, who had finished their clerkships or were about to do so; interns, i.e., postgraduate medical students in training as family physicians 11 ; and experienced family physicians.

Furthermore, in contrast to the Hobus et al. (1989) study, no information about the presenting complaint was provided, in neither of the conditions. Though this may have decreased the comparability between the two studies, it should be emphasized that, according to illness script theory, the presenting complaint is an important Consequence of the disease, which we did not want to "give away." Moreover, if the presenting complaint was given in both conditions, it would have decreased the difference between them and hence, probably the expected differences in effect would also diminish. Consequently, compared to the Hobus et al. (1989) study, the present experiment might be expected to yield a larger contribution of Consequences in the descriptions produced by the subjects, because it is likely that subjects will mention the usual presenting complaint(s) of a disease as part of the Consequences component of their illness scripts.

Another aspect was also added to the Hobus et al. (1989) paradigm. It might be expected that subjects' descriptions of clinical pictures or prototypical patients are influenced by their actual experience with the diseases they had to provide information about. Consequently, by means of a simple inventory, the amount of practical experience subjects had with the diseases used in the study was investigated. It was expected that this inventory, as opposed to the rather crude measure of general level of expertise, would yield data that could enable a more finegrained analysis of the influence of actual experience, on the information produced by subjects being instructed to describe either a clinical picture or a prototypical patient.

In summary, the following hypotheses were tested:

1) If Enabling Conditions become increasingly integrated in illness script structures, the number and/or proportion of Enabling Conditions produced in a free production task might be expected to increase monotonically with expertise level.

2) The contribution of biomedical, or Fault-related, information in the free production protocols will decline with increasing expertise level, because physicians tend not to use biomedical knowledge in a direct fashion in diagnostic situations.

3) If experts' illness scripts, in contrast to those of intermediates, are integrated knowledge structures, accessible along multiple ways, an interaction between expertise level and instructional probe on the information produced will be expected: Subjects at both ends of the expertise scale will tell "the same kind of

11 To further avoid this cumbersome expression, these subjects will be called "interng." 
story," regardless of the way they are probed, while subjects at the inter. mediate levels, whose illness scripts are still rather sketchy and loosely structured, will be much more sensitive to the content of the instruction.

4) Regardless of "formal" measures of expertise, a positive relationship will be found between number of patients seen with a specific disease and the number of Enabling Conditions mentioned (and, conversely, a negative relationship between number of patients seen and amount of biomedical, Fault-related information).

5) Though the illness script theory does not make predictions about it, according to the results of Hobus et al. (1989), the amount of non-model information (e.g., statements about course, therapy, diagnosis, and frequency of occurrence of a disease) might be expected to increase with level of expertise.

\section{METHOD}

\section{Subjects}

Subjects were 23 fourth-year students, 22 sixth-year students, 23 interns in the second year of their internship and 22 family physicians. The fourth-year students had no or negligible experience in a practical clinical setting, while the sixth-year students, interns and physicians had on the average respectively 2 years, 5.5 years and 13.9 years experience in such settings. All subjects were either studying at the University of Limbarg at Maastricht (the Netherlands) or practicing in the south-eastern part of the Netherlands. Four physicians were working in a practice in Belgium, not far away from Maastricht.

\section{Material}

The stimulus material consisted of 20 names of diseases, the same as were used in the study of Hobus et al. (1989). Earlier experiments had shown that contextual information could play a facilitative role in the activation of accurate diagnostic hypotheses for these diseases (Hobus et al., 1987). Furthermore, the diseases selected displayed substantial variance as far as seriousness of the illness, afflicted organ system and frequency of occurrence in real-life situations are concerned. The names of the diseases are depicted in Table 3.1. Two diseases ( $A$ and $B$ in Table 3.1) were used for practicing purposes only. Disease 9 (secondary enuresis nocturna) was excluded from the analysis because there was considerable confusion among the subjects with respect to the nature of this affliction, i.e., some subjects expanded on "primary enuresis nocturna," while others searched for diseases enuresis nocturna might be a consequence of.

The materials also included a "patient frequency inventory." This was a sheet of paper on which the names of the 20 diseases selected for the present study were printed, each accompanied by a small rating scale. For each disease, subjects had to indicate on the rating scale how many patients they had seen with that disease, according to their own estimation. The rating scale was in fact a 10point scale, with values ranging from zero to $20^{+}$, the latter meaning: twenty or more patients seen. Figure 3.1 shows an example of the rating scale used in the inventory. Subjects were asked to take into regard only those cases seen in an actual clinical situation, and to discard all other instances (e.g., relatives or friends who might have the disease). Furthermore, if they were unable to decide on how many patients they had seen with a particular disease, it was allowed to 
mark two adjacent points on the scale. Finally, it was stressed that the purpose of the scale was to mersure separate, individual patients seen, so the same patient seen twice or more should only be counted as one instance of the disease.

Table 3.1

Names of the diseases used in the study.

A. Metastatic sigmoid cancer (metastases in the lungs)

B. Dyspepsia on a nervous basis (also called: nervous gastritis)

01. Aneurysm of the aortic artery (threatening rupture)

02. Urosepsis

03. Dermatitis peri-oralis

04. Vaginal Candidiosis

05. Perforated otitis media

06. Kidney stones colic

07. Carcinoma of the head of the pancreas

08. Stomatitis aftosa (multiple small ulcers in the mouth)

09. Secondary enuresis nocturna

10. Digitalis intoxication

11. Epidural hematoma

12. Nervous abdominal pain

13. Pediculosis pubis

14. Herpes zoster

15. Meningitis or encephalitis as a complication of mumps

16. Hepatitis A

17. Monilia of the mouth

18. Pre-infarct syndrome

\section{Procedure}

Subjects were, in order of participation, assigned to one of the two conditions. In the "prototype"-condition, they were asked to describe prototypical patients for each of the 20 diseases, in the "clinical picture"-condition they had to describe the clinical picture of each of the 20 diseases. A small pilot study had indicated that these instructions were generally well comprehended; if subjects asked informative questions (e.g., "Should I tell something about the treatment"), which occurred relatively often, the experimenter's standard reply was: "Yes, if you think that is part of the clinical picture / a prototypical patient" (depending on the experimental condition the subject was assigned to). That subjects do not have any trouble with describing a typical case for a certain disease is in line with Clancey's (1984) findings. Appendix 3.A shows some examples of subjects' descriptions. If a subject's description suggested that he had the wrong disease in mind, he was interrupted and corrected by the experimenter; to avoid misunderstandings, some of the diseases were announced by their "official" medical name as well as by a more vernacular description. Any information given about unintended diseases was discarded.

Subjects were tested individually. All narratives were audiotape recorded; there were no time constraints on the duration of either the entire session or the individual disease-descriptions. The modal duration of a session was 20-30 minutes. At the end of the session, the patient frequency inventory was presented to 
the sixth-year students, the interns, and the family physicians (the fourth-year students were expected to have no experience with any of the diseases).

\begin{tabular}{|l|l|l|l|l|l|l|l|l|l|}
\hline 0 & 1 & 2 & 3 & 4 & 5 & $6-10$ & $11-15$ & $16-20$ & $20^{+}$ \\
\hline
\end{tabular}

Figure 3.1. Example of a rating scale of the patient frequency inventory.

\section{Analysis}

Verbatim protocols were derived from the audiotape recordings. Protocol statements were classified into four categories: Enabling Conditions, Fault, Consequences, and "CDFT"-items. This latter category, which coincides with the non-model items in the Hobus et al. (1989) study, included four types of statements: about the expected course (e.g., "this patient usually ameliorates within a few days"), about diagnostic activities to be performed (e.g., "you can only diagnose this positively by taking a biopt"), about frequency of occurrence (e.g., "you don"t see that very often"), and about treatment (e.g., "it is difficult to treat"); hence, the indication CDFT-category. As the number of items in this category was too small to split up, it will be dealt with as one, be it miscellaneous, category.

An expert physician, who had experience with the illness-script categories, was consulted in order to establish a sound basis for the classification. Generally, not many difficulties were encountered during categorization. If in serious doubt, statements were omitted from the analysis; but this was hardly ever necessary. Sometimes, categorization of a specific item was determined by the context in which it appeared: for example, "hypercholesterolemia" was classified as Enabling Condition when it occurred in a statement like: "...these are patients known with sustained elevated cholesterol levels," but as a Fault item when it appeared in a statement like: "...this is caused by the formation of plaque at the walls of the coronary arteries, as a result of elevated cholesterol levels." It should be emphasized that statements were classified into one of the illness script categories, regardless whether they were correct or incorrect, from a medical point of view, with respect to the they were mentioned for.

As free production tasks usually result in large inter-individual differences in total number of statements, analyses were performed on the actual number of statements in each category, as well as on the respective proportions. Since proportions always add to one and thus are not independent, the results of this latter analysis should be interpreted with caution. Number and proportion of statements in each of the illness-script categories were analyzed by means of a 4 (levels of expertise) by 2 (probes) analysis of variance.

As far as the data of the patient frequency inventory concerns, the number of patients seen by each subject for each of the diseases was determined. If subjects had marked the categories 6-10,11-15, or 16-20, the arithmetic mean of the respective category (i.e., 8,13 , or 18 ) was taken as the number of patients seen by that subject. Subjects who had marked the category $20^{+}$always were assigned the value 20. If subjects had marked two adjacent categories, the arithmetic mean of the two categories was assigned. An estimation of the total number of patients seen was computed for each subject by adding the values over the 17 experimental diseases included. Pearson correlations were computed between the number of patients seen and the number of statements in each of the illness script cate- 
gories; as there was definitely a restriction of range effect, particularly for infrequent diseases and for subjects at the lower levels of expertise, the total number of patients seen by the subjects, rather than the values for individual diseases, was used in the analysis. Finally, a correlational analysis of the patient frequency data based on diseases, rather than subjects, was performed: Over 17 diseases, the average number of patients seen by subjects at each expertise level was correlated with the number of statements in each of the illness script categories.

\section{RESULTS}

\section{Number of statements in different illness script categories}

For the 17 experimental diseases included in the analysis, subjects mentioned on the average 9.97 statements per disease. Table 3.2 . shows the results, as far as total number of statements produced is concerned, for the four expertise levels and the two probe types. Analysis of variance revealed a borderline significant effect of expertise level on the total number of statements produced, $F(3,82)=$ $2.452, p<.07, M S_{\mathrm{e}}=9.777$, but no significant effect of experimental condition, nor a significant interaction between expertise level and experimental condition. From Table 3.2 it can be read that fourth-year students and interns were somewhat less prolific than sixth-year students and family physicians. However, the data do not show an interpretable linear or monotonic trend, nor is there any evidence for the existence of a so-called intermediate effect. Thus, it may be concluded that no easily interpretable relationship between level of expertise and verbal output was found, and that the two different types of cues used in the study did not lead to differences in the amount of information provided by the subjects.

\section{Table 3.2}

Average total number of statements mentioned for each expertise level and experimental condition.

Expertise level

Condition 4th-y. std. 6th-y. std. Interns Fam. phys. Means

\begin{tabular}{rccccc} 
Prototypical patient & 8.34 & 11.06 & 9.78 & 11.13 & 10.06 \\
Clinical picture & 9.12 & 10.85 & 9.33 & 10.25 & 9.88 \\
\hline Means & 8.72 & 10.96 & 9.57 & 10.73 & 9.97 \\
\hline
\end{tabular}

\section{NUMBER OF ENABLING CONDITIONS MENTIONED}

Analysis of variance with the number of statements categorized as Enabling Conditions as dependent variable showed a significant main effect of expertise level, $F(3,82)=8.831, p<.0001, M S_{\mathrm{e}}=.877$, a significant main effect of condition, $F(1,82)=24.303, p<.0001, M S_{e}=.877$, and a significant interaction between expertise level and condition, $F(3,82)=2.548, p<.05, M S_{\mathrm{e}}=.877$. Table 3.3 shows that 
the number of Enabling Conditions mentioned increases with experience, at least up to the level of the interns. Furthermore, the instruction to describe a prototypical patient led to twice as many Enabling Conditions mentioned than the instruction to describe the clinical picture: Under the latter instruction, about one Enabling Condition was mentioned for every disease, while under the former instruction, on the average more than two Enabling Conditions were produced. In addition, the finding of a significant interaction suggests that the effect of the experimental instruction differed between expertise levels. Table 3.3 shows that while the number of Enabling Conditions mentioned in the "prototypical patient" condition seems to level off at the more advanced levels of expertise, the number of Enabling Conditions mentioned increases monotonically with level of experience when subjects are instructed to describe the clinical picture associated with a disease. These data tend to support our hypothesis that preclinical students show a certain lack of knowledge of Enabling Conditions, or at least that they are less inclined to mention them even if probed to do so in a free-production task, while subjects at the intermediate levels do possess knowledge of relevant contextual factors, but tend to volunteer this knowledge mainly when more or less explicitly incited to. Experienced family physicians, on the other hand, end up with a relatively large number of Enabling Conditions even if cued toward different aspects of their illness scripts.

Table 3.3

Average number of Enabling Conditions mentioned as a function of expertise level and experimental condition.

Type of probe

Expertise level Prototypical patient Clinical picture Means

Fourth-year students $(\mathrm{N}=23)$ .68 .80

Sixth-year students $(\mathrm{N}=22)$

.98

Interns $(\mathrm{N}=23)$

2.97

1.13

2.09

Family physicians $(\mathrm{N}=22)$

2.47

1.57

2.06

Means

2.17

1.08

1.63

\section{NUMBER OF FAULT ITEMS MENTIONED}

Analysis of variance with number of biomedical, Fault-related items as dependent variable showed a significant main effect of expertise level on the number of statements in this category, $F(3,82)=7.932, p<.0001, M S_{\mathrm{e}}=1.022$. However, neither a significant main effect of probe, nor a significant interaction between condition and expertise level was found. Table 3.4 shows, for both experimental conditions, a monotonic decrease with expertise level in the number of Faultstatements mentioned. These results are in line with the expectations: First, biomedical knowledge is particularly important at the lower levels of experience, and second, no significant differences in the number of Fault-items mentioned 
were found between the two probes, as neither of the two types of instruction cued subjects specifically to produce biomedical knowledge.

Table 3.4

Average number of Fault itens mentioned as a function of expertise level and experimental condition

Type of probe

Expertise level Prototypical patient Clinical picture Means

$\begin{array}{llll}\text { Fourth-year students }(\mathrm{N}=23) & 1.70 & 1.93 & 1.81\end{array}$

$\begin{array}{llll}\text { Sixth-year students }(\mathrm{N}=22) & 1.30 & 1.67 & 1.48\end{array}$

$\begin{array}{llll}\text { Interns }(\mathrm{N}=23) & .52 & .90 & .70\end{array}$

Family physicians $(\mathrm{N}=22)$

.52

.62 .57

Means

1.01

1.30

1.14

\section{NUMBER OF CONSEQUENCES MENTIONED}

Table 3.5

Average number of Consequences mentioned as a function of expertise level and experimental condition

Type of probe

Expertise level Prototypical patient Clinical picture Means

\begin{tabular}{rlll} 
Fourth-year students $(\mathrm{N}=23)$ & 5.47 & 5.74 & 5.60 \\
Sixth-year students $(\mathrm{N}=22)$ & 6.52 & 7.51 & 7.02 \\
Interns $(\mathrm{N}=23)$ & 5.38 & 6.54 & 5.94 \\
Family physicians $(\mathrm{N}=22)$ & 7.08 & 6.48 & 6.81 \\
\hline Means & 6.10 & 6.57 & 6.33 \\
\hline
\end{tabular}

Analysis of variance with number of Consequences in the protocols as dependent measure also showed a significant main effect of expertise level, $F(3,82)=$ $3.253, p<.05, M S_{\mathrm{e}}=3.081$, but neither a significant effect of condition, nor a significant interaction were found. From Table 3.5 it can be read that the number of Consequences provided by the subjects displays a pattern quite similar to the total number of statements produced: sixth-year students and family physicians provide about seven Consequences statements per disease, while fourth-year students and interns mention about six of this type of items. Contrary to the expecta- 
tions, the probe to describe the clinical picture of a disease did not result in a significantly larger number of Consequences produced; both instructions release on the average slightly more than six Consequences.

\section{NUMBER OF CDFT-ITEMS MENTIONED}

Table 3.6 shows that subjects generally mentioned few items in the CDFTcategory; for example, of the 23 fourth-year students included in the study, 11 did not mention any items at all in this category, for none of the diseases. Thus, the conditions for performing an ANOVA were not met, and we had to revert to nonparametric techniques. To investigate the effect of expertise level on the number of CDFT-items, a Kruskal-Wallis one-way analysis of variance by ranks was performed (cf. Siegel, 1956). This analysis showed a significant effect of expertise level ( $H$ corrected for ties $=9.715, d f=3, p<.05$ ). Thus, the finding of Hobus et al. (1989) that subjects at higher levels of expertise provide more "nonmodel"-information was corroborated by the present results, though, as Table 3.6 suggests, curiously enough the effect in our study should mainly attributed to the results of the fourth-year students in the prototypical patient condition and the family physicians in the clinical picture condition, i.e., by combinations of expertise level and instruction that were not included in the Hobus et al. (1989) experiment.

Table 3.6

Average number of CDFT-items mentioned as a function of expertise level and experimental condition.

Type of probe

Expertise level Prototypical patient Clinical picture Means

Fourth-year students ( $\mathrm{N}=23)$

.26

.77 .50

Sixth-year students $(\mathrm{N}=22)$

1.07

.70

Interns $(\mathrm{N}=23)$

.91

.75

.84

Family physicians $(\mathrm{N}=22)$

1.06

1.58

1.30

Means

.82

.94

.88

To investigate the effect of experimental condition, a Mann-Whitney U-test for two independent groups was performed; this test revealed no significant differences between the two probes. Thus, the instruction to describe a prototypical patient did not result in a significantly different number of statements in the CDFT-category, compared to an instruction to describe the clinical picture. 


\section{Proportion of statements in different illness script categories}

\section{ENABLING CONDITIONS AS PERCENTAGE OF TOTAL NUMBER OF STATEMENTS MENTIONED}

Analysis of variance showed a significant main effect of expertise level, $F(3,82)=9.88, p<.0001, M S_{\mathrm{e}}=69.228$, a significant main effect of condition, $F(1,82)$ $=37.025, p<.0001, M S_{\mathrm{e}}=69.228$, and a significant interaction between expertise level and condition, $F(3,82)=3.815, p<.05, M S_{e}=69.228$ on the proportion of statements in the Enabling Conditions category. Table 3.7 shows that the percentage of Enabling Conditions generated by the subjects increases with experience till the level of the interns, but levels off for the experienced family physicians. Table 3.7 also shows that the finding of a significant interaction in this case means that in the prototypical patient condition, an inverted-U type of relationship between expertige level and percentage Enabling Conditions seems to hold, whereas in the clinical picture condition the percentage Enabling Conditions seems to increase monotonically with experience. A separate T-test for the interns and family physicians in the proto-type condition showed that the decline in proportion Enabling Conditions at the highest level of experience indeed was significant $t(22)=$ $2.578, p$ (one-tailed $<.01$. Therefore, it can be concluded that, if instructed to describe a prototypical patient with a disease, experienced physicians mention proportionally less Enabling Conditions than beginning family physicians, while the data support the notion the reverse is the case for the instruction to describe the clinical picture of a disease.

Table 3.7

Average percentage of items in the Enabling Conditions category as a function of expertise level and experimental condition.

Type of probe

Expertise level Prototypical patient Clinical picture Means

\begin{tabular}{rrrr} 
Fourth-year students $(\mathrm{N}=23)$ & 11.8 & 6.7 & 9.3 \\
Sixth-year students $(\mathrm{N}=22)$ & 20.5 & 8.6 & 14.6 \\
Interns $(\mathrm{N}=23)$ & 31.3 & 11.6 & 21.9 \\
Family physicians $(\mathrm{N}=22)$ & 22.2 & 15.5 & 19.2 \\
\hline Means & 21.7 & 10.5 & 16.3 \\
\hline
\end{tabular}

\section{FAULT ITEMS AS PERCENTAGE OF TOTAL NUMBER OF STATEMENTS MENTTONED}

Analysis of variance showed a significant main effect of expertise level, $F(3,82)=17.316, p<.0001, M S_{\mathrm{e}}=53.772$ on the percentage of Fault-items mentioned, but neither a significant effect of condition, nor a significant interaction between condition and expertise level. Table 3.8 shows the results. Analysis of the 
proportion of items in the Fault category yielded essentially the same results as the analysis of the absolute number of statements in this category: In both cases, a decrease of the contribution of Fault items with increasing levels of expertise was found, at least up till the level of interns, for both experimental conditions. These results are completely in line with the predictions.

Table 3.8

Average percentage of items in the Fault category as a function of expertise level and experimental condition.

Type of probe

Expertise level Prototypical patient Clinical picture Means

\begin{tabular}{rrrr} 
Fourth-year students $(\mathrm{N}=23)$ & 18.5 & 19.6 & 19.1 \\
Sixth-year students $(\mathrm{N}=22)$ & 10.5 & 13.7 & 12.1 \\
Interns $(\mathrm{N}=23)$ & 4.6 & 9.4 & 6.9 \\
Family physicians $(\mathrm{N}=22)$ & 4.3 & 5.7 & 4.9 \\
\hline Means & 9.3 & 12.3 & 10.7 \\
\hline
\end{tabular}

CONSEQUENCES AS PERCENTAGE OF TOTAL NUMBER OF STATEMENTS MENTIONED

Table 3.9

Average percentage of items in the Consequences category as a function of expertise level and experimental condition.

Type of probe

Expertise level Prototypical patient Clinical picture Means

$\begin{array}{llll}\text { Fourth-year students }(\mathrm{N}=23) & 66.3 & 66.6 & 66.4\end{array}$

$\begin{array}{llll}\text { Sixth-year students }(\mathrm{N}=22) & 60.6 & 72.0 & 66.3\end{array}$

$\begin{array}{llll}\text { Interns }(\mathrm{N}=23) & 55.7 & 71.9 & 63.5\end{array}$

Family physicians $(\mathrm{N}=22)$

65.4

62.0

63.8

Means

61.9

68.3

65.0

Analysis of variance revealed no significant main effect of expertise level on the percentage of Consequences in the protocols, but a significant effect of experimental condition, $F(1,82)=4.768, p<.05, M S_{\mathrm{e}}=173.091$, and a significant interaction between expertise level and experimental condition, $F(3,82)=2.794, p<.05$, 
$M S_{\mathrm{e}}=173.091$, was found. From Table 3.9 it can be read that subjects presented with the clinical picture probe mentioned on the average proportionally more Consequences, but also that this effect can be completely accounted for by the subjects at the intermediate levels of expertise. Thus, as far as the proportion of Consequences is concerned, subjects at the inter-mediate levels of expertise are more sensitive to experimental instruction than subjects at the more extreme levels. These results are not in line with those of the absolute number of Consequences, for which a main effect of expertise level, but no significant effect of type of probe, nor a significant interaction was found.

\section{CDFT-ITEMS AS PERCENTAGE OF TOTAL NUMBER OF STATEMENTS MENTIONED}

As the percentage of items in the CDFT-category did not even remotely resemble a normal distribution, the percentages were transformed into their square root values before being further analyzed. Subsequent ANOVA showed a significant main effect of expertise level, $F(3,82)=3.94, p<.05, M S_{\mathrm{e}}=1.491$, but no significant effect of experimental condition, and a merely borderline significant interaction between expertise level and experimental condition, $F(3,82)=2.21, p<$ $.10, M S_{\mathrm{e}}=1.491$. Table 3.10 shows that the experienced family physicians on the average produced relatively many CDFT-items, but the data also strongly suggest that this effect was for the better part due to the results of these subjects in the clinical picture condition. Like the results of the analysis of the absollute number of CDFT-items, the present analysis also support the hypothesis that experienced physicians mention more nonmodel items (cf. Hobus et al., 1989) than less experienced subjects.

Table 3.10

Average percentage of items in the CDFT category as a function of expertise level and experimental condition.

Type of probe

Expertise level Prototypical patient Clinical picture Means

Fourth-year students $(\mathrm{N}=23)$

3.4

7.1

5.3

Sixtli-year students ( $N=22)$

8.4

5.7

7.1

Interns $(\mathrm{N}=23)$

8.4

7.1

7.8

Family physicians $(\mathrm{N}=22)$

8.1

16.8

12.0

Means

7.1

9.0

8.0

In summary, the data on the number and percentage of statements in the different illness script categories as a function of expertise level show quite a mixed picture: some predictions were clearly borne out, while others were not; in addition, some rather unexpected results were found. The decrease, with expertise level, of both the absolute number and proportion of Fault-related items, and the concomitant increase in number and proportion of items on course, diag- 
nosis, frequency, and therapy, was completely in line with the expectations. As far as the number of Consequences is concerned, the predicted effects of level of experience (i.e., the intermediate or inverted-U effect) and type of probe (i.e., more Consequences when asked to describe the clinical picture) did not materialize, though an analysis of the proportion of Consequences suggested the opposite, i.e., expected, results. The results of the analysis of the number and proportion of Enabling Conditions were rather surprising: if asked to describe a prototypical patient with a disease, subjects at intermediate levels of expertise were surely not inferior to the experienced physicians; in fact, the interns may even have outperformed the experts in this condition. However, if asked to describe the clinical picture associated with a disease, a monotonic increase in the number and proportion of Enabling Conditions mentioned with expertise level was found. These latter results also imply that especially as far as Enabling Conditions are concerned, subjects at intermediate levels of expertise are more sensitive to type of instruction than subjects at the two extreme levels of expertise.

\section{Relationship between actual clinical experience with the diseases and quality of the descriptions}

For each individual disease as well as for the three relevant expertise levels, results of the patient frequency inventory are depicted in Appendix 3.B. Analysis of the data revealed that sixth-year students, interns, and experienced physicians had, on the average, encountered 65,155 , and 257 patients with the diseases included in the study (including the two practice diseases, but omitting the secondary enuresis nocturna disease). As subjects were not required to differentiate between frequencies of patient encounters above 20 , particularly the number of patients seen by the experienced physicians may be a gross underestimation. Not surprisingly, there are large differences between the frequency with which the individual diseases are seen in daily clinical life. Afflictions like dyspepsia on a nervous basis, perforated otitis media, and vaginal candidiosis have high frequencies of occurrence, while a threatening rupture of an aneurysm of the aortic artery, carcinoma of the head of the pancreas, and epidural hematoma are rare, and even experienced physicians may never have seen a patient with one of these diseases. Appendix 3.B also shows the number of experienced physicians that indicated, for each of the diseases, "never encountered" and "seen 20 or more times," respectively. Moreover, it also appears that diseases frequently seen by experienced physicians, are in general also relatively frequently seen by subjects at the lower levels of expertise; in other words, subjects at all three levels of expertise are exposed to approximately the same population of patients, as far as the diseases included in the present study concerns.

It would not be too far-fetched to assume that subjects are able to produce more information about diseases that they have seen often than about diseases they only know from study books or "hearsay." In addition, the illness script theory would predict that particularly the number of Enabling Conditions mentioned increases when subjects are more familiar with a particular disease, because practical clinical experience is required to integrate this type of knowledge into illness script structures.

As already indicated in the analysis section, these hypotheses were tested from two different points of view. In the first approach, the relationship between subjects' clinical experience in general (as estimated by the total number of patients seen with the experimental diseases) and number of statements in the 
different illness script categories was computed. Pearson correlation coefficients were computed between this measure of clinical experience and the number of statements in each illness script category, separately for each expertise level as well as for all subjects, except for the fourth-year students, who did not fill in the patient frequency inventory because they had virtually no clinical experience. The results are depicted in Table 3.11.

Table 3.11

Pearson correlation coefficients between clinical experience (as measured by tatal number of patients seen with the 17 experimental diseases) and number of statements in respective illness script categories, for three different expertise levels. The correlation coefficients are computed with subjects as basis.

Number of statements in illness script categories

\begin{tabular}{rccccc} 
Level of expertise & En.Con. & Fault & Conseqs & CDFT & N total \\
\hline Sixth-year students $(\mathrm{N}=22)$ & $.63^{*}$ & -.11 & .09 & .14 & .26 \\
Interns $(\mathrm{N}=23)$ & .10 & -.07 & -.28 & -.02 & -.13 \\
Family physicians $(\mathrm{N}=22)$ & .01 & .02 & -.08 & .05 & -.03 \\
\hline All subjects $(\mathrm{N}=67)$ & .24 & $-.37^{* *}$ & -.07 & .18 & -.01 \\
\hline
\end{tabular}

a total = total number of statements mentioned

* significant at the .01 level (one-tailed)

** significant at the .005 level (one-tailed)

Table 3.11 shows that only two correlations are significant, i.e., the correlation between clinical experience and number of Enabling Conditions mentioned by sixth-year students, and the correlation between clinical experience and the number of Fault-items mentioned over all subjects. Apparently, for sixth-year students, a positive relationship exists between the amount of clinical experience in general and the number of Enabling Conditions in the protocols, while for the interns and family physicians, no such relationship can be found. It was also analyzed whether the correlation found could be attributed to one of the two experimental conditions, or would hold for both. Though the correlation was .70 for sixth-year students in the prototypical patient condition, and .35 in the clinical picture condition, the difference between these two values was only borderline significant, $Z=1.54, p<.07$. Hence, we can not reliably attribute the relationship between clinical experience and number of Enabling Conditions in the protocols of the sixth-year students to a specific experimental condition. Nevertheless, the data on the Enabling Conditions depicted in Table 3.11 support the hypothesis that knowledge of Enabling Conditions is associated with practical clinical experience. The data also show a certain negative relationship between clinical experience and number of Fault items mentioned, a finding that is also in line with the hypothesis that the importance of biomedical knowledge, at least in clinical situations, decreases with increasing experience. 
In the second approach, it was investigated whether for the sample of diseases used in the present study, relationships would exist between the average number of patients seen with the diseases by subjects at a particular level of experience, and the average number of items mentioned by them in the different illness script categories. Thus, again correlation coefficients were computed, but this time based on diseases, rather than subjects. This procedure was performed as a substitute for subject-based correlations between number of patients seen and number of statements in different illness script categories for each individual disease; restriction of range effects would prevent the actual relationships being revealed. If positive correlations would be found in the present sample of diseases between the mean frequency of patient encounters and, for example, the mean number of Enabling Conditions mentioned by subjects at a particular level of experience, then this might be interpreted also as supporting the hypothesis that the integration of Enabling Conditions in knowledge structures should be considered a consequence of actual experience with diseases.

Table 3.12

Pearson correlation coefficients between the average number of patients seen with each of diseases by subjects at the particular expertise level, and the average number of statements mentioned for each of the diseases in the respective illness script categories by subjects at that specific expertise level. Computed over $N=17$ experimental diseases.

Average number of statements in illness script categories

Level of expertise En.Con. Fault Conseqs CDFT $N$ total ${ }^{\mathrm{a}}$

\begin{tabular}{rccccc} 
Sixth-year students $(\mathrm{N}=22)$ & $.44^{\circ}$ & .12 & .29 & .22 & .40 \\
Interns $(\mathrm{N}=23)$ & $.50^{*}$ & -.05 & .13 & $.43^{\circ}$ & .33 \\
Family physicians $(\mathrm{N}=22)$ & .35 & -.24 & .21 & .29 & .34 \\
\hline All subjects $(\mathrm{N}=67)$ & $.44^{\circ}$ & -.15 & .24 & .29 & .34
\end{tabular}

a $\mathrm{N}$ total $=$ total number of statements mentioned

" significant at the 11 level (one-tailed)

* significant at the .05 level (one-tailed)

The results are presented in Table 3.12. The higher a correlation value in the cell entries of this table, the stronger the general association between number of patients seen with a disease (by subjects at that specific expertise level) , and the number of statements mentioned in the particular illness script category, for that disease. Table 3.12 suggests that at all three expertise levels included, a certain positive relationship exists between the number of patients subjects have seen with a particular disease and the number of items mentioned in the Enabling Conditions category, while such a tendency can not be discerned for items in the other categories, though the possibility of a similar relationship between number 
of patients seen and items mentioned in the CDFT-categary can not be excluded. Like the previous analysis, these results support the hypothesis that actual experience with diseases is positively related to inclusion of Enabling Conditions information in the illness script structure: Generally, if subjects have seen more patients with a discase, they will also tend to mention more Enabling Conditions for that disease.

\section{EFFECTS OF YEARS OF MEDICAL EXPERIENCE ON NUMBER OF STATEMFNTS IN DTFERENT OLNESS SCRIPT CATEGORIES}

An altemative to the hypothesis that illness scripts develop as a consequence of practical clinical experience might be that this development is primarily a consequence of general experience, i.e., clinical as well as non-clinical, in the field. Though this supposition was already tested in the sections on the relationship between expertise level and number and proportions of statements in the different illness script categories, a more fine-grained analysis is possible if we take the actual experience of subjects, expressed as the amount of time spent in the medical domain, into consideration. Thus, correlation coefficients were computed between experience, expressed as number of months working in the medical domain, and the number of statements mentioned in the different illness script categories to the atatements in the protocols. Since this experience variable only differentiated between interns and experienced physicians - the sixth-year students all had approximately 18-20 months experience, and the fourth-year students no experience at all - only the two most advanced levels of expertise were included in this analysis.

\section{Table 3.1 .3}

Pearson correlation coefficients between amount of experience (expressed in months) and number of statements in the respective illness script categories, for two expertise levels.

Average number of statements in illness script categories

\begin{tabular}{rccccc}
\cline { 2 - 5 } Level of expertise & En.Con. & Fault & Conseqs & CDFT & $\mathrm{N} \mathrm{total}^{\text {a }}$ \\
\hline Interns $(\mathrm{N}=23)$ & -.32 & -.15 & -.26 & -.35 & $-.40^{\circ}$ \\
Family physicians $(\mathrm{N}=22)$ & -.17 & -.17 & $-.38^{\circ}$ & -.03 & -.34 \\
\hline All subjects $(\mathrm{N}=45)$ & -.14 & -.19 & -.11 & .08 & -.13
\end{tabular}

\footnotetext{
${ }^{a} \mathrm{~N}$ total $=$ total number of statements mentioned

${ }^{\circ}$ significant at the 1 level (one-tailed)
}

Table 3.13 shows the results. Though none of the correlation coefficients reaches significance at the .05 level, and only two at the .10 level, there is a general flavor of a weak negative relationship between years of experience and num- 
ber of statements in any of the illness script categories. This finding is consistent with earlier studies which report a decrease in amount of information mentioned by expert physicians, compared to intermediates (cf. Schmidt \& Boshuizen, $1993 b)$.

The fact that the number of Enabling Conditions shows, if anything, a negative association with medical experience as measured by time working in the field is quite telling: A positive correlation would have been expected, especially for the total group. Therefore, Hobus et al.'s (1989) conclusion that years of experience in the field is an important determinant in the development of full-fledged illness scripts does not seem to be warranted: Rather, it is personal experience with real. life patients that matters.

\section{DISCUSSION}

The inclusion of two additional expertise levels and an additional experimental condition into a replication of the Hobus et al. (1989) study yielded a considerably more complicated picture than the original study. By including four expertise levels, we were able to investigate the developmental trend of illness scripts at a more fine-grained level, and to discover whether the development of the different illness scripts components showed monotonically increasing, monotonically decreasing, (inverted-)-U-shaped, other, or no relationship at all with expertise level. The inclusion of two types of probes made it possible to find out whether subjects are sensitive to these differences in instruction. In addition, the simultaneous inclusion of expertise level and type of probe as experimental variables enabled us to detect interactions between these factors; by investigating the effects of this interaction for four different illness script components, an even more detailed picture could be given. Finally, by asking subjects how much practical experience they had with the different diseases included in the study, it was possible to look whether illness script development was determined by experience with specific diseases, rather than by expertise level in general.

The most salient difference between the present results and those of Hobus et al. (1989) is the failure of the present study to replicate the increase, with expertise level, of the number and proportion of Enabling Conditions mentioned when asked to describe a prototypical patient with a particular disease. The inclusion of interns in our study suggests that the number and proportion of Enabling Conditions mentioned in a free production task either remains approximately constant for subjects from about 18 months experience onwards, as the data on the absolute number of Enabling Conditions suggest, or shows an inverted-U effect, with a maximum for subjects having about 5.5 years clinical experience, as the data on the proportion of Enabling Conditions mentioned indicate. However, if asked to describe the clinical picture associated with a particular disease, indeed a significant increase in number of Enabling Conditions mentioned with expertise level was found. As these results were found for both the absolute number and the proportion of Enabling Cionditions in the descriptions, they are not an artefact of verbal fluency.

Also in contrast with the Hobus et al. (1989) study, we found a decrease in the number and proportion of Fault-statements mentioned with increasing expertise level; however, this finding is in line with the illness script theory, which predicts a decreasing emphasis of biomedical knowledge when subjects become more experienced. The absence of a significant interaction between expertise level and experimental condition for Fault-items indicates that this decrease holds for both 
types of probes; this is also something that was expected, because neither the instruction to describe a prototypical patient, nor the instruction to describe the clinical picture tunes the subjects specifically towards mentioning biomedical knowledge.

Furthermore, the average number of Consequences mentioned in the present study was considerably larger than in the Hobus et al. (1989) study: In the former one, sixth-year students and experienced physicians mentioned 6.52 and 7.08 Consequences (ef. Table 3.5 ), respectively, while the corresponding values in the latter study amounted to 4.44 and 2.45 Consequences, respectively. As in the Hobus et al. (1989) study, in contrast with the present one, subjects were provided with the main complaint of the patient to be described, the finding that subjects in our study generally volunteered more Consequences did not come as a surprise. However, and more importantly, we did not find the expected decrease in number of Consequences mentioned by subjects at the highest level of expertise. In fact, the effects of both expertise level and type of instruction on number and proportion of Consequences in the protocols are difficult to interpret. We think it is hard to conceive of a theory that can explain the peculiar, indented (or $\mathrm{N}$-shaped) relationship between level of expertise and number of Consequences mentioned. Strikingly, the shape of this relationship parallels the shape of the relationship between expertise level and total number of items mentioned (cf. Tables 3.2 and 3.5). Thus, quantitative differences in werbah output may exert the largest influence on the Consequences category. It is also salient that the differences in proportion Consequences, over the two types of instruction, are extremely small; at: all levels of expertise, subjects" descriptions seem to consist for about two-thirds of Consequences (cf. Table 3.9).

Finally, the increase in the number of CDFT-(or nonmodel-) items found by Hobus et al. (1989) was also replicated in our experiment, though the effect in our study could not be accounted for by the two expertise levels that were similar to those in the former study; our data strongly suggest that, had we not included fourth-year students in our study, no significant effects of expertise level on number of CDFT-items would have been found. In general, the number and proportion of items in the CDFT-category were not dependent on the type of instruction, a finding that is not surprising, as neither of the instructions specifically tuned towards inclusion of CDFT-items in the descriptions. More fine-grained future research might disclose information about the relative importance of the four categories course, diagnosis, frequency, and therapy, in experts" and nonexperts' descriptions. For example, predictions about the future course of an illness may be used to test diagnostic hypotheses; it may be expected that experienced physicians are better able to make use of this "tool." Similarly, knowledge of the applicability of specific diagnostic procedures, of the frequency of occurrence of diseases, and of possible therapeutic interventions, may all be expected to increase with expertise level. Consequently, though the number of statements on each of these four CDFT-topics (course, diagnosis, frequency, and therapy) was small and hence prevents us from drawing definite conclusions about it, it does not seem unlikely that each of the four has had a certain contribution in the experts' higher scores on this category.

We think that in general, the present results are in support of the illness script theory. This theory states that in the early stages of medical expertise development, the basic sciences play an important role in constructing scripts for diseases. With increasing expertise, the role of this biomedical knowledge is deemphasized, while simultaneously the role of contextual factors of diseases becomes more important. This developmental trend is reflected by the "stories" 
tald by our subjects. While biomedical and pathophysiological information forms a substantial part of the stories told by fourth-year students, interns and family physicians provide hardly any information of this kind.

However, the results concerning the Enabling Conditions component forces us to modify previous conclusions. It should be kept in mind that Hobus et al. $(1987,1988,1990)$ found that experienced physicians' diagnostic performances increase considerably when contextual information about a patient was provided, compared to a situation in which this information was absent, while sixth-year students were unable to profit from this additional source of information. Thus, Hobus et al. (1988) concluded that the illness scripts of experienced physicians are enriched with an Enabling Conditions component, while sixth-year students still lack much of this knowledge. However, as we found that sixth-year students, compared to experts, do not lag behind in the number of Enabling Conditions produced if asked to describe a prototypical patient, it seems unlikely that lack of knowledge from the part of the former subjects is responsible for the Hobus et al. (1987, 1988, 1990) results. Together with these latter study, the present one suggests that knowledge accessibility, rather than knowledge availability, is the critical aspect. Sixth-year students know quite a lot about the importance of contextual information, but they are only able to access this knowledge when it is explicitly cued, for example, if they are instructed to describe a prototypical patient with a disease. In other circumstances, like in the diagnostic situation employed in the Hobus et al. (1988) study, or when asked to describe the clinical picture of a disease, like in the present study, Enabling Conditions are apparently out of reach for the non-experts. In the present study, this conclusion is evidenced by the interaction between expertise level and instruction: the "prototypical patient" instruction triggers Enabling Conditions, even at less advanced levels of expertise, while in the "clinical picture" condition, the number of Enabling Conditions volunteered increased monotonically with expertise level. In this condition, the importance of contextual information is less conspicuous, and as a consequence, this type of knowledge is less readily accessed by non-expert subjects. Thus, on basis of our results, and in light of the findings of Hobus et al. $(1988,1989)$, we consider it safe to conclude that though knowledge of contextual factors apparently is already present at lower levels of expertise - the fact that interns provided more than $30 \%$ Enabling Conditions in the "prototypical patient" condition, aggainst a good $20 \%$ for the experienced physicians - integration of this knowledge into illness script structures is a gradual process, extending well beyond graduation.

Though we have no direct evidence for it, it may even have been the case that, contrary to current opinions (e.g., Brooks, Norman, \& Allen, 1991), interns are even more apt to invoke the image of a specific, recently seen patient with a disease, than experienced physicians, who tend to rely more on "real" illness scripts, with irrelevant Enabling Conditions filtered out. If this turned out to be the case, then the non-experts would probably provide more Enabling Conditions that do not show a medically significant relationship with the disease in question. Though this explanation, i.e., that expert physicians' Enabling Conditions are qualitatively, rather than quantitatively, different from those of sixth-year students, can not be definitely excluded, the present data do not seem to support it: Age, sex, and medical history were by far the most frequently mentioned Enabling Conditions at all expertise levels, and there are no indications that there were qualitative differences in content of these Enabling Conditions between subjects at different levels of experience.

The present study also provides evidence that the integration of Enabling Conditions into illness scripts is a consequence of experience with real patients, 
rather than a general effect of expertise development (cf. Table 3.12). We consistently found moderately positive correlations between frequency of experience with diseases and number of Enabling Conditions mentioned. First, subjects' clinical experience in general, as measured by total experience with the diseases used in the present study - a measure that presumably relatively accurately reflects experience with patients in general - is positively correlated with the number of Enabling Conditions mentioned. Second, with diseases as basis, positive correlations were found between the number of patients seen by subjects at each of the expertise levels (though these correlations were significant only for the two intermediate levels), and the number of Enabling Conditions mentioned. In general, if a disease is quite often seen by subjects, relatively many Enabling Conditions will be mentioned when they are asked to describe a prototypical patient or the clinical picture of that disease, while no similar relationship was found between actual experience and number of items in the Fault-or Consequences category. These findings support the hypothesis that particularly Enabling Conditions gradually become integrated in illness script structures as a consequence of encountering actual patients. It is rather telling that no positive relationship was found between the more formal measure of medical expertise, i.e., amount of time working in the domain, and number of items in different illness script categories. As such, our results are in line with those of Weber et al. (1993), who found no general effect of expertise level on activation of diagnostic hypotheses, but instead a specific effect of availability of diagnosis, which may be considered a direct effect of experience with diseases.

A final remark should be made. In the present study, all diseases were considered to be equivalent; it was assumed that every subject had scripts for all of them, even if this would be often, especially for subjects at the lower levels of expertise, embryonic rather than full-fledged scripts. One might wonder what would happen if a subject had no script at all for a specific disease. Incidentally, the data on the discarded disease of "secondary enuresis nocturna" may provide some information about what might happen when no ready-made illness script structures are available. Though the number of Enabling Conditions mentioned for this affliction was by no means exceptional compared to other diseases $(1.90$ over all subjects, against an average of 1.63), the number of Fault-items mentioned was the highest of all diseases ( 2.63 over all subjects, against an average of 1.14), while the number of Consequences was the lowest of all diseases (2.61 over all subjects, against an average of 6.33 ). The number of CDFT-items mentioned for this disease was almost equal to the general mean (.87 over all subjects, against an average of .88). Apparently, subjects were trying to make sense of this disease, by using Fault-related, biomedical knowledge. The reason that the number of Enabling Conditions was still relatively high might be the particular nature of this affliction: the word "enuresis" typically invokes the image of a small child, and secondary enuresis (enuresis as a consequence of a physiological or anatomical abnormality) involkes the image of a female rather than a male, whereas primary enuresis nocturna is typically found in boys. 


\section{CHAPTER 4}

\section{THE INFLUENCE OF MEDICAL EXPERTISE AND CASE TYPICALITY ON CASE PROCESSING}

\section{INTRODUCTION}

In the previous chapter, it was concluded that the superior diagnostic performances of experts reported in the literature probably can be accounted for by the integration of Enabling Conditions into illness script structures. Intermediates first acquire knowledge of contextual factors and subsequently integrate it into their knowledge structures, to make it available for diagnostic purposes. Thus, there is a stage in which advanced students possess the relevant knowledge, but are not yet able to use it. The sixth-year students and interns are able to produce a large number of Enabling Conditions when specifically cued to provide this type of information: If the cue points into another direction, their performances show a significant drop. Apparently, their knowledge of Enabling Condi tions, though present in principle, is much less accessible in the absence of an appropriate cue. This phenomenon might also explain why subjects are unable to use contextual information in a diagnostic situation: As their knowledge of Enabling Conditions is not yet integrated in their developing illness scripts, they can not profit to the same extent as expert physicians from the availability of contextual information. In the previous chapter, it was also concluded that the whole process of integrating Enabling Conditions into illness script structures is, at least partly, a consequence of practical experiences with real patients.

In the present chapter, we want to elaborate further on the "script" aspect of the illness script. In the introductory chapter, an illness script was defined as an integrated knowledge structure, consisting of at least three, and probably four, components: the Enabling Conditions, the Fault, the Consequences, and a fourth, miscellaneous, component which contains mainly knowledge about disease course and therapy. As such, ilness scripts are complex knowledge structures; applicable in real-life situations. In cognitive psychology, a number of approaches have been developed to deal with complex knowledge in real-life situations; examples of such approaches are: situation models (Van Dijk \& Kintsch, 1983), frames (Minsky, 1975), schemas (Bartlett, 1932/1954), mental models (Gentner \& Stevens, 1983; Johnson-Laird, 1983), and scripts (Schank \& Abelson, 1977). An important difference between situation models and mental models on the one hand and schemas, scripts and frames on the other hand is that the former two emphasize the on-line construction of the relevant knowledge structures during problem solving, while the latter three tend to view these structures as relatively stable and precompiled, and hence are more inclined to stress application rather than construction.

As already discussed in Chapter 1 , research on categorization is also relevant for medical expertise, and particularly medical diagnosis. Diseases can be viewed as categories; patients as exemplars or instances that have to be categorized. Categorization theories usually deal mainly with categories that have a relatively simple structure, often based on perceptual aspects. Though diseases are complex entities, and diagnostic classification entails considerably more than 
categorization based on simple, straightforward, perceptual features, there are certain commonalities. For example, as diagnostic hypothesis generation is an important aspect of the early stage of a medical consultation (cf. Elstein et al., 1978), it is probably not too far-fetched to view it as a form of classification based on relatively few features, usually Enabling Conditions and presenting complaints. The subsequent process of diagnostic hypothesis verification, however, lends itself to a much lesser extent for description in terms of classification or categorization.

Most categorization theories emphasize the fact that generally, for reallife categories, not all instances or exemplars have the same status: some of them are "good", i.e., representative members, while others are not. For example, "car" and "plane" are good extmples of means of conveyance, while "camel" and "zeppelin" are not. Good examples of a category are also called prototypical examples (Smith \& Medin, 1981); their category membership is usually determined quickly in a decision task, and they receive high typicality ratings (Rosch, 1975). This prototypicality aspect of categories can easily be extended to the medical field, i.e., to the structure of the domain of diseases (Bordage, 1984; Bordage \& Zacks, 1984) as well as to individual patients as instances of a disease.

In the present chapter, an effort will be made to integrate two different views, script theory and categorization theories based on typicality, in order to account for the behavior of gubjects in medical diagnostic situations. As medical experts tend to employ, at least in routine diagnostic situations, pre-compiled, ready-made knowledge structures, rather than elaborate reasoning, approaches based on mental models or situation models are probably less appropriate to describe expert diagnostic behavior in these situations. Findings that the importance of biomedical reasoning decreases with increasing practical experience support this approach (e.g., Boshuizen \& Schmidt, 1992; Clancey, 1983; Patel \& Groen, 1986a; Patel et al., 1989).

Scripts (Schank \& Abelson, 1977) are defined as representations of standard event sequences; they enable people to make sense of their social environment by using previous experiences to predict what is likely to happen in a similar current situation. A salient feature of scripts is that this understanding and prediction occur almost without any effort, even in very complex situations. The core idea of scripts is that they provide connectivity between separate events or phenomena that can be interrelated by real-world, often social knowledge, as opposed to items that are connected by formal or syntactical rules, as is the case in mathematics or logic. In the Schank and Abelson (1977) approach, scripts are enacted by an actor, they consist of a sequence of events, (i.e., the time variable plays an important role), and they are part of a goal-directed structure. Scripts consist of slots that can be filled by incoming or default values; the actual range of values these slots may assume is prespecified. Scripts can be activated by hearing or mentioning the script title, script actions, or even specific individual slots, and instantiated by providing specific values to be filled in the slots. Scripts derive their practical power to a large extent from the possibility to fill slots by default. Or, in other words: An important feature of script instantiation is that, unless information is available that suggests the opposite, prototypical values are automatically inserted in the variable slots. As a consequence, instantiated scripts will often function as more or less prototypical exemplars of the more general category the script is about (cf. Abelson, 1981).

Illness scripts share a number of aspects with both the "classical" Schank and Abelson (1977) scripts and categories based on prototypes. Like categories, illness scripts are part of a taxonomic structure, and they are especially tuned to situations in which (quick) categorization is of importance. With classical scripts, 
they share the importance of a time variable, the presence of slots which can be filled by default, and the fact that there exists an underlying model or theory 12 .

However, some important differences between classical scripts and illness scripts, already alluded to in the introductory chapter, need to be further elaborated. First, script activation is non-problematic, or even obvious, for classical scripts, but not for illness scripts. In a classical script situation, activation of the script occurs immediately and effortessly, often after hearing or reading one statement (e.g., "John went to the dentist"). For illness scripts, activation of the appropriate script is the core problem; once an illness script, or, for that matter, a few illness scripts, are activated, the most crucial step in medical diagnosis is probably taken (cf. Cutler, 1979; Elstein et al., 1978; Wilkins, 1970 ).

Second, information that is inconsistent with a currently activated illness script will be dealt with differently, compared to information that violates a classical script. Information that contradicts an illness script will often lead to rejection of that script, or at least to a considerable drop in its likelihood. For example, if we suspect that a patient has Pfeiffer's disease, i.e., the illness script "Pfeiffer's disease" is currently activated, we will surprised to hear that he is 72 years old and shows shortness of breath; probably, this information will lower the activation of this illness script, and instigate a search for another, better fitting script. For classical scripts, on the other hand, inconsistent information will be tagged to the current instantiation of the script, rather than decrease its likelihood (cf. Graesser \& Nakamura, 1982). For example, the table cloth catching fire may be an event that is inconsistent with the expected continuation of the restaurant script, but it does not mean that the whole script is discarded in favor of another one. In summary, the point is that script competition is common in medical diagnosis, but not in daily social life: As a consequence, information that is at variance with the script will be handled differently in both cases. For illness scripts, it will decrease the probability that the particular script is actually applicable, while for classical scripts, it will be tagged as an unexpected, possibly interesting, event.

The issue of typicality, on the ather hand, is relevant for both classical scripts and illness scripts. Cases, as instantiations of illness scripts, can be more or less prototypical or atypical, depending on the typicality of Enabling Condutions and Consequences. Similarly, classical script instantiations can differ with

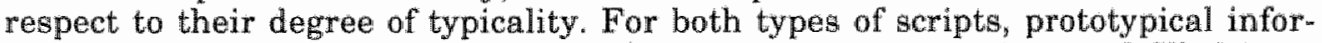
mation is information that is completely in line with expectations and filled in or inferred by default, if it is not explicitly mentioned. Atypical information deviates to a certain extent from expectations, but not to the point of refuting or obstructing a script; in this latter case, the information would be inconsistent, rather than merely atypical.

Both classical and illness scripts are activated as integrated wholes, in an all-or-none fashion. Thus, if a script is accessed, a large number of interconnected concepts becomes activated, though many of them probably only indirectly, as a consequence of default values being filled in slots. This indirect or implicit activation initially results in a prototypical instantiation of the seript. In other words: If a script is activated as a whole, its prototypical features and slot values are simultaneously also activated, even if no information about them is (yet) available (cf. Graesser et al., 1980; Maki, 1990; Nakamura \& Graesser, 1985; Yekovich \& Walker, 1986). Consequently, new incoming information that is in line with this

12 A number of recent categorization theories are also explicitly baged on the existence of an underlying theory or model (e.g., Medin \& Ortony, 1989) 
implicit prototypical instantiation of the script, will be processed with a minimum of effort: It is already activated. However, if atypical information is pregented, it has to be separately tagged to the script, or at least a corresponding default value has to be adjusted; this process will require some judgment, reflection, or elaboration, and, consequently, some extra time. An implication would be that processing script atypical information will take more reading time than processing script pratotypical information. Indeed, the hypothesis that reading times of text passages are influenced by typicality of the presented information has received experimental support. For example, Bellezza and Bower (1981) found that atypical script actions, in which objects or entities appeared that could not immediately be fit into script slots, took longer to process than typical script actions. In a similar vein, though with focus on categories rather than scripts, McKoon and Ratcliff (1989) found that statements in which a highly prototypical exemplar of a category appeared are processed faster than statements in which a more atypical exemplar was mentioned.

The main question addressed in the study presented in this chapter is: Do findings such as those of Bellezza and Bower (1981) also apply to the presumed illness scripts? Or, in other words, do subjects process information that is atypical for an activated illness script slower than information that is prototypical for it? And does this apply to the same extent for processing of Enabling Conditions and Consequences? In addition, it is also hypothesized that the flexibility of illness scripts is limited. While subjects will try to fit atypical information into the current script instantiation, at the expense of some additional processing time, blatantly contradictory information will result in quick rejection (de-activation) of the current illness script. If sufficiently inconsistent with this current script, contradictory information may even be processed relatively fast, i.e., lead to a quick rejection of the activated script, rather than to a time-consuming effort to incorporate or tag the information.

In addition, according to illness script theory, typicality of information will influence other variables as well. In medical practice, actual likelihood estimates are often assigned to illness scripts, if they perform the function of diagnostic hypotheses. We assume a monotonic relationship between the degree of typicality of the case to be diagnosed (i.e., the current instantiation) and the value of the illness script likelihood estimate. If this case is prototypical for the activated illness script, the likelihood that this script is appropriate will be high, whereas a more atypical patient description will lead to a more moderate degree of confidence in its applicability. Therefore, if asked to estimate the probability that a specific pacient suffers from a particular disease, physicians will assign a higher estimate if the patient" $\$$ context, signs and symptons are prototypical for that particular disease than if they are atypical for it.

Furthermore, the outcomes of the experiment described in Chapter 3 and related work in the field (e.g., Schmidt \& Boshuizen, 1993; Schmidt, Boshuizen, \& Hobus, 1988) suggest that the effect of typicality on processing speed and probability estimates might be mediated by expertise level. For example, the existence of an intermediate effect suggests that subjects at an intermediate level of expertise tend to rely on elaborate processing of medical information in order to comprehend and assimilate case information, while experienced physicians' comprehension is of a much more immediate nature (e.g., Schmidt \& Boshuizen, 1993b). Thus, it might be expected that inexperienced physicians generally take more time to process a case than expert physicians. Besides, evidence was found for the hypothesis that the integration of Enabling Conditions into illness script structures is a feature of particulary the more advanced stages of development of med- 
ical expertise. Hence, it may be expected that differences in case information processing times between experts and non-experts will be especially pronounced for the Enabling Conditions. Intermediates illness scripts will be relatively well developed with respect to Consequences; thus, prototypical Consequences information will be processed quickly by these subjects as well as by experts. Prototypical Enabling Conditions, on the other hand, will be processed quickly by experts, but more slowly by intermediates. Thus, typicality, expertise level, and illness script category will each exert an influence on case processing times, while an interaction between typicality of Enabling Conditions and expertise level might be expected: Experienced physicians will show larger differences in reading speed of prototypical and atypical Enabling Conditions than intermediates.

By the same token, it might also be expected that experienced physicians will be more inclined to consider both typicality of Enabling Conditions and typicality of Consequences in determining a probability estimate for a specific case, while advanced students will be more sensitive to typicality of Consequences, than to typicality of Enabling Conditions. Therefore, if a case description of a patient with prototypical Enabling Conditions and prototypical Consequences with respect to a particular disease is presented, experienced physicians will assign a higher probability to that disease than if either or both of these components are of a more atypical character. Students or inexperienced physicians, on the other hand, will be mainly sensitive to typicality of Consequences of a disease, as their illness scripts are relatively well developed with respect to this component. Prototypicality or atypicality of Enabling Conditions will not exert a large influence on their probability ratings.

An experiment was designed to investigate these predictions. Based on a number of different diseases, short case descriptions were constructed. Each case description consisted of a number of statements, in which information about Enabling Conditions or Consequences of the patient in question was provided. The case presentations were computer based; subjects had to read each case as quickly and thoroughly as possible. At the end of each case, they had to decide whether a particular illness script, activated at the beginning of the case by announcing the name of the disease, was applicable; finally, they had to estimate the probability, expressed as a percentage score, that the patient indeed suffered from this disease, or, in other words, that the illness script was pertinent to the case.

Subjects were provided with a press-button device to make their responses. Reading times for each individual statement, and probability estimates for the complete case, were recorded. The information in the cases was manipulated in such a way that the influence of prototypicality and atypicality of Enabling Conditions and Consequences on reading times and probability estimations could be experimentally investigated. By presenting case descriptions of different types to subjects at two different levels of expertise, i.c., sixth-year students and experienced family physicians, the following hypotheses were tested:

1) Experienced subjects will generally process case information faster than inexperienced subjects.

2) Completely prototypical cases, i.e., cases with prototypical Enabling Conditions as well as prototypical Consequences will generally be processed faster than completely atypical cases, i.e., cases with atypical Enabling Conditions as well as atypical Consequences; processing times of cases that are partly typical, partly atypical will fall somewhere in-between.

3) An interaction will be found between expertise level and typicality of Enabling Conditions: Experienced physicians will be sensitive to typicality of Enabling Conditions whereas non-experts will not, or only to a lesser extent, show such 
sensitivity. This will be evidenced by experienced physicians showing an additional advantage in processing speed for the prototypical Enabling Conditions parts of cases, compared to intermediates. Since for typicality of Consequences no such interaction will be expected, the results will show a three-way interaction between expertise level, typicality, and illness script component, an interaction that will be accounted for by the data on the Enabling Conditions.

4) Similar predictions will hold for the probability estimates: Completely prototypical cases will receive higher probability estimates than completely atypical cases, with the probability estimates of cases being partly prototypical and partly atypical will fall somewhere in between; for this measure, experienced physicians will also be more sensitive to prototypicality of Enabling Conditions than less experienced subjects, who will take mainly Consequences into consideration in determining how probable an instance a case is of a specific disease.

5) Cases in which information is provided that is contradictory to what would be expected given the disease the case is presumably an instance of, are processed relatively quickly, because in an early stage the decision is taken that the case is not an instantiation of the announced disease. Consequently, probability estimates for these cases will be close to zero.

\section{METHOD}

\section{Subjects}

Subjects were 30 sixth-year students from the Maastricht Faculty of Medicine and 30 experienced family physicians, recruited in one of three small towns in the south-eastern part of the Netherlands. The sixth-year students had either completed their residency or were about to complete it; they had on the average 2 years experience in a clinical setting ${ }^{13}$. The family physicians had on the average 11.75 years experience as general practitioners, ranging from 1 to 25 years, clerkship and postgraduate training years not included. As it takes at least two years additional education after medical graduation to become a family physician, the least experienced family physician has had at least 5 years experience in a practical setting, the clerkship years included.

\section{Material}

Based on 24 different diseases, case descriptions were constructed. With the exception of the disputable "secondary enuresis nocturna" affliction, the 20 diseases used in the Hobus et al. (1990) study, as well as in the previous chapter, were again exploited. Five additional illnesses were selected to complete the set of 24. Appendix 4.A shows the names of the diseases.

Each case description consisted of a number of statements. The statements provided information about the setting (e.g., consultation hour, emergency telephone call, request for a visit), the patient"s background, his or her introductory complaint, and some symptoms and findings. Every case description started with some information about the contextual characteristics of the patient in question, followed by the setting, one or more complaints and finally some symptoms and

13 The Dutch medical curriculum consists of a four-year training program in the biomedical and clinical sciences, followed by a txo-year clerkship program 
findings from the physical examination. The first three cases, which were used for training purposes only, were identical for all experimental conditions.

For 16 of the remaining 21 diseases, four different case variants were constructed. Each case variant consisted of a set of Enabling Conditions statements, followed by a set of Consequences statements. Such sets could be either prototypical or atypical. All four possible combinations were exploited: prototypical Enabling Conditions could be followed by either prototypical or atypical Consequences, and similarly, atypical Enabling Conditions could be followed by either prototypical or atypical Consequences. Case variants of these types were called $\mathrm{PP}, \mathrm{PA}, \mathrm{AP}$ and AA, respectively; thus, the first $\mathrm{A}$ or $\mathrm{P}$ of the type indication refers to the nature of the Enabling Conditions, the second to the nature of the Consequences. Prototypicaity and atypicality of case information was determined by analysis of the responses given by the subjects in the study described in the previous chapter: It was assumed that Enabling Conditions and Consequences that were mentioned by many of the experienced subjects describing a proto* typical patient with, or clinical picture of, a disease might be considered prototypical, while features mentioned by only few subjects would be atypical. The case variants constructed on basis of this material were checked by three experienced physicians, who were instructed to judge the case descriptions on basis of their typicality. Based on their judgements, some relatively minor modifications were made. It should be emphasized that the typicality manipulation in this study always refers to the complete set of Enabling Conditions or Consequences of a case, and not to individual case statements. It should also be stressed that typical and atypical sets remained constant over the case variants; e.g., the prototypical Enabling Conditions of a case were identical for both typicality variants of the following Consequences. Nevertheless, sometimes small adjustments were inevitable, for example, if the sex of the patient was different for prototypical and atypical Enabling Conditions.

An example of a disease with its four case variants can be found in Appendix 4.B. The total number of statements per case description ranged from 7 to 18 . The four different variants of each disease case description always contained the same number of statements, though the number of words could differ somewhat between these variants. In Appendix 4.C, some information about the length of the case descriptions is given.

As only one variant of each case could be presented to every subject, four different sequences of cases had to be constructed, with each of the four variants of any case (PP, AP, PA and AA) appearing in only one of the sequences. These sequences were constructed by assigning the 16 experimental diseases randomly to four groups of four diseases. For the first group, the four PP cases were assigned to one of these sequences, the AP cases to another one, and so on. This procedure was repeated for the other three groups of four diseases, under the constraint that in the end each experimental sequence consisted of four cases of each type. The order in which the individual cases appeared in the sequences was randomly determined, but identical for the four sequences and remained fixed during the experiment.

For the remaining five diseases, so-called "no-cases" (NO type cases) were constructed. These were cases in wich a patient was described with a complete ly different disease than the illness script header (i.e., the presumed diagnosis) suggested. This type of cases was added for two reasons: First, to contrast completely atypical (AA type) cases with obvious "no-cases", in order to invegtigate whether the AA type cases were having a certain plausibility with regard to the diseases they were based on, and second, to investigate whether processing of 
these cases would indeed proceed at higher speed, as the theory predicts, than processing of AA type cases. As there was only one variant of the NO type cases, they were the same for all. four sequences, and they were inserted at random positions in the sequences.

To control for possible order effects, an A-form and a B-form were constructed for each of the four case sequences. In the A-form of each sequence, first the cases 4-13 were shown, followed by the cases 14-24, while in the B-form this order was reversed. The practice cases 1-3 were always presented first. Appendix 4.D provides a complete overview of the design of the sequences.

Screen texts appeared in black on a white background (font: Palatino, size: 18); no colors were used. The text lines were centered at the middle of the screen. By using a press-button device with three buttons (a button labeled "yes", a button labeled "no", and an unlabeled button for proceeding through the case description), together with an experimental program called PET14, it was possible to measure reading times of individual statements with an accuracy of $1 \mathrm{msec}$. The main program was written in Authorware.

\section{Procedure}

According to the order of participation, subjects in both expertise groups were assigned to one of the eight case sequences. They were tested individually, the sixth-year students at the university office, and the family physicians in their consulting-room. Subjects were seated in front of a computer screen and given a short introduction. Next, the experimenter started the session by saying: "Read the first case and decide whether this is a patient with $X$ " ( $X$ being the disease on which the first case presentation was based). Subsequently, the experimenter started the case presentation and the first statement of the first case appeared on the screen. Subjects had to push a button on a press-button device to proceed through the case. They were instructed to read each case description as thoroughly and as fast as possible, and to judge whether the patient described in the case actually suffered from the disease announced by the experimenter at the beginning of the case presentation. Every time they pushed the button to proceed through the case description, the current case statement disappeared from the screen and the reading time for that particular statement was recorded. Subsequently, the next statement was presented.

At the end of every case description, subjects first had to answer a case eval. uation question, phrased as a forced-choice question: "Is this a patient with X, yes or no?" ("X" being the name of the disease announced at the beginning of the case presentation). Subjects could make their choice by pressing a "yes" or a "no" button on the press-button device. Next, they had to estimate the probability, expressed as a percentage value, that the presented case description actually matched a patient with the specified disease.

Reading times for all individual case statements were recorded. As these times were in the order of magnitude of at least 500-600 msecs, but often even lasted several seconds, between subject differences in the time needed to perform the physical response of pressing a button might be considered negligible. Reading times were also measured for the case evaluation question, but not for the probability estimations: Deciding on the "exact" quantitative size of a likelihood was not considered part of case information processing. After the subject

14 Courtesy of Bart van Swol and Arnie Bosman from the University of Amsterdam, The Netherlands 
had estimated the case probability, a pause screen appeared. Subsequently, the experimenter announced the next disease in the sequence, and started the presentation of the next case.

After finishing the task, subjects were debriefed. The main purposes of the study were explained, and subjects were given an opportunity to ask questions or to make remarks. Finally, they received a reward for participating in the study.

\section{Analysis}

Average reading times for individual case statement, for the Enabling Conditions and Consequences parts of cases, for complete cases, and for the case evaluation questions were computed. To control for differences in length of corresponding case statements, reading times were expressed in milliseconds per word, for individual statements as well as for complete cases. In addition to reading times, the mean percentage estimates for each subject over the cases of the same type were also calculated.

The data on one of the NO cases, i.c., the one in which a patient actually suffering from pernicious anemia, but announced as someone with iron-deficiency anemia, was described, were discarded, as many subjects apparently confused both ailments. Due to occasional disturbances during an experimental session, a small number of reading times of individual statements had to be removed from the analysis; if this had to be done, average reading times of Enabling Conditions and Consequences were computed using the undisturbed reading times of the remaining case statements. For one subject, three cases, and for another one, one case had to be completely removed; hence, their reading times for the particular case types were computed over three instead of four cases. Furthermore, if a "no" answer on the case evaluation question was followed by a percentage estimate higher than 50 , or a "yes" answer on this question was followed by a percentage estimate below 50 , the respective probability estimates were also excluded from the analysis. Some subjects misinterpreted the probability estimation instruction: They apparently evaluated their own answer on the preceding case evaluation question, rather than the condition of the patient with respect to the announced disease (thus, they responded, e.g., " $90 \%$ ", if they were fairly sure their previous "no"-answer was correct).

In summary, for every subject for all case types, reading times were avail able, though in a few instances based on less than four cases of a specific type (PP, AP, PA, AA, or NO); as far as the probability estimates are concerned, the data of two sixth-year students could not be included in the overall within-subjects analysis, because the probability estimates for one case type could not be computed. The data of these subjects were included in those analyses in which the missing case type data were not involved, though.

The procedure described above finally yielded the following measures per subject and per case type (PP, AP, PA, AA and NO):

1) Average reading times for individual statements, for clusters of statements and for complete case descriptions, expressed in milliseconds per word. Reading times for the case evaluation questions are included in the average reading times for the complete case descriptions, as they are considered to reflect additional information processing of scripted information; but these times are also analyzed separately, as case evaluation latencies;

2) Average probability estimates expressed as percentages.

The reading times of complete cases were analyzed in a $2 \times 4$ ANOVA with expertise level as between subjects factor and case type as within subjects factor. The 
NO-cases were not included in this analysis, because we had no preconceived notion about the case processing times for these cases and could not exclude the possibility that they would cloud a possible interaction effect between expertise level and typicality. The case evaluation latencies and the percentage estimates were analyzed in a $2 \times 5$ ANOVA, with expertise level as between-subjects factor and case type as within subjects factor. In this analysis, the NO-cases were included, because they were expected to yield extreme values (i.c., short case evaluation latencies and low probability estimates). In addition, the reading times of the Enabling Conditions and Consequences parts of cases were analyzed in a $2 \times 2 \times 2$ analysis of variance with expertise level as between subjects factor and typicality and illness script component (i.e., Enabling Conditions or Consequences) as within-subjects factors. However, since the experimental setup does not allow for complete separation of Enabling Conditions and Consequences, because the Enabling Conditions always occur first in the case descriptions, reading times of Consequences statements may be influenced by typicality of the preceding Enabling Conditions, while the reverse is not possible. Consequently, an additional $2 \times 2 \times 2$ ANOVA was performed, with expertise level as between subjects factor and typicality of Enabling Conditions and typicality of Consequences as within subject factors; dependent variable in this analysis was the average reading time of the Consequences part of the cases. The aim of this analysis was to investigate the influence of typicality of the preceding Enabling Conditions on reading times of the subsequent Consequences (either typical or atypicall).

Case statements that were either a mixture of Enabling Conditions and Consequences, or consisted of Enabling Conditions occurring after the first Consequence in a case ${ }^{15}$, were not included in the analysis of the reading times of the individual Enabling Conditions and Consequences parts of the cases. Thus, if we refer to reading times for Enabling Conditions separately, only "pure" Enabling Conditions presented before the first Consequence are included.

Finally, reaction times and probabillity estimates for the NO-cases were analyzed separately, as an outside control, and contrasted with PP and AA type cases in separate $2 \times 2$ analyses of variance.

\section{RESULTS}

\section{Case reading times}

Analysis of variance of the average reading times for complete cases, expressed in milliseconds per word, with expertise level as between subjects factor and case type (the NO-cases not included) as within subjects factor, showed a significant main effect of expertise level, $F(1,58)=4.244, p<.05, M S_{\mathrm{e}}=13,648.488$, a significant main effect of case type $F(3,174)=18.556, p<.0001, M S_{\mathrm{e}}=920.194$, but no significant interaction between expertise level and case type. Table 4.1 shows the results.

15 To make case descriptions as natural as possible, some statements consisted of a mixture of Enabling Conditions and Consequences (e.g;, a current complaint which referred to a previous complaint), while sometimes the patient revealed an Enabling Condition at the end of the case (e...., "I've been on holiday to Spain lately"). 


\section{Table 4.1}

Average case reading times (expressed in milliseconds/word) as a function of expertise level and case type.

\begin{tabular}{rcccccc} 
& \multicolumn{6}{c}{ Case Type } \\
\cline { 2 - 6 } Expertise Level & PP & AP & PA & A A & NO & Means \\
\hline Sixth-year Students* & 230 & 240 & 253 & 268 & 246 & 247 \\
Family Physicians* & 190 & 221 & 224 & 232 & 218 & 217 \\
& & & & & & \\
Means & 210 & 230 & 238 & 250 & 232 & 232 \\
\hline
\end{tabular}

$\mathrm{N}=30$

From Table 4.1 it can be read that consistently for all case types, family physicians were on the average faster than sixth-year students; expressed as a percentage, it took these students about $14 \%$ more time to read a case description than family physicians. Furthermore, reading times increase from PP type cases via AP and PA type cases to AA type cases, while the NO type cases, which were not included in this analysis, fall somewhere in-between. These results support at least one important prediction of the illness script theory: Experienced physicians have well formed illness scripts that enable them to process case information faster than sixth-year students; generally, the better the fit between the incoming information and the activated illness script, the faster this new information is processed. Therefore, completely prototypical cases are processed fastest, whereas an increasing degree of atypicality results in longer processing times.

The absence of an interaction between expertise level and case typicality for the reading times of the four case types suggests that typicality has to a large ex tent the same effect for both sixth-year students and family physicians. This finding is not in line with the illness script theory. What we are particullarly interested in is a possible three-way interaction between expertise level, typicality, and illness script component (i.e., Enabling Conditions or Consequences). Hence, a $2 \times 2 \times 2$ ANOVA with expertise level as within subjects factor and typicality and illness script component as between subjects factors was performed.

The results are displayed in Figure 4.1. In addition to the already mentioned significant main effect of expertise level, a significant main effect of illness script component, $F(1,58)=92.548, p<.0001, M S_{\mathrm{e}}=797.538$, a significant main effect of typicality, $F(1,58)=37.067, p<.0001, M S_{\mathrm{e}}=671.971$, and a significant interaction between typicality and illness script component, $F(1,58)=16.332, p<.0005, M S_{\mathrm{e}}=$ 632.876 , were found. The two-way interactions between expertise level and illness script component and between expertise level and typicality, and the three-way interaction between expertise level, typicality, and illness script component, were not significant. Figure 4.1 shows that in general, typical case parts were processed faster than atypical case parts, and Consequences faster than Enabling Conditions. From this figure, it can also be read that the typicality by illness script component interaction can be accounted for by prototypical Consequences being processed relatively fast compared to atypical Consequences, while typicality of 
Enabling Conditions does not seem to exert a large influence on processing speed. However, as the three-way interaction between expertise level, typicality, and illness script component was not sigmificant, it can not be concluded from the data that the typicality by illness script component interaction should be attributed differentially to the two expertise levels: Apart from a general effect of expertise, reading speed of both expertise groups for Enabling Conditions information is scarcely influenced by typicality. Thus, the data do not suggest a processing advantage of expert physicians for prototypical Emabling Conditions.

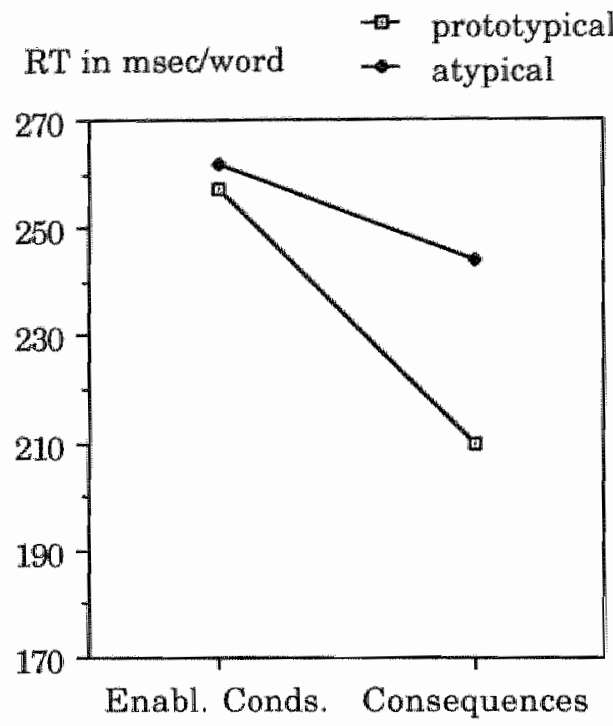

Sixth-year students

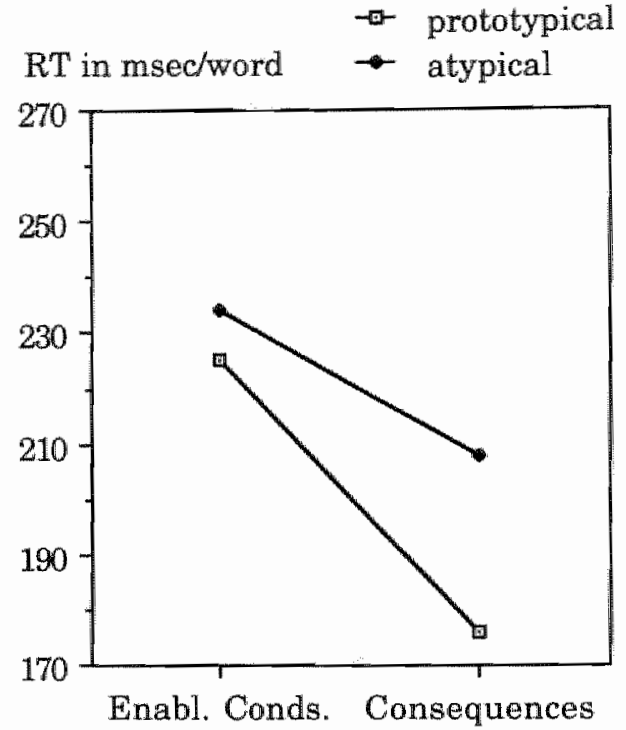

Family physicians

Figure 4.1

Reading times (expressed in msec/word) as a function of typicality of Enabling Conditions and Consequences, for sixth-year students (left hand panel) and for experienced family physicians (right hand panel).

As already mentioned, a problem with the previous analyses may be that the processing of Consequences, whether they are prototypical or atypical, can never be considered completely independent from the processing of the preceding Enabling Conditions. While reading the Consequences described in the case, the subjects already have much information about the patient. It is conceivable that processing of prototypical Consequences is slowed down if the preceding Enabling Conditions are atypical, because subjects are still in the process of trying to instantiate the illness script with the atypical contextual and background information. Conversely, processing of atypical Consequences may be speeded up if the preceding Finabling Conditions are prototypical, because the activated illness script is already partially instantiated. To investigate whether typicality of the En- 
abling Conditions had influenced the processing of the subsequent Consequences, processing times of prototypical Consequences preceded by prototypical Enabling Conditions were compared with processing times of prototypical Consequences preceded by atypical Enabling Conditions, and, similarly, processing times of atypical Consequences were compared for either case of typicality of the preceding Enabling Conditions. Thus, a $2 \times 2 \times 2$ ANOVA was performed on the reading times for the Consequences, with expertise level as between subjects factor and typicality of Enabling Condition and typicality of Consequences as within subject factors.
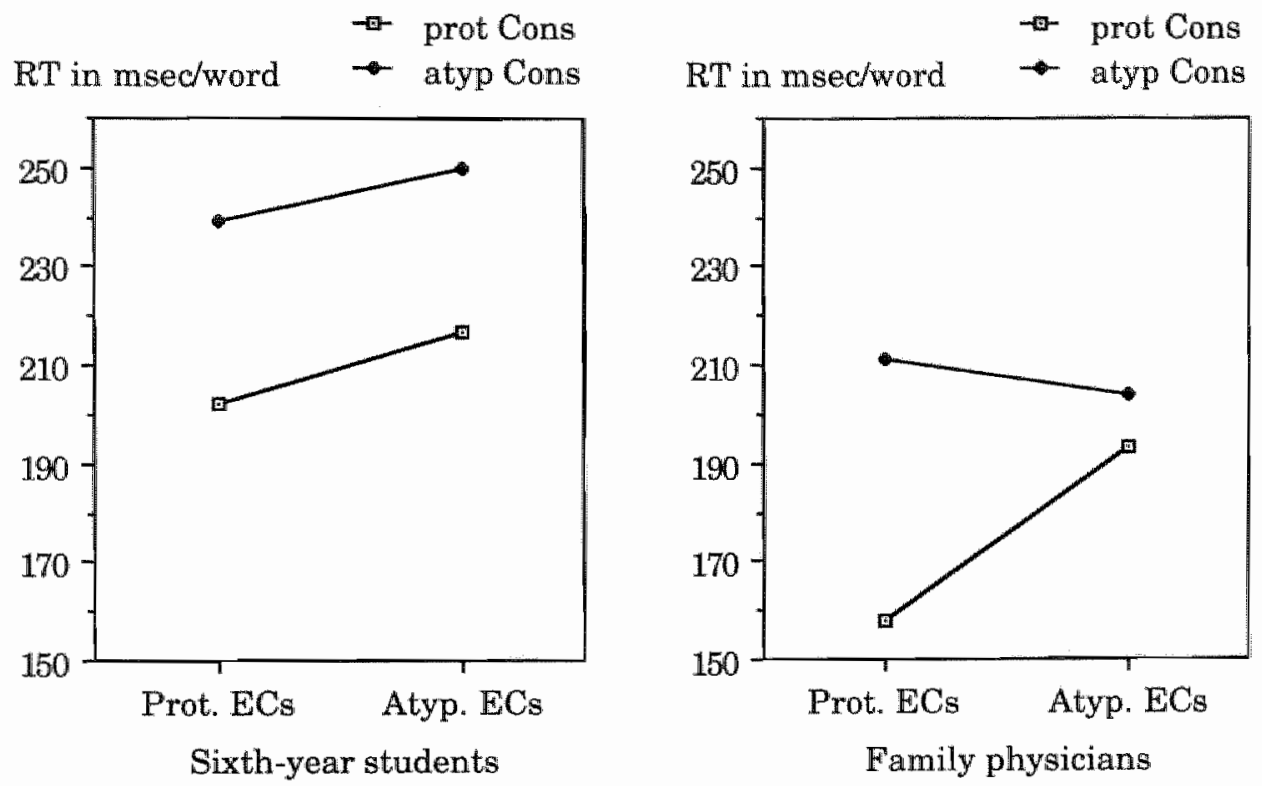

Figure 4.2

Reading times of prototypical and atypical Consequences as a function of expertise level and typicality of the preceding Enabling Conditions and Consequences.

Figure 4.2 shows the results. The analysis revealed the expected main effects of expertise level, $F(1,58)=4.975, p<.05, M S_{e}=15,307.430$, of typicality of Enabling Conditions, $F(1,58)=4.742, p<.05, M S_{e}=11,097.602$, and of typicality of Consequences, $F(1,58)=42.410, p<.0001, M S_{\mathrm{e}}=1,587.712$. In addition, a significant two-way interaction between typicality of Enabling Conditions and Consequences, $F(1,58)=5.559, p<.05, M S_{\mathrm{e}}=1,477.799$, and a borderline significant three-way interaction between expertise level, typicality of Enabling Conditions, and typicality of Consequences, $F(1,58)=3.833, p<.06, M S_{\mathrm{e}}=1477.799$, were found. Inspection of Figure 4.2 shows that reading times of atypical Consequences are relatively independent of the typicality of the preceding Enabling Conditions, while reading times of prototypical Consequences are influenced by typicality of the preceding Enabling Conditions: If the latter are prototypical, subse- 
quent prototypical Consequences are processed particularly fast; the two-way interaction between typicality of Enabling Conditions and Consequences attests to this conclusion. However, comparison of the two panels of Figure 4.2 also shows that this Enabling Conditions by Consequences interaction probably can be attributed completely to the data of the experienced physicians: While for the sixthyear students, the effects of typicality of Enabling Conditions and Consequences seem to be additive, the right-hand panel of Figure 4.2 suggests that experienced physicians show particularly fast processing times for prototypical Consequences preceded by prototypical Enabling Conditions. Therefore, though we did not find evidence for the hypothesis that the influence of typicality of Enabling Conditions and Consequences on reading times is different for subjects at different expertise levels (cf. Figure 4.1), the data do suggest that prototypical Enabling Conditions have an accelerating effect on expert physicians" reading speed of subsequent prototypical Consequences, an effect that is absent for sixth-year students. The speed by which sixth-year students process (prototypical or atypical) Consequences seems to be independent from the nature of the preceding Enabling Conditions.

Finally, the influence of script inconsistent information was investigated by comparing the NO type cases in separate analyses with either completely prototypical, or completely atypical cases. Analysis of variance of reading times of complete cases, with expertise level as between subjects factor and case type (PP versus $\mathrm{NO}$ ) as within subjects factor showed a significant effect of expertise level, $F(1,58)=4.969, p<.05, M S_{e}=6878.022$, and a significant effect of case type, $F(1$, $58)=15.944, p<.0005, M S_{\mathrm{e}}=911.881$, but no significant interaction. Thus, NO type cases are processed significantly slower than completely prototypical cases, by subjects at both expertise levels. Though a similar analysis of variance of AA versus NO type cases showed only a borderline significant effect of expertise level, $F(1,58)=3.48, p<.07, M S e=9093.184$, again a significant effect of case type was found, $F(1,58)=12.304, p<.001, M S_{\mathrm{e}}=793.138$, and no significant interaction. Apparently, NO type cases are processed significantly faster than AA type cases. These results suggest that, on the one hand, deciding to reject a script takes more processing time than just assimilating prototypical, script-fitting information into an already active script, but takes less time than processing of cases for which it remains inconclusive whether or not they are an instantiation of an activated script. Indeed, this conclusion was supported by analyses of the separate Enabling Conditions and Consequences parts of the NO cases: these analyses revealed what might be called a "processing speed gradient:" the Enabling Conditions of these cases were processed with a speed of $278 \mathrm{msec} /$ word, while the Consequences took only $203 \mathrm{msec} /$ word (calculated over both expertise groups). Thus, at the beginning of the $\mathrm{NO}$-cases, the evidently contradictory information takes, probably as a consequence of a surprise effect, relatively much time to be processed, while by the time the first Consequences appear on the screen, subjects may already have decided that the patient described in the case is not suffering from the announced disease. This applies to sixth-year students as well as experts: Besides family physicians having a general speed advantage over their less experienced colleagues, performances of both groups on the NO-cases were highly similar.

\section{Case evaluation latencies (decision times)}

Once faced with the case evaluation question, subjects had to decide as quickly as possible whether the presented case description actually would match some- 
one with the specified disease, or not. As subjects knew the case evaluation question would eventually be presented, it seems reasonable to expect that they tried to decide during the case presentation whether the patient description actually matched the announced illness. It may be expected that for relatively clear-cut cases (i.c., PP cases and NO cases) they woull be able to give a rapid answer to the case evaluation question. For other case types, they might be inclined to ponder some time before opting for a definite answer.

\section{Table 4.2}

Average raw reading times (in milliseconds) for the case evaluation question as a function of expertise level and case type.

Case Type

\begin{tabular}{rcccccc} 
Expertise Level & PP & AP & PA & AA & NO & Means \\
\hline Sixth-year Students* & 4136 & 4401 & 5797 & 6252 & 2488 & 4615 \\
Family Physicians* & 3426 & 4509 & 5069 & 5852 & 2572 & 4286 \\
& & & & & & \\
Means & 3781 & 4455 & 5432 & 6052 & 2530 & 4450 \\
\hline
\end{tabular}

$* \mathrm{~N}=30$

The actual results are displayed in Table 4.2. As differences in length of the case evaluation question were negligible (the only difference being being the length of the name of the illness), raw decision times are presented instead of the decision times in milliseconds per word. The figures in Table 4.2 indicate that this decision generally was not taken too rash. A 2 by 5 analysis of variance of the raw reading times with level of expertise as between subjects factor and case type as within subjects factor revealed no significant main effect of level of expertise, a significant main effect of case type, $F(4,232)=25.007, p<.0001, M S_{e}=$ $4,597,629.911$, but no significant interaction. From Table 4.2 it can be read that, in ascending order, the case evaluation question led to increasing response latencies from $N O$ cases, via $P P, A P$ and $P A$ cases to AA cases, for both expertise levels alike.

A separate comparison between NO type cases and PP type cases revealed that the case evaluation latencies for the former type of cases were indeed significantly shorter than for the latter type, $F(1,58)=20.29, p<.0001, M S_{\mathrm{e}}=$ $2,314,294.047$. This finding also supports the conclusion that by the time the case evaluation question was presented for a $\mathrm{NO}$-case, subjects were already fairly confident that the case was not an instantiation of the activated illness script. It should be noted that, though PP type cases were generally processed faster than No type cases (cf. Table 4.1), the case evaluation latencies of PP type cases were longer than those of the NO type cases (cf. Table 4.2). Apparently, subjects at both expertise levels are more strongly inclined to reject NO-cases, than to accept PPcases. 


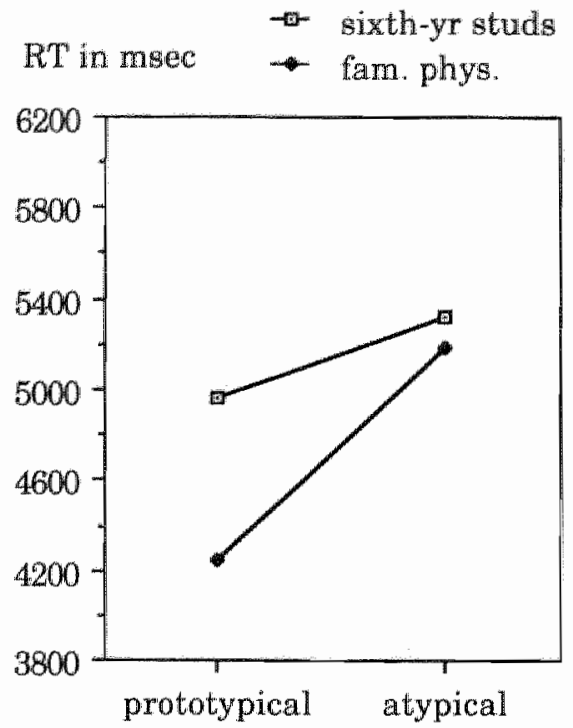

Enabling Conditions
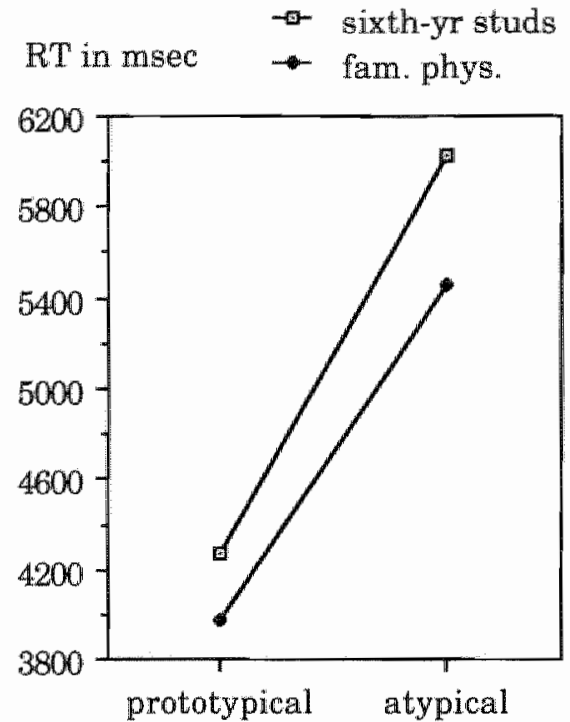

Consequences

Figure 4.3.

Case evaluation latencies (expressed in msec) as a function of typicality and expertise level, for Enabling Conditions (left hand panel) and Consequences (right hand panel).

For the case evaluation latencies, also the possibility of an interaction between expertise level and the influence of typicality of Enabling Conditions was investigated. Since the general hypothesis was that experienced subjects would be more inclined to take Enabling Conditions into consideration, for the case evaluation latencies, it may be expected that it took family physicians relatively more time to answer the case evaluation question if the Enabling Conditions were atypical, than if they were prototypical. For the sixth-year students, on the other hand, differences would be smaller or essentially absent.

However, the expected interaction was not corroborated by the data. Figure 4.3 shows the results. For the Enabling Conditions (left hand panel), a significant main effect of typicality was found, $F(1,58)=4.920, p<.05, M S_{\mathrm{e}}=2548789.171$, but no significant effect of expertise level, nor a significant interaction. This result may be due to a large intersubject variation in decision latencies; inspection of the left hand table of Figure 4.3 surely does not even faintly suggest that the two lines in the graph are parallel. The results of a similar analysis of Consequences are shown in the right hand panel of Figure 4.3. Like in the case of the Enabling Conditions, this analysis also revealed a significant effect of typicality, $F(1,58)=$ $26.003, p<.0001, M S_{e}=3043039.244$, but no effect of expertise level, nor a significant interaction. Figure 4.3 (right hand panel) suggests that subjects at both expertise level are about equally strongly influenced by typicality of Consequences. 
In summary, analysis of the latencies of the case evaluation question showed that subjects are sensitive to manipulations of typicality of case descriptions, but the data did not support the hypothesis that this sensitivity would discriminate between subjects at different expertise levels: Experienced subjects were not more inclined to take Enabling Conditions into consideration than advanced students.

\section{Probability Estimates}

The results of the analysis of the probability estimates are depicted in Table 4.3 and Figure 4.4. Since for some subjects data had to be discarded because of misunderstanding of the probability estimation question, the analyses presented below show some small differences as to the number of subjects included.

\section{Table 4.3}

Average probability estimates (expressed as percentages) as a function of expertise level and case type.

\begin{tabular}{rrrrrrrr} 
& \multicolumn{6}{c}{ Case Type } & \\
\cline { 2 - 6 } Expertise Level & PP & AP & PA & AA & NO & Means \\
\hline Sixth-year Students* & 73.11 & 62.33 & 53.11 & 40.86 & 12.13 & 48.31 \\
Family Physicians\$ & 79.67 & 60.12 & 57.34 & 40.77 & 7.74 & 49.13 \\
& & & & & & \\
Means & 76.50 & 61.19 & 55.30 & 40.81 & 9.86 & 48.73 \\
\hline
\end{tabular}

* $\mathrm{N}=28$

$\S \mathrm{N}=30$

A $2 \times 5$ ANOVA (NO type cases included) of the data in Table 4.3 revealed no significant main effect of expertise level on probability estimations, a significant effect of case type, $F(4,224)=235.961, p<.0001, M S_{e}=156.263$, but no significant interaction between expertise level and case type. The most right-hand column of Table 4.3 shows that both expertise groups did not differ as far as their general in clination to assign probability estimates is concerned. Thus, all differences between experts' and non-experts' probability estimates within the respective columns of Table 4.3 may be considered a consequence of different likelihood of illness script instantiation, based on identical case information, by subjects at different expertise levels. Comparison of the column means of this table shows that manipulating typicality of Enabling Conditions and Consequences had a profound effect on probability estimates: For both expertise levels, the probability esti mates decline from PP type cases via AP, PA., and AA type cases to NO type cases. Thus, subjects generally assigned a high disease probability to the patients described in the PP case variants (about $76.5 \%$ averaged over both levels of expertise), while for the AA case variants these estimates were much lower (about $41 \%$ over both levels of expertise). The AP and PA case types fell somewhere in-between, with mean probability estimates of $61.19 \%$ and $55.30 \%$, respectively. Dis. 
ease probability estimates of the NO cases, averaged over both expertise groups, did not exceed the $10 \%$ level.

A separate comparison between $\mathrm{AA}$ and $\mathrm{NO}$ type cases revealed a significant difference between these two case types, $F(1,57)=150.869, p<.0001, M S_{e}=$ 184.075. Therefore, it can be concluded that even completely atypical cases had a significantly higher likelihood of being considered instantiations of activated illness scripts than contradictory cases; the difference in probability estimates between these two types of cases amounted to $30 \%$ (cf. "Table 4.3).

It was also hypothesized that, for sixth-year students, typicality of Enabling Conditions, unlike typicality of Consequences, would not contribute much to the probability estimates assigned to a case. For experienced physicians, on the other hand, both typicality of Enabling Conditions and typicality of Consequences would influence the probability estimates. it would. Thus, an interaction was expected between typicality of Enabling Conditions and expertise level, with the more experienced group showing sensitivity to typicality of Enabling Conditions, and the less experienced group not, or, if anything, at least not to the same extent. Thus, the data were analyzed by a $2 \times 2$ ANOVA with expertise level as between subjects factor and typicality of Enabling Conditions as within subjects factor. Average percentage estimates of cases with prototypical Enabling Conditions were computed over all cases with this type of Enabling Conditions, regardless of the nature of the following Consequences.
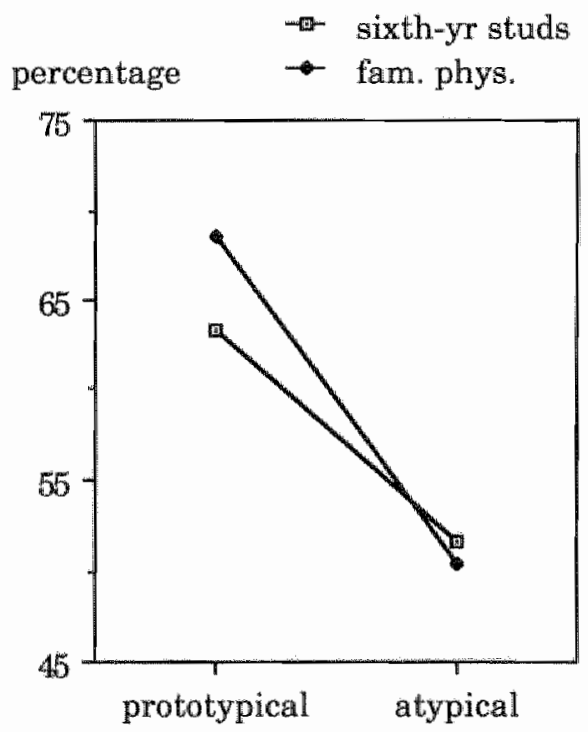

Enabling Conditions
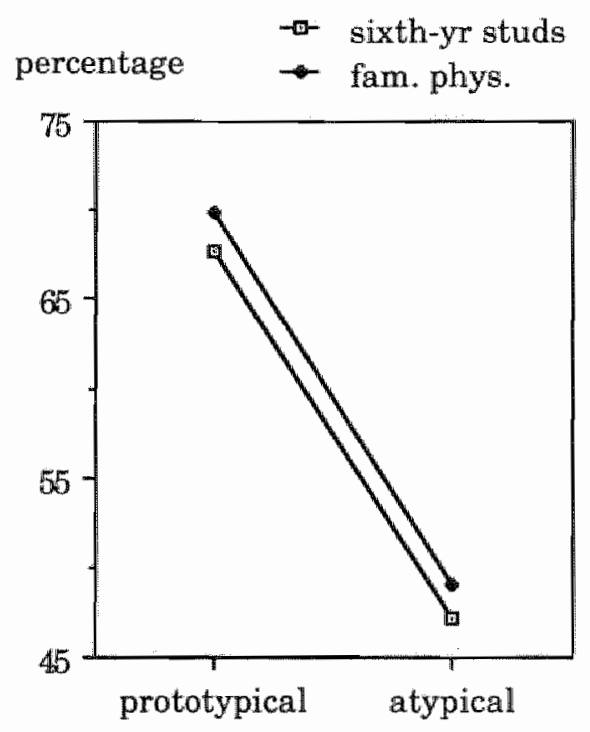

Consequences

Figure 4.4.

Probability estimates as a function of typicality and expertise level, for Enabling Conditions (left hand panel) and Consequences (right hand panel). 
The results showed, apart from the expected main effect of typicality $F(1,57)$ $=97.993, p<.0001, M S_{e}=67.129$, indeed a significant interaction between expertise level and typicality, $F(1,57)=4.301, p<.05, M S_{\mathrm{e}}=67.129$. In Figure 4.4, (lefthand panel) these results are displayed graphically. It can be read from this figure that experienced family physicians generally assigned higher probability estimates to cases with prototypical Enabling Conditions and lower probability estimates to cases with atypical Enabling Conditions, whereas sixth-year students showed less sensitivity to this typicality manipulation, even though it evidently can not be concluded that sixth-year students were altogether insensitive to typicality of Enabling Conditions.

In Figure 4.4 (right hand panel), the results of a similar analysis of Consequences are displayed. Apart from the general typicality effect, $F(1,57)=96.911, p$ $<.0001, M S_{\mathrm{e}}=130.601$, no other significant results were obtained for the Consequences; indeed, visual inspection of Figure 4.4 (right hand panel) easily confirms the outcome of the analysis that typicality of Consequences influenced subjects at both expertise levels to about the same extent. Comparison of the two panels in Figure 4.4 also suggests that typicality of Consequences in general played a slightly more important role in determining probability estimates than typicality of Enabling Conditions, especially so at the level of the sixtli-year students.

Thus, unlike the case reading time data, the results of an analysis of the probability estimates support the hypothesis that experienced family physicians are, more than sixth-year students, inclined to take typicality of Enabling Conditions into consideration in determining how likely a case is an instantiation of a specific illness script.

\section{DISCUSSION}

In the present study, the influence of expertise level and typicality of case information on case processing times and case probability estimates was investigated. In this section, we will elaborate on the implications of the present results for the illness script theory, and especially for the notions about the development of illness scripts expounded in the previous chapters. But first, we may conclude that manipulating typicality of case information turned out to be a powerful experimental tool to investigate processing speed and judgment of medical (clinical) information: Consistently large differences in processing times and probability estimates as a function of case typicality were found. As such, the present study underscores the findings of Bellezza and Bower (1981) and Yekovich and Walker (1986) that experimental manipulation of typicality of stories can be used to investigate script processing characteristics.

As far as processing times are concerned, we found a general effect of expertise, typicality, and illness script component: Experts were faster than non-experts, typical information was processed faster than atypical information, and Consequences were processed faster than Enabling Conditions. However, this latter effect may be due to the fact that in the cases, Consequences always were preceded by Enabling Conditions, and illness script instantiation may have been already partly accomplished by the time subjects arrived at the Consequences information. Though level of expertise exerted a general influence on processing times, none of the interactions in which expertise level featured was significant. Thus, the pattern of case reading times generally did not support the idea that experts' illness scripts are characterized by better integration of Enabling Conditions, because if this were the case, a signifiant three-way interaction between 
expertise level, typicality, and illness script component would have materialized, with experts showing, apart from their general superiority in processing speed, especially fast processing times for both prototypical Enabling Conditions and prototypical Consequences, while students would process prototypical Consequences aignificantly faster than prototypical Enabling Conditions. Rather, the present results suggest that differences in knowledge structure between experts and non-experts are of a quantitative nature; an additional analysis was necessary to reveal a possible interaction between expertise level, typicality, and illness script component: The data suggest that expert physicians, compared to sixthyear students, are particularly fast when reading prototypical Consequences preceded by prototypical Enabling Conditions. Nevertheless, though no support was found for a differential developmental trace of Enabling Conditions and Consequences, the results are in keeping with the notion that the presence of script-like structures can adequately account for the results: Experts, who are assumed to have better developed scripts, process information faster than non-experts, and similarly, prototypical information, that corresponds to expected or default values of script slots, is processed faster than atypical information. As such, this result is in line with earlier studies on processing speed of script related information (e.g., Bellezza and Bower, 1981; Yekovich \& Walker, 1986).

The results also showed that if case descriptions deviate too much from what is feasible given a specific illness script, reading times show a significant drop. Thus, after some initial hesitation, NO cases are processed relatively quickly. Apparently, for this type of cases, less processing is required, probably because already in an early stage of the case presentation, subjects have decided that the activated illness script can not be instantiated by the case information; hence, they have rejected it and do not feel inclined to try to fit incoming information into it. In fact, in the present study, an inverted-U relationship between "script fitting quality" of case information and processing speed was found: Both information that can be easily filled in an activated script and information that evidently contradicts allowed script features or slot values are processed fast, i.e., without elaboration or consideration, while information of intermediate fitting quality requires additional processing time. The long case evaluation latencies for partly and completely atypical cases (cf. Table 4.2) underscores this conclusion.

As far as probability estimates are concerned, also a general effect of case typicality was found. Completely prototypical cases were assigned probability percentages in the $70 \mathrm{~s}$, completely atypical cases in the lower $40 \mathrm{~s}$. Cases with either prototypical Enabling Conditions or prototypical Consequences (but not both) received probability percentages in the range of 55-62\%. Thus, both typicality of Enabling Conditions and typicality of Consequences contributed to the overall subjective likelihood estimate. Separate comparisons revealed that experienced physicians were somewhat more inclined to take prototypicality of Enabling Conditions into consideration than sixth-year students, though subjects at both expertise levels were clearly influenced in their probability estimates by this variable. However, the higher sensitivity of more experienced subjects for contextual and patient background information is in line with the proposed development of illness scripts.

On basis of the present result, no definite conclusions can be drawn concerning the role of illness scripts in diagnostic situations. It should be reminded that Hobus et al. (1987, 1990) found that experienced physicians who had access to Enabling Conditions were able to use this information in activating diagnostic hypotheses, while inexperienced physicians were much less able to do so. The study presented in the previous chapter showed that this phenomenon could not be ac- 
counted for by a mere lack of knowledge of Enabling Conditions from the part of the less experienced subjects. Though the present study suggests that experts may have better illness scripts than non-experts, as evidenced by faster processing times, there are no reasons to assume that integration of Enabling Conditions into illness scripts shows a developmental lag compared to integration of Consequences: Integration of both types of knowledge seems to show a largelyr parallel developmental trace. In summary, the role of Enabling Conditions and Consequences may in many ways be similar for subjects at a specific level of expertise, i.e., experts might also be more able to profit from knowledge of Conse* quences than non-experts. As Hobus et all (1990) did not investigate the influence of knowledge of Consequences on diagnostic hypothesis formation, no definite conclusions can be drawn yet. 



\title{
CHAPTER 5
}

\section{A RECOGNITION STUDY TESTING THE PSYCHOLOGICAL VALIDITY OF ILLNESS SCRIPTS}

\author{
INTRODUCTION
}

Table 5.1

Example of a "Jack story" (adapted from Graesser, Grordon and Sawyer, 1979).

"Jack decided to take his girlfriend, Chris, out for a nice dinner. He called a friend of his who recommended a good restaurant. He then went out to his car and drove over to Chris house to pick her up. They drove to the restaurant and he stopped out in front. They got out of the car and let the valet park it. Then they walked inside and Jack confirmed their reservations with the hostess. They sat for a few minutes in the waiting area, and then the hostess escorted them to their table. After the waitress had introduced herself, they ordered cocktails. They browsed the evening paper for a while, and then looked at their menus. After they decided what they wanted to eat they ordered dinner. As their food was being served, they placed their napkins in their laps. They enjoyed their meal, and had another drink. When they were finished, Jack paid the bill, and left a generous tip. Then they walked out of the restaurant, got their car, and drove home."

Table 5.1. shows a, textually somewhat adapted but otherwise typical, example of an experimental story employed in studies on memory for scripted actions (e.g., Abbott et al., 1985; Bellezza \& Bower, 1981; Graesser et al., 1979; Schank \& Abelson, 1977; Smith \& Graesser, 1981; Walker \& Yekovich, 1984; Yekovich \& Walker, 1986). This type of stories is generally about a fictitious character (usually a "Jack", "John", or "Mary"), performing a scripted activity, like going to a res"taurant, visiting a dentist, getting up in the morning, or flying by plane to another city. The example in Table 5.1 is a highly typical instantiation of the "restaurant script"; as such, the story is neither very informative, nor very interesting; it may even be considered a bit boring. Consequently, it may come as no surprise that subjects who are, after some time lapse, asked to answer questions about this story (e.g., "Did the hostess hand the menu to the customers?" "Did Jack order an appetizer?", "Did the couple use napkins?", "Did the guests have a conversa. tion?") have no specific memory representation for this text, but tend to rely, in deciding on an answer, on their general scripted knowledge about what is usually going on in a restaurant. Bartlett (1932/1954) already reports that the memories people have of stories tend to become increasingly script-like with longer retention intervals; studies from the late $1970 \mathrm{~s}$ onwards have provided a theoretical basig for this and related memory phenomena (e.g., Abelson, 1981; Bellezza \& Bower, 1981; Bower et al., 1979; Graesser et al., 1979; Schank \& Abelson, 1977; Walker \& Yekovich, 1987). 
In all these studies, scripts are viewed as high-level, integrated memory structures. As such, scripts fit quite well in memory representations based on associative networks (cf. Anderson, 1983; Sharkey \& Mitchell, 1985; Walker and Yekovich, 1987; Yekovich \& Walker, 1986), which consist of a number of nodes, interrelated by links. The nodes in such a network may represent objects, ideas, or actions in the script. If a script is activated, this activation spreads throughout the network by way of the interconnecting links. Each node in the script is activated, with the amount of activation dependent on the number of links the particular node has with other nodes, and the strength or weight of these links. Important nodes, that are central to the script, have many links with other nodes and hence, are strongly activated, while less important or less central nodes have few links and receive only weak activation as a consequence of the activation of the script. For example, in an activated restaurant-script, the ordering and eating of food and subsequent payment for it are highly central nodes, while information about drinks or people having a conversation is less central, though it certainly fits in this script.

In addition to this activation of script concepts as a consequence of spreading activation in an associative memory sub-network - called internal or implicit activation - concepts can also become active as a consequence of external activation. The source of this external activation is usually an input text. Concepts and actions that are only weakly activated by virtue of spreading activation, because they are not highly central to the script, may receive direct external activation and consequently become strongly activated. In fact, this is part of the process of script instantiation (cf. Schank \& Abelson, 1977), i.e., the filling-in of script slots and the substitution of default script variable values by incoming information. As far as the "Jack story" in Table 5.1 concerns, readers may only become aware of the fact that napkins are usually present in a restaurant situation, when they are explicitly mentioned. On the other hand, the fact that information about napkins being placed in laps can be easily assimilated when a restaurant script is activated, indicates that the concept "napkin" is at least weakly activated, for most readers would be surprised if Jack and his girlfriend placed a physically similar object, for example, a floor-cloth, in their laps during dinner. In fact, events, actions or concepts that appear in a text but are not part of the regular script structure, such as using a floor-cloth as napkin in a restaurant scene, are represented separately, as tags to the instantiated script. Thus, tags are deviations from the "mass of schema-relevant information" (cf. Graesser, 1981). Consequently, the eventual memory representation of a scripted event consists of an activated script and a number of tags, with the tags being instantiation-specific. This idea has been elaborated in a "script pointer plus tag" model (Graesser et al., 1980) and a "schema copy plus tag" model (Graesser \& Nakamura, 1982); both models are reminiscent of the "schema with correction" notion endorsed by Bartlett (1932/1954) and Woodworth (1958).

The "script pointer plus tag" and "schema copy plus tag" models are more specific alaborations of the general approach of memory for instantiated scripts as a composite memory trace (Bower et al., 1979; Graesser et al., 1979; Sanford \& Gerrod, 1981; Walker \& Yekovich, 1987; Yekovich \& Walker, 1986). This composite trace consists of the activated script and the memory representation of the tags attached to it While the scripts themselves are stable memory structures, the trace of items tagged to activated scripts is of a more ephemeral nature: With increasing time lapse between the original instantiation and a subsequent reminding of the instantiated script, tags are apt to decay, particularly if the information they contain is less relevant or salient, or if it concerns surface features of the original 
input. For example, it is likely that if a reader of the "Jack story" presented in Table 5.1 formed a tag for the information that the customers placed napkins in their lap, this tag quickly decays; had the eating couple instead used a floor-cloth, then the corresponding tag would probably be granted a longer life. Eventually, only the script itself, and perhaps a few highly salient or relevant tagged items, remain. In a more recent modification of the script theory, Schank (1985) makes a distinction between generalized events stored in situational memory, and the unusual or interesting parts of individual events that remain in event memory; thus, reminding is in his vision dependent on two memory traces as well.

In summary, the representation of instantiated scripts can be conceptualized as a composite memory trace, consisting of implicitly activated central script concepts, and explicitly activated peripheral or atypical actions and concepts. This is the view of both Yekovich and Walker (Walker \& Yekovich, 1984; Yekovich \& Walker, 1985, 1987) and Graesser and his colleagues (Graesser \& Nakamura, 1982; Graesser, et al., 1980; Nakamura \& Graesser, 1985; Smith \& Graesser, 1981), though these latter authors make no specific assumptions about the underlying memory representation of scripts. In both approaches, a minimum of activation is necessary to insert information into the memory trace, while beyond a certain activation level no additional benefit might be expected; however, the models do not explicitly preclude that stated script-typical information may also be tagged, and thus have a memory advantage compared to unmentioned typical information 16 .

Central aspect of most script related studies has been the effect of type of information on recall and recognition memory parameters. Usually, two types of information are distinguished: script typical and script atypical information. As a consequence of the fact that scripts have a goal structure, some studies do not distinguish between typical and atypical information, but between central and peripheral, or between necessary and accidental, or between expected and unexpected, information. Though these dimensions are not identical (e.g., placing a napkin in one's lap may be typical, but not highly central, in a restaurant script), the way they are actually applied in the studies renders them comparable; for example, atypical items may be defined as peripheral items with a certain degree of saliency. Furthermore, Graesser et al. (1979) provide empirical evidence that in a script context, typicality and necessity are closely related dimensions; they report a correlation of 92 between typicality and necessity ratings of scripted actions. Thus, in the present study, we will employ the "typical-atypical" dimension to cover the whole typicality-centrality-relevance-necessity complex described above.

Generally, the following line of reasoning is employed to derive memory performance hypotheses from the composite memory trace model of scripted events. Unstated script typical information is assumed to be implicitly activated and inserted into the memory trace upon script activation, while script atypical information has to be explicitly presented to become part of that trace, in the form of a tag. Unstated atypical information will not be activated, and hence not become part of the trace anyway. Stated typical information may be tagged, but the memory of this tag will easily be lost, because it does not add anything to the already implicitly activated typical information. As a corollary, in a reminding situation

16 Though Graesser et al. (1979) expect "zero memory discrimination" for highly typical scripted. information (i.e., that subjects perform at chance level in determining whether this information was actually mentioned in a text), they admit that retention of wording and surface features may influence memory, especially after short retention intervals. 
(i.e, recall or recognition), memory performances for typical and atypical information will be different. The most salient aspect of this prediction is that it will be difficult for subjects to remember whether script typical information has actually been presented, or was merely implicitly inferred, as a consequence of activation of the script. Returning to the "Jack-story" in Table 5.1, tt may be difficult for subjects to remember whether it was explicitly stated that Jack made a call for a reservation, that the young couple waited in the waiting area, that the hostess handed the menu, or that Jack paid the bill. (Indeed, in answering the second question, many readers may not even notice that in the text neither the age of the participants, nor the duration of their relationship was referred to). In a recognition task, subjects will be inclined to answer these questions on basis of their scripted knowledge, rather than on basis of the actual text; if asked to recall the story, subjects will also use this knowledge, and consequently will probably recall that Jack called for a reservation, that the couple sat down at a table, and so on. However, the fact that they browsed the evening paper before ordering food might be correctly recognized and recalled, because this action is not very typical for the restaurant script. Similarly, Europeans might also be expected to have good memory for the information that the valet parked the car, because this is not a typical action in the "European" restaurant script.

In summary, typical script information will show a high number of recall intrusions (i.e., information present in recall, but not in the input text) and recognition false alarms (i.e., unmentioned items being positively recognized), whereas atypical script information will not. Empirical evidence has been found that supports the hypothesis that both recall intrusions and recognition false alarms are higher for typical than for atypical information. For example, Bower et al. (1979), Graesser et al. (1980), Maki, (1990), and Smith and Graesser (1981), found that subjects are prone to "recall" typical or central script activities that were never explicitly presented. With increasing retention time interval, this effect may become even more pronounced: Recall becomes increasingly script-guided, because the atypical tagged actions gradually decay from memory, while the general script remains intact, and subjects tend to rely more and more on it during recall (Graesser \& Nakamura, 1982; Graesser et al., 1980; Smith \& Graesser, 1981).

In addition to the false alarm rate is the hit rate (i.e., the proportion of actually presented items being positively recognized) an important facet of recognition memory performance. Recognition memory discrimination is said to be good if hit rates are high and false alarm rates are low. Script based studies have quite consistently found better recognition memory discrimination for atypical than for typical information (Bellezza \& Bower, 1981; Bower et al., 1979; Graesser et al., 1979; Maki, 1990; Nakamura \& Graesser, 1985; Walker \& Yekovich, 1984). Though the recognition advantage of atypical information decreases over time, even after retention intervals of weeks atypical information still shows better memory discrimination than typical information (Smith \& Graesser, 1981). Though this finding is generally agreed upon, it is not yet a foregone conclusion whether individual hit rates are actually higher for atypical items (e.g., Graesser \& Nakamura, 1982) or for typical items (e.g., Graesser et al., 1979; Yekovich \& Walker, 1986). Furthermore, even for atypical actions and concepts, a false alarm rate differing substantially from zero may be found: Graesser et al. (1979) report $16 \%$ false positive responses, even for unmentioned, atypical actions.

Besides hit rates and false alarms, reaction times are also employed as recognition memory parameters. Generally, it is assumed that subjects pass through some information processing stages in determining their recognition re- 
sponse for a particular test item (cf. Bower et al., 1979; Graesser at al., 1979; Walker \& Yekovich, 1984). Though the exact sequence of these stages remains to be determined, searching the memory trace for a tag and judging the likelihood or script relatedness of an item, are two important aspects in the recognition process. The reaction time is thought to reflect the number and duration of stages necessary to determine the recognition response. For example, atypical unstated information can be quickly rejected, i.e., recognized as "not presented", because it is neither likely given the script, nor tagged to the memory trace. For example, with respect to the "Jack story" presented in Table 5.1, subjects are usually able to answer questions like "Did Jack quarrel with the waitress about the bill?" quickly and accurately, because such an action is neither highly typical for a restaurant situation, nor mentioned in the story. Response latencies for script typical, but unstated information, on the other hand, are relatively long; it takes subjects some time to correctly answer questions like "Did Jack make a call for a reservation?", because this is a script typical action, and hence can not immediately be rejected. Subjects have to decide that there is no tag or specific memory trace for this information, and this takes time. In many cases, activation of this kind of information may be sufficiently high to elicit a quick false alarm response. Findings by Nakamura and Graesser (1985) and Yekovich and Walker (1986) confirm these hypotheses. According to these authors, if subjects have to decide on the actual textual presence of script typical, but in fact unmentioned, information, they will give either a quick false alarm response, or a slow correct rejection response. In this latter case, subjects have to resolve a conflicting response tendency: The concept or action that has to be judged is strongly activated, but they are unable to find a memory tag for it. In order to decide on the actual presence of such actions and concepts, subjects will probably try to reinstate the text base, with the objective of finding vestiges of an already largely decayed tag; this process takes time. In case of false alarm responses, on the other hand, subjects do not search for a tag, but decide "immediately" on basis of a high level of activation of the unstated, typical information.

For example, Yekovich and Walker (1986) found hit rate reaction times of 889 and 1093 msec for script peripheral and script central presented items, respectively, while correct rejection times for script central unmentioned items were about twice as long (2032 msec). However, response latencies for false alarms (1124 msec) were not silgnificantly longer than those for hits. These results suggest that false alarms are not a consequence of uncertainty (i.e., guessing that a tag was present) after thorough examination of the memory trace, but based on a subject's unjustified conviction that the item in question has indeed been actually presented in the scripted text. According to the model, this conviction is the result of a high activation level of the concept or action, due to implicit activation" if this level is sufficiently high, a false alarm response may be "immediately" given, while the stage in which the memory trace is searched for a tag, may be entirely sipiped.

The findings of poor memory discrimination and longer recognition reaction times for typical than for atypical information discussed above seem to be relativeIy robust. However, the materials used in the reviewed studies all involved the "classical" Schank and Abelson (1977) type of scripts, i.e., routine everyday situations like going to a restaurant or visiting the dentist. Is there any reason to expect that memory phenomena found for these routine situations will also be found in more complex domains, like medicine? In other words, will the memory phenomena found for classical scripts also be found for the presumed illness scripts? We are not familiar with any study in which this issue is directly addressed, but 
some studies which investigated memory performance in complex real life domains guggest that schema or script-like structures may indeed guide recall or recognition in these domains. For example, Arkes and Harkness (1980) found evidence that diagnosis-consistent, but unstated symptoms, were often falsely recognized by diagnosticians, provided that the diagnosis was known to the subjects. This was confirmed for a medical condition, i.c., Down's syndrome, and a nonmedical condition, the latter one being a malfunctioning plumbing system. These authors conclude that subjects do not just encode the original symptoms, but invoke a schema or prototype when making a diagnosis. In a subsequent recall or recognition situation, this schema is reinstated, with recall intrusions and false recognitions as a result. In a similar vein, Carlson and Dulany (1988) report that subjects may falsely recognize incriminating cues and forget discharging cues in fictitious murder stories, if they are convinced that a particular person has committed the crime. In such a case, the culprit and the actions and motives attributed to him or her may function as a schema. In a study focused on physicians" autobiographical memories, Hassebrock and Prietula (1990) found that subjects recalled clinical findings very poorly, and that those symptoms that they actually recollected were either general inferences from pathophysiological conditions, or salient findings consistent with the diagnosis. However, case features that deviated from the general script, but were critical for establishing a diagnosis or installing a treatment, were also well represented in the protocols; this finding supports the idea that important atypical information is tagged to the script. Irrelevant contextual details were also often mentioned. In general, the results of the Hassebrock and Prietula (1990) study are in line with those of recall studies of scripted stories employing long retention intervals (e.g., Smith \& Graesser, 1981). In summary, though relevant findings are few and far between, and in none of these studies reaction times were used, there is some ground to expect that illness scripts will show similar memory effects as classical scripts.

Table 5.2 gives an impression of what a "Jack story", transferred to a medical situation, might look like. This story also contains typical and atypical elements for the disease in question, i.e., a kidney stones colic. For example, the description of the type and location of the pain (convulsive, unilateral, in the abdomen) and the history of previous treatment for kidney stones, are highly typical; the broken leg six years ago, and the current slight fever, are atypical. However, subjects who have insufficient knowledge about this medical condition (i.e., do not have the appropriate illness script), may not be able to recognize the difference in typicality between the information elements in the story. Consequently, the question crops up whether memory performance can be used to investigate differences between the scripts of experienced physicians and less expert subjects, like medical students. To our knowledge, the effect of expertise level is not studied in any script related research. However, the studies described in the previous chapters of this thesis suggest that (advanced) students' illness scripts are more diffuse, with links between concepts less well established, and with the appropriate values to fill in slots less well circumscribed, than physicians" illness scripts. Thus, it might be expected that implicit activation of typical script concepts will play a less prominent role in non-expert subjects, who will rely more on the explicit presence of information in a medical text. The present study also addresses the issue whether experienced subjects show more script consistent memory performances than relatively inexperienced subjects.

In summary, there is circumstantial evidence that illness scripts may function in a fashion similar to regular scripts, at least as far as memory performance is concerned. In order to investigate whether this is indeed the case, we 
designed an experiment in which some hypotheses, in line with those of the script-based studies described above, were tested for illness scripts. As the previous studies involving complex domain scripts are mostly recall studies, we decided instead to conduct a recognition memory performance study: First, recognition scores are usually considered to be more sensitive memory parameters than recall scores (cf. Graesser, 1981); second, as recognition responses are less sensitive to response editing than recall measures, they may yield a somewhat more veridical picture, and third, in a recognition study, both memory discrimination and reaction times can be investigated, while this latter variable is not very informative in a recall study. In addition, we only investigated recognition memory for short retention intervals, i.e, in magnitude of minutes, not days or weeks. Though a practical concern formed the basis of this design feature (e.g., it is very hard to standardize a time interval for visiting practicing family physicians twice), it should also be emphasized that the results of the script related research described above suggest that the difference in recognition scores between typical and atypical actions and concepts decreases with increasing time interval (cf. Nakamura \& Graesser, 1985); thus, our chances to find an effect would be optimized by taking a short retention interval.

Table 5.2

Example of an illness script story.

"You are just about to have dinner, when you receive a telephone call from Mrs. Jones. She wants you to come immediately, because her husband "is having it again": he is rolling across the room because of the pain, and has vomited several times. Mr. Jones is a 47-year old store-keeper, who is married and has three teenage children. At age 30 , he was treated for bronchitis. Six years ago, he had his leg broken as a consequence of a car accident. Four years ago, he was treated with medicaments for kidney stones. His older brother is known with coronary disease, while his father died at age 58 from a CVA. His 52-year old sister has diabetes mellitus.

When you arrive at the Jones' home, Mr.Jones is sitting on the sofa, smoking a cigarette and recovering a bit; the pain has just subsided. He complains about having had a convulsive abdominal pain at the left side, just abreast of the navel; the pain extended to his groins. The pain emerged all of a sudden, and then gradually subsided; during the attack, he almost couldn't stand it. Earlier that day, he had remarked that his urine showed a red. hue, but he had not paid much attention to it, because he had no pain at that time. His wife, who has measured his temperature, says he has $38.2^{\circ}$ Centigrade fever".

The following hypotheses were experimentally tested:

1) Memory discrimination will be better for atypical items than for prototypical items. Thus, am interaction will be found between typicality and textual presence of items: Unstated prototypical items will tend to be falsely recognized, while unstated atypical items will be correctly rejected. As indicated above, previous script related studies do not consistently predict whether hit rates are higher for stated prototypical or stated atypical items; thus, we have no specific assumptions with respect to the difference between these scores. 
2) This interaction between typicality and textual presence will depend, at least to a certain extent, on level of expertise: For less experienced subjects, whose illness scripts are not yet fully developed, it will be less pronounced than for more experienced subjects. Thus, we predict that a three-way interaction between expertise level, typicality, and textual presence will be found, with the size of the two-way interaction effect of typicality and textual presence being moderated by the expertise level of the subjects included in the analysis.

3) As far as the reaction times are concerned, based on the results of the study described in the previous chapter, a positive relationship between expertise level and response speed will be expected: More experienced subjects will generally take less time to respond than less experienced subjects.

4) The results of Nakamura and Graesser (1985) and Yekovich and Walker (1986) will be corroborated: Reaction times for atypical items will, right down the line, be shorter than for prototypical items. In addition, an interaction will be found between textual presence and typicality: Especially unstated prototypical items will show long reaction times, due to large response latencies for correct rejections.

5) This interaction between typicality and textual presence on reaction times will also be moderated by expertise level: it will be more pronounced for experienced subjects than for inexperienced subjects, whose illness scripts are still in the process of development. Thus, a three-way interaction between expertise level, typicality, and textual presence will be expected, with a full-blown interaction for the experts, and more of an additive effect for the least experienced subjects.

6) The hypotheses concerning reaction times described up till now do not distinguish between different types of responses (e.g., hits versus misses, false alarms versus correct rejections). In order to corroborate the findings of Yekovich and Walker (1986), response times will be computed separately for prototypical and atypical hits, and for prototypical correct rejections and false alarms. It is hypothesized that atypical hits will be associated with fast reaction times, whereas correct rejections will show the largest response latencies. It will be expected that reaction times for prototypical hits and false alarms fall somewhere in-between.

In addition to these hypotheses, two other predictions are investigated:

7) Information that is inconsistent 17 (i.e., contradictory) with the currently activated illness script will be rejected quickly and correctly, faster and more accurately even than unstated atypical information. As far as the example in Table 5.2 is concerned, subjects will be able to quickly and correctly answer questions like "Did the patient appear at the office hours?", because this is not only highly unlikely, given that the patient has a kidney stones colic, but also contradictory to the content of the text. The underlying assumption is that, whereas atypical information may not be activated, script inconsistent information may even be actively inhibited by associative links in the network (i.e. the information "phone call" and "asking to come immediately" may inhibit the concept "office hours"). As the script-inconsistent information in the present study always implicitly or explicitly contradicts one or more statements in the case description, low percentages of "yes" answers and short reaction.

17 We use the word "inconsistent" in a different sense here than it is usually conceived of in script-rellated research: whereas "inconsistent" is often defined as "(temporarily) blocking the progress of the script," we use it in the sense "contradictory to some highly prototypical script slot value." 
times will be expected for this type of items. Though we have of course no reason to exclude that this effect may be stronger for experienced physicians than for advanced students, we do not apriori expect a significant expertise effect: Students may have sufficient knowledge of the diseases to recognize inconsistent information.

8) Two types of unstated prototypical Consequences were distinguished: omissions, i.e., prototypical information that was deliberately omitted or deleted from a case, and paraphrases, i.e, prototypical information that was actually present in a case, but phrased in completely different wordings. For example, statements like "The pain radiated to his crotch," and "he has a slight fever" are paraphrases of information present in the text displayed in Table 5.2, while statements like "When the doctor arrives, he doesn't appear very ill," and "He has had the same kind of attack, a few years ago" are omissions, i.e., they are in line with the kidney stones colic script, but not mentioned. In the script studies discussed in this section, unstated information refers to what we call omissions; we know of no script based study in which the recognition ef fects of paraphrased information are studied. Nevertheless, we expect that both types of statements, being prototypical, will be falsely recognized more often than atypical information, with the paraphrases being even more prone to memory errors, because this latter type of information not only receives script based implicit activation, but the relevant concepts may also be explicitly activated by the input text, though the surface level representation may be different. It is also expected that experienced physicians, as a consequence of having stronger links between illness script concepts in memory, will be more inclined to falsely recognize omissions than student subjects, or will at least. show longer reaction times for this type of information.

By distinguishing two types of patient information, i.c. Enabling Conditions and Consequences, it was also investigated whether there were differences in memory performance between these two illness script components. As the results of the studies in the previous chapters suggest that the integration of Enabling Conditions into illness script structures occurs relatively late in the development of illness scripts, it might be expected that Consequences show script-like memory performance properties in an earlier stage of medical expertise development than Enabling Conditions. In other words, the expected three-way interaction between typicality, textual presence, and expertise level will be moderated by script component: The interaction patterns for experienced subjects will be the same for both script components (i.e., significant two-way interactions between typicality and textual presence), while the less experienced subjects will exhibit patterns similar to those for experts on Consequences, but not on Enabling Conditions. As a consequence, it was predicted that a four-way interaction between expertise level, typicality, textual presence, and illness script component would be found.

\section{METHOD}

\section{Subjects}

Subjects were 22 fourth-year students, 20 sixth-year students, and 23 experienced family physicians. The forth-year students had followed a four year curriculum of theoretical and practical medical education, but they had virtually no clinical experience. They were tested at the end of the term. The sixth-year stu- 
dents had completed at least $75 \%$ of their clerkships, and therefore had walked the wards for 16 months or more. All student subjects were from University of Limburg at Maastricht, The Netherlands.

The experienced physicians were recruited from general practitioners in the Maastricht area. They had on the average 16.25 years experience as practicing family physicians, ranging from 5.75 years to 41 years.

\section{Material}

From the set of 24 diseases used in the study described in the previous chapter, nine were selected to be included in the present study. The criterion for inclugion of a disease was that a case description of a certain length, with sufficient variation in Enabling Conditions and Consequences, could be constructed. The selection also aimed at preserving a maximum amount of variation in seriousness, frequency of occurrence, and tracts between the diseases. The eventual selection consisted of: ameurysm of the aortic artery, herpes zoster, nervous abdominal pain, dermatitis peri-oralis, pre-infaret syndrome, vaginal candidiosis, epidural hematoma, kidney stones colic, and carcinoma of the head of the pancreas. Based on these afflictions, computerized case descriptions were constructed. Each case consisted of a number of statements, ranging from 15 to 24 , in which information about the patient's context and background, the setting (e.g., office hour, emergency telephone call, house call), the main complaints, and some symptoms, was provided. Though each case described a quite "textbook-like" patient, also some atypical patient characteristics and symptoms were included. Appendix 5.A shows an example of a case description.

Table 5.3

Organization of the twelve test statements for each case.

prototypical

stated

1 Enabling Condition

2 Consequences atypical

inconsistent
1. Enabling Condition
1. Consequence
1 Enabling Condition

1 Consequence
1 Enabling Condition

1 Consequence

For each case, a set of twelve test statements was constructed. Five of these statements were exact copies of statements that appeared in the case description. The remaining seven statements differed, at least in wording, substantially from any statement that appeared in the case. These two types of statements are called "stated" and "unstated", respectively; the underlying dimension is referred to as "textual presence." The five stated items and five of the seven unstated items were further divided into three prototypical items and two atypical items (see Table 5.3). The prototypical items contained information that was completely typical for the disease the case description was based upon, while the atypical items contained 
information that was less typical, though not highly unlikely or improbable, for this disease. Like in the previous study, typicality of case information was deter" mined by the answers of physicians in the free production task described in Chapter 3 . The remaining two unstated items contained information that was inconsistent with the case.

Of the twelve test statements, five contained information about Enabling Conditions and seven about Consequences. Table 5.3 shows the complete organization of the set of test statements. From this table, it can be read that the twelve test statements varied on three dimensions: typicality (prototypical, atypical, and inconsistent), textual presence (stated and unstated), and illness script component (Enabling Conditions and Consequences). Appendix 5.B shows the twelve test statements for the case example provided in Appendix 5.A. The two prototypical, unstated Consequences were further divided into one paraphrase, i.e., a complaint, sign or symptom that actually appeared in the case but was expressed in different wordings in the test statement, and one omission, i.e., a Consequence that was completely prototypical for the disease in question, but that had been deliberately omitted from the case description. As it turned out to be difficult to construct for every case both an omitted and a paraphrased Enabling Condition, separate paraphrases and omissions were only designed for Consequences, not for Enabling Conditions. If in the remainder of the present chapter is referred to "prototypical unstated Consequences" without additional qualification, the average value for paraphrases and omissions is meant.

As already indicated, the task consisted of nine cases; the first case, based on the "rupturing aortic aneurysm", was used for practice purposes only. For the remaining eight experimental cases, two different sequences were constructed, to control for possible order effects. All nine cases consisted of a number of text lines that were presented successively on a computer screen; the whole sequence of nine cases formed a block. The nine sets of twelve test statements together also were presented as a block. A press-button device, attached to the computer, was used to record the subjects' responses. The main presentation was written in Authorware; response times were recorded with an accuracy of 1 msec by the experimentation program PET.

\section{Procedure}

Subjects were tested individually, the students at the university department, the family physicians in their own office. Each experimental session consisted of a study phase, an interim task, and a test phase. Both study task and test task were presented on a Macintosh Plus computer screen.

Study phase. After a short general introduction, the study task was started. The nine case descriptions were presented successively. Before each case was started, the name of the disease associated to that case was displayed on the screen, in order to activate the appropriate illness script, and to avoid making it a diagnostic task. If subjects were unfamiliar with or in doubt about the disease, they could ask the experimenter for clarification. If everything was clear, the case presentation was started by pressing a button on the keyboard. Upon starting the case, the statements successively appeared on the screen. Each statement remained visible for a fixed time. This display duration $\mathrm{t}$ (in $\mathrm{msec}$ ) was determined by the formula:

$$
t=1500+(35 * \text { number of text characters })
$$


This display time was constant over all presentations, and based on the reading times results of the study described in Chapter 4 . The size of the base rate of 1500 milliseconds was determined empirically in a pilot study, and it resulted in a display time for each statement that was sufficient for all subjects to read and comprehend the content of the statement, but not long enough to memorize it thoroughly.

Subjects were instructed to read each case as attentively as possible and to try to assimilate as much of the presented information as they could. Though all nine cases were presented successively as a block, after completing each case presentation, an opportunity for remarks, questions, or a short pause was provided. Subjects were not allowed to write anything down. Although it was explicitly announced in the introduction that a test task based on the cases was to be expected, the true nature of this task was not yet revealed to the subjects.

Interim task. After finishing the study phase, a short interim task was presented, in which subjects were interviewed, in a relatively unstructured fashion, about medical journals they were familiar with. This interim task, which lasted for about 3-4 minutes, and for which no data were recorded, was not related to any of the other tasks; its role was simply to clear the subjects' immediate memory for the study task.

Test phase. Next, the test task was administered. For each case, subjects were shown the twelve test statements, one by one. The set of test statements associated to each individual case was always presented as a block; each block of twelve test statements was preceded by the presentation of the name of the underlying disease on the screen, in order to enable subjects to reinstantiate the appropriate illness script. The blocks of test statements appeared in the same order as the cases in the study phase; consequently, the first block of twelve test statements always concerned the practice case, while for the remaining eight blocks, two different sequences were constructed, corresponding to the two sequences of cases employed in the study phase. The order of the individual test statements within each block was randomly determined, for each block individually, during the construction of the experiment, but remained fixed across the actual presentations.

Subjects were instructed to decide as accurately and as quickly as possible for each individual statement whether it had been literally stated in the original case presentation or not. In order to answer this question, the press-button device, until that moment carefully hidden from subjects' view, was connected to the computer. Subjects were instructed to press the "yes" button if they judged a particular test item as having been literally stated in the case, and "no" if they judged that it had not been stated.

It was emphasized in the instruction that the test statements had either been literally stated in the associated case, or differed considerably from any statement in any case, at least in wording. However, it was also stressed that a particular test statement could be very similar in meaning to an item in the associated case, but that meaning should not be taken into consideration: the subject's task was only to judge the literal presence of the statements.

After each block of twelve test statements associated to a case, an opportunity for questions or a short pause was provided. Unlike the study phase, the speed of test phase was subject-paced; test items remained on the screen until a response was emitted. Though accuracy in fact prevailed over speed, subjects were encouraged to respond as fast as they could, while sustaining a maximum level of atten- 
tion. Every time the subject pressed a button, the nature of the response ("yes" or "no") and the reaction time in milliseconds were recorded.

After the test statements for all cases had been presented, subjects were debriefed, and again given an opportunity to ask questions. Finally, they received a small reward for their participation in the study.

\section{Analysis}

For the eight experimental cases and for each subject, the total number of "yes" answers (referred to as "positive recognitions" in the remainder of this chapter) for each type of test statement was computed. This procedure yielded 12 scores on a nine-point recognition scale for each subject, with the scores ranging from zero (eight "no" responses, or negative recognitions, for a particular type of statement) to eight (eight positive recognitions for a particular type of statement). As Table 5.3 shows, there were in fact two scores for both stated and unstated prototypical Consequences; hence, for the relevant analyses, these two scores were averaged. To facilitate the interpretation, the raw scores were transformed into percentages of positive recognitions for each type of statements. These percentages were the input for the subsequent analyses.

In addition to percentage positive recognitions, the mean reaction times of each subject for every statement type were computed. As there was essentially no relationship between the length of the different statements and the concomitant reaction times (a Pearson correlation coefficient of -.10 was found between length of the test statement and average response latency), it was not necessary to correct the reaction times for length of the statements. In all, 10 average reaction times were computed, one for each statement type (cf. Table 5.3).

The percentage of positive recognitions and the reaction times were analyzed in a $3 \times 2 \times 2 \times 2$ ANOVA, with expertise level as between subjects factor and typicality, textual presence, and illness script component as within subjects factors. In these analyses, the inconsistent statements did not participate, because there were no stated variants of these items (see Table 5.3); thus, only two levels of the typicality factor were included. The inconsistent items were analyzed separately in a $3 \times 3 \times 2$ ANOVA, with expertise level as between subjects factor and typicality (three lewels this time: prototypical, atypical and inconsistent) and illness script component as within subjects factors. In this analysis, only the unstated items were included. Finally, percentages positive recognitions (false alarms) and reaction times for paraphrases and omissions were analyzed in a 3.2 ANOVA with expertise level as between subjects factor and paraphrases/omissions as within subjects factor.

As the reaction times for a particular item type may include both positive recognitions and failures to recognize an item, they may reflect quite different underlying processes. Consequently, some additional analyses were performed, which focused on the relationship between reaction time and quality of response: For prototypical and atypical items, reaction times for hits, false alarms, misses and correct rejections were compared, in order to investigate whether they were consistent with the findings of Yekowich and Walker (1986). The reaction time data of four of these item-response combinations (prototypical and atypical hits, prototypical correct rejections and false alarms) were analyzed in a $3 \times 4 \times 2$ ANOVA, with expertise level as between subjects factor and response type and illness script component as within subjects factors. In order to perform this analysis, the data of 10 subjects (one experienced physician, three sixth-year students and seven fourth-year students) could not be included, because these subjects did 
not produce any false alarm responses for either prototypical Enabling Conditions or prototypical Consequences, or both.

Before any of the above-mentioned analyses were performed, it was checked whether the distribution of the input data satisfied the requirements for an anal. ysis of variance. Though strictly speaking not all of our measures satisfied the criteria (e.g., hit rates for prototypical items or false alarm rates for inconsistent items showed skewed distributions), normalization procedures, like transformation of the data into logarithmic values, yjelded essentially the same results as ANOVAs of the original data. Furthermore, we preferred to use analyses of variance because the various nonparametric techniques are not equipped to deal with the complex interactions we were interested in.

It may be argued that application of the signal detection theory, which is often considered an appropriate tool to analyze recognition memory scores (cf. Parks, 1966) might yield more interesting results for the present data. However, a problem here is that we have, for most types of test statements, only eight observations per subject. This implies that in many cases, hit rates and/or correct rejection rates equalled one, or misses and/or false alarm rates equalled zero. As d" values can not be computed for these cases, it would be inevitable to exclude them from the analysis, which would considerably detract from the power of the study. In a similar vein, the memory discrimination scores employed by Smith and Graesser (1981), which are highly correlated with the d'values from the signal detection theory, yield a denominator of zero, and hence no vallue, if $p(h i t)=1$. Thus, we opted for analysis of the raw data in an ANOVA.

\section{RESULTS}

Due to an unknown equipment failure, the data for one case were not registered for two subjects. Furthermore, occasionally a reaction time had to be dism carded because an interruption or disturbance occurred during the test phase. Reaction times that exceeded 33,000 msecs were also omitted from the analysis, to avoid spuriously high averages due to one exceptional value. In all, 31 reaction times, i.e., less than .5\%, had to be excluded from the analysis.

\section{Recognition memory scores}

\section{EFECTS OF EXPERTISE LEVEL, TYPICALITY AND TEXTUAL PRESENCE ON RECOGNTTION MEMORY SCORES}

Analysis of variance of the percentage positive recognitions showed a significant main effect of typicality, $F(1,62)=89.901, p<.0001, M S_{e}=132.346$, a significant main effect of textual presence, $F(1,62)=967.919, p<.0001, M S_{e}=550.317$, a significant main effect of script component, $F(1,62)=13.162, p<.001, M S_{\mathrm{e}}=$ 168.912, a significant two-way interaction between textual presence and expertise level, $F(2,62)=4.338, p<.05, M S_{e}=550.317$, a significant two-way interaction between typicality and textual presence, $F(1,62)=27.461, p<.0001, M S_{\mathrm{e}}=119.016$, a significant two-way interaction between typicality and script component, $F(1,62)$ $=47.850, p<.0001, M S_{e}=92.513$, a significant three-way interaction between typicality, textual presence, and expertise level, $F(2,62)=3.815, p<.05, M S_{\mathrm{e}}=119.016$, and a significant threeway interaction between typicality, textual presence and 
illness script component, $F(1,62)=13.654, p<.001, M S_{e}=105.305$. No other main effect or interaction reached significance.

Table 5.4 .

Percentage positive "recognitions" as a function of textual presence and expertise level.

Textual presence

\begin{tabular}{rccc} 
Level of expertise & Stated & Unstated & Means \\
\hline Fourth-year students & 85.77 & 16.93 & 51.35 \\
Sixth-year students & 82.81 & 15.00 & 48.91 \\
Family physicians & 78.13 & 22.42 & 50.27 \\
\hline Means & 82.15 & 18.28 & 50.22 \\
\hline
\end{tabular}

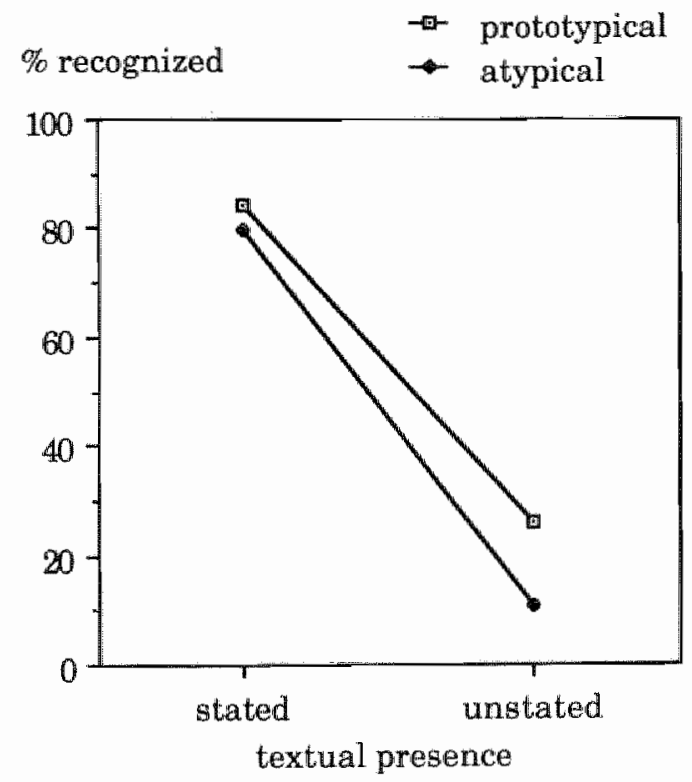

Figure 5.1

Percentage of items positively recognized as a function of typicality and textual presence. (all subjects included, $\mathrm{N}=65$ ).

Though some of the effects are rather straightforward and not very informative, they will be briefly discussed. Of the prototypical items, $55.0 \%$ were recog- 
nized, against $45.4 \%$ of the atypical items. Enabling Conditions were slightly more often recognized than Consequences, in $52.3 \%$ and $48.1 \%$ of eases, respectively. Of course, all these four percentages should equal 50\%. Stated items were correctly recognized in $82.2 \%$ of instances, while unstated items received incorrect "yes"-responses in $18.3 \%$ of instances . As can be read from Table 5.4, the less experienced subjects were somewhat more accurate than the experienced physicians; hence, the two-way interaction between textual presence and expertise level.

More interesting is the significant interaction between typicality and textual presence; this interaction, depicted in Figure 5.1, is diagnostic of memory discrimination. On the average, $84.4 \%$ of the prototypical, and $79.9 \%$ of the atypical stated items were positively recognized by the subjects, respectively. The difference between these two figures is small, compared to the difference between the prototypical and atypical unstated items: $25.7 \%$ of unstated prototypical items were positively recognized, while subjects responded "yes" to only $10.9 \%$ of the unstated atypical items. These data unambiguously support the hypothesis that memory discrimination for prototypical items is inferior to that for atypical items; as such, they are in line with other script-related research.

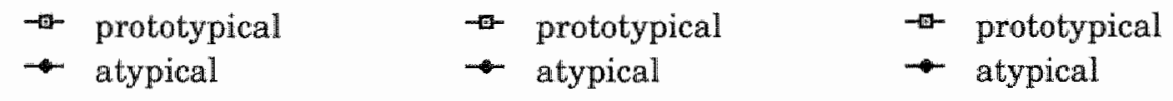

$\%$ recognized

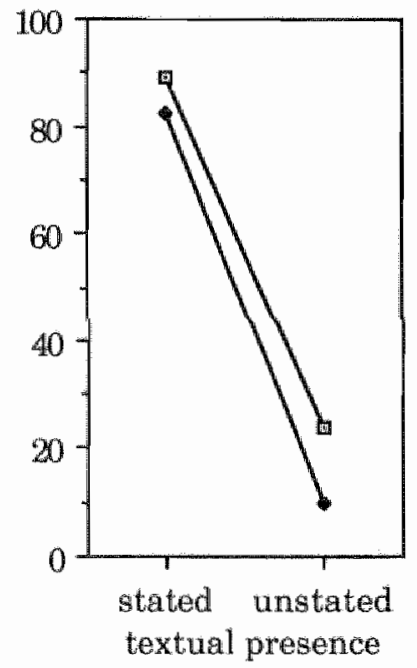

Fourth-year students
$\%$ recognized

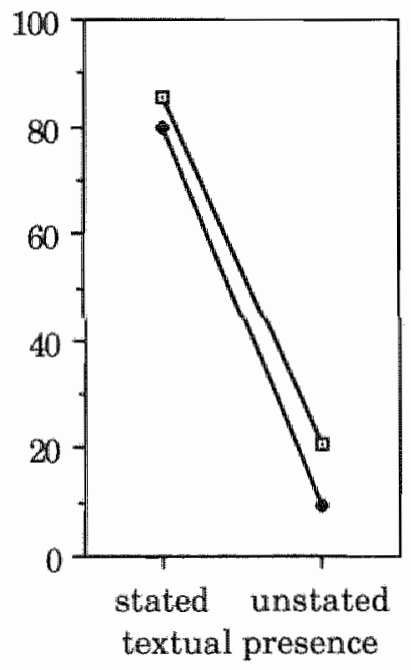

Sixth-year students
To recognized

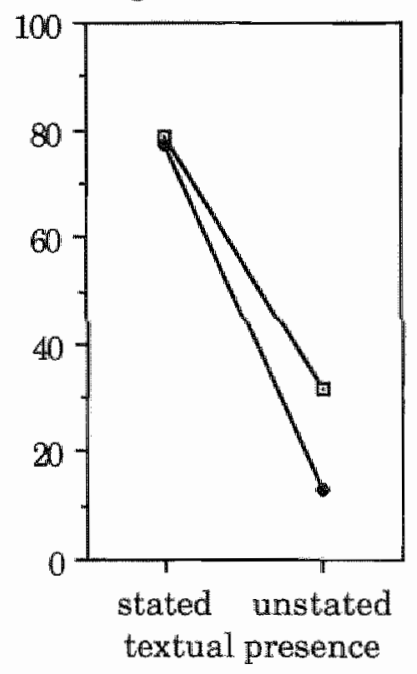

Family physicians

Figure 5.2

Hit rates and false alarm rates (expressed as percentage positive recognitions) as a function of expertise level, typicality, and textual presence. 
Even more pertinent to the present theory, however, is the finding of a significant three-way interaction between typicality, textual presence, and expertise level. Figure 5.2. shows this interaction. It implies that the contribution of the data of subjects at different levels of expertise to the two-way interaction displayed in Figure 5.1 was not equal. Comparison of the three panels of Figure 5.2 suggests that this two-way interaction between typicality and textual presence can be accounted for mainly by the data of the experienced physicians; their performance on the stated items was about equal for prototypical and atypical statements, while they had more difficulty in correctly rejecting unstated prototypical than unstated atypical items. For fourth-year and sixth-year students, the interaction effect apparently is of lesser importance, or may even be completely absent.

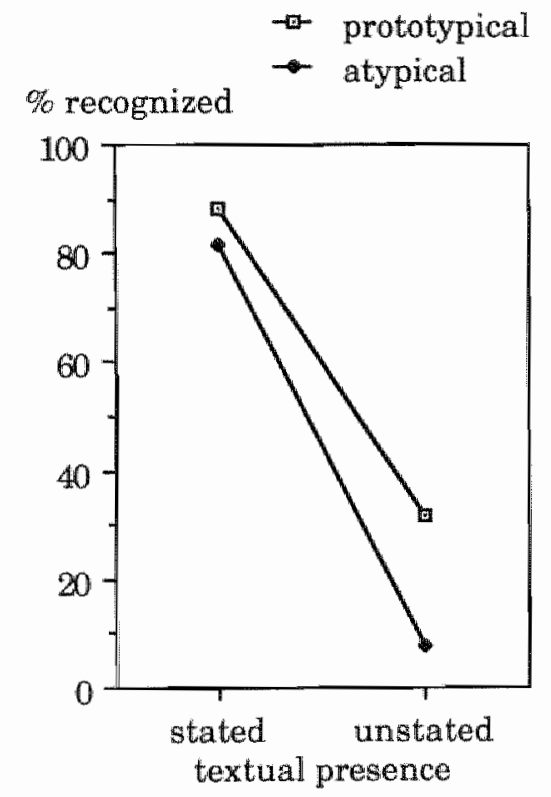

Enabling Conditions
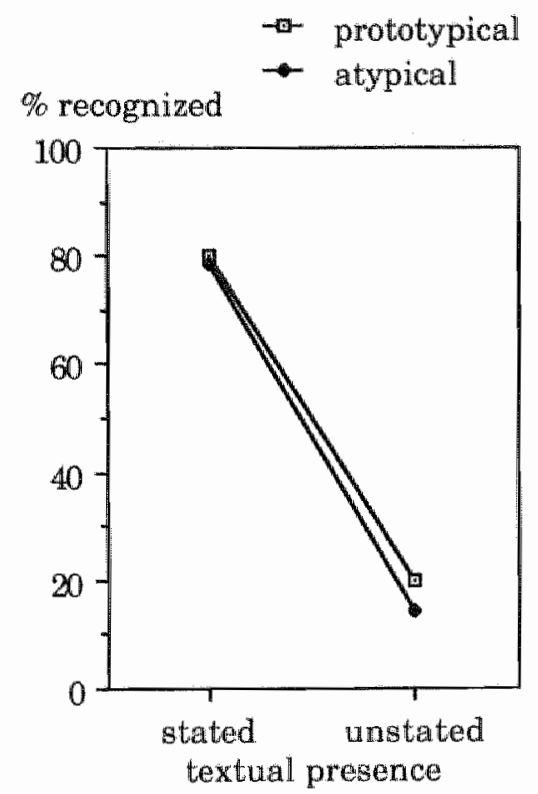

Consequences

Figure 5.3

Interaction between typicality and textual presence on percentage of positive recognitions for Enabling Conditions (left hand panel) and Consequences (right hand panel).

From Figure 5.2, it might also be read that even the experienced family physicians do not show the expected (cf. Graesser et al., 1979) zero memory discrimination for prototypical statements: Their false alarm rates for this type of statements was not nearly as high as their hit rates (about $32 \%$ false alarms against $79 \%$ hits). On the other hand, the false-alarm rates for atypical items, in the present study about $13 \%$ for the experienced family physicians and about $10 \%$ for the 
less experienced subjects, seem to be largely comparable to the proportion of .16 reported by Graesser et al. (1979).

As far as the two illness script components, i.e., Enabling Conditions and Consequences, are concerned, the two-way interaction between typicality and illness script component, and the three-way interaction between typicality, textual presence, and illness script component are a consequence of relatively poor mernory discrimination for Enabling Conditions, compared to memory discrimination for Consequences. Figure 5.3 shows the interaction between typicality and textual presence, for Enabling Conditions (left hand panel), and for Consequences (right hand panel). From this figure, it can be read that the general typicality by textual presence interaction depicted in Figure 5.1 should be attributed to the data on Enabling Conditions, rather than to those on Consequences.

As the expected interactions between expertise level and illness script component, including the four-way interaction between expertise level, typicality, textual presence, and illness script category, did not reach significance, the role of Enabling Conditions seems to be similar at all three levels of expertise. Thus, no evidence was found for the hypothesized differential role of Enabling Conditions for experts and non-experts.

In summary, the results of the analysis of the percentage of positive recognitions favors the hypothesis that atypical statements show generally better memory discrimination than prototypical statements, a finding that is in line with previous script related research. Furthermore, there is evidence that the differential effect of typicality is more pronounced for experienced subjects than for advanced students, a finding that can be interpreted in support of the prediction that experienced subjects show more "script-like" behavior on the type of recognition task employed in this study. However, the expectation that this expertise effect could be attributed to the data on Enabling Conditions, rather than on those of Consequences, was not borne out.

\section{THE EFFECTS OF EXPERTISE LEVEL AND ILLNESS SCRIPT COMPONENT ON FALSE ALARM SCORES FOR. INCONSISTIENT INFORMATION}

Over all subjects and both illness script components, slightly more than one out of every twenty inconsistent items was falsely recognized; thus, the number of false alarms was very limited. A $3 \times 3 \times 2$ analysis of variance of false recognition scores, with expertise level as between subjects factor and typicality (three levels: prototypical, atypical, and inconsistent) and illness script component as within subjects factor, revealed only a significant main effect of typicality, $F(2,124)=$ $119.311, p<.0001, M S_{\mathrm{e}}=119.514$, and a significant interaction between typicality and illness script component, $F(2,124)=34.813, p<.0001, M S_{e}=82.876$; neither the main effects of expertise level and illness script component, nor any of the other interactions were significant.

The interaction between typicality and illness script component will not be further discussed, because it can be completely accounted for by the data on prototypical and atypical items, and hence has already been described in the previous section. In fact, the false alarm rates for inconsistent Enabling Conditions and inconsistent Consequences were highly similar: $4.6 \%$ and $5.6 \%$, respectively. Overall, prototypical, atypical, and inconsistent unstated items were falsely recognized in $25.7 \%, 10.9 \%$, and $5.1 \%$, of instances, respectively. As these latter two values are relatively close to zero, an analysis of variance might not yield reli- 
able results; thus, a Wilcoxon Signed Rank Test was performed to compare the false recognition scores for atypical and inconsistent statements. This test yielded a $Z$-value (corrected for ties) of $-4.309, p_{\text {(one-tailed) }}<.0001$. Therefore, it may safely be concluded that atypical items were falsely recognized more often than inconsis. tent items. This conclusion has mainly methodological implications; it supports the assumption that atypical information has a significantly higher base-line probability, given the activated illness script, than inconsistent information.

\section{THE EFFECT OF EXPERTISE LEVEL ON RECOGNITION SCORES FOR PARAPHRAS- ES AND OMISSIONS}

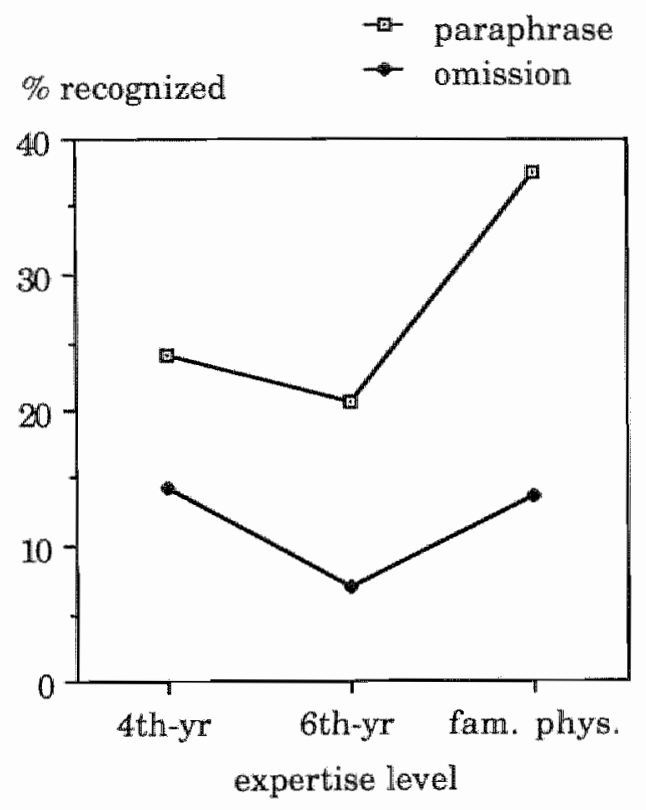

Figure 5.4

Percentage of false recognitions as a function of expertise level and type of unstated prototypical Consequences.
Finally, recognition data for the two types of unstated prototypical Consequences, i.e., the paraphrases and the omissions, were compared. Figure 5.4 shows the results. A $3 \times 2$ analysis of variance with expertise level as between subjects factor and type of item (paraphrase or omission) as within subjects factor revealed a marginally significant effect of expertise level, $F(2,62)=2.538, p<$ .1, $M S_{\mathrm{e}}=592.213$, a significant effect of item type, $F(1,62)=43.961, p$ $<.0001, M S_{\mathrm{e}}=183.329$, and a significant interaction between expertise level and item type, $F(1,62)$ $=3.308, p<.05, M S_{\mathrm{e}}=183.329$ on the percentage false recognitions of prototypical unstated Consequences. Generally, the sixth-year students were the most accurate subjects, with $13.8 \%$ false recognitions, while the experienced physicians mistakenly responded "yes" to these items in $25.5 \%$ of cases. The fourth-year students fell somewhere in-between, with $19 \%$ false alarms.

More interesting, however, is

the finding of a significant effect of item type. Overall, almost $28 \%$ of paraphrases were falsely recognized, against somewhat less than $12 \%$ of omissions. This is an important finding. It reveals that omissions are not more often falsely recognized than atypical unstated items, which elicited "yes" responses in about $11 \%$ of instances. From these data, it can be concluded that subjects, regardless of their expertise level, are not inclined to infer unstated prototypical complaints, signs, and symptoms. The exact wording, and probably other surface features of the actually presented information, however, are to a large extent lost, as the relatively high proportion of false recognitions of paraphrases indicates; moreover, the finding of a significant interaction between expertise level and type 
of unstated prototypical Consequence shows that experienced physicians are more strongly inclined than subjects at lower levels of expertise to encode case information by meaning and to "forget" the surface features of the presented information. However, even for paraphrases, the data of the experienced family physicians are quite remote from zero memory discrimination: They scored $73.4 \%$ hits for prototypical stated Consequences against $37.5 \%$ false alarms for paraphrases, $t(22)=7.399$, p(one-tailed) $<.0001$. Thus, though subjects at all levels of expertise do encode and remember information about the literal wording of case information, experienced physicians are more inclined to forget this information and to use meaning to respond to paraphrased information.

\section{Recognition reaction times}

\section{EFFECT OF EXPERTISE LEVEL, TYPICALITY, AND TEXTUAL PRESENCE ON TECOGNITION REACTION TTMES}

Analysis of variance of the reaction times for the experimental test statements revealed a significant main effect of expertise level, $F(2,62)=4.059, p<.05$, $M S_{\mathrm{e}}=4,554,178.417$, a significant main effect of typicality, $F(1,62)=11.056, p<$ $.005, M S_{e}=153,739.913$, a significant two-way interaction between typicality and textual presence, $F(1,62)=22.731, p<.0001, M S_{e}=213,206.207$, a significant twoway interaction between textual presence and illness script component, $F(1,62)=$ $5.547, p<.05, M S_{\mathrm{e}}=341,682.920$, and a significant three-way interaction between typicality, textual presence, and illness script component, $F(1,62)=11.412, p<$ $.005, M S_{e}=293814.845$. None of the other main effects or interactions were significant, though two of them, i.c., the two-way interaction between typicality and illness script component, $F(1,62)=2.872, p<.1, M S e=386,731.176$, and the threeway interaction between expertise level, typicality, and script component, $F(2,62)$ $=3.083, p<.1, M S e=386,731.176$, just failed to reach it. As the main factor of textual presence does not participate in these latter interactions, they are probably of limited relevance and difficult to interpret; hence, they will not be further discussed.

Generally, it took fourth-year students, sixth-year students and family physicians 3103,2692 and $2467 \mathrm{msec}$, respectively, to react to the test items. As such, these results corroborate those of the study described in the previous chapter, in which also a positive relationship between expertise level and processing speed was found. In addition, prototypical items took significantly more time to be responded to than atypical items (2836 msec versus 2668 msec, respectively). According to Nakamura and Graesser (1985) and Yekovich and Walker (1986), this typicality effect should be accounted for especially by long reaction times for correct rejections of prototypical, unstated test items; we will return to this issue later.

The interaction between typicality and textual presence is depicted in Figure 5.5. From this figure, it can be read that prototypical and atypical stated items are recognized about equally fast, while prototypical unstated items take about $14 \%$ more time than atypical unstated items. Though this latter difference is not as impressive as the one reported by Yekovich and Walker (1986), it is in fact consistent with the hypothesis.

On the other hand, the finding that prototypical and atypical stated items show response latencies of about equal size, is not in line with the predictions: It was expected that atypical stated items would show smaller reaction times than 
prototypical stated items. Probably, the present experimental design facilitated positive recognition of both types of presented items, for example on basis of surface features - after all, the retention interval was quite short - while item meaning plays a more important role if no immediate recognition occurs, a situation in which subjects are able to reject atypical items more quickly because their meaning does not fit that well in the script content.

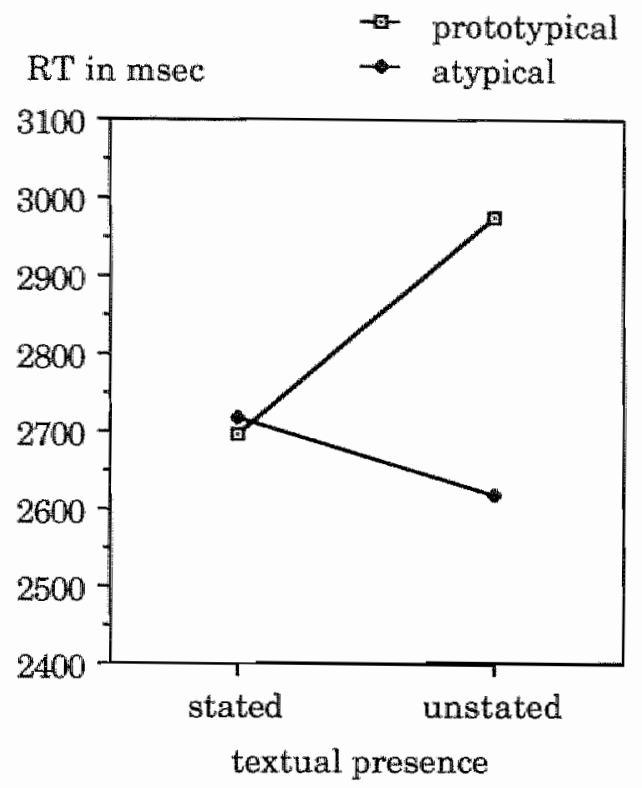

Figure 5.5

Effects of typicality and textual presence on reaction times (expressed in msec).

panel) and Consequences (right hand panel).

Like in the case of the recognition scores depicted in Figure 5.3 , the typicality by textual presence interaction effect for the reaction times can be accounted for largely by the data on the Enabling Conditions statements; for Consequences, the difference in recognition speed between prototypical and atypical unstated items is relatively small ( 2801 versus $2913 \mathrm{msec}$, respectively), whereas for Enabling Conditions, this difference is much larger (2433 versus $3037 \mathrm{msec}$, respectively). Thus, as far as the reaction time data are concerned, Enabling Conditions also seem to behave more script-like than Consequences, with slow reaction times for prototypical unstated items and fast reaction times for atypical unstated items.

Illness script component also featured in a difficult to interpret two-way interaction with textual presence. This interaction implies that response latencies for stated and unstated Enabling Conditions are of about equal length (2769 msec and $2735 \mathrm{msec}$, respectively), while subjects responded faster to stated Consequences than to unstated Consequences (2646 $\mathrm{msec}$ versus $2857 \mathrm{msec}$, respec- 
tively). The relevance of this interaction in light of the present theory is difficult to assess, because the interaction covers both prototypical and atypical statements; in addition, the size of the effect is not impressive. A possible explanation could be that Consequences, unlike Enabling Conditions, were sometimes phrased in typical medical jargon, which may be easier to recognize than the more "plain" expressions employed to describe patient characteristics.

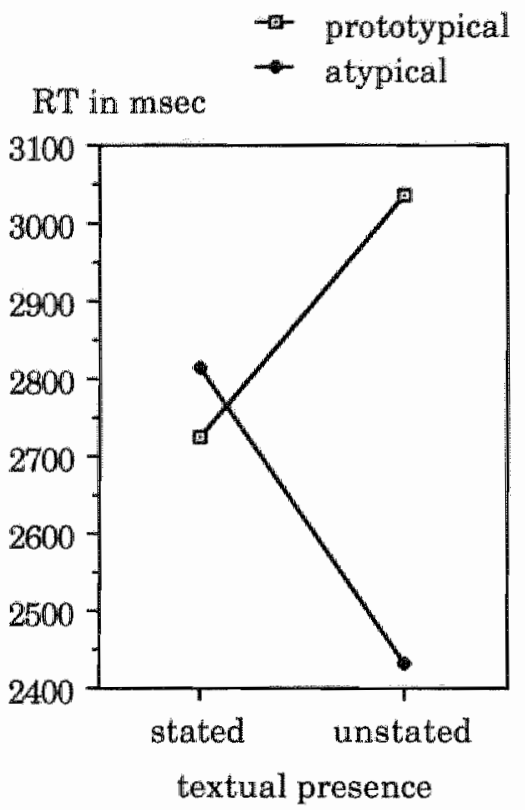

Enabling Conditions

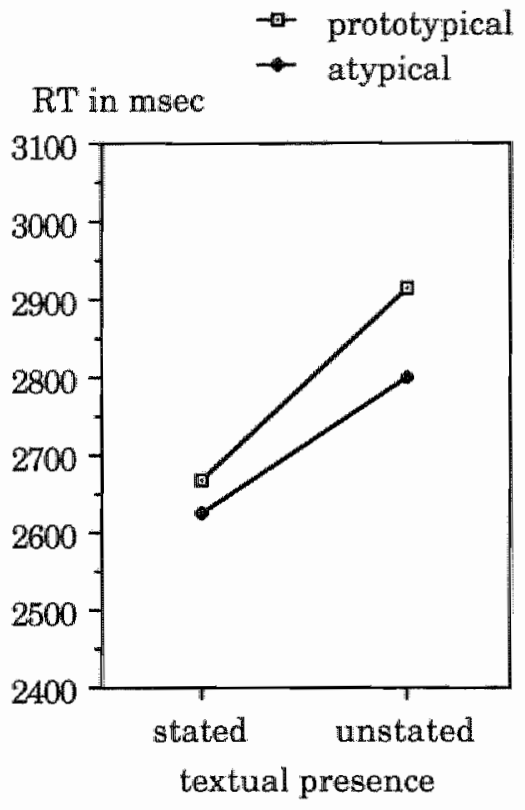

Consequences

Figure 5.6

Effects of typicality and textual presence on reaction times (expressed in msec), for Enabling Conditions (left hand panel) and Consequences (right hand panel)

\section{THE EFFECTS OF EXPERTISE LEVEL AND ILLNESS SCRIPT COMPONENT ON REACTION TTMES FOR INCONSISTENT INFORMATION}

According to the script theory, subjects should be able to reject inconsistent information (i.e., unstated information that is implicitly or explicitly contradicted by one of the statements in the case text), accurately and quickly. The first part of this hypothesis indeed was confirmed by the data on the proportion positive recognitions. In this section, the results of the analysis of the reaction times will be presented. Like the recognition scores, the reaction times for inconsistent items will be compared with those for prototypical and atypical unstated items. A $3 \times 3 \times 2$ ANOVA with expertise level as between subjects factor and typicality (three levels: prototypical, atypical, and inconsistent) and illness script component as within subjects factor, revealed the expected main effect of expertise level, $F(2,62)=$ 
$4.623, p<.05, M S_{\mathrm{e}}=2,783,191.225$, but also a significant main effect of typicality, $F(2,124)=22.413, p<.0001, M S_{\mathrm{e}}=345,350.650$, and a significant main effect of illness script component, $F(1,62)=28.862, p<.0001, M S_{e}=186,683.057$. In addition, a significant interaction between typicality and illness script component was found, $F(2,124)=10.900, p<.0001, M S_{e}=314,163.846$. All three interactions with expertise level included, however, failed to reach significance. The most important data are summarized in Table 5.5.

Table 5.5

Reaction times (expressed in msec) as a function of typicality and illness script component.

Typicality Enabling. Conds. Consequences Means

$\begin{array}{llll}\text { Prototypical } & 3037 & 2913 & 2975\end{array}$

$\begin{array}{llll}\text { Atypical } & 2433 & 2801 & 2617\end{array}$

$\begin{array}{llll}\text { Inconsistent } & 2279 & 2744 & 2512\end{array}$

$\begin{array}{llll}\text { Means } & 2583 & 2820 & 2701\end{array}$

Subjects at higher levels of expertise were generally faster than subjects at lower levels of expertise; reaction times of fourth-year students, sixth-year students and family physicians were 3044,2628 , and 2438 msec, respectively. This is completely in keeping with the expectations. However, the absence of any interaction between expertise level and typicality or illness script component indicates that the pattern of reaction times is similar for these variables at the three levels of expertise included.

From Table 5.5, it can be read that, consistent with the expectations, items that deviated to a large extent from prototypical information showed shorter response latencies. Furthermore, Enabling Conditions are in general easier to respond to than Consequences, but this effect can be accounted for completely by the atypical and inconsistent Enabling Conditions: Apparently, this type of items can be quickly rejected (there were few false alarms for atypical and inconsistent items), a conclusion that already followed from the previous reaction time analysis, in which the inconsistent items were not yet included. A remarkable finding is that it takes subjects relatively long to respond to inconsistent Consequences, even though these items explicitly contradict a complaint, sign or symptom described in the case. A possible explanation of this phenomenon could be that inconsistent Enabling Conditions probably are contradictory to the general picture the subject has formed of the patient described in the case (e.g., an older male patient), while inconsistent Consequences contradict more specific items.

It may be objected that it is not appropriate to compare reaction times for inconsistent items, which are based almost exclusively on correct rejections, with reaction times for prototypical unstated items, these latter being of a much more hybrid nature, i.e., based on both correct rejections and false alarms. However, a repeated analysis of the data based on solely the reaction times for the correct rejection responses of each type, yielded essentially the same results, except that the expertise main effect is no longer significant. In summary, as far as reaction 


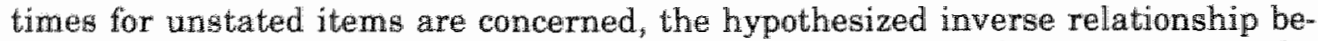
tween item typicality and reaction time was indeed found; but contrary to the expectations, Enabling Conditions seem to lead to more script-consistent behavior than Consequences, for inexperienced as well as experienced subjects.

\section{THE EFFECT OF EXPERTISE LEVEL ON REACTION TIMES FOR PARAPHRASES AND OMISSIONS}

Does it take more time to react to a paraphrase or an omission? As a consequence of theoretical considerations, it was hypothesized that experienced physicians would have about equal difficulty in rejecting paraphrases and omissions, while fourth-year students would relatively quickly recognize that omissions were never presented - because omissions would not be activated in inexperienced subjects, while they would in experienced subjects, as a consequence of illness script activation. The actual reaction time data are displayed in Figure 5.7.

A $3 \times 2$ analysis of variance with expertise level as between subjects factor and paraphrase/ omission as within subjects factor showed a significant main effect of expertise level, $F(2,62)=3.265$, $p<.05, M S_{\mathrm{e}}=1,347,606.170$, a significant main effect of type of statement, $F(1,62)=10.902, p<$ $.005, M S_{\mathrm{e}}=737,823.296$, while the interaction between expertise level and type of statement just missed significance, $F(2,62)=2.692, p<$ $.1, M S_{\mathrm{e}}=274,052.354$. The effect of expertise level was as expected, though the difference between sixth-year students' and experienced physicians' reaction times was negligible (reaction times were 3276,2737 and $2718 \mathrm{msec}$ for fourth-year students, sixth-year students and family physicians, respectively). Figure 5.7 also shows that paraphrases were responded to more slowly than omissions. Though the interaction between expertise level and type of statement just failed to reach significance, there is probably a tendency for the difference in reaction speed between paraphrases and omissions to increase with expertise level, where a decrease would be more in line with expectations. Together with the considerably higher rate of false alarms for paraphrases than for omissions (cf. Figure 5.4), it may be concluded that experienced subjects are especially error prone when faced with paraphrases, i.e., more than less experienced subjects 
they are inclined to code case information by meaning rather than by surface features.

However, unstated information, even if it is completely prototypical given a particular disease, is not implicitly inferred by subjects at any expertise level, experienced physicians included. Thus, though subjects represent the information in a case probably at a higher level than the exact text base, no support was found for the hypothesis that information prototypical with respect to an activated illness script is implicitly activated upon hearing the header of the script: An illness script is not instantiated by default in that fashion. Even for prototypical patients, experienced physicians are not inclined to add complaints or symptoms that are not explicitly presented.

\section{Reaction times for different types of recognition responses: Comparison of the present results with the Yekovich and Walker (1986) data}

To a certain extent, average reaction times for a particular type of statement do not provide a proper impression, because these reaction times may be associated with two different recognition responses, and hence with two different underlying responses. Generally, four types of recognition responses can be distinguished, according to the specific combination of stimulus and response: hits, false alarms, correct rejections, and misses. Consequently, it may be interesting to analyze reaction times separately for these different response qualities. According to Yekovich and Walker (1986), four combinations of responses and reaction times are especially interesting: Reaction times for correct positive recognitions of (prototypical and atypical) stated items, reaction times for correct rejections of prototypical unstated information, and reaction times for false alarm responses to prototypical unstated items. A $3 \times 4 \times 2$ analysis of variance $(N=55$; ten subjects that did not produce any false alarms for prototypical items had to be excluded) of reaction times with expertise level as between subjects factor and response type (hits, correct rejections, and false alarms for prototypical items, and atypical hits) and illness script component as within subjects factors, revealed a significant main effect of response type, $F(3,156)=21.034, p<.0001, M S_{e}=1,037,603.620$, a significant main effect of illness script component, $F(1,52)=5.526, p<.05, M S_{\mathrm{e}}=$ $755,153.773$, and a significant three-way interaction between expertise level, response type, and illness script component, $F(6,156)=5.780, p<.0001, M S_{\mathrm{e}}=$ $677,428.331$. The main effect of expertise level and the three two-way interactiong were not significant.

In Table 5.6, the average reaction times associated to the four types of responses are displayed, and compared to those reported by Yekovich and Walker (1986). As the reaction times in our study were in general substantially longer than those reported by Yekovich and Walker (1986), our task was apparently more difficult. Moreover, whereas the Yekovich and Walker (1986) subjects were particularly slow to correctly reject prototypical unmentioned itens, in our study especially false alarm responses were associated with slow reactions. Though the present results also suggest a three stage model of responding to the test items, the nature of this model differs from that proposed by Yekovich and Walker (1986). Our data indicate that presented items, both prototypical and atypical, are relatively quickly positively recognized; if this recognition process fails, some of the unstated prototypical items can be rejected quite definitely, probably as a consequence of negative recognition of certain surface features (e.g.: "no, the tension was not presented quantitatively"), while if surface features provide no cue, or in.- 
formation about surface features in memory has decayed, meaning is taken into consideration, with a corresponding risk of false alarms. Thus, whereas in the Yekovich and Walker (1986) study, false alarms are considered the consequence of a quick, but spurious, meaning-based recognition response to prototypical items, in our study, false alarms seem to be more the outcome of a slow, error prone, meaning-based decision process, contingent upon failure of both positive and negative recognition. In both studies, it is not unlikely that the pattern of reaction times reflects an underlying process of script-based activation. The finding that it took our subjects longer to correctly reject prototypical items than atypical items (2864 msec ${ }^{18}$ versus $2572 \mathrm{msec}$, respectively, $F(1,62)=12.483, p<.001$, $M S_{\mathrm{e}}=214,884.742$, bolsters the conclusion that in the second stage of the recognition process, meaning based processing interferes to a certain extent with more immediate negative recognition.

\section{Table 5.6}

Average reaction times (expressed in msec) associated to four types of test itemresponse combinations $(\mathrm{N}=55)$.

Type of response Present study

Hit, prototypical item

Hit, atypical item

Correct rejection, prototypical item

False alarm, prototypical item
2609

2513

2901

3478
Yekovich \& Walker

(1986)

1093

889

\begin{tabular}{ccc} 
False alarm, prototypical item $\quad 3478 \quad 1124$ \\
\hline
\end{tabular}

Analysis of the main effect of illness script component showed that reaction times associated with Enabling Conditions were generally slower than those associated with Consequences (2963 msec versus $2787 \mathrm{msec}$ ); however, the relevance of this finding is probably limited, because such different types of responses are involved. The significant three-way interaction between expertise level, response type and illness seript component is depicted in Figure 5.8, separately for each expertise level; this interaction suggests that for the three levels of expertise, the pattern of reaction times for Enabling Conditions and Consequences over the four response types, is not identical.

The most striking feature of the data depicted in Figure 5.8 is the substantial decrease, with expertise level, in reaction times of false alarms to prototypical Enabling Conditions: it takes fourth-year students almost $2000 \mathrm{msec}$ longer to emit a false alarm to this type of items than experienced physicians. This finding suggests that, if false alarm responses to prototypical items are partly meaning based, this meaning factor may exert a stronger influence in experienced physicians than in students. In other words: meaning, at least for Enabling Conditions, may be stronger activated in experienced subjects, which becomes manifest in relatively fast false alarm reaction times (i.e., the influence of meaning quickly

18 This value differs from the corresponding entry in Table 5.6 since more subjects were included in the present analysis. 
"overrides" the influence of not being able to find a tag). This finding is buttressed by the data on the percentages of false recognitions of unstated prototypical Enabling Conditions: this type of items is "recognized" by $38.0 \%, 27.5 \%$ and $28.6 \%$ of family physicians, sixth-year students, and fourth-year students, respectively ${ }^{19}$.

$\mathrm{RT}$ in msec
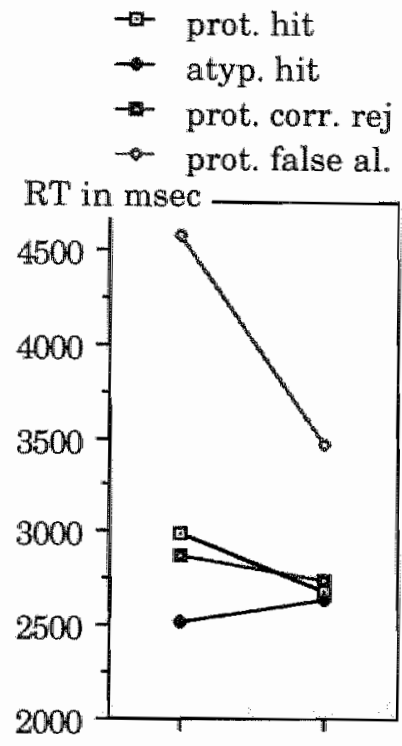

ECs Cons

illness scr. comp.

Fourth-year students $\rightarrow$ prot. hit

$\rightarrow$ atyp. hit

$\rightarrow$ prot. corr. rej

prot. false al.

$\mathrm{RT}$ in msec

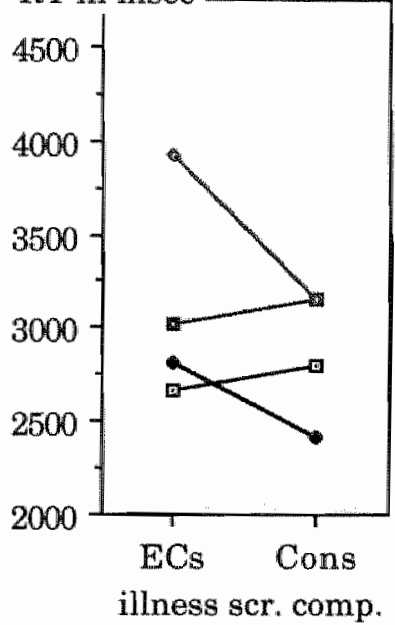

Sixth-year students
- prot. hit

$\rightarrow$ atyp. hit

$\rightarrow$ prot. corr. rej

$\rightarrow$ prot. false al.
$\mathrm{RT}$ in msec

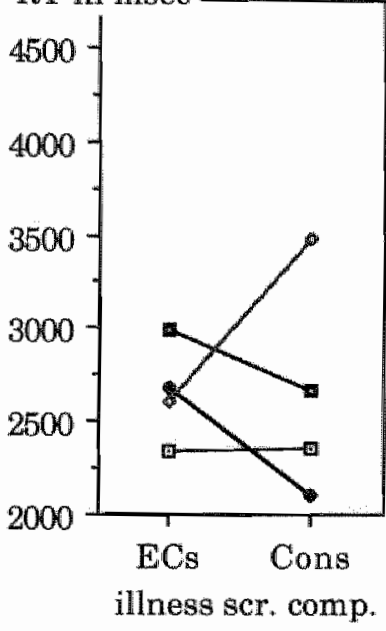

Family physicians

Figure 5.8

Reaction times (expressed in msec) as a function of response type and illness script component, for the three individual expertise levels.

Thus, unstated prototypical Enabling Conditions are relatively quickly "recognized" by experienced physicians, probably as a consequence of implicit activation, while fourth-year students (and, to a lesser extent, sixth-year students) apparently search for a memory tag, ultimately to decide that there is indeed one. The reaction times for false alarms to unstated prototypical Consequences, on the other hand, suggest that subjects at all three expertise levels employ similar recognition processes, and that these processes are, even for experts, not exclusively meaning based. It can not be excluded, on basis of these data, that searching for a memory tag is contingent upon the presence of specific surface features in the input, like words which can be immediately recognized as originating from

19 However, a oneway analysis of variance showed that this difference did not exced the significance threshold, $F(2,62)=2.236, p<.12, M S_{e}=333.776$. 
the medical domain (e.gn, words beginning with "hyper-"); if these words occur more often in Consequences than in Enabling Conditions, this could explain why even experienced physicians are not strongly inclined to show meaning-based false recognition of Consequences, and why, if they sometimes happen to do so, the recognition process results in a slow, rather than a quick, reaction (cf. Figure 5.8 , right-hand panel).

\section{DISCUSSION}

In this study, the influence of expertise level, typicality, textual presence, and illness script component on memory performance, operationalized as recognition responses and recognition reaction times, was investigated. The aim of the study was to find out whether the illness script, as a specific variant of the classical (Schank \& Abelson, 1977) script and the schema (Bartlett, 1932 /1954; Graesser \& Nakamura, 1982), is a psychologically viable concept. The general approach of the study was to test, in an experiment, the prediction that the memory performance characteristics of these classical scripts and schemata would also apply to illness scripts. Such a test was achieved by replicating the experimental design previously applied in script research. In addition, some more specific hypotheses with respect to the development of illness scripts and the presumed differential role of Enabling Conditions and Consequences in this development, were investigated.

Though the present results paralleled in a meaningful way those of earlier script related research, also a number of predictions could not be borne out, particularly as far as the developmental aspects of illness scripts are concerned. In keeping with previous work were the findings that unmentioned prototypical information had a higher chance of being falsely recognized than unmentioned atypical information, while inconsistent information led to extremely low false alarm scores. Moreover, for stated items, such a typicality effect was essentially absent.

Similarly, the reaction times also showed a significant interaction between textual presence and typicality. As expected, it took subjects relatively much time to respond to unstated prototypical information, while both atypical unstated information and inconsistent information were quickly reacted to. Thus, in contrast with the explicit instruction to the subjects to judge the test items by textual presence and not by content, meaning definitely "seeps through" in responding to unstated information. The reaction times for stated information, on the other hand, were about the same for both typicality values. These latter results indicate that both prototypical and atypical literally stated information can be recognized on basis of surface features for which a memory tag has been created: Meaning does not seem to exert a large influence on the size of the response latencies for stated information.

A remarkable and unexpected finding was that the typicality by textual presence interaction, for recognition scores as well as for reaction times, had to be attributed to the data on the Enabling Conditions, rather than to those on the Consequences (cf. Figure 5.3 and 5.6). Presently, the relevance of this finding is unclear. It was hypothesized that Consequences probably are easier to recognize on basis of surface features, while Enabling Conditions are more part of the general image a physician forms of a patient; this hypothesis can indeed explain why atypical unstated Enabling Conditions are relatively quickly and accurately rejected, while prototypical unstated Enabling Conditions show slow reaction times 
and a large percentage of false alarms. However, this hypothesis would also predict that memory performances for stated Consequences would be generally superior to those for stated Enabling Conditions, because the memory tags formed for stated Consequences should be easier to retrieve than those for stated Enabling Conditions; this, in fact, was not the case. Nevertheless, the data do indicate that Enabling Conditions and Consequences play a differential role in the memory representation of illness scripts.

A more fine-grained analysis of the reaction time data revealed that the long reaction times for prototypical, unstated items could to a large extent be attributed to the response latencies for false alarms, rather than to those for correct rejections. Though this finding is not consistent with the results of Yekovich and Walker (1986), who report longer reaction times for correct rejections than for false alarms, this lack of correspondence can probably be attributed to differences in task instructions: As our subjects were instructed to decide whether a specific Information item had been literally presented in the case, it may be no surprise that the decision to correctly reject an item that was not positively recognized (i.e., to decide that no memory tag is present) was made faster than the decision that an item had been literally presented, when this in fact had not been the case (i.e., a false alarm). Subjects in the Yekovich and Walker (1986) study had to decide whether an information item had been presented or not, regardless of the exact way it was expressed. In their model, false alarms are quick responses to the content of a test item, and correct rejections are slow responses following the inability to find a memory tag, while in our study correct rejections can be viewed as fast responses to the definite absence of a memory tag, and false alarms are slow responses in situations in which subjects are uncertain whether a tag had been formed. In our opinion, in the Yekovich and Walker (1986) study, the role of sur"face features of the input, for which tags may have been formed, is unclear.

Nevertheless, the results of the present study also suggest a three stage model of responding, though one that differs somewhat from the other models: In the first stage, an actually presented item is usually quickly and positively recognized as a consequence of the presence of a tag; similarly, an atypical unpresented item is quickly rejected. If a prototypical unstated item is put to test, however, two interfering processes come into play: A meaning-based process that proposes a "yes" answer (thus, leading to a false recognition), and a recognition-based process that proposes a "no" answer, (hence, resulting in a correct rejection). Though it generally takes some time to resolve this response competition, the duration of the process probably depends on the strength of the negative recognition response, (i.e., the reaction "no, this piece of text was not part of the case"); if this response is strong, the item will be correctly rejected, if this response is weak, the subject will eventually, after some additional decision time, respond to the meaning-based "yes"-tendency, and hence produce a false alarm. "Though this model is constructed post-hoc to explain the data in Table 5.6, and thus certainly can not be considered experimentally confirmed, the fact that the figures in this table suggest a stage model of recognition responding at least similar in structure to the models proposed in earlier script-based research, confirms the notion that scriptlike structures may be the source of the recognition response patterns found in the present study.

As far as the developmental aspects of the illness script theory are concerned, the present results do certainly not unequivocally provide support for the theory. The only real indication of a developmental trend in script development was the finding, for the recognition scores, that the typicality by textual presence interaction was mainly due to the data of the experienced physicians, rather than 
to those of the less expert subjects. In this respect, our family physicians show script consistent memory performances (i.e., large recognition differences between atypical and typical unstated information), while the two student groups merely showed, across the board, somewhat higher positive recognition scores for prototypical items. Furthermore, though this typicality by textual presence interaction could be accounted for by, on the one hand, the recognition performance of the experienced physicians, and, on the other hand, the recognition scores for the Enabling Conditions, the absence of a significant four-way interaction between expertise level, typicality, textual presence, and illness script component, sug. gests that these findings are additive rather than interactive. Thus, the finding that the performances of experienced physicians were more in accordance with scripted processing than those of advanced students, can not exclusively be attributed to better integration of Enabling Conditions into illness script structures: Integration of Consequences should be equally credited for this.

As far as the reaction times are concerned, the effect of expertise level was confined to a general speed advantage for the more experienced subjects; other relationships were virtually absent. Particularly the absence of an interaction between expertise level, typicality, and textual presence suggests that subjects at all three levels of expertise show script consistent behavior (i.e., slow reaction times for unstated, prototypical information). The finding that, with increasing expertise level, the reaction times for false alarms to prototypical Enabling Conditions diminish sharply, whereas those to Consequences do not change (see Figure 5.8 ), is too remote from current ideas about illness script development to enable an easy explanation.

In summary, our data do not support the hypothesis of a differential development of Enabling Conditions and Consequences; indeed, they do not even hint at the possibility. An explanation for this finding might be that the recognition task is not sufficiently sensitive to uncover expert-novice differences in knowledge of Enabling Conditions and Consequences, because subjects are required to recognize Enabling Conditions against the background of a diagnosis, rather than the other way round. The differences between Enabling Conditions and Consequences we actually did find in this study (cf. Figures 5.3 and 5.6), may be a consequence of the different role of both illness script components in the present task, i.e., Enabling Conditions "coloring" the complete picture of a patient (e.g., male, old, coronary disease), while Consequences involve more specific information (heavy perspiration, chest pain, shortness of breath). Subjects at all three levels of expertise are probably equally equipped to deall with both illness script components in a recognition task; hence, the absence of significant interactions between expertise level and illness script component.

The differences in recognition memory for the two types of unstated prototypical items, I.c., the paraphrases and the omissions, show that, though subjects encode presented information by meaning, and hence are inclined to forget the exact wording (i.e., the tags initially formed for surface features tend to decay rapidly), they do not automatically infer prototypical unstated disease information: The false recognition scores for the omissions were comparable to those for atypical unstated items, both falling in the range of $10.15 \%$. This finding seems inconsistent with the earlier script related research, in which often much higher false recognition scores for unstated information, deliberately deleted from the scripted text, are reported; indeed, it is also hard to reconcile with the high proportion of false intrusions reported in previous research on memory for medical information (e.g., Arkes \& Harkness, 1980). If physicians are inclined to falsely recall disease consistent, but unstated, findings, signs and symptoms, why should they not 
be equally inclined to falsely recognize this kind of information? Some remarks, pertinent to this issue, can be phrased. First, the retention interwal in our study was relatively short (between the presentation of a case during the study phase and the corresponding test items, generally $20-25$ minutes elapsed). Previous work (e.g., Smith \& Graesser, 1981) has shown that, with increasing retention interval, memory performance becomes more script-based; hence, it is feasible that if the test task had been administered after, for instance, one week, subjects would have had more difficulty in correctly rejecting the omissions. Second the Consequences in an illness script may have a different function, compared to the actions in a regular script. This difference in function may be considered a consequence of the fact that regular scripts are part of a causal goal structure, while illness scripts are not. Indeed, Graesser and Nakamura (1982) assigned a "necessity score" to quantify the degree of compulsiveness such inferences are made with. For example, returning to the "Jack story" presented in Table 5.1, the information that Jack left a generous tip, makes it almost inconceivable that the couple did not enjoy the food that was served. However, in the kidney stones colic illness script instantiation presented in Table 5.2, the finding that the patient, though he has just gone through a terribly painful attack, does not appear to be seriously ill, is highly typical for a leidney stones colic, though on the other hand, it is not thoroughly inconceivable that a patient who has just endured a kidney stones colic indeed appears to be very sick. As long as signs and symptoms are not by definition present in an illness, or necessarily implied by other signs and symptoms, they may be absent in any instantiation, however unlikely such may be. Thus, whereas the goal structure and social knowledge may make certain inferences almost inevitable in regular scripts, for illness script, this kind of inferencing may be much less compelling, and result in lower false alarms for omitted prototypical information. A third reason why omitted, but typical information in an illness script is not automatically inferred may be that a certain reluctance to infer unstated prototypical Consequences may even have a "survival value" in a medical profession. For example, in some cases, the absence of particular prototypical signs and symptoms may have meaningful consequences, e.g." for treatment or prognosis. Moreover, in an actual clinical situation, the possibility of a less probable disease than the one ranked highest in the differential diagnosis is always on the lurk, and it is important not to miss information pointing in this direction, even if it concerns the absence, rather than the presence, of certain prototypical signs and symptoms. Finally, physicians may also note the absence of certain typical signs and symptoms and incorporate this information in their mental base-rate probabilities for specific diseases. In light of these remarks, it may even be the case, though we have no actual evidence for it, that experienced physicians do actually tag unstated prototypical items as "symptom $X$ not mentioned", which might explain their relatively good memory performances for omitted information.

In summary, we think that the absence of an inclination to falsely recognize omissions in experienced physicians does not invalidate the notion of illness scripts; moreover, the finding that expert subjects do show poorer recognition memory for paraphrases than advanced students is consistent with the notion of more coherent and integrated knowledge structures, in which script-fitting information is easily encoded in a primarily meaning-based mode, in more experienced subjects.

In the foreseeable future, theories about the exact representation of (clinical) medical knowledge will continue to generate stadies. We have tried to find evidence for the illiness script theory, which states that clinical diagnostic knowledge 
can be represented as illness scripts; in addition, this theory makes specific predictions about the developmental course of these scripts. In a recognition study, we found evidence that this kind of medical knowledge indeed shows scripted properties, though predictions concerning the exact developmental course of the scripts could not be confirmed. The shortcomings of the present study alluded to in the previous sections may serve as cues for future research. For example, an effort to complement the present findings with memory performance after longer retention intervals could yield interesting results. In a similar vein, a recall study, which focuses especially on recall intrusions in illness scripts, is still lacking and wanting. Maybe we have taken the existing recall studies too much for granted, and are expert physicians not inclined to produce script-like recall intrusions at all. Finally, the developmental aspects of illness scripts again have been found elusive. Even in a recognition experiment, in which sensitive measures like reaction times are employed, expertise related differences are difficult to disinter. Though there are differences between memory performances of fourth-year students and experienced physicians, the foundations for many illness scripts seem to have been laid by the time the preclinical phase of the medical education is finished. Nevertheless, it still can be considered a challenge to devise experimental tasks to chart the developmental track of clinical medical know ledge. 


\section{CHAPTER 6}

\section{SUMMARY OF THE RESULTS AND GENERAL DISCUSSION}

\section{INTRODUCTION}

We will start this chapter by presenting a concise summary of the main results of the studies described in Chapters 2 through 5 . In the subsequent sections, the implications of the present findings for the representation and structure of medical knowledge and medical problem solving, for the development of this knowledge, and for medical education will be discussed. Finally, some suggestions for future research will be presented.

\section{SUMMARY OF THE PRESENT RESULTS}

In the previous chapters, four studies were described that aimed at investigating the development and structure of clinical medical knowledge, with a particular emphasis on diagnostic knowledge. In the introductory chapter, it was argued that this kind of knowledge might be represented as illness scripts, i.e., script- or schema-like structures, that are activated as a consequence of the information available in the clinical diagnostic situation, and that are instantiated with respect to information about the specific patient at hand. The expression "illness script" was first expanded upon by Feltovich and Barrows (1984), with the concept being relatively independently defined, i.e., as an elaboration of a template structure, without reference to the work of Schank and Abelson (1977) on scripts, nor to recent studies on schemas (e.g., Graesser \& Nakamura, 1982). Feltovich and Bartows (1984) were apparently inspired by recent efforts to implement medical expertise in an expert system, like the causal-rule based expert system MYCIN (cf. Clancey, 1983); hence, they conceived of illness scripts more as causal mental models than as "true" scripts. However, Feltowich and Barrows (1984) acknowledged the importance of Enabling Conditions (contextual factors and patient characteristics) causing a certain Fault (abnormality or malfunction in the human body), which in turn results in particular Consequences (complaints, signs and symptoms). Though this distinction between three script components has proven to be a highly fruitful idea, the causal model wiew of the illness script has not received that much empirical support: causal, biomedical reasoning turned out to be in general not a salient feature of expert problem solving, at least not in routine cases (cf. Boshuizen \& Schmidt, 1992; Clancey, 1983 ; Norman et al., 1989; Patel \& Groen, 1986a; Schmidt \& Boshuizen, 1993a). However, the fact that Feltovich and Barrows (1984) opted for the expression "illness scripts" has not remained without consequences; in the years following the introduction of the concept, a number of notions associated with the original Schank and Abelson' (1977) scripts, have been transferred to the medical domain, for example the idea that an illness script can be selected and instantiated in a diagnostic situation (cf. Schmidt et al., 1990).

The work of Hobus and his colleagues (Hobus et al., 1987, 1989, 1990) has revealed an important aspect concerning the development of the presumed illness scripts during the course of medical education. These authors report two important findings: First, that experienced physicians are much roore able to take En- 
abling Conditions into account in activating early diagnostic hypotheses than beginning physicians, and second, that experienced physicians are generally inchined to mention relatively more Enabling Conditions than their less experienced colleagues when asked to describe a prototypical patient with a specific disease. Based on these results and related work on medical expertise (e.g., Allen, Brooks, \&orman, 1988; Patel et al. 1989), a theory of the development of clinical medical knowledge was constructed (cf. Schmidt et al., 1990). This theory states that in the preclinical phase of the study, disease information is accumulated, and an enormous network of medical knowledge is constructed. During the clerkship phase, in which students walk the wards, this knowledge Is tuned to use in practical situations, and gradually full-fledged illness scripts develop. However, knowledge of Enabling Conditions is thought to show a considerable lag or delay compared to knowledge of the Consequences, as manifested by the relative inability of beginning physicians to exploit knowledge of patient contextual factors, and the relatively smaller contribution of Enabling Conditions in their descriptions of prototypical patients (Hobus et al, 1987, 1989). It is not quite clear why this developmental lag actually occurs, but the assumption is that students may be inclined to focus especially on the complaints, signs and symptoms associated with a disease, because these aspects are generally used to confirm diagnostic hypotheses and to alleviate diagnostic uncertainty, while Enabling Conditions are less conclusive in this respect. The tendency of medical textbooks to emphasize Consequences may also contribute to this developmental divergence of Enabling Conditions and Consequences. Furthermore, Schmidt and Boshuizen (1993a) and Schmidt et al. (1990) allow for a further specialization of clinical knowledge structures into "instance scripts", i.c., memories of individual patients that are thought to play an important role in expert future diagnoses of similar cases.

The studies described in this thesis were designed with two objectives in mind: First, to assess the "scripted" qualities of illness scripts, and second, to further investigate the proposed developmental course. As far as the first issue concerns, simply naming a hypothesized knowledge structure a script is obviously not sufficient for it to "be" a script; it is necessary to show that the features characteristic of "classical" scripts also apply to illness scripts. If experimental evidence can be gathered that is consistent with script-based predictions, this can be interpreted as showing the adequacy of the script representation for that particular knowledge. With respect to the second issue, if the development of knowledge of Consequences indeed precedes development of knowledge of Enabling Conditions, then this might be reflected by differences in a number of both process and performance measures, apart from the ones already investigated by Hobus and his colleagues.

In the study described in Chapter 2, particularly this second, developmental, aspect of illness scripts was addressed. On basis of the Hobus et al. (1989) study, in which subjects at different levels of expertise were asked to describe a prototypical patient with a particular disease, it can not definitely be concluded that less expert subjects actually lack the relevant knowledge; they may simply, for whatever reason, be less inclined to volunteer it in a recall task like the one employed by Hobus et al. (1989). To investigate whether this is the case, i.e., whether less experienced subjects are only less inclined to produce Enabling Conditions, rather than actually ignorant on this illness script component, every subject was presented with a scrambled case embedded in a pool of "noise" (i.e., irrelevant and case-inconsistent information), and asked to reconstruct a plausible case. It was hypothesized that if less experienced subjects lacked 
knowledge of Enabling Conditions, they would be less inclined to include Enabling Conditions into their case reconstructions; however, if they did select patient contextual factors, this might be taken as support for the assumption that they do possess this knowledge, and consequently that the Hobus et al. (1989) results should be contributed to access or activation deficits, rather than to the sheer absence of knowledge. In addition, to increase the sensitivity of the study, a third level of expertise, i.c., fourth-year students, was included, while in the Hobus et al. (1989) study only beginning and expert physicians participated.

The results of the study failed to show any differences in case reconstruction between the three expertise levels. Subjects at all three levels selected on the average approximately 13 statements; about one-third of these were Enabling Conditions. Additional manipulations, like asking subjects to add new statements or to discard a fixed number of selected ones, also did not yield any differences between the two groups. Moreover, $\chi^{2}$-analyses, applied to individual case statements, showed that subjects at different levels of expertise agreed to a large extent as to the information that was included in the case reconstruction. Some circumstantial evidence for the script representation was also gathered: information typical for the "hidden" disease was selected frequently, while irrelevant or inconsistent information was not included in most subjects' reconstruction. In summary, the general conclusion from these results was that it is highly probable that there are no differences between expert physicians and advanced preclinical students, as far as their ability to recognize case descriptions in general, and patient contextual factors in particular, is concerned. Nevertheless, this conclusion should be stated somewhat cautious, because only one disease was presented and hence, a case-specificity effect can not be excluded.

In order to investigate the development of illness scripts and the proposed differential role of Enabling Conditions and Consequences into greater detail, we decided to replicate and extend the Hobus et al. (1989) study. This extended replication study was described in Chapter 3 . Four different levels of expertise were included: fourth-year students, sixth-year students, interns (i.e., postgraduates in training as family physicians), and experienced family physicians. It was hypothesized that the number and/or proportion of Enabling Conditions produced in a free production task would increase with amount of experience, while simultaneously the number of biomedical elements mentioned would decrease. Furthermore, it was also predicted that experts' descriptions of a particular disease would be highly similar, regardless of the way they were probed, while especially subjects at intermediate levels of expertise would be sensitive to differences in instruction. Such sensitivity would suggest that intermediates are featured by a lack of integration of diagnostic knowledge into illness script structures. Finally, it was also investigated whether the number of patients seen with a specific disease, apart from expertise level in general, influenced the number and/or proportion of Enabling Conditions mentioned.

In this study, two different probes were used: subjects were asked to describe either a prototypical patient with, or the clinical picture of, each of 20 diseases, differing in frequency, seriousness, and organ system involved. The prediction was that expert physicians would always, i.e., regardless of the nature of the probe, be inclined to include some Enabling Conditions into their descriptions, while less expert subjects would only do so if probed to describe a prototypical patient, but not if asked to describe the clinical picture of a disease. Furthermore, it was hypothesized that especially the preclinical students would mention relatively much Fault-related, i.e., biomedical, information, while the importance of this illness script component would decline with increasing (practical) experience. 
To a large extent, these expectations were borne out. In general, up to the level of interns, more experienced subjects were inclined to mention more Enabling Conditions, but beyond, no effect of expertise level was found. However, there was a large difference between the two experimental probes: If asked to describe a prototypical patient, an inverted-U shaped relationship between expertise level and number (and proportion) of Enabling Conditions mentioned was found, while a request to describe the chnical picture of a disease resulted in a monotonically increasing relationship between these variables. This result was interpreted as in support of the idea that with increasing expertise level, Enabling Conditions become a more integral part of illness scripts; accessible also if the subject is not directly probed into this direction. Thus, it is not knowledge availability, but knowledge accessibility that can account for the superior performances of expert subjects reported in previous studies. Also in accordance with the predictions, the number of Fault-related items decreased with increasing experience, for both types of probes alike, and also up to the level of intems. Furthermore, it appeared to be practical experience with diseases, rather than expertise level in general, that was most strongly related to number of Enabling Conditions mentioned (and hence, to illness script development).

The aim of the studies described in the Chapters 4 and 5 was to zero in on the script aspects, rather than the developmental features, of illness scripts. The design of these studies was of a more quantitative nature, focusing at a relatively fine-grained level of analysis. The study described in Chapter 4 investigated the influence of expertise level, case typicality, and illness script component on case reading times and case probability estimates. Short computerized case descriptions, in which a patient was described with Enabling Conditions and Consequences that were either atypical or prototypical for the disease he or she was suffering from, were presented to sixth-year students and experienced family physicians. Subjects were instructed to read the cases as fast as possible, to decide whether or not the patient described in the case suffered from the presumed disease (which was announced prior to the case presentation), and to provide a probability estimate, expressed as a percentage between 0 and 100 , that the patient described in the case indeed had this disease. In addition, some NO-cases were included, in which a patient was described with a disease completely different from the one announced at the beginning of the case. Reading times of individual case statements were separately recorded, and average reading times of Enabling Conditions, Consequences, and complete cases were computed. It was hypothesized that experienced subjects would be faster than less experienced subjects, that prototypical case information would be processed faster than atypical case information, and that experienced physicians would be more sensitive to typicality of Enabling Conditions than advanced students. Furthermore, typicality would also contribute to the probability estimates, with the experts taking typicality of both Enabling Conditions and Consequences into account, while the nonexperts would focus mainly on Consequences in determining a probability estimate. However, though the expected effects of expertise level and case typicality on case reading times were indeed found, the influence of illness script component turned out to be additive, rather than interactive: the contribution of typicality of Enabling Conditions was similar to that of Consequences, at both expertise levels. As far as the probability estimates are concerned, indeed the expected interaction between expertise level and illness script component materialized, with the expert subjects showing somewhat greater sensitivity for typicality of Enabling Conditions than sixth-year students, though these latter subjects generally were also influenced by this factor. The overall conclusion was that 
expert knowledge structures can be adequately described as illness scripts, and that the development of these scripts involves both Enabling Conditions and Consequences.

The study presented in Chapter 5 was in essence a replication, modification, and extension of previous script based research, performed by Yelkovich and Walker (Walker \& Yekovich, 1984, 1987; Yekovich \& Walker, 1986) and Graesser and his colleagues (Graesser, 1981; Graesser et al., 1979; Graesser et al., 1980; Graesser \& Nakamura, 1982; Smith \& Graesser, 1981). In a recognition study, the inlluence of expertise level, actual textual presence, typicality, and illness script component on the accuracy of responses and the concomitant reaction times was investigated. In a study phase, fourth-year students, sixth-year students, and experienced family physicians were presented computerized case descriptions; the diagnosis was announced prior to the presentation, and all the subjects had to do was to read and understand the case. In a subsequent test phase, case statements were shown, and subjects had to decide, as quickly and accurately as possible, whether these statements had been literally presented in the associated case description, or not. These test statements differed in typicality, actual presence, and illness script component involved. Decision times and quality of the response (hit, false alarm, correct rejection, miss) were recorded. Concerning the accuracy of responding, it was hypothesized that memory discrimination would be better for atypical than for prototypical statements, and that this difference would increase with expertise level: expert subjects would be prone to falsely recognize prototypical, but unstated items. Concerning the speed of responding, it was predicted that experienced subjects would be faster than inexperienced subjects, that atypical statements would be responded to more quickly than prototypical statements, and that especially prototypical unstated items would show slow reaction times. An interaction with expertise level was also expected, with the expert physicians, though being generally fast, experiencing difficulty with prototypical unstated items. In addition, the reaction time data were compared to those of Yekovich and Walker (1986), in order to corroborate a proposed stage model of responding to scripted information.

The results of this study showed that subjects, and especially the more experienced ones, indeed made more errors of commission on prototypical unstated items than on atypical unstated items. It was also found that this effect could be attributed more to Enabling Conditions than to Consequences, at all three levels of expertise alike. As far as reaction times were concerned, all subjects showed script-consistent behavior, i.e., relatively long reaction times for unstated, prototypical information. This effect was also mainly due to the Enabling Conditions, at all three levels of expertise. Comparison of the present data with those of Yeko. vich and Walker (1986) showed similar results for hits and correct rejections, but whereas false alarms in this latter study apparently were a consequence of quick "recognitions", in the present study they seemed to be more the outcome of an extended deliberation, ultimately resulting in an inaccurate decision. A relevant additional result was that experienced physicians were strongly inclined to falsely recognize paraphrases, but not omitted information: Apparently, their memory for surface features of presented information quickly fades, but they are, in contrast with reports by other authors (e.g., Arkes \& Harkness, 1980), not more inclined than students to infer unstated signs, symptoms, or complaints.

In summary, the results indicate that illness scripts share the important behavioral properties (e.g. processing speed, recognition memory performance) of scripts and schemas, as defined and investigated in the relevant literature. Furthermore, it can also be concluded that, with increasing disease-relevant 
experience, subjects' illness scripts become more and more integrated and consolidated. The differential role of Enabling Conditions and Consequences in script development materialized mainly as differences in accessibility of scripted structures in a recall task as a consequence of probing; in an illness script recognition task, Enabling Conditions and Consequences seem to behave in a highly similar fashion for both students and experts.

\section{IMPLICATIONS FOR THE REPRESENTATION OF MEDICAL KNOWLEDGE AND MEDICAL PROBLEM SOLVING}

How can the representation of medical clinical knowledge best be described? In the previous chapters, four options were addressed, either theoretically or in experiments, or both. These options were: mental models of medical knowledge, prototypical patients, illness scripts, and representations of individual cases. First, it should be emphasized that physicians probably dispose of medical knowledge corresponding to all of these four types of representation: They are able to reason about diseases and cases in order to explain complaints, symptoms, and signs (ef. Feltovich et al., 1992; Hassebrock \& Prietula, 1992); they have, for many diseases, a relatively clear-cut image of the prototypical patients associated with these diseases (cf. Chapter 3); their knowledge shows script-like properties (cf. Chapter 4 and Chapter 5), and they have memories for previous cases, even of many years' standing (cf. Hassebrock \& Prietula, 1990; Van Rossum et al., 1990).

In this thesis, we have tried to make a case for an illness script representation of medical (clinical) knowledge. First, many previous studies have shown that elaborate reasoning is generally not a salient feature of experienced physicians' diagnostic performances, at least not in routine cases (e.g., Norman, Brooks, \& Allen, 1989; Patel \& Groen, 1986a; Schmidt \& Boshuizen, 1993a). Experts" elaborate reasoning is associated with difficult diagnoses, and a relatively high proportion of errors (Norman, Brooks, \& Allen, 1989). Second, though expert physicians in general have no difficulty in describing a prototypical patient with a particular disease, prototype representations are not adequately equipped to deal with the underlying time dimension of medical cases (however, for a different opinion, see Barsalou \& Sewell, 1985). The finding that subjects tend to recall scrambled case information in accordance with the standard patient presentation order (Coughlin \& Patel, 1987) does not point to a knowledge structure based on prototypicality; the features of a prototype do not have that kind of natural order. Prototypes also do not offer opportunities for representing the differential role of Enabling Conditions and Consequences. Third, as yet there is also no conclusive evidence that experienced physicians' diagnosis relies heavily on a "chorus of individual previous instances," stored in memory. It seems likely that repeated experience with similar cases results in knowledge structures from which the irrelevant aspects of the patients are filtered out; otherwise, physicians would be easily induced to activate a large number of highly unlikely diagnostic hypotheses. We venture the hypothesis that the use of memories for previous cases reported in the literature may sometimes have been an artefact of reinstating the original context in which the previous case was presented (cf. Godden \& Baddeley, 1975). For example, physicians participating in an experiment might be reminded of a previous session, in which the prior case was presented, when again faced with the experimental equipment; seeing a corresponding case in daily practice probably may not lead to renewed activation. However, it is feasible that for rare diseases, for which (initially) no appropriate 
illness script is present, physicians will use memories of concrete prior cases. Similarly, particularly highly salient Enabling Conditions may also result in a lasting memory representation of a case, even if the actual Fault is relatively common; for example, Van Rossum et al. (1990) found that - the terminology is ours - "curious Enabling Conditions" indeed led to reactivation of the prior diagnostic hypothesis when a new case with the same background was presented, but not to the point of completely overnuling alternative hypotheses. In summary, though patient memories may play an important role in non-routine situations and for some remarkable patients, it seems reasonable to assume that after having seen two or more cases with a specific disease, a more general structure, i. c. an illness script, will be formed.

Findings that causal models, prototypes, and instances all have drawbacks when it comes to representing expert medical knowledge, do not in itself constitute evidence for the psychological viability of illness scripts. On purely theoretical grounds, illness scripts have to be different from "classical" scripts, for they imvolve neither goals nor actors ${ }^{20}$. Nevertheless, it might be argued that the underlying time dimension provides a basis for describing diseases as illness scripts, with the illness script components (Enabling Conditions, Consequences, and Course/Therapy) playing the role of script scemes, and the individual context factors, signs, symptoms, and complaints playing the role of script actions and concepts. The results of the experiments described in the Chapters 4 and 5 have shown that the presumed illness scripts indeed show similar behavior as classical scripts, such as slow reading times for atypical information and a high proportion of false recognitions for paraphrased information. However, one important finding could not be replicated: the tendency of subjects to falsely recognize unstated, i.e., omitted, but typical, script information. In this respect, illness scripts appear to be truly different from classical scripts. In the discussion section of Chapter 5, some reasons were mentioned why illness scripts are different from classical scripts in this respect, the most important of these being that disease complaints and symptoms do not necessarily imply each other, while actions in "classical" scripts often do. How detrimental is this finding to the idea of illness scripts? If scripts are relatively broadly defined, as general event sequences, then there is no reason to deny illness scripts their script status. The alternative would be to assume a category structure of disease knowledge based on prototypicality; as we have already argued, prototype representations are unable to cover a number of relevant findings with respect to disease knowledge. However, in recent years, category theories are no longer restricted to perceptual. ly based categories, but allow for causal underlying structures (cf. Medin \& Ortony, 1989); as such, they may gradually be able to incorporate script-like properties (and vice-versa, cf. Barsalou \& Sewell, 1985). In summary, based on the present results we think that illness scripts should be placed somewhere between prototypes and "classical" scripts, be it somewhat closer to the latter ones.

As far as the representation of medical knowledge is concerned, some more basic issues have to be dealt with here. The results of the studies presented in Chapters 2 and 3 , in combination with previous data of Hobus et al. (1987), show that subjects at intermediate levels of medical expertise (sixth-year students, interns) do possess knowledge of Enabling Conditions, but are still unable to use

20 In our conceptualization of the Illness script, neither the physician, nor the patient can be considered a "irue" actor in the sense of Schank and Abelson (1977). Similarly, the "goal" of an illness script (i.e., correct diagnosis or treatment) can also not be put on a par with the socially defined goals of the "classical" scripts. 
this knowledge in a diagnostic situation. It was concluded that in subjects at these levels of expertise, Enabling Conditions are not yet integrated into illness script structures. But what does this conclusion mean? Throughout this thesis we have implicitly - and in Chapter 5 even explicitly - adhered to a network based view of knowledge representation, in which scripts are embedded as specific sub-networks (cf. also Yekovich \& Walker, 1987). In such a network, concepts are represented by nodes, and relations between concepts by links between nodes. These links enable a process of spreading activation, by which nodes may activate or inhibit each other. Two important features of such links are association strength and directionality. The connections between two nodes may be strong or weak, in either direction. Poor integration of Enabling Conditions into illness scripts can possibly be modeled as unbalanced links between nodes: the script header (diagnosis) may be strongly connected with several important Enabling Conditions and Consequences, while in the opposite direction, especially the Enabling Conditions are only weakly connected with the script header, for example, as a consequence of a "fan effect" (cf. Anderson, 1983). This situation may apply to subjects at intermediate levels of expertise: If they are presented with a diagnosis, they have no difficulty in mentioning the associated Enabling Conditions; however, the diagnosis itself will be activated mainly by the Consequences; the presence of Enabling Conditions may add little activation to that provided by complaints, signs and symptoms. Thus, if the diagnosis does not become part of working memory (activated long-term memory) as a result of activation of Consequences, Enabling Conditions may be of little help.

The gradual integration, with increasing expertise level, of Enabling Conditions into illness scripts might also be modeled by a production system architecture (cf. Anderson, 1983, 1993). In fact, the inherent asymmetry of production systems, provided by the directionality of production rules, may be particularly apt to model this situation. For example, with the diagnosis on the condition side of a production (i.e., explicitly mentioned), it may be easy to activate both relevant Enabling Conditions and Consequences. On the other hand, if the purpose of the production rule is to activate the diagnosis, inclusion of Enabling Conditions into an already complex condition consisting of Consequences may not increase the probability that this actiwation is achieved by firing of the production rule. This may be the case for intermediates; when they gradually become experts, the Enabling Conditions included in the condition side of the productions may tune the production rules to activation of more specific diagnoses than on basis of the Consequences alone.

However, presently we are especially concerned with a more psychological description of the diagnostic process. The core, and in our view also the most interesting stage of this process consists of the quick activation of a limited number of illness scripts as a consequence of the information available in the initial stage of the consultation or visit. This process of illness script activation proceeds automatically, for experts and non-experts alike. The critical difference between experienced and less experienced subjects concerns the appropriateness of the activated illness scripts; experts are more likely to activate the correct script than novices, because they are able to use information about Enabling Conditions, available in the diagnostic situation. This process of automatic script activation may be supplemented by some more controlled, additional search. For example, Weber et al. (1993) found evidence that physicians continue to generate diagnostic hypotheses until at least one serious alternative that can, be it with a low likelihood, account for the present symptoms and complaints, is found. If this diagnostic hypothesis is a standard alternative, given the Enabling Conditions and 
the complaint, it may be automatically activated; if it is not, then it may have to be activated by a controlled search. Once the usually small, initial set of hypotheses has been formed, subjects are highly reluctant to generate new hypotheses fur ther downstream the process of diagnostic problem solving (cf. Elstein et al., 1978; Wagenaar, 1987); new symptoms are used to test current hypotheses, not to generate new ones. People are often more inclined to neglect or "explain away" disconfirming symptoms than to reconsider their current hypothesis, at least if they can not think of a better one. Thus, it is not unusual for people to generate hypotheses they know do not account for all of the relevant facts. Up until recently, these kinds of behavior were interpreted as reasoning errors (e.g., a confirmation bias, cf. J. Evans, 1989); however, we think they can be more appro priately conceived of as efforts to reach a local maximum in a problem solving situation in which the optimum solution may be beyond reach. Subjects fail in a diagnostic problem solving situation because they are unable to activate an illness script that fits the present case; they stick to their current hypothesis as long as they can not think of a better one. With increasing experience, the situation gradually improves, because a richer base of illness scripts develops, and the individual scripts become more tuned toward the situations in which they are potentially applicable. After sufficient experience, problems that were initially hard may become routine, i.e., the appropriate illness script is activated almost immediately. Apart from an increased likelihood that the correct diagnosis will be found, a large repertory of well-tuned scripts may also have an additional advantage: it may free time and resources to deal with situations that are less familiar. A physician for whom a large proportion of cases is of a routine nature, may be able to spend more time on the non-routine patients, or on the non-routine aspects of otherwise routine cases. For example, an experienced physician may be able to recognize that the presenting complaint of a patient is only a pretext and use deliberate reasoning and problem solving to find out the "true" story behind it, while a less experienced physician has to invest all available resources to diagnose the "surface" problem. From this example it may also be inferred that the illness script theory certainly does not pretend that all knowledge used by experts in a medical diagnostic situation is embedded in full-fledged illness scripts, but only that physicians dispose of scripted disease knowledge for a large number of relatively frequently occurring diseases.

\section{THE DEVELOPMENT OF MEDICAL KNOWLEDGE}

In the previous chapters, as well as in the preceding sections, a great deal of information has already been provided about the development of medical knowl edge. In general, we adhere to the model proposed by Schmidt and Boshuizen and Boshuizen and Schmidt in the early 1990s. The illness script is a central aspect of this model. Clinical knowledge is assumed to be represented as illness scripts; this applies to experts as well as to less experienced subjects. "The major difference is that expert illness scripts are more finely tuned towards practical situations; this is, at least partly, a consequence of the integration of Enabling Conditions in the scripted structure, enabling the experts to quickly activate ap propriate illness scripts in many situations. Experts Consequences may also be better tuned than those of novices (cf. Hobus et al., 1988). As such, the development of illness scripts can be considered a form of weak restructuring of knowledge (cf. Vosniadou \& Brewer, 1987). Experts represent more and/or different 
relations between concepts than do novices, or, as seems to be the case for illness scripts, directionally more balanced relations: though advanced students are able to activate knowledge of Enabling Conditions given a diagnosis, the other way round is not yet passable by these subjects. A reason for this imbalance in the relations between concepts may be that Enabling Conditions are usually of a more general nature than Consequences. Thus, whereas a combination of a few Consequences often points unambiguously in a certain direction, for Enabling Conditions, this may much less often apply: They constrain the search space, but usually not to the point of excluding all but a few hypotheses. This is particularly the case for Enabling Conditions like age, sex, occupation (a few exceptions notwithstanding), environment, life style, and risk factors; these variables are probabilistically related to a large number of diseases. On the other hand, Enabling Conditions like previous diseases, use of medication and hereditary influences are often of a much more specific nature, and hence may play a role equivalent to that of Consequences. Neither the Hobus et al. studies, nor the present studies distinguished between different scopes of Enabling Conditions and Consequences; consequently, a suggestion for future research might be to replicate the Hobus et al. (1990) study to investigate the influence of specific versus general Enabling Conditions and Consequences.

Though no conclusive evidence has been gathered on the topic, there are some indications that the development of illness scripts and the concomitant integration of Enabling Conditions into these scripts follow a course relatively independent from the acquisition of biomedical knowledge, but contingent upon experience with actual patients. For example, we found (Chapter 3 ) that subjects' inclination to mention Enabling Conditions is associated to the number of patients they have seen with a specific disease, but not to medical experience in general. Weber et al. (1993) also report that medical experience has no independent effect on hypothesis generation beyond the "having seen of similar cases before." Furthermore, the contribution of biomedical knowledge in descriptions of patients or clinical pictures asymptotes to about zero lang before the full integration of $\mathbb{E n}$ abling Conditions takes place (cf. Chapter 3 ); this also suggests a relatively independent development of illness scripts, at least as far as the final stages of this development are concerned. In fact, it may be theoretically possible that illness scripts develop without knowledge of the underlying biomedical model, just like subjects in the study of Ahn et al. (1992) were able to learn the details of a "potlatch" ceremony, without knowing the reasons behind the actions that had to be performed. Similarly, physicians in non-western cultures, physicians in the middle ages, and physicians working according to principles of alternative medicine, may have illness scripts that are either not tied at all to an underlying biomedical model, or based on an alternative, maybe even incompatible, model. Indeed, even regular, late 20th century physicians in Western Europe or America may have idiosyncratic illness scripts (cf. Schmidt, Norman, \& Boshuizen, 1990), that may not be properly embedded in regular biomedical knowledge. Nevertheless, it is likely that most students do employ biomedical knowledge in developing illness scripts, if only to be able to explain why particular Enabling Conditions and Consequences are (inter-)related, in addition to knowing that they are part of the same disease (i.e, an aspect of their clinical knowledge). If, in a diagnostic situation, an appropriate illness script can be quickly activated, no biomedical knowledge is necessary to establish a diagnosis; if this process fails, subjects will try to find a diagnosis by explaining, initially on basis of clinical knowledge, but if this is also lacking, on basis of biomedical knowledge, the possible common origin of the complaints, signs and symptoms. 
In this thesis we have focused mainly on intra-individual cognitive aspects, and implicitly we have considered diseases as fixed, objective entities. However, we have to address the issue of the interaction between the "external world" and the human cognitive system here. A particular prominent aspect of this interaction concerns the nature of signs and symptoms. Throughout the previous chapters, it has been implicitly assumed that signs and symptoms are objectively perceptible and unchanging entities. However, there is evidence (cf. Bransford, Franks, Vye, \& Sherwood, 1989; Lesgold, 1984) that experts may perceive quite different features than novices. Thus, the problem may not be that it is difficult for inexperienced subjects to learn or remember rules like: "diagnosis $X$ is applicable if the patient is moderately depressed" - the problem is that perceiving a feature like "moderately depressed" is the result of a complex pattern recognition process in an expert, while the rule does not provide clues for a nowice how to recognize this symptom. In addition, some features may only become perceptible if the illness script is already activated. Lesgold (1984) points to such a situation in which the triggering of the atelectasis (a pulmonary affliction) script on basis of some features of a chest X-ray, leads to the perception of the other features of this condition as well. Though the importance of this observation may be particularly high for complex visual domains like radiology and dermatology, they may sometimes also play a prominent role general medicine. A related phenomenon is the interaction between signs or symptoms as a consequence of the condition of the patient. For example, heart sounds of highly overweight patients may differ considerably from those of subjects with average weight. An important aspect of diagnostic expertise development may be a growing sensitivity for this interdependence between features. In summary, it should be emphasized that to consider signs and symptoms as objectively perceptible, unchangeable, and for subjects at all expertise levels alike, is in fact a simplification.

\section{IMPLICATIONS FOR MEDICAL EDUCATION}

Our experimental results do not provide direct suggestions for medical educational practice. However, especially in light of previous research, some tentative recommendations can be formulated.

First, how can education foster the development of illness scripts? In the study described in Chapter 3, we found that the development of illness scripts is associated with subjects' amount of experience with actual patients who suffer from the diseases in question. Thus, it is important to provide students with opportunities to see patients. In this respect, it is probably better for students to see many patients for a short time, than to delve deeply into a few cases. To foster illness script development, the cases presented to students should be as representative as possible for the specific illness scripts (cf. Bordage, 1984; Murphy \& Wrught, 1984; Van Rossum et al., 1990). Cases with much "noise" should be avoided, especially patients with an impressive, but irrelevant context (Van Rossum et al., 1990), because this may cause students to develop a wrong image of the Enabling Conditions. An additional reason why the use of actual patients should be recommended is that since patient problems are the future context in which medical knowledge is used, why not also teach it in this context? (cf. Godden \& Baddeley, 1975; Norman, 1988). This notion is also implicitly present in the recommendation of Elstein et al. (1978), who advocate to provide students with repeated exercises in knowledge application for a similar range of problems as they will be experience in the future. 
However, as for practical reasons the opportunities for students to see and examine patients probably will remain limited, it may make sense to encourage them to gather as much knowledge, especially clinical knowledge, about diseases and patients as possible, not only by studying textbooks, but also by watching vildeotapes, attending patient demonstrations, etceteras. Though an extensive theoretical clinical knowledge base it not sufficient for developing full-fledged illness scripts, it may be very helpful, because students may develop relatively welldefined expectations concerning what they will see when faced with concrete patients. Thus, it is likely that they can profit to a larger extent from their actual clinical experiences, if the theoretical background of their (future) illness scripts is already present.

On the other hand, it does not seem to make much sense to teach students explicitly the strategy to use Enabling Conditions in trying to diagnose a case; the integration of Enabling Conditions into illness scripts is something that takes much time and experience. The use of Enabling Conditions in the initial phase of a diagnostic situation is not under voluntary control. Furthermore, especially advanced students already possess this knowledge, and may know that it is important, even though they are unable to use it.

Similarly, it should also not be recommended to teach students hypothesis generation, as, for example, Benbassat \& Shiffman (1976), advocate. Prolific generation of hypotheses is not characteristic of expert behavior; addition of a new hypothesis (or substitution of a rejected hypothesis by a new one) is only useful if this more recently activated hypothesis is qualitatively better than the best of the old ones. What we do recommend is that students be stimulated to generate reasons why their diagnostic hypotheses may be incorrect in a certain case (cf. Wagenaar, 1987). As already mentioned, people tend to stick to their current hypothesis, even if they know or may expect that it can not be correct, as it flies in the face of the empirical evidence. However, to ignore the evidence may be more comfortable than to end up with no hypothesis at all. In a medical diagnostic situation, such behavior may be manifested by the physician implicitly denying part of the information provided by the patient (e.g., "You really do not have a hoarse cough?"), not necessarily because he suspects the patient to be insincere, but because the information is inconsistent with the, in his eyes, currently most likely diagnostic hypothesis. Students should be made aware of this kind of behavior, which may impair the quality of their diagnostic performances, apart from being unfavorable to the quality of the doctor-patient relationship.

What options are available if the student or physician gets stuck in such a situation? The illness script theory does not provide any clues as to how to find a way out of such a situation. Though it may intuitively be assumed that a search for additional information may lead to a solution, according to Elstein et al. (1978), insufficient information is usually not a critical diagnostic bottleneck. Stubbornly continuing to gather data, however remote they may be from the actual problem of the patient, is not a good strategy: The probability that the appropriate illness script will be actiwated is small, and in practical situations, it is apt to lead to costly and unnecessary diagnostic procedures. Rather, a physician should rely on good amnestic interview skills, because these are the most valuable and least expensive tools he has at his disposal; a fact already acknowledged by Wilkins (1970), who remarks that a good history should be given $80 \%$ of the weight in a diagnosis. If the correctly performed anamnestic interview does not provide clues as to what might be the most likely illness script, neither students nor experienced physicians should be reluctant to consult medical handbooks or colleagues in order to arrive at the appropriate diagnosis. 
A topic that has received considerable attention in the literature on medical expertise and medical education is the use of Bayesian statistics and medical decision theory in diagnosis and treatment (e.g." Biela, 1986; Camerer \& Johnson, 1991; Christensen-Szalanski \& Bushyhead, 1981; Hamm, 1988; Kuipers, Mascovich, \& Kassirer, 1988; Schwartz \& Griffin, 1986). From the present point of view, two things can be said about this. First, it surely is important that the episodic knowledge base of a physician - i.e., the pool of instances his illness scripts are based upon - is representative for the population frequencies (Weber et al., 1993). Experience with the "wrong" type of cases for a disease, as well as with the "wrong" diseases for a population, may result in a systematic bias in illness scripts. However, quite another question is whether it is effective to explicitly teach students and physicians to take Bayesian principles into consideration in arriving at a diagnosis. It should be clear from the previous discussion that the initial activation of illness scripts in a diagnostic setting is not a process under voluntary control. Thus, explicitly applying Bayesian principles in the early stages of diagnostic problem solving, is not possible. Nevertheless, there is evidence that "Bayesian-like" principles are implicitly incorporated into the activation of illness scripts. For example, Christensen-Szalanski and Bushyhead (1981) found that, though it is difficult for physicians to incorporate explicitly presented base-rate information into their diagnostic judgements, they do use apriori probabilities obtained from experience. This finding suggests that it indeed might be more sensible to provide students with cases in frequencies that are as representative as possible for the future patient population they will encounter, than to let them solve problems in which base-rate probabilities have to be explicitly used. On the other hand, it may be useful to teach some general principles in this respect, like the difference between representative features and diagnostic features of diseases (Klayman \& Brown, 1993), or, as Hodgkin (1984) recommends, awareness of the fact that rare symptoms of common diseases occur more frequently than common symptoms of rare diseases. However, this kind of knowledge is probably more relevant in deciding between two competitive, simultaneously activated illness scripts, than in the process of illness script activation itself. In general, we think that the illness script theory and medical decision theory complement rather than contradict each other (cf. also Patel \& Coughlin, 1985): The former theory is a descriptive theory about the way clinical diagnostic knowledge is represented and activated, the latter a normative theory about making decisions in situations that are characterized by a degree of uncertainty.

\section{SUGGESTIONS FOR FURTHER RESEARCH}

In the discussion sections of the Chapters 2 through 5, a number of recommendations and suggestions for future research have already been proposed. For example, the script recognition study in Chapter 5 might be extended to longer retention intervals and to recall measures, particularly in order to solve the discrepancy between the results of this study and previous research, which reported that subjects do infer unstated, but script or schema typical information. Furthermore, an experimental distinction between general and specific Enabling Conditions and Consequences might yield interesting results. For example, it may be found that general Enabling Conditions, or background information in the sense of Weber et al. (1993) (like age, sex, occupation, and environment), may play a different role compared to more specific information, like medical history, current medication, travelling to tropical countries, and hereditary factors. 
In Chapter 5 , it was hypothesized that the failure to find a false recognition effect of omitted signs and symptoms should be attributed to the absence, at least between separate symptoms, of a causal structure in illness scripts. Therefore, it would be interesting to compare illness scripts with classical scripts that also lack a causal structure, like a circus ring performance program (cf. Bower et al., 1979). In a circus performance, the appearance of lions, for example, does not imply the appearance of clowns, and vice versa; thus, in contrast to the restaurant situation, memories of a text about a circus program will contain no causal cues about the actual presence of the different acts. Another example of such scripts can be found in a study of Ahn et al. (1992), in which subjects had to learn a difficult script (the "potlatch ceremony") for which no causal or goal structure could be invoked. If these types of scripts would show the same memory phenomena as the illness scripts in the study in Chapter 5, particularly as far as the false recognition data of omissions are concerned, then this would provide additional support for the illness script theory. It would also indicate that illness scripts can in principle operate without knowledge of an underlying model, because the potlatch ceremony - and probably the circus performance, too - lack such a model. This would imply that illness scripts, at least in principle, can be learned almost without any underlying knowledge of the medical basic sciences.

Another question concerns the initial development of illness scripts. The results of the studies described in the present thesis show that sixth-year students already posses relatively well-developed illness scripts, while even fourth-year students may have script-like knowledge structures for some cases. It would be interesting to investigate whether preclinical students are inclined to construct illness scripts in an effortful manner, for example by inserting selfgenerated explanations into their beginning scripts. To a certain extent, this would be equivalent to the behavior of subjects in Bartlett's (1932/1954) experiments with the mysterious, difficult to comprehend "War of the Ghosts" story. By generating explanations for the different actions in this story, subjects were able: to remember it, though with a considerable distortion towards a more ordinary scripted story. In a similar vein, it is possible that preclinical students try to comprehend a difficult case, with many apparently disparate signs and symptoms, by generating explanations for the coherence of the features. Parts of these explanations may subsequently become part of the illness script structure, and hence intrude during recall, or lead to false recognitions in a recognition task. In general, we think that especially in the early stages of illness script development, biomedical explanations of the reasons why certain symptoms or patient characteristics are related to each other play an important role in interconnecting the elements of illness scripts; in later stages, firm associations between the constituent parts of these scripts will establish, and consequently the role of the underlying biomedical explanations decreases. If a physician is in doubt whether a certain illness acript is applicable, he will tend to use clinical, rather than biomedical, reasoning to solve the problem.

Still another issue, already addressed in Chapter 3 but in need of more research, concerns the question of the inclusion, and possible role, of a fourth illness script component, i.c., Course \& Treatment. Though this component may be of little interest for the initial stages of the diagnostic process, it may have important implications for later stages. The results of Chapter 3 suggest that knowledge of this component is acquired in a relatively late stage of expertise development. Similar studies like the ones described in Chapter 4 and 5 might be used to investigate whether knowledge of the expected course of a disease and the treatment eventually become part of the scripted structure. 
However, though these studies could reveal interesting aspects of illness scripts, we think that only more fine-grained models, in which the basic elements of cognition are explored, can ultimately indicate why experienced physicians are so much more able to generate accurate hypotheses on basis of scarce information, than less expert subjects. Though this is of course not the only feature of medical expertise (e.g., confirming a proposed diagnosis, choosing and applying a treatment, advising a patient, and so on, are also important aspects), we think it is an extremely important one. Expert diagnostic knowledge is organized in a way that enables experienced physicians, faced with a patient, to quickly recognize in many cases a small number of possible diagnostic hypotheses, even to the point of already "knowing" at that moment which disease the patient suffers from. At the behavioral level, especially if qualitative measures are employed, it will be difficult to find cues to explain this capability. As Hofstadter (1985) argues, the most interesting aspects of cognition take place at the level of $100 \mathrm{msec}$ or less - "the time it takes to recognize your mother." For most people, it will make simply no sense to ask how or why they recognize their mother - then why ask experienced physicians how or why they recognize that a patient has a disease the physician may have seen almost as often as his or her mother? Real progress in expertise research can only be expected from studies that go beyond - indeed, way beyond - the purely descriptive level. In this thesis, we have tried to make a small step towards a more explanatory model, to disclose something of what is generally called the "clinical look." 



\section{SAMENVATTING (SUMMARY IN DUTCH)}

\section{De ontwikkeling en functie van ziektescripts: Een onderzoek naar de structuur van medisch-diagnostische kennis}

In dit proefschrift wordt verslag gedaan van een viertal onderzoeken die uitgevoerd zijn met als doel een nader licht te werpen op de representatie en ontwikkeling van medisch-diagnostische kennis. Tezamen kunnen deze onderzoeken geplaatst worden in het kader van modellering en theorievorming met betrekking tot de ontwikkeling van expertise. In Hoofdstuk 1 wordt allereerst een historisch overzicht gegeven van het onderzoek in de psychologie dat aanzet heeft gegeven tot de huidige belangstelling voor de aard en ontwikkeling van expertise. Beargumenteerd wordt dat de wortels van het huidige expertise-onderzoek gezocht moeten worden in met name het onderzoek naar probleemoplossen en naar de wijze waarop kennis gerepresenteerd kan worden. Een aantal theorieën met betrekking tot beide onderwerpen wordt kort besproken. Een belangrijke plaats wordt ingeruimd voor het onderscheid tussen modellen die ervan uitgaan dat kennis al redenerend en elaborerend wordt toegepast, en modellen die de nadruk leggen op kennis als kant-en-klare pakketjes, die min of meer automatisch als geheel toegankelijk zijn tijdens het probleemoplossen. Uit eerder onderzoek is gebleken dat het laatste het geval is met de diagnostische kennis van ervaren artsen, zeker in routinesituaties: deze kan gerepresenteerd worden in de vorm van ziektescripts. Alhoewel Feltowich en Barrows (1984) de eersten waren die het ziektescript als kennisstructuur nader hebben uitgewerkt, en het begrip een relatief onafhankelijke status hebben gegeven, wordt in het huidige proefschrift gekozen voor een andere invalshoek, waarbij het ziekteseript veel sterker gekoppeld wordt aan het "klassieke" script, zoals dat gedefinieerd en uitgewerkt wordt door Schank en Abelson (1977). In deze zienswijze worden ziekteseripts in eerste instantie geactiveerd door binnenkomende diagnostische informatie, en vervolgens geinstantieerd met behulp van de verdere gegevens van de betreffende patiënt of casus.

Volgens Feltovich en Barrows (1984) bestaat een ziektescript uit drie belangrijke componenten: de Enabling Conditions (factoren uit de context van de patiënt die van invloed zijn op de kans dat iemand een bepaalde ziekte krijgt, zoals leeftijd, geslacht, medische voorgeschiedenis, erfelijkheid en risicogedrag), de Fault (een biomedisch model van het zich in het lichaam afspelende zielkteproces), en de Consequences (de klachten, symptomen en verschijnselen waartoe dit ziekteproces aanleiding geeft). Dit onderscheid is voor de praktijk van groot belang gebleken. In tegenstelling tot wat door velen, waaronder Feltovich en Barrows (1984) zelf, aangenomen wordt, is het niet zo dat het diagnostisch proces bij ervaren artsen gepaard gaat met een biomedische redenering waarin de verschillende componenten van het ziektescript met elkaar in verband worden gebracht. Ervaren artsen activeren vaak al op grond van enkele belangrijke Enabling Conditions en de ingangsklacht van de patiẻnt één, of een klein aantal, kwalitatief goede diagnostische hypothesen in de vorm van ziektescripts (cf. Hobus et al, $1987,1989,1990)$. De overige diagnostische activiteiten, zoals lichamelijk onderzoek, zijn dan voornamelijk gericht op het bevestigen van deze eerste indruk, of het kiezen uit een klein aantal mogelijkheden. Minder ervaren artsen en studenten moeten wel vaak hun toevlucht nemen tot het langs biomedische weg "aan elkaar redeneren" van de verschillende informatie-onderdelen uit een casus.

Deze, en aanverwante, bevindingen hebben geleid tot een theorie over de ontwikkeling van klinisch-medische kennis (Schmidt, Norman, \& Boshuizen, 1990). 
Deze theorie stelt dat in de preklinische fase van de geneeskunde-studie studenten woornamelijk bezig zijn met het vergaren van ziektekennis en het opbouwen van een gigantisch netwerk van, met name biomedische, kennis. Gedurende de coschappen, wanneer ze in de kliniek rondlopen, wordt deze boekenkennis toegespitst en afgesternd op gebruik in praktische situaties. Geleidelijk aan ontstaan volwaardige ziektescripts, zij het dat dit proces geruime tijd in beslag neemt en zeker nog niet voltooid is op het moment dat het basisarts-diploma wordt behaald. De bevinding dat net afgestudeerde basisartsen veel minder goed dan experts in staat zijn om gebruik te maken van gegevens uit de context van de patiënt (Enabling Conditions), wijst er op dat de ontwikkeling van deze component van het ziektescript pas in een relatief laat stadium wordt voltooid.

Van de vier experimenten waarvan in de hoofdstukken 2 tot en met 5 van dit proefschrift verslag wordt gedaan, zijn er twee rechtstreeks gericht op deze theorie van de ontwikkeling van medische kennis: er wordt geprobeerd aanvullende ondersteuning te vinden voor de ziektescript-theorie en de relatief late ontwikkeling van kennis met betrekking tot Enabling Conditions. De andere twee experimenten hebben als hoofdthema de vraag in hoeverre ziektescripts vergelijkbaar zijn met de Schank en Abelson (1977) scripts. In de jaren volgend op de introductie van het script-begrip zijn een groot aantal onderzoeken uitgevoerd waarin de bruikbaarheid en theoretische levenswatbaarheid wan het begrip getoetst werd. Indien experimentele evidentie kan worden gevonden voor de veronderstelling dat ziektescripts tot dezelfde geheugenprestaties en verwerkingspatronen aanleiding geven als klassieke scripts, dan vormt dit eveneens ondersteuning voor het nut van het (theoretische) concept ziektescript.

In Hoofdstuk 2 worden de resultaten beschreven van een scrambled case reconstruction study. Achtergrond van dit onderzoek vormde de vraag of het gevorderde studenten en minder ervaren artsen ontbreekt aan kennis van Enabling Conditions, of dat ze in principe wel beschikken over deze kennis, maar niet in geïntegreerde vorm in ziektescripts. Als het eerste het geval is, dan zullen minder ervaren proefpersonen ook slechter in staat zijn deze informatie te herkennen, zelfs als ze er als het ware met hun neus op geduwd worden; beschikken ze wel over de relevante kennis, maar is deze onvoldoende geintegreerd in ziektescripts, dan zullen ze onder bepaalde omstandigheden er we! gebruik van kunnen maken, bijwoorbeeld wanneer zij rechtstreeks wordt "aangeboord", terwijl zij onder andere omstandigheden ontoegankelijk kan zijn. Om dit te onderzoeken werd aan vierdejaars-studenten, zesdejaars-studenten en ervaren huisartsen een scrambled case reconstruction taak voorgelegd. De bedoeling van deze taak was dat de proefpersonen zelf een casus samenstelden (reconstrueerden) op basis van een verzameling losse brokstukken casus-informatie. Deze informatie, die bestond uit korte zinnetjes afgedrukt op in totaal 48 kaartjes, had betrekking op zowel de achtergrond en context van de patiënt, als op diens huidige klachten en symptomen. Sommige kaartjes bevatten "echte" casusinformatie, andere slechts "ruis", en weer andere zelfs informatie die strijdig was met de bedoelde ziekte. De scrambled case was zodanig samengesteld dat het herkennen van de verborgen ziekte (i. c., de ziekte van Pfeiffer) op zichzelf niet moeilijk bleek, ook niet voor de vierdejaars-studenten; de vraag was dan ook niet of ervaren artsen beter in staat zijn een ziekte te herkennen temidden van een hoeveelheid ruis, maar of zij meer dan studenten geneigd zouden zijn Enabling Conditions op te nemen in hun casus-reconstructie. Als dit laatste het geval is, dan wijst dit erop dat het studenten kennelijk ontbreekt aan kennis van Enabling Conditions: ook wanneer informatie met betrekking tot deze ziektescript-component expliciet voorhanden is, wordt deze door hen niet herkend en geselecteerd. 
De resultaten lieten echter geen enkel significant verschil zien tussen de drie expertiseniveaus. Niet alleen selecteerden proefpersonen op de verschillende expertiseniveaus gemiddeld een ongeveer gelijk aantal informatie-elementen voor de casus-reconstructie, ook op de aard van de geselecteerde informatie bleek geneeskundige ervaring geen enkele invloed te hebben. Ook uit enkele extra manipulaties, zoals het laten wegnemen van niet-cruciale informatie, of het verplicht laten uitbreiden van de selectie met aanvankelijk niet-gekozen informatie, bleek geen enkel effect van expertiseniveau. De algemene conclusie luidde dan ook dat nergens uit blijkt dat bij het reconstrueren van een casus experts meer geneigd zijn Enabling Conditions te selecteren dan niet-experts. Het lijkt er dus op dat deze kennis in principe aanwezig is, ook bij gevorderde studenten.

Deze conclusie wordt nader getoetst in het onderzoek waarover in Hoofdstuk 3 wordt gerapporteerd. Dit onderzoek is in feite een replicatie en uitbreiding van het experiment van Hobus et al. (1989). De replicatie hield in dat in het huidige onderzoek, net als in dat van Hobus et al., aan proefpersonen gevraagd werd om, voor twintig verschillende ziekten, een bijbehorende prototypische patiènt te beschrijven. De uitbreiding was tweeledig. Ten eerste werden twee extra expertisew niveaus toegevoegd, te weten vierdejaars-studenten geneeskunde en huisartsen in opleiding, naast zesdejaars-studenten en ervaren huisartsen. Ten tweede werd weliswaar aan de helft van de proefpersonen gevraagd om een prototypische patiënt te beschrijven met elk van de ziekten, maar de andere helft kreeg de opdracht om het ziektebeeld behorend bij de aandoeningen te beschrijven. Deze laatste conditie was toegevoegd omdat de mogelijkheid bestond dat het expliciete verzoek om een prototypische patiënt te beschrijven, proefpersonen ertoe kan hebben aangezet om Enabling Conditions, die eigenlijk (nog) geen deel uitmaken van hun ziektescript-structuren, toch in hun beschrijvingen op te nemen. De achterliggende gedachte was dat de instructie om het ziektebeeld behorend bij een bepaalde ziekte te beschrijven, in plaats van een prototypische patiënt, proefpersonen in hun verhalen veel minder zou sturen in de richting van Enabling Conditions; immers, de term ziektebeeld werd geacht sterker te verwijzen naar klachten en symptomen, dan naar factoren uit de context van de patiënt.

Op grond van de vroegere resultaten werd verwacht dat ervaren artsen in het algemeen meer Enabling Conditions (zowel absoluut aantal als proportioneel) zouden noemen dan minder ervaren proefpersonen. Ook werd een interactie woorspeld tussen expertiseniveau en type instructie: ervaren artsen zouden in beide condities ongeveer dezelfde beschrijvingen geven, inclusief de relevante Enabling Conditions, terwijl met name proefpersonen op de tussenliggende expertiseniveaus (zesdejaars-studenten, huisartsen in opleiding) een zekere gevoeligheid voor het type instructie zouden vertonen, in die zin dat ze (relatief) meer Enabling Conditions zouden noemen indien hen expliciet gevraagd werd een prototypische patiënt te beschrijven, dan wanneer ze een ziektebeeld zouden moeten beschrijven. Voorts werd verwacht dat het aandeel van biomedische informatie in de beschrijvingen een monotoon negatief verband zou vertonen met expertiseniveau.

In grote lijnen werden deze hypothesen door de feiten gestaafd. Zeker tot het niveau van huisartsen in opleiding nam het aantal en de proportie Enabling Conditions in de beschrijvingen toe naarmate de proefpersonen meer ervaring hadden. Daarna is er geen toename meer; de data wijzen er zelfs op dat ervaren huisartsen, met name proportioneel, wat minder Enabling Conditions noemen dan huisartsen in opleiding. Daarnaast bleek er een groot verschill te bestaan tussen de twee condities: indien de proefpersonen een prototypische patiënt moesten beschrijven, werd een omgekeerd U-vormig verband gevonden tussen expertise- 
niveau en aantal en proportie Enabling Conditions. Werd echter gevraagd naar het ziektebeeld, dan bleek er veeleer sprake van een monotone toename van aantal en proportie Enabling Conditions in de beschrijvingen naarmate de proefpersonen meer ervaring hadden. Deze resultaten ondersteunen het idee dat met toenemend expertiseniveau, Enabling Conditions steeds meer integraal onderdeel gaan uitmaken van ziektescripts, in die zin dat zij ook toegankelijk zijn indien zij niet rechtstreeks, dat wil zeggen niet via de instructie om een prototypische patient te beschrijven, aangesproken worden.

Eveneens in overeenstemming met de voorspellingen bleek het aantal biomedische informatie-eenheden in de beschrijvingen zowel relatief als absoluut af te nemen naarmate het expertiseniveau toenam. Dit effect trad op bij beide instructies. Tenslotte werd ook gevonden dat niet zozeer geneeskundige ervaring in het algemeen, maar met name het daadwerkelijk gezien hebben van patiënten met de in het onderzoek gebruikte ziekten, positief gerelateerd was aan het anantal Enabling Conditions in de beschrijvingen: er bleken correlaties in de orde van .35 tot .50 te bestaan tussen het antal patienten dat de proefpersonen gemiddeld aangaven gezien te hebben met een bepaalde ziekte (gemeten met behulp van een rating-scale), en het gemiddelde aantal Enabling Conditions dat werd genoemd. Voor de overige ziektescript-componenten bleek er vrijwel geen verband te bestaan tussen aantal patientcontacten en hoeveelheid informatie in de beschrijvingen. Dus, het lijkt erop dat met name praktische ervaring met patienten de ontwikkeling van ziektescripts, zeker wat betreft de integratie van Enabling Conditions in de kennisstructuren, bevordert.

De experimenten die in de hoofdstukken 4 en 5 worden beschreven hadden met name tot doel het onderzoeken van de "script"-aspecten van ziektescripts. Vergeleken met de onderzoeken uit hoofdstuk 2 en 3 waren deze studies relatief kwantitatief en fijnkorrelig van aard. In Hoofdstuk 4 wordt een experiment beschreven waarin de invloed van expertiseniveau, typiciteit wan casuistiek en ziektescript component (Enabling Conditions en Consequences) op de snelheid waar"mee casusinformatie gelezen, c.q. verwerkt wordt, nader werd onderzocht Te. vens werd nagegaan in hoeverre deze factoren van invloed zijn op de subjectieve waarschijnlijkheid (kansschatting) dat de in een casus beschreven patiënt daadwerkelijk lijdt aan de kwaal waarvan het ziektescript is geactiveerd. In dit onderzoek kregen zesdejaars-gtudenten geneeskunde en ervaren huisartsen een aantal korte, gecomputeriseerde casus te lezen, waarin steeds een patiẻnt werd beschreven met Enabling Conditions en Consequences die hetzij prototypisch, hetzij aty. pisch waren voor de ziekte waaraan deze patient verondersteld werd te lijden. De proefpersonen kregen de opdracht de casus zo snel en zo grondig mogelijk te lezen, om an het einde te beslissen of de patient inderdaad aan deze ziekte, die was aangekondigd aan het begin van de casus, leed. Deze beslissing moest zowel door middel van een dichotome ja/nee keuze, als door middel van een waarschijnlijkheidsschatting, uitgedrukt als percentage, worden genomen.

De leestijden van afzonderlijke casuselementen werden geregistreerd, en de gemiddelde leestijden van Enabling Conditions, Consequences, en gehele casus werden berekend. Voorspeld werd dat ervaren huisartsen de casusinformatie sneller zouden verwerken dan zesdejaars-studenten, dat prototypische informatie sneller verwerkt zou worden dan atypische, en mogelijk dat de meer ervaren proefpersonen wat gevoeliger zouden zijn voor typiciteit van Enabling Conditions dan de studenten. Ook werd verwacht dat typiciteit van de aangeboden informatie van invloed zou zijn op de kansschattingen, waarbij ervaren artsen met typiciteit van zowel Enabling Conditions als Consequences rekening zouden houden, ter- 
wijl de zesdejaars-studenten hun waarschijnlijkheidsoordelen voornamelijk zouden laten bepalen door typiciteit van Consequences.

Inderdaad bleek dat ervaren proefpersonen casusinformatie sneller verwerken dan studenten, en dat prototypische informatie sneller wordt gelezen dan atypische. Echter, de invloed van ziektescript-component was additief van aard, en vertoonde geen interactie met expertiseniveau: de bijdragen van Enabling Conditions en Consequences aan de leestijden waren ongeveer gelijk voor beide expertiseniveaus. Voor de waarschijnlijkheidsschattingen werd wél een interactie gevonden tussen expertiseniveau en ziektescript-component: ervaren huisartsen blijken wat meer rekening te houden met typiciteit van Enabling Conditions bij het schatten van de kans dat een patient lijdt aan een bepaalde ziekte dan zesdejaars-studenten, alhoewel deze laatsten zeker niet helemaal ongevoelig zijn voor typiciteit van deze ziektescript-component. Deze resultaten ondersteunen de gedachte dat de kennisstructuren van experts (ziekte)script-achtige eigenschappen vertonen, en tevens dat bij de ontwikkeling van deze structuren beide ziektescript-componenten, t. w. Enabling Conditions en Consequences, zijn betrokken.

Het in Hoofdstuk 5 gepresenteerde onderzoek tenslotte is in wezen een replicatie, aanpassing, en uitbreiding van eerder script recognition onderzoek verricht door Yekovich en Walker (Walker \& Yekovich, 1984, 1987; Yekovich \& Walker, 1986) en Graesser en zijn collega's (Graesser, 1981; Graesser, Gordon, \& Sawyer, 1979; Graesser, Woll, Kowalski, \& Smith, 1980; Graesser \& Nakamura, 1982; Smith \& Graesser, 1981). In een herkenningstaak werd de invloed van expertiseniveau, het al of niet letterlijk gepresenteerd zijn van casusinformatie, typiciteit, en zielktescript-component op de nauwkeurigheid van de responsen en de bijbehorende reactietijden onderzocht. De experimentele taak bestond uit twee onderdelen. In een leerfase kregen vierdejaars- en zesdejaars-studenten geneeskunde en ervaren huisartsen gecomputeriseerde casus aangeboden. De diagnose verbonden aan de casus werd van tevoren bekend gemaakt; de enige taak die de proefpersonen moesten uitvoeren was het lezen en begrijpen van de casus. De presentatieduur van alle casuselementen, die de vorm hadden van korte zinnetjes, was van tevoren vastgesteld en voor alle proefpersonen gelijk. Na een korte tussentaak werd de testfase gestart. Gedurende deze testfase kregen de proefpersonen casuselementen aangeboden, waarvan zij moesten vaststellen, zo snel en nauwkeurig mogelijk, of deze letterlijk op het scherm te zien waren geweest gedurende de presentatie van de overeenkomstige casus in de leerfase, of niet. Typiciteit, feitelijke presentatie, en ziektescript-component van de test-elementen werden experimenteel gemanipuleerd. De reactietijd voor elk element werd geregistreerd, evenals de kwaliteit van de response: hit, false alarm, correct rejection, of miss. In navolging van het voornoemde script-onderzoek werd voorspeld dat de proefpersonen atypische casus-elementen beter zouden kunnen herkennen dan prototypische, en dat dit verschil groter zou zijn voor de ervaren artsen dan voor de studenten. Ervaren artsen zouden met name geneigd zijn prototypische, maar niet letterlijk gepresenteerde casusinformatie, ten onrechte te herkennen als wél letterlijk aangeboden. Wat betreft de reactietijden werd verwacht dat deze korter zouden worden met toenemend expertiseniveau, dat op atypische casuselementen sneller gereageerd zou worden dan op prototypische, en dat met name prototypische, niet letterlijk gepresenteerde casusinformatie aanleiding zou geven to lange reactietijden. Daarnaast werd ook een interactie tussen expertiseniveau en typiciteit werwacht, in die zin dat ervaren artsen in het algemeen snellere responsen zouden geven dan gevorderde studenten en onervaren artsen, maar relatief meer moeite zouden hebben met prototypische, niet letterlijk aangeboden informatie. Tenslotte werden de huidige resultaten vergeleken met die van Yekovich 
en Walker (1986), om na te gaan of het door hen gepresenteerde stadia-model van het verwerken van script informatie, ook van toepassing zou zijn op ziektescripts.

Uit de resultaten bleek inderdaad dat prototypische, niet letterlijk aangeboden casuselementen vaak ten onrechte herkend werden, veel vaker dan atypische, niet letterlijk aangeboden elementen. Dit effect was sterker voor ervaren artsen dan voor studenten. Voor alle drie de expertiseniveaus gold tevens dat dit resultat met name het gevolg was van de reacties op Enabling Conditions, en minder van die op Consequences. Daarmaast bleken proefpersonen op alle drie de expertiseniveaus relatief lange reactietijden te vertonen voor niet letterlijk gepresenteerde, prototypische informatie. Dit effect was ook weer voornamelijk toe te schrijven aan de Enabling Conditions. De vergelijking van de huidige resultaten met die van Yekovich en Walker (1986) liet een gemengd beeld zien. De reactietijden voor hits en correct rejections voor ziektescript-informatie vertoonden een patroon overeenkomstig met dat van dit type informatie in klassieke scripts. Echter, waar false alarms in het eerdere onderzoek voornamelijk een gevolg leken van snelle, onterechte, "herkenning", leken ze in het huidige onderzoek eerder het resultaat van een weloverwogen, doch verkeerd uitpakkende beslissing.

Een opvallende bevinding van het onderzoek in Hoofdstuk 5 was dat, in tegenstelling tot eerder onderzoek (bw. Arkes \& Harkness, 1980), de ervaren artsen in de huidige studie niet geneigd waren prototypische informatie die opzettelijk uit de casus was weggelaten, maar in de testfase wel werd aangeboden, ten onrechte te herkennen. Dit in tegenstelling to geparafraseerde informatie, dat wil zeggen testelementen die qua inhoud overeenkwamen met oorspronkelijke casuselementen, maar niet letterlijk hetzelfde waren: deze laatste werden door ervaren artsen wél relatief vaak ten onrechte herkend. Kennelijk wordt de letterlijke bewoording waarin casusinformatie is gesteld snel vergeten, maar zijn artsen niet geneigd voor een ziekte prototypische informatie automatisch af te leiden, wanneer deze in de casus niet vermeld wordt.

Samenvattend kan gesteld worden dat ziektescripts een aantal belangrijke eigenschappen die tot uitdrukking komen in waarneembaar gedrag, zoals verwerkingssnelheid en geheugenprestaties op een herkenningstaak, gemeen hebben met de scripts van Schank en Abelson (1977). Bovendien kan ook geconcludeerd worden dat ziektescripts met toenemende praktijkervaring steeds meer geîntegreerd en geconsolideerd worden. De veronderstelde differentiële roll van Enabling Conditions en Consequences komt echter niet zo goed uit de verf: in een vrije produktie taak worden weliswaar aan expertiseniveau gerelateerde verschillen gevonden in taegankelijkheid als gevolg van type instructie, maar in een ziektescript-herkenningstaak blijken er nauwelijks verschillen te bestaan tussen ervaren huisartsen en studenten wat betreft geheugenprestaties voor beide ziektescript-componenten.

In Hoofdstuk 6 tenslotte worden de implicaties besproken van de huidige onderzoeksresultaten voor de representatie van medisch-diagnostische kennis in de vorm wan ziektescripts, voor de ontwikkeling van deze scripts, en voor de geneeskundige onderwijspraktijk. Betoogd wordt dat ziektescripts een betere verklaring geven voor de onderzoeksresultaten dan andere vormen van kennisrepresentatie, zoals prototypie en mentale modellen. Voorts wordt beargumenteerd dat het ontwikkelingsaspect van de ziektescript-theorie een potentiële verklaring kan vormen voor de verschillen tussen beginners en experts in de geneeskunde, zoals die in het huidlige onderzoek en de voorlopers ervan gevonden zijn. Enkele voorlopige suggesties worden gedaan met betrekking tot de implicaties van de huidige resultaten, en de ziektescript-theorie, voor de praktijk van het geneeskundig onderwijs. Tenslotte worden voorstellen geformuleerd voor vervolgonderzoek. 


\section{REFERENCES}

Abbott, V., Black, J. B., \& Smith, E. E. (1985). The representation of scripts in memory. Journal of Memory and Language, 24, 179-199.

Abelson, R. P. (1981). Psychological status of the script concept. American Psyehologist, $36,715-729$.

Adelson, B. (1981). Problem solving and the development of abstract categries in programming languages. Memory \& Cognition, 9,422-433.

Ahn, W., Brewer, W. F. \& Mooney, R. J. (1992). Schema acquisition from a single example. Journal of Experimental Psychalogy: Learning, Memory, and Cognition, 18, $391-412$.

Allen, S. W., Brooks, L. R., \& Norman, G. R. (1988). Effect of prior examples on rule based diagnostic performance. In Research in Medical Education: 1988. Pro. ceedings of the Twenty-Fifth Annual Conference. Washington, D.C.: Association of American Medical Colleges, 1988.

Allen, S. W., \& Brooks, L. R. (1991). Specializing the operation of an explicit rulle. Journal of Experimental Psychology: General, 120, 3-19.

Anderson, J. R. (1983). The architecture of cognition. Cambridge, MA: Harvard University Press.

Anderson, J. R. (1987). Skill acquisition: Compilation of weak-method problem solutions. Psychological Review, 94, 192-210.

Anderson, J. R. (1993), Rules of the mind. Hillsdale, NJ: Lawrence Erlbaum Associates, Publishers.

Anderson, J. R., \& Thompson, R. (1989). Use of analogy in a production system architec. ture. In: S. Vosniadou, \& A. Ortony (Eds.), Similarity and analogical reasoning. Cambridge, UK: Cambridge University Press.

Anderson, R. C., Reynolds, R. E., Schallert, D. L., \& Goetz, E. T. (1977). Frameworks for comprehending discourse. Journal of Educational Psychology, 75, 271-278.

Anderson, S. J., \& Conway, M. A. (1993). Investigating the structure of autobiographical memories. Journal of Experimental Psychology: Learning, Memory, and CogniLion, 19, 1178-1196.

Anzai, Y. (1991). Learning and use of representations for physics research . In: K. A. Ericsson, \& J. Smith (Eds.), Toward a general theory of expertise: Prospects and limits. Cambridge, MA: Cambridge University Press.

Anzai, X. Simon, H. A. (1979). The theory of learning by doing. Psychological Review, $86,124-140$.

Arkes, H. R., \& Harkness, A. R. (1980). Effect of making a diagnosis on subsequent recognition of symptoms. Journal of Experimental Psychology: Human Learning and Memory, 6, 568-575.

Barrows, H. S., \& Feltovich, P. J. (1987). The clinical reasoning process. Medical Educaion, $21,86-91$.

Barrows, H. S., Feightner, J. W., Neufeld, V. R., Rorman, G. R. (1978). Analysis of the clinical methods of medical students and physictans. (Report No ODH-PR-233 and ODH-DM-226. Hamilton, Ontario, Canada: McMaster University.

Barrows, H. S., Norman, G. R., Neufeld, V. R., Feighther, J. W. (1982). The clinical reasoning of randomly selected physicians in general medical practice. Clinical $d$ Investigative Medicine, 5, 49-55.

Barsalou, L. W., Sewell, D. R. (1985). Contrasting the representation of scripts and categories. Journal of Memory and Language, 24, 646-665.

Bartlett, F. C. (1932/1954). Remembering. A study in experimental and social psychology. Cambridge, UK: The Syndics of the Cambridge University Press.

Bechtel, W., \& Abrahamsen, A. (1991). Connectionism and the mind: An introduction. Oxford, UK: Blackwell.

Bellezza, F. S., \& Bower, G. H. (1981). The representational and processing characteristics of scripts. Bulletin of the Psychonomic Society, 18, 1-4. 
Benbassat, $J$. . B Schiffmann, A. (1976). An approach to teaching the introduction to chincal medicine. Annals of Internal Medicine, $84,477481$.

Biela, A. (1986). Psychological patterms in predicting disjunction and conjunction of clinical symptoms. Acta Psychologica, 61, 183-195.

Bobrow, D. G. \& Norman, D. A. (1975). Some principles of memory schemata. In D. G. Bobrow \& A. M. Collins (Eds.), Representation and understanding: Sulies in cog. nitue science. Now York: Academic Press.

Borak, $J$, \& Veilleux, S. (1982). Errors of intuitive logic among physicians. Social Science and Medicine, 16, 1939-1947.

Bordage, G. (1984). Diagnostic errors: poor reasoning habits or ill-structured knowledge. In H. G. Schmidt, \& M. L. de Volder (Eds.), Tutorials in problem-based learning. New directions in training for the health professions. Assen/Maastricht: Van Gorcum.

Bordage, G., \& Zacks, R. (1984). The structure of medical knowledge in the memories of medical students and general practitioners: Categories and prototypes. Medical Education, $18,406-416$.

Boshuizen, H. P. A. (1989). De ontwikeling van medische expertise: Een cognitiefpsychologische benadering. [The development of medical expertise: a cognitive-psychological approach] Maastricht: Rijiksuniversiteit Limburg. Doctoral thesis.

Boshuizen, H. P. A., \& Schmidt, H. G. (1990). Biomedical knowledge and clinical expertise. Paper presented at the Annual Meeting of the American Educational Research Association. Boston. MA.

Boshuizen, H. P. A., \& Schmidt, H. G. (1992). On the role of biomedical knowledge in clinical reasoning by experts, intermediates and novices. Cognitive Sicience, 16, 153184.

Boshuizen, H. P. A., Schmidt, H. G., \& Coughlin, L. D. (1988). On the application of medical basic-science knowledge in clinical reasoning: implications for structural knowledge differences between experts and novices. In: Program of the Tenth Annual Conference of the Cognitive Science Society. Montreal, Quebec, Canada. (pp. 517-523).

Bower, G. H., Black, J. B., \& Tumer, T. J. (1979). Scripts in memory for text. Cognitive Psychology, 11, 177-220.

Bransford, J. D., \& Franks, J. J. (1972). The abstraction of linguistic ideas: a review. Cognition, $1,211-249$.

Bransford, J. D., \& Johnson, M. K. (1972), Contextual prerequisites for understanding: Some investigations of comprehension and recall. Journal of Verbal Learning and Verbal Behawior, 11,717-726.

Bransford, J. D., Franks, J. J., Vye, N. J., Sherwood, R. D. (1989). New approaches to instruction: because wisdom can't be told. In: S. Vosniadou, \& A. Ortony (Eds.), Similarity and analogical reasoning. (pp. 470-497). Cambridge, UK: Cambridge Uniwersity Press.

Brewer, W. F. (1986). What is autobiographical memory? In: D. C. Rubin (Ed.), Autobiographical Memory. Cambridge: Cambridge University Press.

Brewer, W. F. (1987). Schemas versus mental models in human memory. In: P. Morris (Ed.), Modelling cognition. (pp. 187-197). New York: John Wiley \& Sons Ltd.

Brooks, L. R. (1987). Decentralized control of categorization: the role of prior processing episodes. In: U. Neisser (Ed), Concepts and conceptual development. Ecological and intellectual factors in categorization. Cambridge: University Press.

Brooks, L. R., Norman, G. R., \& Allen, S. W. (1991). Role of specific simillarity in a medical diagnostic task. Journal of Experimental Psychology: General, 120, 278-287.

Bruner, J. S., Goodnow, J., \& Austin, G. (1956). A study of thinking. New York: Wiley.

Camerer, C. F., Johnson, E. J. (1991). The process-performance paradox in expert judgment: How can experts know so much and predict so badly. In: $\mathbb{K}$. A. Ericsson, \& I. Smith (Eds.), Toward a general theory of expertise: Prospects and limits. Cambridge, MA: Cambridge University Press. 
Cantor, N., Smith, E. E., French, R., \& Mezrich, J. (1980). Psychiatric diagnoses as prototype categorization. Journal of Abnormal Psychology, 89, 181-193.

Carey, S. (1985). Conceptual change in childhood. Cambridge, MA: The MIT Press.

Carlson, R. A. \& Dulany, D. E. (1988). Diagnostic reasoming with circumstantial evidence. Cognitive Psychology, 20, 463-492.

Carlson, R. A., Khoo, B. H., Yaure, R. G., \& Schneider, W. (1990). Acquisition of a problem-solving skill: Levels of organization and use of working memory. fournal of Experimental Psychology: General, 119, 193-214.

Carmody, D. P., Kundel, H. L., \& Toto, L. C. (1984). Comparison scans while rasading chest images: taught, but not practiced. Investigative Radiology, 19,462-466.

Charness, N. (1979). Components of skill in bridge. Canadian Journal of Psychology, 33, $1-16$

Charness, N. (1989). Expertise in chess and bridge. In: D. Klahr, \& K. Kotovsky (Eds.), Complex information processing: The impact of Herbert A. Simom. (pp. 183-208). Hillsdale, NJ: Lawrence Erlbaum Associates.

Chase, W. G., E Ericsson, K. A. (1981). Skilled memory. In II. R. Anderson (Ed.), Cognitive skills and their acquisition (pp 141-189). Hillsdale, NJ: Lawrence Enrbaum Associates, Publishers.

Chase, W. G., \& Simon, H. A. (1973). Perception in chess. Cognitive Psychology, 4, 55-81.

Chi, M. T. H., Bassok, M. Lewis, M. W., Reimann, P., \& Glaser, R. (1989). Self-explanations: How students study and use examples in learning to solve problems. Cognitive Science, $13,145-182$.

Chi, M. T. H. Feltovich, P. J., \& Glaser, R. (1981). Categorization and representation of physics problems by experts and novices. Cognitive Science, 5, 121-152.

Christensen-Szalanski, J. J. \& Bushyhead, J. B. (1981). Physician's use of probabilistic information in a real clinical setting. Journal of Experimental Psychology: Human Perception and Performance, 7, 928-935.

Clancey, W. J. (1983). The epistemology of a rule-based expert system - a framework for explanation. Artificial Intelligence, 20, 215-251.

Clancey, W. J. (1984). Methodology for building an intelligent tutoring system. In: W. Kintsch, J. R. Miller, \& P. G. Polson (Eds.), Method and tactics in cognitive science. (pp. 51-83). Hillsdale, New Jersey: Lawrence Erlbaum Associates, Publishers.

Clement, J. (1991). Nonformal reasoning in experts and in science students. In: J. F. Voss, D. N. Perkins, \& J. W. Segal (Eds.), Informal reasoning and education. (pp. 345-362). Hillsdale, New Jersey: Lawrence Erlbaum Associates, Publishers.

Coughlin, I. D., \& Patel, V. J. (1987). Processing of critical information by doctors and medical students. Journal of Medical Education, $62,818-828$.

Cutler, P. (1979). Problem solving in chinical medicine: From dato to diagnosis. Baltimore: The Williams \& Wilkins Company.

De Groot, A. D. (1946/1978). Thought and choice in chess. Den Haag, The Netherlands: Mouton.

Dreyfus, H. L. , \& Dreyfus, S. E. (1984). Mindless machines. Computers don't think like experts, and never will. The Sciences, November-issue 1984, 18-22.

Dreyfus, H. L., \& Dreyfus, S. E. (1986a). Mind over machine. The pouer of human inuition and expertise in the era of the computer. Oxford, U.K. Basil Blackwell Ltd.

Dreyfus, H. L., \& Dreyfus, S. E. (1986b). Why skills camnot be represented by rules. In $\mathrm{N}$. E. Starkey, (Ed.), Advances in cognitive science. Volume 1. Chichester, GB: Ellis Horwood Ltd.

Ehrlich, K., \& Soloway, E. (1984). An empirical investigation of the tacit plan knowledge in programming. In: J. C. Thomas, \& M. L. Schneider (Eds.), Human factors in computer systems. (pp. 113-133). Norwood, New Jersey: Ablex Publishing Corporation.

Einhorn, H. J. (1974). Expert judgment: Some necessary conditions and an example. Journal of Applied Psychology, 59,562-571.

Elio, R., \& Scharf, P. B. (1990). Madeling novice-to-expert shifts in problem-8olving strategy and knowledge organization. Cognitive Science, 14, 579-639. 
Elahout, J. J. (1983). Een beginner is méer dan iemand die het nog niet kan. There is more to novice than not yet being able to perform correctly]. In: P. J. D. Drenth, W. Koops, J. F. Orlebeke, \& R. J. Takens (Eds.), Psychologie in Nederland enkele ontwik helingen in 1982. (pp. 177-184). Amsterdam/Lisse: N.I.P./Swets \& Zeitlinger.

Elstein, A. S. Shulman, L. S., Sprafka, S. A. (1978). Medical problem solving. An ana. lysis of clinical reasoning. Cambridge, MA/London, UK: Harvard University Press.

Elstein, A. S., Shulman, L. S., \& Sprafka, S. A. (1990). Medical problem solving: A tenyear retrospective. Evaluation \& The Health Professions, 13,5-36.

Ericsson, K. A. \& Smith, J. (1991). Prospects and limits of the empirical study of expertise: an introduction. In: K. A. Ericsson \& J. Smith (Eds.), Toward a general theory of expertise: Prospects and limits. Cambridge, MA: Cambridge University Press.

Ericsson, K. A., Krampe, R. T, \& Tesch-Römer, C. (1993). The role of deliberate practice in the acquisition of expert performance. Psychological Review, $100(3), 363-406$.

Evans, D. A. (1989). Issues of cognitive science in medicine. In: D. A. Evans, \& V. L. Patel (Eds.), Cognitive science in medicine: biomedical modeling. (pp. 1-19). Cambridge, MA: The MTT Press.

Evans, J. S. B. (1989). Bias in human reasoning: Causes and consequences. Hove, U.K. Law rence Erlbaum Associates, Publishers.

Feigenbaum, E. A., Feldman, J. (1963). Computers and thought. New York: McGrawHill.

Feldman, J. A. (1989). A connectionist model of visual memory. In: G. E. Hinton, \& J. A. Anderson (Eds.), Parallel models of associative memory. Updated edition. (pp. 6597). Hillsdale, New Jersey: Lawrence Errbaum Associates.

Feltovich, P.J., \& Barrows, H. S. (1984). Issues of generality in medical problem solving. In H. G. Schmidt, \& M. L. De Volder, (Eds.), Tutorials in problem-based learning. New directions in training for the health professions. (pp. 128-142). Assen/Maastricht: Van Gorcum.

Feltovich, P. J., \& Coulson, R. L. (1992). Conceptual knowledge foundations for naval medical training: A scheme for directed curricular planning and instructional design. Final Report: Navy Manpower Personnel, and Training R\&D Program.

Feltovich, P. J., Coulson, R. L., Spiro, R. J.s \& Dawson-Saunders, B. K. (1992). Knowledge application and transfer for complex tasks in ill-structured domains: implications for instruction and testing in biomedicine. In: D. Evans, \& V. L. Patel (Eds.), Advanced models of cognition for medical training and practice. New York: Springer Verlag.

Feltovich, P. J., Johnson, P. E., Moller, J. H., \& Swanson, D. B. (1984). LCS: The role and development of medical knowledge in diagnostic expertise. In: W. J. Clancey, \& $\mathbb{E}$. H. Shortliffe (Eds.), Readings in medical artificial inteligence: the first decade (pp. 275-319). Reading, MA: Addison-Wesley Publishing Company.

Fodor, J. A. (1983). The modularity of mind. Cambridge, MA: The MIT Press.

Forsythe, G. B. \& Barber, H. F. (1992). Military strategic thinking: Expert-novice differences in the structure and content of cognitiwe representations. Paper presented at the Annual Meeting of the American Educational Research Association, San Francisco, CA, April 1992.

Gale, J., \& Marsden, P. (1983). Medical diagnosis. Oxford: Oxford University Press.

Garland, L. H. (1959). Studies on the accuracy of diagnostic procedures. American Journal of Roentgenology, 82, $25-38$.

Garrod, S., Sanford, A. (1983). Topic dependent effects in language processing. In G. B. Flores D'Arcais, \& R. J. Jarvella (Eds.), The process of language understanding. (pp. 271-296). Chichester: John Wiley \& Sons.

Genero, N., \& Cantor, N. (1987). Exemplar prototypes and clinical diagnosis: Toward a cognitive economy. Journal of Social and Clinical Psychology, 5, 59-78.

Gentner, D. \& Stevens, A. I. (Eds.) (1983). Mental models. Hillsdale, NJ: Lawrence Erlbaum Associates, Publishers. 
Gerritsma, J. G. M., \& Smal, J. A. (1982). De werkwize van huisaris en internist. Eien vergelijkend onderzoek met behulp van een interactieve patientensimulatie. Utrecht: Wetenschappelijke Uitgeverij Bunge.

Gick, M. L., \& Holyoak, K. J. (1983). Schema induction and analogical transfor. Cognitive Psychology, 15, 1-38.

Gilhooly, K. J. (1990). Cognitive psychology and medical diagnosis. Applied Cognitive Psychology, 4, 261-272.

Gillhooly, K. J., \& Green, A. J. K. (1988). The use of memory by experts and novices. In: A. M. Colley, \& J. R. Beech (Eds.), Cognition and action in skilled behaviour. Series Eds: G. Stelmach, \&. A. Vroon (Advances in psychology. Vol. 55). (pp. 379.395). Amsterdam: Elsevier Science Publishers B. V.

Glaser, R. (1986). On the nature of expertise. In: F. Klix, \& H. Hagendorf (Eds .), Human memory and cognitive capabilities: Mechanisms and performances. 2 (pp. 915-928). Amsterdam: Elsevier Science Publishers B.V.

Glaser, R. (1990). Expert knowledge and the thinking process. Chemtech, 20, 394-397.

Godden, D. R. \& Baddeley, A. D. (1975). Context-dependent memory in two natural environments: On land and underwater. British Journal of Psychology, 66, 325 331.

Graesser, A. C. (1981). Prose comprehension beyond the word. New York: Springer Verlag.

Graesser, A. C., \& Nakamura, G. V. (1982). The impact of a schema on comprehension and memory. In: G. H. Bower (Ed.), The psychology of learning and motivation. Advances in research and theory. Volume 16. (pp. 59-109). New York: Academic Press.

Graesser, A. C., Gordon, S. E., \& Sawryer, d. D. (1979). Recognition memory for typical and atypical actions in scripted activities: Tests of a script pointer + tag hypothesis. Journal of Verbal Learning and Verbal Behavior, 18, 319-332.

Graesser, A. C., Woll, S. B., Kowalski, D. J., \& Smith, D. A. (1980). Memory for typical and atypical actions in scripted activities. Journal of Experimental Psychology: Human Learning and Memory, 6, 503-515.

Groen, G. J., \& Patel, V. L. (1985). Medical problem solving and cognitive psychology: some questionable assumptions. Medical Education, 19, 95-100.

Hamm, R. M. (1988). Clinical intuition and clinical analysis: expertise and the cognitive continuum. In: J. Dowie, \& A. Elstein (Eds.), Professional judgment. A reader in clinical decision making. (pp. 78-105). Cambridge, U.K.: Cambridge University Press.

Hassebrock, F., \& Prietula, M. J. (1990). Autobiographical memory in medical problem solving. Paper presented at the Annual meeting of the American Educational Research Assaciation. Boston, MA.

Hassebrock, F., Prietula, M. J. (1992). A protocol-based coding scheme for the analysis of medical reasoning. International Journal of Man-Machine Studies, 37, 613-652.

Hobug, P. P. M. (1994). Expertise van huisartsen. Praktijkervaring, hennis en diagnos: tische hypothegevorming. [Family physicians' expertise: Practical experience, knowledge, and formation of diagnostic hypotheses]. Maastricht, The Netherlands: Doctoral dissertation.

Hobus, P. P. M., Boshuizen, H. P. A., \& Schmidt, H. G. (1989). Mental representation of prototypical patients: expert-novice differences. Paper presented at the First European Congress of Psychology, July 2-7, 1989, Amsterdam, The Netherlandis.

Hobus, P. P. M., Boshuizen, H. P. A., \& Schmidt, H.. G. (1990). Expert-novice differences in the role of contextual factors in early medical diagnosis. Paper presented at the Annual Meeting of the American Educational Research Association. Bioston, MA.

Hobus, P. P. M., Hofstra, M. L., Boshuizen, H. P. A., \& Schmidt, H. G. (1988). De context van de klacht als diagnosticum [The context of the complaint as diagnostic tooll. Huisarts en Wetenschap, 31, 261-267.

Hobus, P. P. M., Schmidt, H. G., Bashuizen, H. P. A., \& Patel, V. L. (1987). Contextual factors in the activation of first diagnostic hypotheses: expert-novice differences. Medical Education, $21_{n} 471-476$. 
Hodgkin, K (1984). Vroege diagnostiek in de eerste lun. Lochem/Poperinge: Uitgeversmatschappij "de Tijdstroom".

Hofstadter, D. R. (1985). Metamagical themas: Questing for the essence of mind and matter. New York: Basic Baoks.

Hofistra, M. L., Hobus, P. P. M., Boshuizen, H. P. A., Schmidt, H. (1988). Diagnostiek buj huisartsen: het belang van gegevens wit de context van de patiènt. [Family physicians" diagnoses: The importance of patient contextual informationl. Maastricht, Rijksuniversiteit Limburg: Onderzoek van Onderwijs.

Holyoak, K. J. (1991). Symbolic connectionism: toward third-generation theories of expertise. In: K. A. Ericsson, \& J. Smith (Eds.), Toward a general theory of expertise. Prospects and limits. (pp. 301-335). Cambridge, U.K: Cambridge University Press.

Holyoak, K. J., \& Thagard, P. R. (1989). Analogical reasoning as a mechanism in knowledge acquisition: a developmental perspective. In: S. Vosniadou, \& A. Ortony (Eds.), Similarity and analogical reasoning. (pp. 242-266). Cambridge, UK: Cambridge University Press.

Johnson, P. E., Durán, A. S., Hassebrock, F., Moller, J., Prietula, M. (1981). Expertise and error in diagnostic reasoning. Cognitive Science, 5, 235-283.

Johnson, S. D. (1988). Cognitive analysis of expert and novice troubleshooting performance. Performance Improvement Quarterly, 1, 38-54.

Johnson-Laird, P. N. (1983). Mental models: Towards a cognitive science of language, inference, and consciousness. Cambridge, UK: Cambridge University Press.

Jones, J. G., Cason, G. J., Cason, C. (1986). The acquisition of cognitive knowledge through clinic experiences. Medical Education, 20, 10-12.

Kahneman, D., Slovic, P., \& Tversky, A. (1982). Judgment under uncertainty: Heuristics and biases. Cambridge, UK: Cambridge University Press.

Kaufman, D. R., \& Patel, V. P. (1988). The nature of expertise in the clinical interview: Interactive medical problem solving. In: Program of the Tenth Annual Conference of the Cognitive Science Society: Montreal, Quebec, Canada. (pp. 461-467).

Klahr, D. (1984). Transition processes in quantitative development. In: R. J. Sternberg (Ed.), Mechanisms of cognitive development. New York: Freeman.

Klayman, $J$, \& Brown, K. (1993). Debias the environment instead of the judge: An alternative approach to reducing error in diagnostic (and other) judgment. Cognition, $49,97-122$.

Koedinger, K. R., \& Anderson, J. R. (1990). Abstract planning and perceptual chunks: elements of expertise in geometry. Cognitive Science, 14, 511-550.

Kolodner, J. L., \& Riesbeck, C. K. (1986). Introduction. In J. L. Kolodner, \& C. K. Riesbeck (Eds,), Experience, memory and reasoning. Hillsdale, New Jersey: Lawrence Errbaum Associates.

Kuipers, B. \& Kassirer, J. P. (1984). Causal reasoning in medicine; Analysis of a protocol. Cognitive Science, $8,363-385$.

Kuipers, B., Moskowitz, A. J., \& Kassirer, J. P. (1988). Clinical decisions under uncertainty: Representation and structure. Cogmitive Science, 12, 177-210.

Labov, W. (1973). The bounderies of words and their meanings. In C.-J. N. Bailey \& R. W. Shuy (Eds), New ways of analyzing variations in English. Washington, DC: Georgetown University Press.

Lakoff, $\mathrm{G}_{n}$ (1987). Women, fine, and dangerous things. What categories reveal about the mind. Chicago: The University of Chicago Press.

Lancaster, J., Kolodner, J. (1988). Varieties of learning from problem solving experience. In Program of the Tenth Annual Conference of the Cognitive Science Shociety. Montreal, Canada.

Lesgold, A. M. (1984). Acquiring expertise. In: ¿. $\mathbb{R}$. Anderson, \& S. M. Kosslyn (Eds.), Tutorials in learning and memory. Essays in honor of Gordon Bower. (pp. 31-60). San Francisco: W. H. Freeman and Company.

Lesgold, A. M. (1989). Context-specific requirements for models of expertise. In: D. A. Evans, \& V. L. Patel (Eds.), Cognitive science in medicine: Biomedical modeling. (pp. 375-400). Cambridge, MA: The MIT Press. 
Maier, N. R. F. (1930). Reasoning in humans I. On direction. Journol of Comparitive psychology, 10, 115-143.

Maier, N. R. F. (1931). Reasoning in humans II. The solution of a problem and its appearance in consciousness. Journal of Comporitive psychology, 12, 181-194.

Maki, R. (1990). Memory for script actions: Effecte of relevance and detail expectancy. Memory \& Cognition, 18, 5-14.

Mannes, S. M., \& Doane, S. M. (1991). A hybrid model of script generation: Or getting the best from both worlds. Connection Science, 3,61-87.

McClelland, J. L., \& Rumellart, D. E. (1986). Parallel distributed processing: Explo. rations in the microstructure of cognition. Vols. $1 \& 2$. Cambridge, MA: MIT Press.

McKoon, G. \& Ratcliff, R. (1989). Inferences about contextually dehned categories. Jour. nal of Experimental Psychology: Learning, Memory, and Cogmition, 15, 1134-1146.

Medin, D. L. (1989). Concepts and conceptual structure. Anerican Psychologist, 44, 14691481.

Medin, D., \& Ortony, A. (1989). Psychological essentialsm. In: S. Vosniadou, \& A. Ortony (Eds.), Similarity and analogical reasoning. (pp. 179-195). Cambridge, UK: Cambridge University Press.

Miller, G. A. (1956). The magical number seven, plus or minus two: Some limits on our capacity for processing information. Psychological Review, 63, 81-97.

Miller, G. A., Galanter, E., \& Pribram, K. H. (1960). Plans and the structure of behavior. New York: Holt, Rinehart and Winston.

Minsky, M. A. (1975). A framework for representing knowledge. In: P. H. Winston (Ed.), The psychology of computer wision. New York: McGraw Hill.

Mitchell, T. M., Keller, R. M., Kedar-Cabelli, S. T. (1986). Explanation-based generalization: a unifying view. Machine Learning, $1,47-80$.

Murphy, G. L. \& Brownell, H. H. (1985). Category differentiation in object recognition: Typicality constraints on the basic category advantage. Joumal of Experimental Psychology: Learning, Memory, and Cognition, 11, 70-84,

Murphy, G. L., Medin, D. L. (1985). The role of theories in conceptual coherence. Psychological Review, 92, 289-316.

Murphy, G. L., \& Wright, J. C. (1984). Changes in conceptual structure with expertise: differences between real-world experts and novices. Journal of Experimentel Psychology: Learring, Memory and Cognition, 10, 144-155.

Nakamura, G. V., \& Graesser, A. C. (1985). Memory for script-typical and script-atypical actions: A reaction time study. Bulletin of the Psychonomic Society, 23, 384-386.

Nakamura, G. V., Graesser, A. C., Zimmerman, J. A., \& Riha, J. (1985). Script proces. sing in a natural situation. Memory \& Cognition, 13, 140-144.

Neufeld, W. R. Norman, G. R., Feightner, J. W., Barrows, H. S. (1981). Clinical problem solving by medical students: A cross-sectional and longitudinal analysis. Medical Education, 15, 315-322.

Newell, A. (1990). Unified theories of cognition. Cambridge, MA: Harward Univergity Press.

Newell, A., Shaw, J. C \& Simon, H. A. (1958). Elements of a theory of human problem solving. Psychological Review, 65, 151-166.

Newell, A., \& Simon, H. A. (1972). Human problem solving. Englewood Cliff, Ny: Prentice-Hall, Inc.

Nisbett, R. E., \& Ross, L. (1980). Human inference: Strategies and shortcomings of social judgment. Englewood Cliffs, NJ: Prentice-Hall, Inc.

Norman, G. R. (1988). Problem-solving skills, solving problems and problem-based learning. Medical Education, 22, 279-286.

Norman, G. R., Brooks, L. R., \& Allen, S. W. (1989). Recall by expert medical practition* ers and novices as a record of processing attention. Joumal of Experimental Psy. chology: Learning, Memory, Cognition, 15, 1166-1174.

Norman, G. R., Brooks, L. R., Allen, S. W. \& Rosenthal, D. (1988). Retrieval factors in medical expertise: improvement independent of stable knowledge. In Proceedings 
of the Tenth Annual Conference of the Cognitive Science Society. Montreal, Quebec, Canada.

Norman, G. R., Rosenthal, D., Brooks, L. R., \& Muzzin, L. J. (1989). The development of expertise in dermatology. Archives of Dermatology, 125, 1068-1068.

Norman, G. R., Trott, A. L., \& Brooks, L. R. (1992). Cognitive difficulties in clinical reasoning related to postgradaate training. Paper presented at the Annual Meeting of the American Educational Research Association. San Francisco, CA: April 20-24,

Novick, L. R. (1988). Analogical tramsfer, problem similarity and expertise. Joumal of Experimental Psychology: Learning, Memory and Cognition, 14, 510-520.

Nurcombe, B, \& Fitzhenry-Coor, I. (1982). How do psychiatrists think? Clinical rea soning in the psychiatric interview: A research and education project. Australian and New Zealand Journal of Psychiatry, 16, 13-24.

Olson, J. R. \& Biolsi, K. J. (1991). Techniques for representing expert knowledge. In $\mathrm{K}$. A. Ericsson, \& J Smith (Eds.), Toward a general theory of expertise." Prospects and limits. Cambridge, MA: Cambridge University Press.

Parks, T. E. (1966). Signal detectability theory of recognition memory performance. Psychological Review, 73, 44-58.

Patel, V. J., \& Coughlin, L. D. (1985). Cognitive science and the assessment of clinical competence. In: Proceedings of the International Conference on Newer Developments in Assessing Clinical Competence. Ottawa, Canada.

Patel, V.J., \& Federiksen, C. L. (1984). Cognitive processes in comprehension and knowledge acquisition by medical students and physicians. In $\mathbf{H}$. G. Schmidt, \& M. L. de Volder (Eds... Tutorials in problem-based learning: New directions in training for the health professions. Assen/Maastricht: Van Gorcum.

Patel, V.J., \& Groen, G. J. (1986a). Knowledge based solution strategies in medical reasoning. Cognitive Science, $10,91-116$.

Patel, V. J., \& Groen, G. J. (1986b). The role of mental models in causal explanations by medical students. Paper presented at the AERA Conference, San Francisco, 1986. San Francisco.

Patel, V. L., Evans, D. A., \& Groen, G. J. (1989). Biomedical knowledge and clinical reasoning. In: D. A. Evans, \& V. L. Patel (Eds.), Cognitive science in medicine: Biomedical modeling. (pp. 53-112). Cambridge, MA: The MIT Press.

Patel, V. L., Groen, G. J., \& Arocha. (1990). Medical expertise as a function of task difficulty. Memory \& Cognition, 18, 394-406.

Patel, V. L., Groen, G. J., \& Frederiksen, C. H. (1986). Differences between medical students and doctors in memory for clinical cases. Medical Education, 20,3-9.

Paycha, $\mathbb{F}$. (1955). Critique scientifique des démarches intellectuelles du diagnostic. La. Presse Medicale, 63, 1753-1756.

Piaget, J. (1929). The child's conception of the world. New York: Harcourt Brace.

Polanyi, M. (1958/1973). Personal knowledge. London: Routledge \& Kegan Paul.

Prince, M. (1921). The Uncanscious. The fundamentals of human personality normal and abnormal. New York: The MacMillan Company. (Second Edition Revised)

Read, S. J. (1983). Once is enough: Causal reasoning from a single instance. Journal of Personality and Social Psychology, 45, 323-334.

Reber, A. S. (1989). Implicit learning and tacit knowledge. Journal of Experimental Psychology: General, 118, 219-235.

Reiser, B. J., Black, J. B., \& Abelson, R. P. (1985). Knowledge structures in the organisation and retrieval of autobiographical memories. Cognitive Psychology, 17, 89-137.

Rose, F. (1984). Into the heart of the mind. An American quest for artificial intelligence. New York: Harper \& Row Publishers, Inc.

Rosch, $\mathbb{E}_{*}, \mathrm{H}$. (1975). Cognitive representations of semantic categories. Journal of Experi.mental Psychology: General, 104, 192-233.

Rosch, E. H. (1978). Principles of categorization. In E. Rosch \& B. B. Lloyd (Eds.), Cognition and categorization. Hillsdale, NJ: Lawrence Erlbaum Associates, Publishers. 
Ross, B. H. (1987). This is like that: The use of earlier problems and the separation of similarity effects. Journal of Experimental Psychology: Leanning, Menory, and Cognition, 13,629-639.

Ross, B. H. Perkins, S. J., \& Tenpenny, P. L. (1990). Reminding-based category learning. Cognitive Psychology, 22, 460-492.

Rumelhart, D. E., \& Norman, D. A. (1978). Accretion, tuming and restructuring: Three mades of learning. In: R. Klatzky, \& J. W. Cotton (Eds.), Semantic factors in cognition. (pp. 37-60). Hillsdale, NJ: Lawrence Erlbaum Associates, Publishers.

Rumelhart, D. E. \& Norman, D. A. (1981). Analogical processes in learning. In: J. R. Anderson (Ed.), Cognitive skills and their acquisition. Hillsdale, New Jersey: Lawrence Erlbaum Associates.

Rumelhart, D. E., \& Norman, D. A. (1985). Representation of knowledge. In A. M. Aitkenhead \& J. M. Slack, (Eds.), Issues in cognitive modeling. (pp. 15-62). Hillsdale, $\mathrm{N}_{\mathrm{J}}$ : Lawrence Erlbaum Associates, Publishers.

Salthouse, T. A. (1991). Expertise as the circumvention of human processing limitations: In: K. A. Ericsson, \& J. Smith (Eds.), Toward a general theory of expertise: Prospects and limits. Cambridge, MA: Cambridge University Press.

Sanford, A. J., Garrod, S. C. (1981). Understanding written language. Chichester: John Wiley \& Sons.

Scardamalia, M., Bereiter, C. (1991). Literate expertise. In: K. A. Ericsson, \& J. Smith (Eds.), Toward a general theory of expertise: Prospects and limits. Cambridge, MA: Cambridge University Press.

Schaafstal, A. M. (1991). Diagnostic skill in process operation: A comparison between experts and novices. Groningen, The Netherlands: Rijksuniwersiteit Groningen, Academic Thesis.

Schacter, D. L. (1982). Stranger behind the engram. Theories of memory and the psychology of science. Hillsdale, NJ: Lawrence Erlbaum Associates, Publishers.

Schank, R. C. (1985). Reminding and memory organization. In A. M. Aitkenhead, \&. M. Slack (Eds.), Issues in cognitive modeling. Hillsdale, NJ: Lawrence Erlbaum Associates.

Schank, R. C., \& Abelson, R. P. (1977). Scripts, plans, goals and understanding. An inquiry into human knowledge structures. Hillsdale, New Jersey: Lawrence Er.baum Associates.

Schmidt, H. G., \& Boshuizen, H. P. A. (1992). Encapsulation of biomedical knowledge. In: A. E. Evans, \& V. L. Patel (Eds.), Advanced models of cognition for medical training and practice. New York, NY: Springer Verlag.

Schmidt, H. G., \& Boshuizen, H. P. A. (1993a). On acquiring expertise in medicine. Educational Psychology Review, 5, 205-221.

Schmidt, H. G. \& Boshuizen, H. P. A. (1993b). On the origin of intermediate effects in. clinical case recall. Memory \& Cognition, 21, 338-351.

Schmidt, H. G., \& Norman, G. R. (1989). A stage theory on the development of expertise in medicine. Paper presented at the Annual Meeting of the American Educational Research Association. San Francisco, CA.

Schmidt, H. G., Boshuizen, H. P. A., \& Hobus, P. P. M. (1988). Transitory stages in the development of medical expertise: the "intermediate effect" in clinical case representation studies. In: Program of the Tenth Annual Conference of the Cognitive Science Society. Montreal, Canada. (pp. 139-145).

Schmidt, H. G., Boshuizen, H. P. A. \& Norman, G. R. (1992). Reflections on the nature of expertise in medicine. In: E. Keravnou (Ed.), Deep models for medical knowledge engineering (pp. 231-248). North Holland: Elsevier Science Publishers B. V..

Schmidt, H. G., Norman, G. R. \& Boshuizen, H. P. A. (1990). A cognitive perspective on medical expertise: theory and implications. Academic Medicine, 65, 611-621.

Schraagen, J. M. (1993). How experts solve a novel problem in experimental design. Cognitive Science, 17, 285-309.

Schwartz, S., \& Griffin, T. (1986). Medical thinking. The psychology of medical judgment and decision making. New York: Springer-Verlag. 
Schyns, P. G. (1991). A modular neural network model of concept acquisition. Cognitive Science, $15,461.508$.

Selw, O. (1922). Zur psychologie des produktiven Denkens und des Irrtums. Bonn, Germany: Friedrich Cohen.

Sharkey, N. \& Mitchell, D. C. (1985). Word recognition in a functional context: The use of scripts in reading. Joumal of Memory and Language, 24, 253-270.

Siegel, S. (1956). Nonparametric statistics for the behavioral sciences. New York: MeGraw Hill.

Sloboda, J. (1991). Musical expertise. In: K. A. Ericsson, \& J. Smith (Eds.), Toward a general theory of expertise: Prospects and limits. Cambridge, MA: Cambridge University Press.

Smith, D. A., \& Graesser, A. C. (1981). Memory for actions in scripted activities as a function of typicality, retention interval, and retrieval task. Memory \& Cognition, 9 , $550-559$.

Smith, E. E., \& Medin, D. L. (1981). Categories and concepts. Cambridge, Mass.: Harvard University Press.

Smith, E. E., Langston, C., \& Nisbett, R. E. (1992). The case for rules in reasoning. Cognitive Science, $16,1-40$.

Snoek, J. W. (1989). Het denken van de neuroloog. [Thinking in neurolagy] Groningen, The Netherlands: Academic Thesis.

Spiro, R. J., Feltovich, P. J., Coulson, R. L., \& Anderson, D. K. (1989). Multiple analogies for complex concepts: Antidotes for analogy-induced misconception in adwanced knowledge acquisition. In: S. Vosniadou, \& A. Ortony (Eds.), Similarity and analogical reasoning. (pp. 498-531). Cambridge, UK: Cambridge University Press.

Stermberg, R. J. (1994). The role of tacit knowledge in the professions. Invited address. held at the Annual Meeting of the American Educational Research Association, New Orleans, LA, April 4-8.

Sternberg, R. J., \& Caruso, D. R. (1985). Practical modes of knowing. In: E. Eisner, \& K. J. Rehage (Eds.), Learning and teaching the ways of knowing. Eighty-fourth yearbook of the National Society for the Study of Education. Part II. (pp. 133-158). Chicago, IL: The University of Chicago Press.

Swanson, D. B., Feltowich, P. J., \& Johnson, P. E. (1977). Psychological analysis of physician expertise: Implications for design of decision support systems. In D. B. Shires, \& H. Wolff, Medinfo 77. Proceedings of the Second World Conference on Medical Informatics. Toronto, Canada. (pp. 161-164). Amsterdam/New York: North Holland Publishing Company.

Swanson, D. B., Feltovich, P. J., Johnson, P. E. (1977). Psychological analysis of physician expertise: implications for design of decision support systems. In: D. B. Shires, \& H. Wolff, MEDINFO 77. Proceedings of the Second World Conference on Medical Informatics. Toronto, Canada. (pp. 161-164).

Tanaka, J. W. \& Taylor, M. (1991). Object categories and expertise: Is the basic level in the eye of the beholder? Cognitive Psychology, 23, 457-482.

Tanner, C. A., Padrick, K. P., Westfall, U. E., \& Putziex, D. J. (1987). Diagnostic reasoning strategies of nurses and nursing students. Nursing Research, 36, 358-363.

Tubbs, R. M. (1992). The effect of experience on the auditor's organization and amount of knowledge. The Accounting Review, 67,783-801.

Turner, R. M. (1988). Organizing and. using schematic knowledge for medical diagnosis. In: J. L. Kolodner, Case-based reasoning: Proceedings of a workshop on case-based reasoning. Clearwater Beach, Florida. (pp. 435-446).

Vaatstra, H. $\mathbb{F}$., Boshuizen, H. P. A., \& Schmidt, H. G. (1993). Expertise in auditing: Case representation differences between economy students, novice-auditors, and experts. Paper presented at the Annual Meeting of the American Educational Research Association, Atlanta, GA, April 12-16.

Van de Wiel, M. W., Boshuizen, H. P. A., \& Schmidt, H. G. (1993). A failure to reproduce the intermediate effect in clinical case recall. Paper presented at the Annual Meeting of the American Educational Research Assaciation, Atlanta, GA, April 12-16. 
Van Dijk, T. A. \& Kintsch, W. (1983). Strategies of discourse comprehension. New York: Academic Press.

VanLehn, K. (1989). Problem solving and cognitive skill acquisition. In: M. J. Posner (Ed.), Foundations of cognitive science. (pp. 527-579). Cambridge, MA: The MrT Press.

VanLehn, K. (1991). Rulle acquisition events in the discovery of problem-golving strate. gies. Cognitive Science, 15, 1-47.

Van Rossum, H. J. M., Bender, W., \& Meinders, A. E. (1991). De invloed van biografische details in casuistische mededelingen op het diagnostisch oordeel [The influence of biographical details in case reports on diagnostic judgment. Nederlands Tijdschrift voor de Geneeskunde, 135, 802-805.

Van Rossum, H. J. M., Brięt, E., Bender, W. \& Meinders, A. E. (1990). The transfer effect of one single patient demonstration on diagnostic judgment of medical students: Both better and worse. In: W. Bender, R. J. Hiemstra, A. J. J. A. Scherpbier, \& R. P. Zwierstra (Eds.), Teaching and assessing clinical competence. Groningen, The Netherlands: BoekWerk Publications.

Vosniadou, S., \& Brewer, W. F. (1987). Theories of knowledge restructuring in development. Review of Educational Research, 57,51-67.

Wagenaar, W. A. (1987). Logisch voorwaarts en intelligent achterwarts. Modellen voor het stellen van diagnoses in de hulpverlening. [Logically forward and intelligently back ward. Diagnostic models for psychollogical assistencel. In: J. D. wan der Ploeg, \& P. M. van den Bergh (Eds.), Besluitvorming en jeugdhulpuerlening. (pp. 9-18). Leuven/Amersfoort: Acco.

Waldrop, M. M. (1984). The necessity of knowledge. Science, 223, 1279-1282.

Walker, C. H., \& Yekowich, F. R. (1984). Script-based inferences: Effects of text and knowledge variables on recognition memory. Journal of Verbal Learning and Verbal Behavior, 23, 357-370.

Walker, C. H., \& Yekovich, F. R. (1987). Activation and use of script-based antecedents in anaphoric reference. Journal of Memory and Language, 26, 673691.

Weber, E. U., Böckenholt, U., Hilton, D. J., \& Wallace, B. (1993). Determinants of diag. nostic hypothesis generation: Effects of information, base rates, and experience. Journal of Experimental Psychology: Learning, Memory, and Cognition, 19, 11511164.

Wilkins, R. W. (1970). Internal Medicine. In: C. S. Keefer, \& R. D. Wilkins (Eds.), Medicine. Essentials of clinical practice. (pp. 1-5). Boston: Little, Brown and Company.

Wineburg, S. (1991). Historical problem solving: A study of the cognitive processes used in the evaluation of documentary and pictorial evidence. Journal of Educational Psychology, 83,73m8.

Wisniewsky, E. J. \& Medin, G. (1994). On the interaction of theory and data in concept learning. Cognitive Science, 18, 221-281.

Wohlwill, I. F. (1973). The study of behavioral development. New York: Academic Pregs.

Wolters, G. (1983). Episodic memory. Encoding distinctiveness and memory perfor. mance. Leiden, The Netherlands: University of Leiden, Academisch Proefschrift.

Woodworth, R. S. (1958). Dynamics of behavior. New York: Holt.

Wortman, P. (1966). Representation and strategy in diagnostic problem solving. IEEE Transactions on Human Factors in Electronics, 8, 48-53.

Wortman, P. M. (1970). Cognitive utilization of probabilistic cues. Behavioral Science, 15, $329-336$.

Yekovich, F. R., \& Walker, C. H. (1986). Retrieval of scripted concepts. Journal of Memory and Language, $25,627-644$.

Yekovich, F. R., \& Walker, C. H. (1987). The actiwation and use of scripted knowledge in reading about routine activities. In $B$. $K$. Britton \& S. M. Glynn (Eds.), Executive control processes in reading. Hilsdale, NJ: Lawrence Erlbaum Associates, Publishers. 



\section{APPENDIX 2.A}

\section{STATEMENT TEXTS AND PERCENTAGE OF SUBJECTS THAT SELECTED THESE STATEMENTS}

\begin{tabular}{|c|c|c|}
\hline Type & \% sell. ${ }^{b}$ & Statement Text \\
\hline Rest & 77.42 & Physical examination reveals: \\
\hline $\mathrm{EC}$ & 67.74 & Rutger, 17 years old, 5th grade HAVOc \\
\hline $\mathrm{EC}$ & 61.29 & Shows up during office hours with the complaint of: \\
\hline Con & 53.23 & Neither liver nor splteen are enlarged \\
\hline Con & 50.00 & "Lately, I feel so spiritless and languid" \\
\hline $\mathrm{EC}$ & 48.39 & Eldest sister (21) has had glandular fever three months ago \\
\hline Con & 46.77 & Bilateral enlargement of lymph glands in neck and arm-pits \\
\hline Con & 45.16 & A few days ago, he has had a severe throat-ache, but it has subsided \\
\hline EC & 43.56 & At the age of eight, tonsillectomy was performed \\
\hline $\mathrm{EC}$ & 43.55 & Middle child from a family of three children \\
\hline Con & 40.32 & However, he did not vomit \\
\hline Con & 38.71 & The preceding days, he has scarcely exten anything \\
\hline Con & 37.10 & He has also had a slight headache for a few days \\
\hline Con & 37.10 & Auscultation reveals no abnormalities \\
\hline Con & 35.48 & "At school, I am unable to keep my attention sustained" \\
\hline Con & 33.87 & "At home, I don"t do anything more then just lying on the couch" \\
\hline $\mathrm{EC}$ & 33.87 & Mother phones: "Can you please come and have a look at my son" \\
\hline Con & 33.87 & "Yesterday, I had a slight fever, 38 degrees Centigrade" \\
\hline Con & 30.65 & He has measured no fever \\
\hline Con & 29.03 & Examination of urine reveals no abnormalities \\
\hline Con & 29,08 & Examination reveals no rebound tenderness in the epigastrial region \\
\hline $\operatorname{Con}$ & 27.42 & "For already three days, I have a terrible stomach-ache" \\
\hline $\operatorname{Con}$ & 25.81 & "I have been troubled by diarrhoea for sme days" \\
\hline Con & 25.81 & He denies having eaten anything but the usual stuff, this week \\
\hline Con & 25.81 & Palpation reveals: the liver is enlarged \\
\hline Con & 25.81 & His blood pressure is $130 / 75$ \\
\hline Con & 24.19 & Yesterday, I had considerable fever: 39.5 degrees Centigrade \\
\hline Con & 22.58 & "I don't feell quite well, for already more than a week" \\
\hline Con & 22.58 & He has no diarrhoea \\
\hline
\end{tabular}




\section{APPENDIX 2.A (Continued)}

Type $^{\mathrm{a}} \%$ sel. $^{\mathrm{b}}$

Statement Text

Con 22.58 Auscultation reveals: striking intestinal noises

Can 20.97 Man, 45 years, staff member of a technical service group

Con 19.36 Examination reveals no pain upon pressure in the epigastrial region

Con 17.74 He has no itching

Con $\quad 17.74$ Palpation shows no enlarged spleen

EC 17.74 A month ago, his brother (14 year) was diagnosed as having Hepatitis A

EC 16.13 Father of two children

EC 16.13 A week ago, his brother (14 years) has stayed in bed for six days with the flu

Con $\quad 12.90$ His blood pressure is $170 / 110$

Con 11.29 His blood pressure is 110/60

EC $\quad \mathbb{1 1 . 2 9}$ Known with chronic alcohol abuse

EC 9.67 At his work, many employees have got the flu

Con 9.67 The patient is in bed, and perspires heavily

Con $\quad 9.67$ Palpation reveals no enlarged liver

$\mathrm{EC} \quad 8.07$ Family history of diabetes mellitus

Con 6.45 "At work, I have difficulty to sustain my attention"

EC 4.84 A month ago, his youngest son (12 year) was diagnosed as having Hepatitis A

EC 3.23 Man, 71 years, retired since 8 years

$\mathrm{EC} \quad 3.23$ His wife calls: "Would you please come and have a look at my husband"

a Statement type, $\mathrm{EC}=$ Enabling Condition, Con = Consequence

b Percentage of subjects that selected this statement $(\mathrm{N}=62)$ 


\section{APPENDIX 3.A}

\section{EXAMPLES OF PROTOCOLS (target disease: "herpes zoster")}

\section{Enabling Conditions centered:}

eh, also an elderly man or woman, an elderly man or woman, also in a somewhat-what bad shape, a bit emaciated, perhaps; probably, but not necessarily, known with underlying affictions, - eh- who gets a rash, and pain, in a certain segment...

\section{Fault centered:}

it's the chicken-pox virus, you've had chicken-pox in your youth, and the virus settles down in your nerves, -and you get vesicles on your skin, and these are very contagious, because the fluid contains the virus, and you'll never get rid of it-; and it can cause an infection of the eyes, and that might be very dangerous...

\section{Consequences centered:}

-pain, unilateral, in the shape of a belt, and it coincides with a skin nerve, and eh- not always in the shape of a bell-, it might be, but not when it's on your head, or on your legs-; anyway, it coincides with a skin nerve, -redness, a swelling, an elevated swelling, with vesicles, and eh- hypersensitivity, and often a general feeling of weak health- a classical picture-

\section{CDFT centered:}

in the elderly often a problem, because they visit the physician in a stage when it's already too late to treat it properly-nowadays with Zovirax-pills; eh-very nasty disease, many problems with so-called post-herpetical pains, these may continue to trouble the patient for years and are very difficult to combat; eh- in the beginning stages often difficult to diagnose, -in case of vague, unexplainable pains I'm always on guard and ask them to return the next day 


\section{APPENDIX 3.B}

\section{RESULTS OF THE PATTENT FREQUENCY INVENTORY}

\begin{tabular}{|c|c|c|c|c|c|c|}
\hline \multirow[b]{2}{*}{ Disease } & \multicolumn{4}{|c|}{ Mean number of patients seen by } & \multicolumn{2}{|c|}{$\begin{array}{l}\mathrm{N}^{\mathrm{i}} \text { of fam. phys. } \\
\text { who indicated }\end{array}$} \\
\hline & 6th. yr. & Interns & $\begin{array}{l}\text { Fam. } \\
\text { Phys. }\end{array}$ & $\mathrm{A} 11$ & $\begin{array}{l}\text { Never } \\
\text { seen }\end{array}$ & $\begin{array}{l}20^{+} \\
\text {times }\end{array}$ \\
\hline $\begin{array}{l}\text { Meningitis as complication } \\
\text { of mumps }\end{array}$ & 0 & 0 & 2.32 & .82 & 11 & 1 \\
\hline Epidural hematoma & 1.52 & .78 & 1.57 & 1.28 & 6 & 0 \\
\hline Rupture of aortic aneurysm & 1.77 & 2.85 & 4.34 & 2.99 & 5 & 0 \\
\hline Pancreatic cancer & 1.96 & 2.63 & 6.52 & 3.69 & 2 & 1 \\
\hline Digitalis intoxication & 1.18 & 3.04 & 7.16 & 3.78 & 3 & 2 \\
\hline Urosepsis & 2.91 & 2.09 & 6.61 & 3.84 & 2 & 2 \\
\hline Pediculosis pubis & .23 & 3.30 & 11.59 & 5.02 & 0 & 4 \\
\hline Hepatitis A & .98 & 2.04 & 13.05 & 5.31 & 0 & 7 \\
\hline Dermatitis peri-oralis & 2.71 & 6.07 & 14.50 & 7.73 & 1 & 11 \\
\hline Metastases in lungs & 7.27 & 7.83 & 13.23 & 9.42 & 0 & 10 \\
\hline Monilia of the mouth & 2.59 & 8.15 & 18.05 & 9.58 & D & 17 \\
\hline Stomatitis aftosa & 2.43 & 11.93 & 18.73 & 11.03 & 0 & 18 \\
\hline Herpes zoster & 5.00 & 9.96 & 18.50 & 11.20 & 0 & 15 \\
\hline Kidney stones colic & 2.09 & 13.17 & 18.77 & 11.37 & 0 & 16 \\
\hline Perforated otitis media & 2.41 & 12.63 & 19.50 & 11.53 & 0 & 19 \\
\hline Pre-infarct syndrome & 6.41 & 10.50 & 17.75 & 11.54 & 0 & 16 \\
\hline Dyspepsia on a nervous basis & 6.18 & 16.39 & 19.27 & 13.99 & 0 & 19 \\
\hline Vaginal candidiosis & 6.32 & 18.57 & $20+$ & 15.02 & 0 & 22 \\
\hline Nervous abodminal pain & 10.09 & 18.70 & $20+$ & 16.30 & 0 & 22 \\
\hline
\end{tabular}




\section{APPENDIX 4.A}

\section{NAMES OF THE DISEASES USED IN THE STUDY}

Diseases on which the three practice cases were based

Perforated otitis media

Monilia of the mouth

Dyspepsia of a nervous origin (also called: nervous gastritis)

\section{Diseases on which the 16 experimental cases were based}

Metastatic sigmoid cancer (metastases in the lungs)

Aneurysm of the aortic artery (threatening rupture)

Urosepsis

Dermatitis peri-oralis

Vaginal candidiosis

Kidney stones (colic)

Carcinoma of the head of the pancreas

Stomatitis aftosa (multiple small ulcers in the mouth)

Digitalis intoxication

Epidural hematoma

Nervous abdominal pain

Pediculosis pubis

Herpes zoster

Meningitis or encephalitis as a complication of mumps

Hepatitis A

Pre-infarct syndrome

Diseases on which the five "NO-cases" were based

Pfeiffer's disease (announced as "Is this a patient with Hodgkin"s disease")

Pernicious anemia (announced as: "Is this a patient with iron-deficiency anemia or hypochromatic anemia")

Perforated gastric ulcer (announced as: "Is this a patient with refluxoesophagitis")

Seborrheic dermatitis (announced as: "Is this a patient with impetigo")

Thyrotoxicosis (announced as: "Is this a patient with hypo-thyroid") 


\section{APPENDIX 4.B}

\section{EXAMPLE OF A CASE DESCRIPTION WTTH FOUR CASE VARIANTS}

\section{Case 6: kidney stones (colic)}

The number preceding each text phrase refer to the number of the screen image in which that particular phrase was presented

\section{PP-variant}

1. Man, age 47, married, three children

2. Occupation: store-keeper

3. Medical history: bronchitis at age 30

4. Had his leg broken six years ago, as a consequence of a car accident

5. Four years ago: treated with medicaments for kidney stones

6. Some of his relatives are known to have coronary disease and diabetes mellitus

7. His wife rings up, asks the physician for an immediate visit:

8. Just like a few years ago, her husband is rolling across the room because of the pain. He is also vomiting almost continuously

9. When the physician arrives, the pain thas just subsided. The patient is sitting on the sofa and recovering a bit

10. At the moment, the patient doesn't look very ill

11. He complains about having had a convulsive abdominal pain abreast of the navel, at the left side

12. The pain is radiating to his groins

13. The pain emerges very suddenly, and then gradually subsides. During an attack he almost can't stand it

14. Earlier that day he had already seen some blood in his urine, but had no pain at the time

15. Is this a patient with a kidney stone colic? (yes/no)

16. Please estimate the probability that this person has a kidney stone colic in a percentage (range between $0 \%-100 \%$ )

\section{Comments:}

The prototypical patient with kidney stone colic is a male, aged 35 to 55 years (state ment 1), who has had this kind of complaint before (statement 5 ). As the clinical picture often is rather impressive, it is not unusual that relatives are in panic (statement 7 ). Typically, the pain is of a convulsive nature, about half-way down the abdomen, and one-sided (left or right, statement 11). Radiation to the groins is also very prototypical (statement 12). The same holds for the pain-free intervals, during which the patient appears normal (statement 9, 10, 13). During an attack, people are literally runining or cringing (statement 8,13 ). Blood in the urine may also occur, before or after an attack (statement 14). The statements $2,3,4$ and 6 are added to complete the case-description (occupation is usually mentioned, as are some rather irrelevant medical history items with regard to the present condition) 


\section{APPENDIX 4.B (Continued)}

\section{AP-variant}

1. Woman, 32 years, married, two children

2. Occupation: works at the clerical staff of a large school

3. Medical history: bronchitis at age 20

4. Had her leg broken six years ago, as a consequence of a car accident

5. Has been taking the contraceptive pill for nime years, interrupted for pregnancies

6. Some of her relatives are known to have coronary disease and diabetes mellitus

7. Her husband rings up, asks the physician for an immediate visit:

8. He is afraid that his wife is dying: she is rolling through the room because of the pain. She is also vomiting almost continuously

9. When the physician arrives, the pain has just subsided. The patient is sitting on the sofa and recovering a bit

10. At the moment, the patient doesn't look very ill

11. She complains about having had a convulsive abdominal pain abreast of the navel, at the left side

12. The pain is radiating to her groins

13. The pain emerges very suddenly, and then gradually subsides. During an attack she almost can't stand it

14. Earlier that day she had already seen some bload in her urine, but had no pain at the time

15. Is this a patient with a kidney stone colic? (yes/no)

16. Please estimate the probability that this person has a kidney stone colic in a percentage (range between 0\%-100\%)

\section{Comments:}

Kidney stones are not often found in young women. In addition, nothing in her medical history points to the possible existence of this ailment. 'The symptoms (the same as in the previous description) are very prototypical, though.

\section{PA-variant}

1. Man, age 47, married, three children

2. Occupation; store-keeper

3. Medical history: bronchitis at age 30

4. Had his leg broken six years ago, as a consequence of a car accident

5. Four years ago: treated with medicaments for kidney stones

6. Some of his relatives are known to have coronary disease and diabetes mellitus

7. The patient appears at consulting hour with the complaint:

8. Since the other day, occasionally a sharp, stabbing pain in the abdomen, threast of the navel, at the right side

9. The pain is radiating to his groins

10. He had already felt for few days a somewhat nagging sensation in this part of his abdomen

11. And it is still nagging, now, in between the stabs

12. Upon inquiry, the patient admits having felt an urge to micturate more often than usual, the last few days, but the amount of urine each time was small

13. He says he doesn't feel very well

14. And reports having measured 37.8 degrees (Centigrade) temperature

15. Is this a patient with a kidney stone colic? (yes/no)

16. Please estimate the probability that this person has a kidney stone colic in a percentage (range between $0 \%-100 \%$ ) 


\section{APPENDIX 4.B (Continued)}

\section{Comments (PA variant):}

This pattern of symptoms and complaints is possibly, but not very likely caused by kidney stones.

\section{AA-variant}

1. Woman, 32 years, married, two children

2. Occupation: works at the clerical staff of a large school

3. Medical history: bronchitis at age 20

4. Had her leg broken six years ago, as a consequence of a car accident

5. Has been taking the contraceptive pill for nine years, interrupted for pregnancies

6. Some of her relatives are known to have coronary disease and diabetes mellitus

7. The patient appears at consulting hour with the complaint:

8. Since the other day, occasionally a sharp, stabbing pain in the abdomen, abreast of the navel, at the right side

9. The pain is radiating to her groins

10. She had already felt for a few days a somowhat nagging sensation in this part of her abdomen

11. And it is still nagging, now, in between the stabs

12. Upon inquiry, the patient admits having felt an urge to micturate more often than usual, the last few days, but the amount of urine each time was small

13. She says she doesn't feel very well

14. And reports having measured 37.8 degrees (Centigrade) temperature

15. Is this a patient with a kidney stone colic? (yes/no)

16. Please estimate the probability that this person has a kidney stone colic in a percentage (range between $0 \%-100 \%$ )

\section{Comments:}

Upon seeing this patient and hearing her complaints, the physician will probably not immediately think of kidney stones. However, the possibility of this disease is surely not ruled out completely. 


\section{APPENDIX 4.C}

\section{NUMBER OF STATEMENTS (SCREEN IMAGES) AND WORDS PER CASE DESCRIPTION}

Practice cases

Perforated otitis media

Monilia of the mouth

Nervous gastritis

\section{Experimental cases}

Metastatic sigmoid cancer

Rupturing aneurysm

Urosepsis

Dermatitis peri-oralis

Vaginal candidiosis

Kidney stones colic

Carcinoma of the head of the pancreas

Stomatitis aftosa

Digitalis intoxication

Epidural hematoma

Nervous abdominal pain

Pediculosis pubis

Herpes zoster

Meningitis (as a complication of mumps)

Hepatitis A

Pre-infarct syndrome

NO cases

Hypo-thyroid

Impetigo

Iron-deficiency anemia*

Hodgkin"s disease

Reflux oesophagitis

* this case was omitted from the analysis

number of statements number of words

10

98

9

81

17

177

Number of statements Number of words per case variant

PP AP PA AA

$\begin{array}{lllll}14 & 137 & 128 & 137 & 138\end{array}$

14. $172 \quad 176 \quad 164 \quad 167$

$14 \quad 132 \quad 131 \quad 132 \quad 131$

$14 \quad 172 \quad 176 \quad 164 \quad 167$

$14 \quad 136 \quad 166 \quad 144 \quad 164$

$14 \quad 167 \quad 165 \quad 138 \quad 147$

$\begin{array}{lllll}15 & 150 & 145 & 159 & 155\end{array}$

$\begin{array}{lllll}10 & 94 & 94 & 85 & 56\end{array}$

$\begin{array}{lllll}12 & 110 & 130 & 123 & 117\end{array}$

$\begin{array}{lllll}14 & 180 & 160 & 178 & 157\end{array}$

$\begin{array}{lllll}19 & 193 & 216 & 201 & 236\end{array}$

$\begin{array}{lllll}8 & 90 & 106 & 93 & 109\end{array}$

11 $\quad 105 \quad 94 \quad 106 \quad 96$

$\begin{array}{lllll}13 & 121 & 122 & 115 & 117\end{array}$

$\begin{array}{lllll}17 & 140 & 140 & 147 & 140\end{array}$

$\begin{array}{lllll}18 & 160 & 178 & 185 & 186\end{array}$

number of statements

number of words

number of words 


\section{APPENDIX 4.D}

\section{FXPERIMENTAL DESIGN OF THE SEQUENCES}

\begin{tabular}{|c|c|c|c|c|c|c|c|}
\hline $1 \mathrm{~A}$ & $2 \mathrm{~A}$ & $3 \mathbf{A}$ & $4 \mathrm{~A}$ & 18 & 28 & $\mathfrak{B}$ & 48 \\
\hline pr1 & prl & pr1 & pr1 & prl & pr1 & pr1 & pri 1 \\
\hline $\mathrm{pr} 2$ & pr2 & $\mathrm{pr}^{2}$ & pr2 & prz & $\mathrm{pr} 2$ & pr2 & pr2 \\
\hline prs & pr3 & pr3 & pr3 & pr3 & pr3 & pr3 & pr3 \\
\hline 4 & 4 & 4 & 4 & $14 A P$ & $14 \mathrm{PA}$ & $14 \mathrm{AA}$ & 14PP \\
\hline $5 \mathrm{PA}$ & $5 \mathrm{AA}$ & $5 \mathrm{PP}$ & $5 \mathrm{AP}$ & $15 \mathrm{PP}$ & $15 \mathrm{AP}$ & $15 \mathrm{PA}$ & 15AA \\
\hline $6 \mathrm{PA}$ & 6AA & $6 \mathrm{PP}$ & $6 \mathrm{AA}$ & 16 & 16 & 16 & 16 \\
\hline 7AA & $7 \mathrm{PP}$ & $7 A P$ & $7 \mathrm{PA}$ & $17 \mathrm{AA}$ & $17 \mathrm{PP}$ & $17 \mathrm{AP}$ & $17 \mathrm{PA}$ \\
\hline 8 & 8 & 8 & 8 & $18 \mathrm{PA}$ & $18 \mathrm{AA}$ & $18 \mathrm{PP}$ & 18AP \\
\hline 9AA & 9PP & $9 \mathrm{AP}$ & 9PA & $19 P A$ & $19 A$ & 19PP & 19AP \\
\hline $10 \mathrm{PP}$ & $10 \mathrm{AP}$ & 10PA & 10AA & 20 & 20 & 20 & 20 \\
\hline $11 \mathrm{AP}$ & IIPA & $11 \mathrm{AA}$ & $11 P P$ & $21 P P$ & $21 \mathrm{AP}$ & 21PA & $21 \mathrm{AA}$ \\
\hline $12 \mathrm{AA}$ & $12 \mathrm{PP}$ & $12 A P$ & $12 \mathrm{PA}$ & 22 & 22 & 22 & 22 \\
\hline $13 \mathrm{AP}$ & $13 \mathrm{PA}$ & $13 \mathrm{AA}$ & $13 \mathrm{PP}$ & $23 \mathrm{PP}$ & 23AP & $23 \mathrm{PA}$ & $23 \mathrm{AA}$ \\
\hline & & & & $24 \mathrm{AP}$ & 24PA & $24 \mathrm{AA}$ & $24 \mathrm{PP}$ \\
\hline $14 \mathrm{AP}$ & $14 \mathrm{PA}$ & $14 \mathrm{AA}$ & $14 \mathrm{PP}$ & & & & \\
\hline $15 \mathrm{PP}$ & $15 \mathrm{AP}^{\prime}$ & $15 \mathrm{PA}$ & $15 \mathrm{AA}$ & 4 & 4 & 4 & 4 \\
\hline 16 & 16 & 16 & 16 & $5 \mathrm{PA}$ & $5 \mathrm{AA}$ & $5 P P$ & $5 A P$ \\
\hline $17 \mathrm{AA}$ & $17 \mathrm{PP}$ & $17 \mathrm{AP}$ & $17 \mathrm{PA}$ & $6 \mathrm{PA}$ & $6 \mathrm{AA}$ & 6PP & $6 \mathrm{AA}$ \\
\hline $18 P A$ & $\mathbb{1 8 A A}$ & 18PP & $18 \mathrm{AP}$ & $7 \mathrm{AA}$ & $7 \mathrm{PP}$ & TAP & $7 \mathrm{PA}$ \\
\hline $\mathbb{1 9 P A}$ & $19 \mathrm{AA}$ & $19 \mathrm{PP}$ & $19 \mathrm{AP}$ & 8 & 8 & 8 & 8 \\
\hline 20 & 20 & 20 & 20 & 9AA & $9 P P$ & 9AP & 9PA \\
\hline $2 \mathbb{I P P}$ & $21 \mathrm{AP}$ & $21 \mathrm{PA}$ & 21AA & $10 \mathrm{PP}$ & $10 \mathrm{AP}$ & $10 \mathrm{PA}$ & 10AA \\
\hline 22 & 22 & $\mathscr{2}$ & 22 & 11AP & $11 \mathrm{PA}$ & 11AA & $\mathbb{1} \mathbb{1 P P}$ \\
\hline $23 \mathrm{PP}$ & $23 \mathrm{AP}$ & 23PA & $23 \mathrm{AA}$ & $12 \mathrm{AA}$ & $12 \mathrm{PP}$ & $12 \mathrm{AP}$ & $12 \mathrm{PA}$ \\
\hline $24 \mathrm{AP}$ & $24 \mathrm{PA}$ & $24 \mathrm{AA}$ & $24 \mathrm{PP}$ & $13 \mathrm{AP}$ & $13 P A$ & $13 A A$ & $13 \mathrm{PP}$ \\
\hline
\end{tabular}

\section{Note}

Each sequence starts with three practice cases, which are the same for all sequences. The experimental cases fall into one of four groups, with the same case variant for each member of a particular group in a given sequence. For example, cases $10,15,21$, and 23 belong to one group, and in sequence 1 the PP variant of these cases is presented, un sequence 2 the AP variant, in sequence 3 the PA variant and in sequence 4 the AA variant. Each sequence contains four cases of each case variant.

Cases $4,8,16,20$ and 22 are examples of $\mathrm{NO}$-cases, of which only one variant exists. The $A$ - and B-forms of each sequence differ only in the order in which the experimental cases are presented. 


\title{
APPENDIX 5.A
}

\section{EXAMPLE OF A CASE DESCRIPTION AND THE ASSOCIATED TEST STATEMFNTS}

\author{
case: kidney stones colic
}

1. Man, aged 47

2. He is married and has three teenage children

3 . His occupation is store-keeper

4. At age 30, he was treated for bronchitis

5. Six years ago, he had his leg broken as a consequence of a car accident

6. Four years ago, he was treated with medicaments for kidney stones

7. Some of his relatives are known with coronary diseases and diabetes mellitus

8. His wife rings up, asks the physician for an immediate visit: it's happening again

9. Her husband is vomiting almost continuously

10. He is rolling across the room because of the pain

11. At the moment the physician arrives, the pain has just subsided

12. The patient is sitting on the sofa, recovering a little

13. He complains about having had a convulsive abdominal pain at the left side, abreast of the navel

14. The pain extends to his groins

15. The pain emerged all of a sudden, and subsequently gradually subsided

16. During an attack, he almost can't stand it

17. Earlier that day, he had already seen some blood in his urine

18. But he had no pain at that time

19. His wife says she has measured his temperature: $38.2^{\circ}$ Centigrade 


\section{APPENDIX 5.B}

\section{TESTITEMS FOR THE KIDNEY STONES COLIC CASE DESCRIBED IN APPENDIX 5.A}

(The actual order of the items in the test was randomly determined)

Typ $^{1}$ Pres $^{2}$ Script $^{3}$

Item text

$\begin{array}{llll}P & \mathrm{~S} & \mathrm{EC} & \mathrm{Man}, \text { aged } 47\end{array}$

$\mathrm{P} \quad \mathrm{S}$ Con1 He is rolling across the room because of the pain

$\mathrm{P} \quad \mathrm{S}$ Con2 The patient is sitting on the sofa, recovering a bit

P U EC Four years ago, he had a kidney stone colic

P U Con $1^{4}$ The pain radiated

P U Con25 In-between the attacks, he doesn't look very ill

A S EC Six years ago, he had his leg broken as a consequence of a car accident

A $S$ Con Earlier that day, he had already seen some blood in his urine

A U EC He is slightly overweight

A U Con He has a mild fever

I U EC The patient appears at the consulting hour

I. U Con The pain gradually increases in severity, but then suddenly disappears

$1 \mathrm{~T} y \mathrm{p}=$ item typicality ( $\mathrm{P}=$ prototypical, $\mathrm{A}=$ atypical, $\mathrm{I}=$ inconsistent)

2 Pres= textual presence ( $S=$ stated, $U=$ unstated)

${ }^{3}$ Script $=$ script component ( $\mathrm{EC}=$ Enabling Condition, $\mathrm{Con}=\mathrm{Consequence)}$

4 paraphrase (cf. Appendix A, statement 14)

5 omission (is typical for kidney stones colic, but not mentioned in the case described in Appendix A) 


\section{ACKNOWLEDGEMENTS}

This thesis is an issue in a series of theses on the topic of medical expertise development, some of which have already been published, while others are forthcoming. All these dissertations were or are being prepared at the Department of Educational Research and Development (O\&O) of the University of Limburg at Maastricht, the Netherlands. The "founding father" of these projects, Henk G. Schmidt, was also my promotor; he is the first one I want to thank for his contribution. As can be read from the list of references included in this thesis, in the the field of medical expertise development, the name of Henk G. Schmidt is almost inseparably connected to that of Henny P. A. (Els) Boshuizen. She was my co-promotor and receives full credits for keeping me on the right track. Both Henk and Els were directly involved in the process of preparing and performing the experiments, and writing the thesis. Especially Els was almost always there to help me solve both the smaller and the more difficult problems.

Next, I want to thank my direct colleagues and members of the "cognitive psychology reading club:" Herco Fonteijn, Maureen Machiels-Bongaerts, Margje van de Wiel, and Rina Vaatstra. Our arguments and discussions concerning the flaws of experiments and articles in (often scientifically outstanding) journals have given me the courage to continue and the impression that, regardless of many shortcomings, the experiments I designed and performed were, after all, worthwile.

In addition, $I$ want to thank the Department of Educational Research and Development for providing the facilities to do the research and write my thesis. Especially the opportunity to visit the Annual Meeting of the American Educational Research Association in 1992 (San Francisco), 1993 (Atlanta), and 1994 (New Orleans) was very valuable; without financial support of the "vakgroep," this would have been impossible. For visiting the AERA, I am also grateful to the Dutch Foundation for Scientific Research (NWO), who also sponsored my voyages to America. Three names will, in my memory, be always attached to the acronym AERA: Those of Paul Feltovich, Geoffrey Norman, and Vimla Patel. Though living and working thousands of miles away, for one week each year, we were colleagues.

Furthermore, I appreciate the contributions of Harry Crebolder, Pie Hobus, Karel Jansen, Saskia Mol, Jan wan Ree, Lambert Schuwirth, Hetty Balendong, and Paul Zwietering: They judged the plausibility of the medical materials I wast unfamiliar with, corrected the case descriptions I had constructed, and/or volunteered to be pilot subjects in my experiments. Bart van Swol and Annie Bosman from the University of Amsterdam were so kind as to let me use the experimental program PET, a device for measuring reaction times. Gerard van Breukelen advised me about some statistical issues. Also Bart Reuten has to be thanked heartfully for the effort he put into programming the experimental tasks. Bert Kerkhofs helped me often when I had troubles with my computer or with Maasnet.

Finally, I want thank my parents and other relatives who have supported me during these years. Though their contribution to the content of this thesis is probably limited, in many other ways they gave me full support. Especially my brother deserves special thanks for giving me the final impetus to finish this thesis and take my degree just one week before he will take his.

Last but not least, I want to thank the 45 (then) fourth-year students, 72 (then) sixth-year students, 23 (then) interns, and 75 (most of them probably still) familly physicians, who participated in the studies. Most of them enjoyed being 
experimental subjects, though some of them found the tasks they were subjected to a little bit odd. Nevertheless, regardless how valuable the contributions of the persons and institutions mentioned in the previous sections have been, without the contribution of students and family physicians, it would have been entirely impossible to write this thesis.

\section{Eugène Custers}

March 1995 


\section{CURRICULUM VITAE}

Eugène Custers was born on June 30,1961 in Swalmen. He finished gymnasium-B at the Bisschopelijk College "Broekhin", Roermond, in 1979. He studied psychology at the Free University Amsterdam (VU), where he graduated in cognitive psychology (June 1988) and developmental psychology (August 1988). From June 1988 till December 1989 he participated as an assistant researcher in a project on childrens' attention in the classroom at the Catholic University Brabant (KUB) as substitute for military service. In January 1990 he became Assistent Researcher (AIO) at the Maastricht Faculty of Medicine, Department of Educational Research and Development. Here, he participated in the research project "Development of medical expertise." 